\author{
Universidade de SÃo PaUlo \\ Instituto de Física de SÃo Carlos \\ Departamento De Física e Informática
}

\title{
Espectro de energia-momento para a Cromodinâmica Quântica na Rede
}

\author{
Antônio Francisco Neto
}

Tese apresentada ao Instituto de Física de São Carlos, Universidade de São Paulo, para obtenção do Título de Doutor em Ciências: Fisica Básica

Orientador: Prof. Dr. Paulo Afonso Faria da Veiga

SÃo CARlos 


\section{Neto, Antônio Francisco}

Espectro de energia-momento para a Cromodinâmica Quântica na Rede /Antônio Francisco Neto.

- São Carlos, 2005

$115 \mathrm{p}$.

Tese (Doutorado) - Instituto de Física de São Carlos, 2005.

Orientador: Prof. Dr. Paulo Afonso Faria da Veiga

1. Espectro de energia-momento 2. Cromodinâmica Quântica 3. Equação de Bethe-Salpeter I. Título. 
Aos meus pais 


\section{Agradecimentos}

Este trabalho foi financiado pelo Conselho Nacional de Desenvolvimento Científico e Tecnológico (CNPq)

Gostaria de agradecer a Deus, aos meus queridos pais Wilson de Andrade Francisco e Lêda Fortes Francisco, por todo amor, por me ensinarem o caminho certo a seguir do ponto de vista ético, o valor do trabalho e a ter persistência. Eu tenho um profundo orgulho de ser filho de vocês. À minha querida irmã Amanda Fortes Francisco, meus avós Antônio Fortes e Carlota de Castro Fortes. À minha querida esposa Leida Armond Muzzi, pelo amor, pela companhia e carinho ao longo destes 4 anos de convívio e 1 de casamento.

Aos meus dois orientadores: Paulo Afonso Faria da Veiga e Michael Louis O'Carroll. Não só pela oportunidade, mas por todo conhecimento compartilhado, pela disponibilidade, generosidade, enfim, por me proporcionarem tudo aquilo que eu esperava de uma orientação. Sinceramente, a minha admiração profissional só aumentou ao longo destes 4 anos de convívio. Além de tudo que aprendi, hoje em dia tomo mais cuidado ao dizer que cheguei (ou chegamos) à versão final de algum trabalho.

Aos meus colegas que de uma forma ou outra me ajudaram, seja com uma palavra amiga, com o Maple, com o Latex, etc. Na UFMG, Adélcio Carlos de Oliveira, Ângelo Malachias de Souza, Leonardo Teixeira Neves, Gisele Nogueira Fontes, Guilherme José de Moraes Garcia, Gustavo Aquino Lima e Rafael Santos Thebaldi. Na USP São Carlos, Alexandre Colato, Antonio Carlos Ferreira Seridonio, Leonardo Paulo Maia, Paulo Alexandre de Castro, Petrus Henrique Felício Cândido Leão Ribeiro dos Anjos (nome de batismo), Vivaldo Leiria Campo Jr.

Ao CNPq por esses sete anos de bolsa cujo início data de 1998 quando me iniciei em atividades de pesquisa através de uma bolsa de Iniciação Científica (PIBIC) na UFOP. Ao Instituto de Física da USP-São Carlos pela oportunidade ao me conceder a bolsa de doutorado e pela hospitalidade. Em especial agradeço ao grupo de física teórica que me acolheu e ao Prof. Lidério Ioriatti Jr. 


\section{Resumo}

Neste trabalho, consideramos a obtenção do espectro de energia-momento para modelos de Cromodinâmica Quântica (QCD) na rede, na formulação integral funcional, com tempo imaginário e no regime de acoplamento forte. Este regime corresponde a tomar o parâmetro de "hopping" $\kappa>0$ satisfazendo $1>>>>\beta>0$, onde $\beta \equiv g_{0}^{-2}$ é o parâmetro de acoplamento da interação dos campos de calibre entre si, aqui definidos na álgebra de Lie $S U(3)$. Consideramos modelos de QCD com campos fermiônicos de quarks com um ou dois sabores (isospin). Para os casos de $2+1$ e $3+1$ dimensões, um sabor, e usando matrizes de spin de Dirac $4 \times 4$, mostramos a existência de mésons, como estados ligados de um quark e um anti-quark, a qual é detectada pelo aparecimento de curvas de dispersão isoladas no espectro de energia-momento no subespaço de Hilbert físico associado a um número par de férmions. As massas dos mésons são assintoticamente dadas por $-2 \ln \kappa$. Resultados similares foram também obtidos para o modelo em dimensão $2+1$, um e dois sabores, e matrizes de spin de Pauli $2 \times 2$. Usando resultados anteriores sobre a existência de bárions no subespaço com um número ímpar de quarks, consideramos a questão da existência de estados ligados de dois hádrons: dois mésons, e um bárion e um méson, nos modelos em dimensão $2+1$, com um e dois sabores, e matrizes de spin de Pauli $2 \times 2$. Nossa análise é fortemente baseada na obtenção de propriedades analíticas de decaimento em distância para as funções de correlação para duas e quatro partículas, obtidas usando uma adaptação do método de desacoplamento de hiperplanos. A análise de estados ligados com duas partículas é realizada a partir de uma equação de Bethe-Salpeter para a rede numa aproximação escada (ordem dominante em $\kappa$ ). Mostramos que estados ligados de dois mésons ou um méson e um bárion não existem para o modelo com um único sabor, onde os efeitos da repulsão de Pauli excluem a existência de atração entre as partículas. Para o caso de dois sabores, restringindo nossa análise ao setor de isospin total nulo, um estado ligado do tipo méson-méson foi detectado, com energia de ligação dada aproximadamente por $0,02359 \kappa^{2}$. O potencial atrativo entre as duas partículas, neste caso, advém de dois tipos de contribuições: troca de (quasi-)mésons (um quark e um antiquark) e de um potencial dependente da energia total do sistema. Nós mostramos também que, a fonte principal de atração é um efeito de correlações de calibre. Usando técnicas já desenvolvidas para tratar este tipo de sistema físico, nossos resultados espectrais poderão ser estendidos para uma análise rigorosa, i.e. uma análise do espectro além da aproximação escada, para o modelo completo. 


\section{Abstract}

In this work, we consider the energy-momentum spectrum of lattice quantum chromodynamics (QCD) models, using the functional integral formulation, with imaginary time and in the strong coupling regime. This regime corresponds to taking the hopping parameter $\kappa>0$ satisfying $1>>\wedge>\beta>0$, where $\beta \equiv g_{0}^{-2}$ is the coupling parameter among the gauge fields, here defined in the $S U(3)$ Lie algebra. We consider QCD models with fermionic quark fields with one and two flavors (isospin). For the cases $2+1$ and $3+1$ dimensions, one flavor, and using $4 \times 4$ Dirac matrices, we show the existence of mesons, as bound states of one quark and one anti-quark, which is detected by the appearance of an isolated dispersion curve in the energy-momentum spectrum in the physical Hilbert space associated with an even number of fermions. The meson masses are asymptotically given by $-2 \ln \kappa$. Similar results were also obtained for the model in $2+1$ dimensions, with one and two flavors, and using Pauli matrices $2 \times 2$. Using results previously established about the existence of baryons in the subspace with an odd number of quarks, we consider the question of the existence of bound states of two hadrons: two mesons, and one baryon and one meson, in models with dimension $2+1$, with one and two flavors, and Pauli matrices $2 \times 2$. Our analysis is strongly based in analytic properties of decay for correlation functions for two and four particles, obtained using an adaptation of the decoupling of hyperplane method. The analysis of bound states of two particles is made using a Bethe-Salpeter equation on the lattice in a ladder approximation (leading order in $\kappa$ ). We show that bound states of two mesons or one meson and one baryon do not exist in the one flavor case. Here, Pauli repulsion overcomes any kind of attraction between the particles. For the two flavor case, restricting our analysis to the total isospin zero sector, a bound state of the type meson-meson was detected, with binding energy given approximately by $0.02359 \kappa^{2}$. The attractive potential between the two mesons comes from two kinds of contributions: exchange of a (quasi-)meson (a quark and an anti-quark) and an energy-dependent potential. We also show that, the main source of attraction is a gauge correlation effect. Using technics already developed to treat this kind of physical system, our spectral results can be extended to a rigorous analysis, i.e. a spectral analysis beyond the ladder approximation for the full model. 


\section{Sumário}

1 Introdução 1

2 Existência de mésons em $(2+1)$ e $(3+1)$ dimensões, um sabor e matrizes $\begin{array}{ll}\text { de Dirac } 4 \times 4 & 9\end{array}$

2.1 Apresentação dos resultados sobre a existência de um méson e um bárion . . . 10

2.2 Cotas para o decaimento e comportamento a pequenas distâncias da função de dois mésons $G$ e sua inversa por convolução $\Gamma \ldots \ldots$. . . . . . . . . . 22

2.3 Resultados Espectrais para um méson . . . . . . . . . . . . . . . 33

3 Ausência de estados ligados de méson-méson e méson-bárion em $(2+1)$ dimensões, um sabor e matrizes de Pauli $2 \times 2 \quad 40$

3.1 A função parcialmente truncada de quatro pontos para mésons e méson-bárion 41

3.2 A equação de Bethe-Salpeter na rede para o caso méson-méson . . . . . . . . . 45

3.3 Ausência de estados ligados do tipo méson-méson, em $2+1$ dimensões, um sabor e matrizes de spin $2 \times 2 \ldots \ldots \ldots \ldots$. . . . . . . . . . . . 49

3.4 Ausência de estados ligados do tipo méson-bárion, em $2+1$ dimensões, um sabor e matrizes de $\operatorname{spin} 2 \times 2 \ldots \ldots \ldots \ldots \ldots$

4 Um estado ligado méson-méson em $(2+1)$ dimensões, dois sabores e matrizes de Pauli $2 \times 2$

4.1 A função de dois pontos e quatro pontos para mésons em $2+1$ dimensões, dois sabores e matrizes de $\operatorname{spin} 2 \times 2 \ldots \ldots \ldots \ldots$

4.2 Simetria de isospin U(2) global e funções de correlação . . . . . . . . . . . . 67

4.3 A aproximação escada para a equação de Bethe-Salpeter: caso méson-méson, dois sabores . . . . . . . . . . . . . . . . . . . 69

4.4 Solução da equação de Bethe-Salpeter na aproximação escada para K . . . 76 
A Fórmula de Creutz para o Cálculo de Integrais de Calibre $\quad 88$

B Método de desacoplamento de hiperplanos na rede

C Equação de Bethe-Salpeter em coordenadas relativas 


\section{Capítulo 1}

\section{Introdução}

É hoje um consenso na comunidade que a Cromodinâmica Qüântica (QCD) é o melhor modelo elementar que temos para tratar as interações fortes ou nucleares.

Incorporando em sua base o modelo de quarks como as partículas fermiônicas com spin $1 / 2$ constituintes dos hádrons (mésons e bárions), este modelo é formulado como uma teoria de calibre, com campos de calibre (glúons) definidos na álgebra de Lie SU(3). Quarks e glúons são portadores de cargas de cor, e a dinâmica entre eles é formulada similarmente à dinâmica entre elétrons e o fóton no modelo de calibre com invariância U(1) que é a Eletrodinâmica Quântica. A grande diferença provém no fato de os glúons interagirem entre si, visto que a simetria de calibre da QCD é não-abeliana. Esta interação contém termos locais cúbicos (com uma derivada) e quárticos nos campos de glúons.

A QCD foi proposta nos anos sessenta e sua credibilidade foi tomando sustentação nos anos setenta, com a prova da renormalizabilidade perturbativa de alguns modelos de calibre não-abelianas e a descoberta de que a QCD é assintoticamente livre na região ultra-violeta de momentos (pequenas distâncias), se o número $N_{f}$ de sabores (isospin) dos quarks é menor que 17.

A liberdade assintótica na QCD nos permite poder considerar uma formulação perturbativa na região de altas energias, e foi assim que muitos fenômenos puderam ser seguidamente descritos, previstos e verificados experimentalmente (por exemplo, efeitos de três e quatro jatos em espalhamento $e^{+} e^{-}$, a proposição de novos sabores de quarks e a a descoberta de novas partículas como $J / \Psi$, etc).

Por outro lado, se nos retringirmos à QCD formulada no contínuo, nos deparamos com enormes dificuldades conceituais e técnicas na região de baixas (e médias!) energias. Glúons e quarks nunca foram observados livremente na natureza e a blindagem gluônica e o confinamento dos quarks foram postulados e aparecem no comportamento ultra singular do modelo na região infra-vermelho e nossa consequente dificuldade em explorar seu conteúdo neste domínio. 
Uma maneira de contornar este problema é a formulação do modelo na rede (reticulado), também iniciada nos anos setenta e que é hoje amplamente empregada para simulações numéricas bastante sofisticadas [17, 40].

É interessante observar que o formalismo numa rede com volume finito foi o ponto de partida para a demonstração nos anos noventa da renormalizabilidade ultra-violeta (limite do contínuo) do modelo de calibre SU(3) puro (sem quarks) [1, 18]. Tal prova é baseada em métodos analíticos rigorosos envolvendo um mapeamento do tipo grupo de renormalização, e considera o modelo completo, indo além da série perturbativa formal que pode (e provavelmente deve!) ser divergente.

É usando a formulação Hamiltoniana na rede, no chamado limite de acoplamento forte (ver abaixo), que o espectro de alguns hádrons foram primeiramente obtidos e suas massas calculadas em função do parâmetro da rede (distância típica entre pontos vizinhos). Foi também neste contexto que um potencial confinante linear no perímetro de plaquetas na rede (circuitos orientados fechados) foi derivado, sustentando a hipótese do confinamento.

Apesar disso, os sucessos do modelo no domínio de baixas energias são ainda muito aquém do esperado. Ainda pouco é conhecido, mesmo que formalmente, sobre o espectro de partículas do modelo. Sobretudo, devemos salientar a existência de uma grande lacuna que é a conexão da QCD com o conhecimento já adquirido em física nuclear. Muitas questões estão ainda por serem respondidas. Por exemplo, como compreender - a partir de primeiros princípios - o potencial fenomenológico de Yukawa, descrevendo interações entre campos efetivos de hádrons? O que mais, além de hádrons, contém a parte baixa do espectro de partículas? Estados ligados tal como o dêuteron? Etc...

O trabalho apresentado nesta tese é uma seqüência do trabalho introduzido nas referências [11, 12], visando responder a algumas destas questões básicas. Além de passar por uma análise rigorosa do espectro de um hádron ("gap" espectral), um méson e um bárion, as propriedades analíticas das curvas de dispersão associadas, simetrias e as eventuais aberturas de massa, consideramos a existência de estados ligados de dois hádrons até o limiar de duas partículas. Mais particularmente, continuando a análise para a existência de bárions e estados ligados bárion-bárion iniciada em [13], consideramos a existência de estados ligados méson-méson e méson-bárion em alguns modelos de QCD na rede, no regime de acoplamento forte.

Mesmo estando com parâmetros longe do limite físico (limite de escala), como aconteceu para o estudo do confinamento, nossa esperança inicial é que o modelo ainda deva preservar suas propriedades principais e que o regime de acoplamento forte não esteja associado a uma limitação muito drástica.

Vamos agora definir o modelo de calibre-matéria que estudaremos nesta Tese. Nós consideramos a formulação integral funcional de (Teoria Quântica de Campos) TQC com 
tempo imaginário, i.e. formulação Euclideana. A função de partição do nosso modelo é dada formalmente por

$$
Z=\int e^{-S(\psi, \bar{\psi}, g)} d \psi d \bar{\psi} d \mu(g)
$$

e para uma função $F(\bar{\psi}, \psi, g)$, as médias normalizadas são denotadas por

$$
\langle F(\bar{\psi}, \psi, g)\rangle=\frac{1}{Z} \int F(\bar{\psi}, \psi, g) e^{-S(\psi, \bar{\psi}, g)} d \psi d \bar{\psi} d \mu(g)
$$

Nesta tese consideramos os casos onde a dimensão espacial é $d=2,3$. Mais especificamente, nomeando por 0 a direção temporal, a rede é dada por $\mathbb{Z}_{o}^{d+1}$, onde $u=\left(u^{0}, \vec{u}\right)=$ $\left(u^{0}, u^{1}, \ldots, u^{d}\right) \in \mathbb{Z}_{o}^{d+1} \equiv \mathbb{Z}_{1 / 2} \times \mathbb{Z}^{d}$, onde $\mathbb{Z}_{1 / 2}=\{ \pm 1 / 2, \pm 3 / 2, \ldots\}$. A escolha da translação na direção temporal, evitando a coordenada cujo tempo é zero, é tal que, no limite do contínuo, os limites laterais das correlações de campos de Fermi podem ser acomodados. Em cada sítio $u \in \mathbb{Z}_{o}^{d+1}$, associamos campos fermiônicos, representados por variáveis de Grassmann, $\psi_{\alpha, a}(u)$, associado com um quark, e $\bar{\psi}_{\alpha, a}(u)$, associado com um anti-quark, que portam um índice de spin de Dirac $\alpha=1,2,3,4$, um índice de cor $a=1,2,3$. Vamos nos referir a $\alpha=1,2$ como índices "upper" e $\alpha=3,4$ como "lower", denotados respectivamente por $u$ e $\ell$. Temos também, denotando por $e^{\rho}, \rho=0,1, \ldots, d$, os vetores unitários na rede na direção $\rho$, para cada elo orientado na rede $<u, u \pm e^{\rho}>$ existe uma matrix $U\left(g_{u, u \pm e^{\rho}}\right) \in \mathrm{SU}(3)$ parametrizada pelo elemento do grupo de calibre $g_{u, u \pm e^{\rho}}$ e satisfazendo $U\left(g_{u, u+e^{\rho}}\right)^{-1}=U\left(g_{u+e^{\rho}, u}\right)$. Associada a cada plaqueta (cicuito fechado na rede) orientada na rede $p$ existe uma variável de plaqueta $\chi\left(U\left(g_{p}\right)\right)$ onde $U\left(g_{p}\right)$ é o produto orientado de matrizes de $\mathrm{SU}(3)$ para cada elo orientado da plaqueta e $\chi$ é o traço. A ação do modelo é dada por

$$
\begin{aligned}
S(\psi, \bar{\psi}, g)= & \frac{\kappa}{2} \sum \bar{\psi}_{\alpha, a}(u) \Gamma_{\alpha \beta}^{\epsilon e^{\rho}}\left(g_{u, u+\epsilon e^{\rho}}\right)_{a b} \psi_{\beta, b}\left(u+\epsilon e^{\rho}\right) \\
& +\sum_{u \in \mathbb{Z}_{o}^{d+1}} \bar{\psi}_{\alpha, a}(u) M_{\alpha \beta} \psi_{\beta, a}(u)-\frac{1}{g_{0}^{2}} \sum_{p} \chi\left(g_{p}\right),
\end{aligned}
$$

onde a primeira soma é feita sobre $u \in \mathbb{Z}_{o}^{d+1}, \epsilon= \pm 1, \rho=0,1, \ldots, d$ e sobre os índices repetidos. Por simplicidade de notação, algumas vezes omitimos $U$ de $U(g)$. Considerando os parâmetros, tomamos $m>0$, e o acoplamento quark-calibre ou parâmetro de hopping $\kappa>0$. Temos também, $g_{0}>0$ que descreve o campo de calibre puro e $M \equiv M(m, \kappa)=(m+2 \kappa) I_{4}$, sendo $I_{4}$ a matrix identidade $4 \times 4$. Também, dentro da família de ações de [35], temos $\Gamma^{ \pm e^{\rho}}=-I_{4} \pm \gamma^{\rho}$. Para $d=3, \gamma^{\rho}$ são as matrizes de Dirac $4 \times 4$ (hermitianas, de traço nulo e que anti-comutam

$$
\gamma^{0}=\left(\begin{array}{cc}
I & 0 \\
0 & -I
\end{array}\right) \text { e } \gamma^{j}=\left(\begin{array}{cc}
0 & i \sigma^{j} \\
-i \sigma^{j} & 0
\end{array}\right)
$$


e $\sigma^{j}, j=1,2,3$, denota as matrizes de Pauli $2 \times 2$, i.e.

$$
\sigma^{1}=\left(\begin{array}{ll}
0 & 1 \\
1 & 0
\end{array}\right), \quad \sigma^{2}=\left(\begin{array}{cc}
0 & -i \\
i & 0
\end{array}\right) \text { e } \sigma^{3}=\left(\begin{array}{cc}
1 & 0 \\
0 & -1
\end{array}\right)
$$

Para $d=2$ e $(3+1)$ dimensões, todas menos a matrix $\gamma^{3}$ aparece na ação. Matrizes de spin do tipo Dirac $4 \times 4$ serão usadas no Capítulo 2. Ao contrário, para simplificar nossa análise do ponto de vista algébrico, nos Capítulos 3 e 4, empregaremos matrizes de spin de Pauli $2 \times 2$. Neste caso, $I_{4}$ acima deve ser substituído por $I_{2}$, as somas sobre $\alpha$ e $\beta$ variam de 1 a 2 e adotamos $\gamma^{0}=\sigma^{3}$ e $\gamma^{1,2}=\sigma^{1,2}$ para as matrizes gama.

Continuando a explicar a função de partição definida na eq. (1.1), temos que a medida $d \mu(g)$ é o produto de medidas de Haar de SU(3) normalizadas sobre elos não-orientados (veja [37]) e as integrais sobre os campos de Grassmann são definidas de acordo com [3]. Para um polinômio nas variáveis de Grassmann com coeficientes dependendo das variáveis de calibre, a integral fermiônica é definida como o coeficiente do monômio de máximo grau, i.e. de $\prod_{u, \alpha, a} \bar{\psi}_{\alpha, a}(u) \psi_{\alpha, a}(u)$. Na eq. (1.1), $d \psi d \bar{\psi}$ significa $\prod_{u, \alpha, a, f} d \psi_{\alpha, a, f}(u) d \bar{\psi}_{\alpha, a, f}(u)$ tal que, com a normalização $\mathcal{N}=\langle 1\rangle$, temos

$$
\begin{aligned}
\left\langle\psi_{\alpha, a}(x) \bar{\psi}_{\beta, a}(y)\right\rangle^{(0)} & =(1 / \mathcal{N}) \int \psi_{\alpha, a}(x) \bar{\psi}_{\beta, b}(y) e^{-\sum_{u} \bar{\psi}_{\alpha^{\prime}, a^{\prime}}(u) M_{\alpha^{\prime} \beta^{\prime}} \psi_{\beta^{\prime}, a^{\prime}}(u)} d \psi d \bar{\psi} \\
& =M_{\alpha \beta}^{-1} \delta_{a b} \delta(x, y)
\end{aligned}
$$

com um delta de Kronecker para as coordenadas espaço-temporais. Com nossas restrições nos parâmetros, existe um espaço de Hilbert $\mathcal{H}$ de estados físicos (veja descrição detalhada abaixo), para $\kappa>0$; e a condição $m>0$ nos garante que as curvas de dispersão para uma partícula fermiônica livre cresce em cada componente positiva do momento.

Em toda esta tese, trabalhamos no regime de acoplamento forte, ou seja, tomamos o parâmetro de "hopping" suficientemente pequeno, $0<g_{0}^{-2}<<\kappa \lll 1$, e sem perda de generalidade, dado $\kappa$, escolhemos $m$ tal que $M=1$ na ação (1.2).

Uma importante característica da ação (1.2) é sua invariância sob transformações de calibre dadas por, para $u \in \mathbb{Z}_{o}^{d+1}$ e $h(u) \in \mathrm{SU}(3)$,

$$
\begin{gathered}
\psi(u) \mapsto h(u) \psi(u) \\
\bar{\psi}(u) \mapsto \bar{\psi}(u)[h(u)]^{-1} \\
U\left(g_{u+e^{\rho}, u}\right) \mapsto\left[h\left(u+e^{\rho}\right)\right]^{-1} U\left(g_{u+e^{\rho}, u}\right) h(u) .
\end{gathered}
$$

Outras simetrias da ação (1.2), tais como conjugação de carga, paridade, reflexões nas coordenadas, e rotações espaciais, são consideradas no Capítulo 2.

Nós agora passamos à consideração de dois pontos técnicos essenciais, a existência do limite termodinâmico na região de parâmetros considerada e a construção do espaço de 
Hilbert físico subjacente. Primeiro, para acoplamentos suficientemente pequenos $\kappa$ e $g_{0}^{-2}$, pelo método de expansão em polímeros, (veja [35, 36, 23]), o limite termodinâmico das funções de correlação existem e as funções de correlação truncadas tem decaimento em árvore. Como consequência da expansão em polímeros as funções de correlação estendem a funções analíticas nos parâmetros de acoplamento $\kappa$ e $g_{0}^{-2}$. Para a expansão formal no parâmetro hopping, ou expansão de acoplamento forte, veja $[28,5]$.

Segundo, no nosso caso, uma fórmula de F-K proporciona a construção de um espaço de Hilbert, que designaremos por $\mathcal{H}$. Denotando $T_{0}^{x^{0}}, T_{i}^{x^{i}}, i=1,2, \ldots, d$, as translações das funções nas variáveis de Grassmann e calibre para $x^{0} \geq 0, x=\left(x^{0}, \vec{x}\right) \in \mathbb{Z}^{d+1}$; e para $F$ e $G$ dependendo somente das coordenadas com $u^{0}=1 / 2$, temos a fórmula de Feynman-Kac (F-K)

$$
\left(G, \check{T}_{0}^{x^{0}} \check{T}_{1}^{x^{1}} \ldots \check{T}_{d}^{x^{d}} F\right)_{\mathcal{H}}=\left\langle\left[T_{0}^{x^{0}} T_{1}^{x^{1}} \ldots T_{d}^{x^{d}} F\right] \Theta G\right\rangle
$$

que relaciona produtos internos no espaço de Hilbert $\mathcal{H}$ com médias de Gibbs, onde $\Theta$ é um operador anti-linear que envolve reflexão no tempo. Seguindo [35], com a convenção usual da soma sobre índices repetidos, a ação de $\Theta$ em campos simples é dada por

$$
\begin{aligned}
& \Theta \bar{\psi}_{\alpha, a}(u)=\left(\gamma^{0}\right)_{\alpha \beta} \psi_{\beta, a}(t u), \\
& \Theta \psi_{\alpha, a}(u)=\bar{\psi}_{\beta, a}(t u)\left(\gamma^{0}\right)_{\beta \alpha}
\end{aligned}
$$

onde $t\left(u^{0}, \vec{u}\right)=\left(-u^{0}, \vec{u}\right)$, para $A$ e $B$ monômios, $\Theta(A B)=\Theta(B) \Theta(A)$; e para uma função dos campos de calibre $\Theta f\left(\left\{g_{u v}\right\}\right)=f^{*}\left(\left\{g_{(t u)(t v)}\right\}\right), u, v \in \mathbb{Z}_{o}^{d+1}$, onde $*$ significa conjugação complexa. $\Theta$ estende por anti-linearidade para a álgebra dos campos de calibre e variáveis de Grassmann. Como operadores lineares em $\mathcal{H}, \check{T}_{\rho}, \rho=0,1, \ldots, d$, comutam mutuamente; $\check{T}_{0}$ é auto-adjunto, com $-1 \leq \check{T}_{0} \leq 1$, e $\check{T}_{j=1, \ldots, d}$ são unitários, tal que, podemos escrever $\check{T}_{j}=e^{i P^{j}}$ e $\vec{P}=\left(P^{1}, \ldots, P^{d}\right)$ é o operador de momento auto-adjunto, com pontos espectrais $\vec{p} \in \mathbf{T}^{d} \equiv(-\pi, \pi]^{d}$. Como $\check{T}_{0}^{2} \geq 0$, definimos o operador energia $H \geq 0$ por $\check{T}_{0}^{2}=e^{-2 H}$. Referimo-nos a pontos no espectro de energia-momento associados a momento espacial zero como massa. A condição de positividade $\langle F \Theta F\rangle \geq 0$ é estabelecida em [35], mas podem existir $F$ 's não nulos tais que $\langle F \Theta F\rangle=0$. A coleção de tais $F$ 's é denotada por $\mathcal{N}$. Então, um pré-espaço de Hilbert $\mathcal{H}^{\prime}$ pode ser construído a partir do produto interno $\langle G \Theta F\rangle$. O espaço de Hilbert físico $\mathcal{H}$ é definido como o completamento do espaço quociente $\mathcal{H}^{\prime} / \mathcal{N}$.

Basicamente, existem duas abordagens usadas no estudo rigoroso do espectro de partículas em teorias na rede. Uma delas é baseada em métodos remanescentes de teorias de campo no contínuo (e.g. decoplamento de hiperplanos, subtração Euclideana) [38, 9, 31, 36, 33, 10] e a outra usa métodos de mecânica estatística (e.g. superfícies aleatórias) [4, 22]. Nesta tese, seguimos a primeira abordagem.

No Capítulo 2, consideramos a existência de mésons em $(2+1)$ e $(3+1)$-dimensões, vistos 
como estados ligados de um quark e um anti-quark. Considerando um modelo mais realista que em [11], trabalhamos com matrizes de spin de Dirac $4 \times 4$ como em [12]. Esta escolha também nos facilita a análise de abertura de massa para os mésons no modelo. Aqui, denotamos por $\mathcal{H}_{e} \subset \mathcal{H}\left(\mathcal{H}_{o} \subset \mathcal{H}\right)$ o subespaço gerado por um número par (ímpar) de férmions. Mostramos que mésons existem, com massas assintóticas $-2 \ln \kappa$, manifestados pelo aparecimento de curvas de dispersão isoladas no espectro energia-momento (lacuna inferior da massa) e é o único espectro no subespaço $\mathcal{H}_{e} \subset \mathcal{H}$, até o limiar de dois-mésons que é assintoticamente da ordem $-4 \ln \kappa$. Nosso método de obtenção do espectro de uma patícula é baseado numa análise da função de dois pontos $(\operatorname{com} M, L \in \mathcal{H})$ onde * é o complexo conjugado e $\chi$ a função característica

$$
G_{M, L}= \pm\langle(\Theta M)(x) L(y)\rangle \chi_{x^{0} \geq y^{0}}+\langle M(x)(\Theta L)(y)\rangle^{*} \chi_{x^{0}<y^{0}}
$$

que admite a seguinte representação espectral ( $\mathcal{E}$ sendo o produto conjunto das famílias espectrais dos operadores de energia e momento)

$$
G_{M, L}(u, v)=\int_{-1}^{1} \int_{\mathbf{T}^{d}}\left(\lambda^{0}\right)^{\left|v^{0}-u^{0}\right|-1} e^{i \vec{\lambda} \cdot(\vec{v}-\vec{u})} d\left(M, \mathcal{E}\left(\lambda^{0}, \vec{\lambda}\right) L\right)_{\mathcal{H}}
$$

análoga à representação de Källen-Lehman em TQC no contínuo. Enfatizamos que é esta representação espectral que nos permite identificar singularidades para momento complexo da transformada de Fourier da função de dois-pontos com pontos no espectro de energia-momento. Tanto quanto sabemos, a representação espectral dada pela eq. (1.7) não existia na literatura anteriormente aos nossos resultados. Gostaríamos de mencionar que a determinação da taxa de decaimento exponencial das funções de correlação, tal qual é feita em simulações numéricas e outros trabalhos se servindo de formulação Hamiltoniana, não é suficiente para identificarmos estas taxas como espectro de massa [22]. Isto é especialmente verdade no caso em que multiplicidades estão envolvidas e há pequenas aberturas de massa. Para determinarmos o espectro de massa, é essencial conhecermos o comportamento de $G$ e $\Gamma$, a inversa convolutiva de $G$, a curtas e longas distâncias. O comportamento a longas distâncias é obtido através de cotas para o decaimento de $G$ e $\Gamma$, usando o método conhecido como desacoplamento de hiperplanos. Mais detalhes serão dados adiante.

No Capítulo 3, tomando $d=2$ e escolhendo por simplicidade trabalhar com matrizes de spin de Pauli $2 \times 2$, consideramos a existência de estados ligados de duas partículas: dois mésons e um bárion e um méson, para o caso de um sabor. Seguindo [15] e ainda trabalhando em $d=2$ e com matrizes de spin de Pauli $2 \times 2$, mas agora com dois sabores de quarks (caso isospin), no Capítulo 4, consideramos a existência de estados ligados de dois mésons.

Nosso método de determinação dos estados ligados é baseado na equação de Bethe 
Salpeter (B-S) na rede que é dada por, em termos operadores,

$$
D=D_{0}+D K D_{0}
$$

ou em termos de núcleos, no que chamamos de representação para tempos iguais (i.e. $x_{1}^{0}=x_{2}^{0}$ e $\left.x_{3}^{0}=x_{4}^{0}\right)$

$$
\begin{aligned}
& D\left(x_{1}, x_{2}, x_{3}, x_{4}\right)=D_{0}\left(x_{1}, x_{2}, x_{3}, x_{4}\right)+ \\
& \int D_{0}\left(x_{1}, x_{2}, y_{1}, y_{2}\right) K\left(y_{1}, y_{2}, y_{3}, y_{4}\right) D\left(y_{3}, y_{4}, x_{3}, x_{4}\right) \\
& \times \delta\left(y_{1}^{0}-y_{2}^{0}\right) \delta\left(y_{3}^{0}-y_{4}^{0}\right) d y_{1} d y_{2} d y_{3} d y_{4},
\end{aligned}
$$

onde

$$
D_{0}\left(x_{1}, x_{2}, x_{3}, x_{4}\right)=G_{2}\left(x_{1}, x_{3}\right) G_{2}\left(x_{2}, x_{4}\right)+G_{2}\left(x_{1}, x_{4}\right) G_{2}\left(x_{2}, x_{3}\right) .
$$

$D$ é a função de quatro pontos pacialmente truncada, i.e. $D\left(x_{1}, x_{2}, x_{3}, x_{4}\right)=G_{4}\left(x_{1}, x_{2}, x_{3}, x_{4}\right)-$ $G_{2}\left(x_{1}, x_{3}\right) G_{2}\left(x_{2}, x_{4}\right)$ e $D_{0}$ corresponde à subtração das contribuições desconexas. $K$ é definido pela equação acima e é dado por $D_{0}^{-1}-D^{-1}$. Esta equação exibe uma característica marcante: a irredutibilidade por duas partículas. Isto quer dizer que, na expansão perturbativa de $K$ em função do parâmetro $\kappa$ teremos somente contribuições conexas (i.e. conectando os pontos $x_{1}, x_{2}, x_{3}$ e $x_{4}$ ). Esta propriedade é importante levando ao decaimento apropriado de $K$ em função do parâmetro $\kappa$. Nosso método de resolução desta equação consiste em adotarmos a aproximação de ordem mais baixa não nula em $\kappa$ para $K$, que chamamos de aproximação escada ("ladder") e a denotaremos por $L$. Isto é feito depois de escrevermos a equação de Bethe-Salpeter em coordenadas relativas. Definimos as coordenadas relativas, i.e., $\xi=x_{2}-x_{1}$, $\eta=x_{4}-x_{3}$ e $\tau=x_{3}-x_{2}$ análogas às coordenadas reduzida e do centro de massa no contínuo. Ao final de nossa análise estabelecemos uma comparação da equação de B-S em coordenadas relativas e a equação de Schrödinger na forma resolvente, onde relembramos

$$
(H-z)^{-1}=\left(H_{0}-z\right)^{-1}-\lambda\left(H_{0}-z\right)^{-1} V(H-z)^{-1},
$$

$H=H_{0}+\lambda V, H_{0}=-\Delta / 2\left[-\Delta\right.$ é o Laplaciano em $\left.\ell_{2}\left(\mathbb{Z}^{d}\right)\right]$ e $V$ é o potencial de interação.

Esta analogia nos permite entender o mecanismo de interação entre os constituintes dos estados ligados que analisamos, i.e., qual o potencial associado a interação entre dois mésons e um méson e um bárion?

Nossos resultados na aproximação escada, para o modelo em $2+1$ dimensões, matrizes de spin $2 \times 2$ e com um sabor de quark mostram que estados ligados de dois mésons ou um méson e um bárion não estão presentes no espectro de energia-momento até o limiar de duas partículas. Nossa interpretação para a não ligação das duas partículas está no potencial repulsivo advindo da exclusão de Pauli, que não é favorável a aproximação de quarks em estados idênticos. Ao contrário, aumentando o volume do espaço de configurações para os 
quarks, se o número de sabores é dois e restringindo nossa análise ao subespaço par $\mathcal{H}_{e}$, no setor de isospin total zero, mostramos que existe um estado ligado méson-méson com energia de ligação aproximada $0,02359 \kappa^{2}$. Nossa análise também revela que o potencial atrativo de ligação entre os dois mésons, envolvendo uma troca de partícula e alcance um, é bastante dependente de certas correlações de campos de calibre. Um estado ligado bárion-bárion para o mesmo modelo foi detectado em [15].

O problema completo, isto é a análise do padrão espectral além da aproximação escada é obtida como em [33] perturbando $L$ com um termo cuja ordem do parâmetro $\kappa$ é imediatamente superior e considerando a equação de B-S com esta correção. Desta forma, é possível mostrar que os resultados espectrais para $D$ são preservados. Convém ressaltarmos que o decaimento em $\kappa$ obtido para $K$ pelo método de desacoplamento de hiperplanos desempenha um papel fundamental na demonstração deste resultado. Nossos resultados devem poder ser estendidos para a análise do modelo completo (além da aproximação escada) seguindo a abordagem de $[9,10,33]$, já que nossos trabalhos incluem todos ingredientes principais para esta extensão. Além disso, nossos resultados correspondem a um primeiro passo para a análise do espectro de QCD na rede em dimensão $3+1$ e diversos sabores de quarks.

Para complementar o texto e facilitar sua leitura, incluímos três apêndices. O Apêndice A descreve o procedimento de Creutz para calcular integrais de calibre $[5,6,7,8]$. Nosso objetivo, ao escrever este apêndice, é apresentar didaticamente o procedimento introduzido por Creutz. O Apêndice B descreve detalhadamente o método de desacoplameto de hiperplanos usado para obtermos o decaimento das funções de correlação envolvidas (dois e quatro pontos). O Apêndice C apresenta a obtenção da equação de Bethe-Salpeter em coordenadas relativas a partir de sua representação em tempos iguais. 


\section{Capítulo 2}

\section{Existência de mésons em $(2+1)$ e $(3+1)$ dimensões, um sabor e matrizes de Dirac $4 \times 4$}

Neste capítulo, como em [12], considerando o caso de um sabor, $(2+1)$ e $(3+1)$ dimensões, com matrizes de Dirac $4 \times 4$, apresentamos nossos resultados espectrais para uma partícula no setor mesônico. Na Seção 2.1, consideramos simetrias do modelo (1.2) outras além da invariância de calibre dada pela eq. (1.5) obtidos em [12]. Definimos a função de dois pontos para o méson, introduzimos uma representação espectral geral para vetores no espaço de Hilbert físico $\mathcal{H}$, que desempenhará um papel fundamental ao longo desta tese. Apresentamos nossos resultados a respeito da existência de mésons e resultados a respeito da existência de bárions obtidos em [12]. Na Seção 2.2, descrevemos o comportamento de $G$ e sua inversa por convolução $\Gamma$ a curtas e longas distâncias (obtido pelo método de desacoplamento de hiperplanos (Apêndice B)). Na Seção 2.3, demonstramos o principal resultado deste capítulo, enunciado no Teorema 5 que consiste na determinação do padrão espectral no setor mesônico até o limiar de dois mésons $-4 \ln \kappa$. Finalizamos este capítulo estendendo nossos resultados de $\mathcal{H}_{m}$ (subespaço de $\mathcal{H}$ gerado pelos campos mesônicos das eqs. (2.4) e (2.5)) para $\mathcal{H}_{e}$ (subespaço gerado por um número par de campos fermiônicos) usando o método de subtrações Euclideanas [31].

Os resultados deste capítulo foram publicados em [19]. 


\subsection{Apresentação dos resultados sobre a existência de um méson e um bárion}

Antes de apresentarmos nossos resultados vamos considerar simetrias da ação (1.2), além da invariância por transformações de calibre dadas pela eq. (1.5), e apresentar importantes resultados a respeito de simetrias obtidos em [12]. Operações de simetria são definidas na álgebra dos campos (de calibre ou Grassmannianos). Uma operação de simetria $\mathbb{Y}$ leva campos Grassmannianos simples para

$$
\begin{aligned}
\mathbb{Y} \psi_{\alpha, a}(x) & =A_{\alpha \beta} \tilde{\psi}_{\beta, a}(\mathcal{Y} x) \\
\mathbb{Y} \bar{\psi}_{\alpha, a}(x) & =\tilde{\bar{\psi}}_{\beta, a}(\mathcal{Y} x) B_{\beta \alpha}
\end{aligned}
$$

e age em funções $f\left(g_{x z}\right)$ do grupo de calibre $\mathrm{SU}(3)$ por

$$
\mathbb{Y} f\left(g_{x z}\right)=(\mathbb{Y} f)\left(g_{\mathcal{Y} x \mathcal{Y}_{z}}\right)
$$

onde $\mathcal{Y}$ é uma transformação agindo nas coordenadas, $\sim$ significa barra ou sem barra e Y age nos campos de calibre. Em monômios Grassmannianos $M_{1}, M_{2}$, $\mathbb{Y}$ tem a propriedade

$$
\mathbb{Y}\left(M_{1} M_{2}\right)=\mathbb{Y}\left(M_{1}\right) \mathbb{Y}\left(M_{2}\right)
$$

que identificamos como preservar a ordem (homomorfismo), ou

$$
\mathbb{Y}\left(M_{1} M_{2}\right)=\mathbb{Y}\left(M_{2}\right) \mathbb{Y}\left(M_{1}\right)
$$

que identificamos como reverter a ordem (anti-homomorfismo). As operações de simetria são estendidas para toda a álgebra dos campos por linearidade ou anti-linearidade.

Uma simetria do modelo é definida como uma operação de simetria que deixa a ação $S$ da eq. (1.2) invariante, e que tem a seguinte propriedade envolvendo as médias normalizadas $F$ invariantes por transformações de calibre:

$$
\langle\mathbb{Y} F\rangle=\langle F\rangle^{\sharp}
$$

onde $\langle F\rangle^{\sharp}$ significa ou $\langle F\rangle$ ou $\langle F\rangle^{*}$.

Ainda mais, a simetria pode ser implementada no espaço de Hilbert $\mathcal{H}$ por um operador linear ou anti-linear estabilizando o espaço nulo $\mathcal{N}$, i.e. tal que se $F \in \mathcal{N}$ então $\mathbb{Y} F \in \mathcal{N}$. (Veja [12].)

Nós usaremos neste trabalho os resultados do seguinte Teorema provado em [12].

Teorema 1 Considerando matrizes de Dirac $4 \times 4, \gamma_{i}(i=1,2,3)$ cada um das operações de 
simetria especificadas abaixo é uma simetria do modelo.

$\underline{O \text { caso } d=3}$ :

1. Rotações espaciais $r$ por $\pi / 2$ e $\pi$ em relação a:

(a) A direção $e^{1}: r_{1}\left(e^{1}, e^{2}, e^{3}\right)=\left(e^{1}, e^{3},-e^{2}\right)$, com $B=\frac{e^{i \pi / 4}}{\sqrt{2}}\left(1-\gamma^{2} \gamma^{3}\right)=A^{-1}, e$ $f\left(g_{x y}\right) \mapsto f\left(g_{r_{1} x r_{1} y}\right)$. Para rotações de $\pi, B=-i \gamma^{2} \gamma^{3}$.

(b) $A$ direção $e^{2}: r_{2}\left(e^{1}, e^{2}, e^{3}\right)=\left(-e^{3}, e^{2}, e^{1}\right)$, com $B=\frac{e^{i \pi / 4}}{\sqrt{2}}\left(1-\gamma^{3} \gamma^{1}\right)=A^{-1}, e$ $f\left(g_{x y}\right) \mapsto f\left(g_{r_{2} x r_{2} y}\right)$. Para rotações de $\pi, B=-i \gamma^{3} \gamma^{1}$.

(c) $A$ direção $e^{3}: r_{3}\left(e^{1}, e^{2}, e^{3}\right)=\left(e^{2},-e^{1}, e^{3}\right)$, com $B=\frac{e^{i \pi / 4}}{\sqrt{2}}\left(1-\gamma^{1} \gamma^{2}\right)=A^{-1}, e$ $f\left(g_{x y}\right) \mapsto f\left(g_{r_{3} x r_{3} y}\right)$. Para rotações de $\pi, B=-i \gamma^{1} \gamma^{2}$.

2. Reflexões nas coordenadas espaciais $\mathcal{R}$ in:

(a) A coordenada $e^{1}: \mathcal{R}_{1}\left(e^{1}, e^{2}, e^{3}\right)=\left(-e^{1}, e^{2}, e^{3}\right)$, com $B=i \gamma^{0} \gamma^{2} \gamma^{3}=A^{-1}$, tal que $f\left(g_{x y}\right) \mapsto f\left(g_{\mathcal{R}_{1} x \mathcal{R}_{1} y}\right)$.

(b) A coordenada $e^{2}: \mathcal{R}_{2}\left(e^{1}, e^{2}, e^{3}\right)=\left(e^{1},-e^{2}, e^{3}\right)$, com $B=i \gamma^{0} \gamma^{1} \gamma^{3}=A^{-1}$, tal que $f\left(g_{x y}\right) \mapsto f\left(g_{\mathcal{R}_{2} x \mathcal{R}_{2} y}\right)$.

(c) A coordenada $e^{3}: \mathcal{R}_{3}\left(e^{1}, e^{2}, e^{3}\right)=\left(e^{1}, e^{2},-e^{3}\right)$, com $B=-i \gamma^{0} \gamma^{1} \gamma^{2}=A^{-1}$, tal que $f\left(g_{x y}\right) \mapsto f\left(g_{\mathcal{R}_{3} x \mathcal{R}_{3} y}\right)$.

3. Paridade $\mathcal{P}$ : $\mathcal{P}\left(e^{1}, e^{2}, e^{3}\right)=\left(-e^{1},-e^{2},-e^{3}\right)$, com $B=\gamma^{0}=A^{-1}$, tal que $f\left(g_{x y}\right) \mapsto$ $f\left(g_{\mathcal{P} x \mathcal{P}_{y}}\right)$.

4. Conjugação de Carga $\mathcal{C}: \sim$ significa barra, $B=\gamma^{2} \gamma^{0}, f\left(g_{x y}\right) \mapsto f\left(g_{x y}^{*}\right)$.

5. Reversão no tempo $\mathcal{T}: \mathcal{T}\left(e^{0}, e^{1}, e^{2}, e^{3}\right)=\left(-e^{0}, e^{1}, e^{2}, e^{3}\right), B=\gamma^{0}, \sim$ significa barra, $\sharp$ significa complexo conjugado, $f\left(g_{x y}\right) \mapsto f\left(g_{\mathcal{T} x \mathcal{T} y}\right)^{*}$.

As operações $1-3$ preservão a ordem, 4-5 invertem a ordem, $1-4$ (respectivamente 5) são lineares (respectivamente, anti-lineares) e são implementedadas em $\mathcal{H}$ por operadores unitários lineares (respectivamente, anti-lineares).

O caso d $=2$ :

Obviamente, não temos rotações $r_{1}$ e $r_{2}$, e também reflexões em relação ao eixo $e^{3}$. As matrizes $A$ e $B$ são as mesmas definidas acima, exceto pela conjugação de carga, onde $A=\gamma^{1}=B$.

Usando o mesmo procedimento presente em [12] podemos mostrar resultados similares aos descritos acima quando matrizes de spin (Pauli) $2 \times 2$ estão presentes. 
Teorema 2 Em $(2+1)$ dimensões e considerando matrizes de spin $2 \times 2$ temos as seguintes simetrias

1. Rotações espaciais $r$ por $\pi / 2$ e $\pi$ :

(a) $r\left(e^{1}, e^{2}\right)=\left(e^{2},-e^{1}\right)$, com $B=\frac{e^{i \pi / 4}}{\sqrt{2}}\left(1-i \sigma^{3}\right)=A^{-1}$, e $f\left(g_{x y}\right) \mapsto f\left(g_{r_{3} x r_{3} y}\right)$. Para rotações de $\pi, B=\sigma^{3}$ (equivalente à simetria de paridade definida abaixo).

2. Reflexões nas coordenadas espaciais $\mathcal{R}$ :

(a) A coordenada $e^{1}: \mathcal{R}_{1}\left(e^{1}, e^{2}\right)=\left(-e^{1}, e^{2}\right)$, com $B=\sigma^{3}=A^{-1}$, tal que $f\left(g_{x y}\right) \mapsto$ $f\left(g_{\mathcal{R}_{1} x \mathcal{R}_{1} y}\right)$.

(b) A coordenada $e^{2}: \mathcal{R}_{2}\left(e^{1}, e^{2}\right)=\left(e^{1},-e^{2}\right)$, com $B=\sigma^{2}=A^{-1}$, tal que $f\left(g_{x y}\right) \mapsto$ $f\left(g_{\mathcal{R}_{2} x \mathcal{R}_{2} y}\right)$.

3. Paridade $\mathcal{P}: \mathcal{P}\left(e^{1}, e^{2}\right)=\left(-e^{1},-e^{2}\right)$, com $B=\sigma^{3}=A^{-1}$, tal que $f\left(g_{x y}\right) \mapsto f\left(g_{\mathcal{P}_{x} \mathcal{P}_{y}}\right)$.

4. Conjugação de Carga $\mathcal{C}: \sim$ significa barra, $B=\sigma^{2}, f\left(g_{x y}\right) \mapsto f\left(g_{x y}^{*}\right)$.

5. Reversão no tempo $\mathcal{T}: \mathcal{T}\left(e^{0}, e^{1}, e^{2}\right)=\left(-e^{0}, e^{1}, e^{2}\right), B=\sigma^{3}, \sim$ significa barra, $\sharp$ significa complexo conjugado, $f\left(g_{x y}\right) \mapsto f\left(g_{\mathcal{T} x \mathcal{T} y}\right)^{*}$.

A extensão destas operações para toda a ágebra é definida de forma similar ao Teorema 1.

Uma importante questão é qual a combinação certa de campos simples $(\psi$ e $\bar{\psi})$, para mésons um par $\psi \bar{\psi}$ e para bárions $\psi \psi \psi$ ou $\bar{\psi} \bar{\psi} \bar{\psi}$, deve ser adotada na definição das funções de correlação, no nosso caso, dois e quatro pontos. Nossa metodologia de determinação dos campos compostos, mésons e bárions, é essencialmente baseada no método conhecido como desacoplamento de hiperplanos que discutimos de forma detalhada no Apêndice B. Este método serve de guia, indicando quais as escolhas de campos compostos que devemos tomar para mostrarmos as excitações no espectro de energia-momento. Estas escolhas, conforme veremos, incorporam os dois ingredientes básicos de nossa análise, i.e., para a função de dois pontos obtemos o decaimento adequado (em função do parâmetro de "hopping" $\kappa$ ) para a inversa convolutiva de $G, \Gamma$. No caso da função de quatro pontos, com nossas escolhas, o núcleo de B-S, $K$, tem o decaimento adequado. Mais detalhes serão dados adiante.

Agora apresentamos nossos resultados sobre partículas mesônicas, suas massas e curvas de dispersão. Nós consideramos o subespaço $\mathcal{H}_{m} \subset \mathcal{H}$ gerado por vetores associados a variáveis de Grassmann e invariantes por transformaçoẽs de calibre dados por $\mu_{k}(u)$ e $\Pi_{l}(v)$ : 


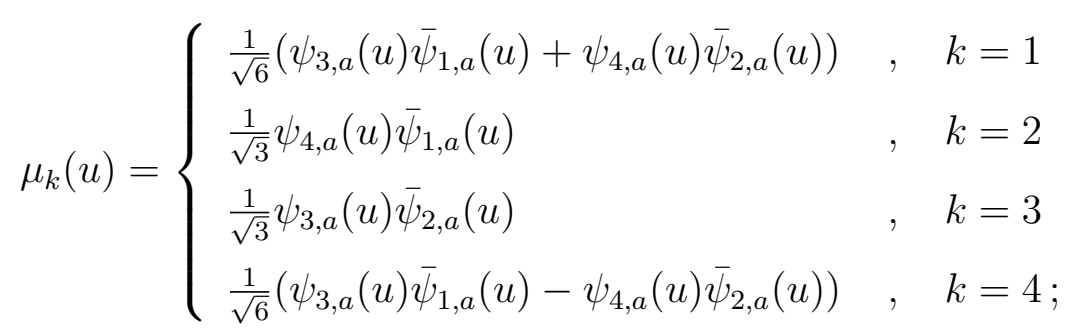

$\mathrm{e}$

$$
\Pi_{l}(v)= \begin{cases}\frac{1}{\sqrt{6}}\left(\bar{\psi}_{3, a}(v) \psi_{1, a}(v)+\bar{\psi}_{4, a}(v) \psi_{2, a}(v)\right) & , \quad l=1 \\ \frac{1}{\sqrt{3}} \bar{\psi}_{4, a}(v) \psi_{1, a}(v) & , \quad l=2 \\ \frac{1}{\sqrt{3}} \bar{\psi}_{3, a}(v) \psi_{2, a}(v) & , \quad l=3 \\ \frac{1}{\sqrt{6}}\left(\bar{\psi}_{3, a}(v) \psi_{1, a}(v)-\bar{\psi}_{4, a}(v) \psi_{2, a}(v)\right) & , \quad l=4\end{cases}
$$

As normalizações são escolhidas de tal forma que a função de dois pontos definida abaixo, em pontos coincidentes e parâmetro de "hopping" $\kappa=0$, é a matriz identidade denotada por $I_{4}$.

Para vermos a conexão com o espectro de energia-momento, definimos a função de dois mésons, estabelecemos uma fórmula de Feynman-Kac e uma representação espectral. A função de dois pontos pontos é definida por ( $\chi$ denota a função característica)

$$
\begin{aligned}
G_{k l}(u, v) & =\left\langle\mu_{k}(u) ; \Pi_{l}(v)\right\rangle_{T} \chi_{u^{0} \leq v^{0}}+\left\langle\Pi_{k}(u) ; \mu_{l}(v)\right\rangle_{T}^{*} \chi_{u^{0}>v^{0}} \\
& =\left\langle\mu_{k}(u) \Pi_{l}(v)\right\rangle \chi_{u^{0} \leq v^{0}}+\left\langle\Pi_{k}(u) \mu_{l}(v)\right\rangle^{*} \chi_{u^{0}>v^{0}}=G_{k l}(u-v),
\end{aligned}
$$

onde $\langle;\rangle_{T}$ é a função de correlação truncada

$$
\langle F(u) ; H(v)\rangle_{T}=\langle F(u) H(v)\rangle-\langle F(u)\rangle\langle H(v)\rangle .
$$

Na eq. (2.6) usamos que o truncamento parcial, com nossas escolhas dos campos mesônicos é zero, ou seja $\left\langle\Pi_{k}\right\rangle=\left\langle\mu_{k}\right\rangle=0$, pela simetria de paridade do Teorema 1.

A definição dada pela eq. (2.6) apresenta três características essenciais para nossa análise do padrão espectral no setor mesônico:

- A extensão para tempos iguais da definição (2.6) associada ao ordenamento temporal $u^{0}>v^{0}$ concorda com a relacionada a $u^{0} \leq v^{0}$, usando invariância por translação e reversão no tempo do Teorema 1.

- Sua transformada de Fourier admite uma representação espectral simples. [Veja a eq. $(2.9)$.]

- Ela nos permite mostrar a existência de partículas manifestada pela determinação de suas curvas de dispersão e massas (Teorema 5). 
A representação espectral da Proposição seguinte é uma ferramenta importante e desempenhará um papel fundamental ao longo deste trabalho. Sua importância reside no fato, conforme mencionamos no terceiro item acima, de estabelecer a conexão entre singularidades das funções de correlação com pontos no espectro de energia-momento.

Proposição 1 A seguinte função de dois pontos

$$
\mathcal{G}_{M, L}(x) \equiv \begin{cases} \pm\left\langle(\Theta M)\left(u^{0}\right) L\left(v^{0}, \vec{x}\right)\right\rangle & , u^{0} \leq v^{0} \\ \left\langle M\left(u^{0}\right)(\Theta L)\left(v^{0}, \vec{x}\right)\right\rangle^{*} & u^{0}>v^{0}\end{cases}
$$

onde, $L$ e $M$ são quaisquer dois vetores de $\mathcal{H}$, admite a representação espectral, com $v^{0} \neq u^{0}$

$$
\mathcal{G}_{M, L}(u, v)=\int_{-1}^{1} \int_{\mathbf{T}^{d}}\left(\lambda^{0}\right)^{\left|v^{0}-u^{0}\right|-1} e^{i \vec{\lambda} \cdot(\vec{v}-\vec{u})} d\left(M, \mathcal{E}\left(\lambda^{0}, \vec{\lambda}\right) L\right)_{\mathcal{H}},
$$

onde $\mathcal{E}\left(\lambda^{0}, \vec{\lambda}\right)=\mathcal{E}_{0}\left(\lambda^{0}\right) \mathcal{E}(\vec{\lambda})$, $\mathcal{E}_{0}\left(\lambda^{0}\right)$ é a família espectral para o operador $\check{T}_{0}$, e $\mathcal{E}(\vec{\lambda})=$ $\prod_{i=1}^{d} \mathcal{E}_{i}\left(\lambda^{i}\right)$ é o produto das famílias espectrais para $P^{i}$. Para $x \in \mathbb{Z}^{d+1}$, com um abuso de notação, definimos $\mathcal{G}_{M, L}(x=v-u) \equiv \mathcal{G}_{M, L}(u, v)$. Então, a transformada de Fourier de $\mathcal{G}_{M, L}(x)$ admite a representação espectral

$$
\begin{aligned}
\tilde{\mathcal{G}}_{M, L}(p)=\tilde{\mathcal{G}}_{M, L}(\vec{p})+(2 \pi)^{3} \int_{-1}^{1} \int_{\mathbf{T}^{d}} \delta(\vec{p}-\vec{\lambda}) f\left(\lambda^{0}, p^{0}\right) \\
\times d_{\lambda^{0}} d_{\vec{\lambda}}\left(M(1 / 2, \overrightarrow{0}), \mathcal{E}_{0}\left(\lambda^{0}\right) \mathcal{E}(\vec{\lambda}) L(1 / 2, \overrightarrow{0})\right)_{\mathcal{H}},
\end{aligned}
$$

onde $f(x, y)=\left(e^{i x}-y\right)^{-1}+\left(e^{-i x}-y\right)^{-1}$ e $\tilde{\mathcal{G}}_{M, L}(\vec{p})=\sum_{\vec{x} \in \mathbb{Z}^{d}} e^{-i \vec{p} \cdot \vec{x}} \mathcal{G}_{M, L}\left(x^{0}=0, \vec{x}\right)$.

Observação 1 Como não temos representação espectral para $v^{0}=u^{0}$ na eq. (2.8), separamos a contribuição $u^{0}=v^{0}$ que corresponde a $\tilde{\mathcal{G}}_{M, L}(\vec{p})$ na eq. (2.9).

Restringindo nossa análise ao setor de uma partícula mesônica obtemos:

Corolário 1 Para $u^{0} \neq v^{0}, \Pi_{k} \equiv \Pi_{k}(1 / 2, \overrightarrow{0})$, a seguinte fórmula de $F$-K é satisfeita, fazendo $M=\Pi_{k}$ e $L=\Pi_{l}$ em (2.8),

$$
G_{k l}(u, v) \equiv \mathcal{G}_{\Pi_{k}, \Pi_{l}}(u, v)=\int_{-1}^{1} \int_{\mathbf{T}^{d}}\left(\lambda^{0}\right)^{\left|v^{0}-u^{0}\right|-1} e^{i \vec{\lambda} .(\vec{v}-\vec{u})} d\left(\Pi_{k}, \mathcal{E}\left(\lambda^{0}, \vec{\lambda}\right) \Pi_{l}\right)_{\mathcal{H}}
$$

e é uma função par de $\vec{v}-\vec{u}$.

Observação 2 Pela representação espectral da eq. (2.10) vemos que os campos $\Pi$ geram particulas e os campos $\mu$ da definição (2.4) são campos auxiliares que entram na definição da função de dois pontos (2.6). 
Antes de apresentarmos nossos resultados (enunciados nos Teoremas 3, 4 e 5 ) descrevemos o procedimento utilizado para determinarmos as massas e as curvas de dispersão para um méson abaixo do limiar de dois mésons $-4 \ln \kappa$. Considerando a inversa convolutiva de $G$, $\Gamma$, definida pela seguinte série de Neumann

$$
\Gamma=\sum_{i=0}^{\infty}(-1)^{i}\left[G_{d}^{-1} G_{n}\right]^{i} G_{d}^{-1}
$$

onde $G_{d}$ é a contribuição diagonal de $G$ dada por

$$
G_{d, k l}(u, v)=G_{k k}(u, u) \delta_{k l} \delta(u, v)
$$

e $G_{n}$ é o resto

$$
G_{n, k l}(u, v)=G_{k l}(u, v)-G_{d, k l}(u, v) .
$$

Nós notamos que pela expansão em polímeros $G$ é analítica no parâmetro de "hopping" $\kappa$. Pelas cotas obtidas no Teorema 3.2 notamos que a série de Neumann de $\Gamma$ é convergente, onde usamos a estimativa de Holmgren ${ }^{1}$ para a norma $\|\bullet\|$ de um operador matricial agindo em $\ell_{2}\left(\mathbb{C}^{4} \times \mathbb{Z}_{o}^{d+1}\right)$. De fato, para mostrarmos a convergência da série (2.11) precisamos mostrar que $\left\|G_{d}^{-1} G_{n}\right\|<1$. Observamos, primeiramente que, $G_{d}^{-1}=1+\mathcal{O}\left(\kappa^{8}\right)$ pelo Teorema 3.1. Vamos considerar, agora, o resto $G_{n}$. Observamos pelo Teorema 3.2 , onde $|x|_{1} \equiv \sum_{i=0}^{d}\left|x^{i}\right|$,

$$
\left|G_{n, k l}(u, v)\right|=\left|G_{k l}(u, v)-G_{d, k l}(u, v)\right| \leq c_{1} \kappa^{2} \delta(u-v)+c_{2} \kappa^{2|u-v|_{1}}(1-\delta(u-v))
$$

e usando o Lema de Holmgren temos

$$
\left|G_{n, k l}\right| \leq\left(\sup _{u} \sum_{v}\left|G_{n, k l}(u, v)\right|\right)^{1 / 2}\left(\sup _{v} \sum_{u}\left|G_{n, k l}(u, v)\right|\right)^{1 / 2} \leq \mathcal{O}\left(\kappa^{2}\right)
$$

Desta forma, $\Gamma$ também é uma função analítica no parâmetro $\kappa$. No método de desacoplamento de hiperplanos nós introduzimos uma variável extra que chamaremos de $\kappa_{p}$ substituindo $\kappa$ para todos os elos, por exemplo, conectando os pontos $x$ e $x+e^{\rho}(\rho=0,1,2,3)$. O ponto aqui é que, podemos controlar a expansão de polímeros de forma que as funções de correlação com diferentes acoplamentos em elos distintos podem ser continuadas analiticamente para $\kappa_{p}$ suficientemente pequeno. A vantagem deste método é que temos um desacoplamento ao longo de um hiperplano inteiro, i.e. para os elos conectando os pontos $x$ e $x+e^{\rho}$. Como função analítica nos novos parâmetros de acoplamento $\kappa_{p}$ podemos, através de estimativas de Cauchy para derivadas obter cotas para o decaimento das funções de correlação envolvidas. Assim

\footnotetext{
${ }^{1}$ Lema de Holmgren: Seja M uma matriz tal que $\sup _{x}\left[\sum_{y} M(x, y)\right] \equiv A$ e $\sup _{y}\left[\sum_{x} M(x, y)\right] \equiv B$ são finitos. Então $M$ define um operador limitado em $\ell^{2}\left(\mathbb{Z}_{o}^{d+1}\right) \operatorname{com}\|M\| \leq A^{1 / 2} B^{1 / 2}$.
} 
mostramos que $\Gamma_{k l}(u, v)$, como função do parâmetro de "hopping" $\kappa$, decai mais rapidamente que $G_{k l}(u, v)$ (Teoremas 3.2 e 4.2). Desta forma, conforme veremos na demonstração do Teorema 5, este decaimento mais rápido implica que a transformada de Fourier $\tilde{\Gamma}_{k l}(p)$ de $\Gamma_{k l}(x=u-v)=\Gamma_{k l}(u, v)$ tem uma região de analiticidade maior em $p^{0}$. Então, como $\tilde{G}(p) \tilde{\Gamma}(p)=I_{4}, \tilde{\Gamma}_{k l}^{-1}(p)=[\operatorname{cof} \tilde{\Gamma}]_{k l}(p) / \operatorname{det}[\tilde{\Gamma}(p)]$ fornece uma extensão meromórfa de $\tilde{G}_{k l}(p)$. O espectro de energia-momento ocorre, para cada $\vec{p}$, como as singularidades de $\tilde{G}_{k l}(p)$ (pelo Corolário 1), ou seja, como pontos dados pelos zeros $\operatorname{det}[\tilde{\Gamma}(p)]$ de $p^{0}$ no eixo $p^{0}$ imaginário. Para analisarmos $\operatorname{det} \tilde{\Gamma}(p)$, não é suficiente obtermos uma cota a longas distâncias para $\Gamma(x)$. Ao contrário, precisamos do seu comportamento preciso para distâncias pequenas, para $|x|_{1} \leq 2$, onde definimos $|x|_{1} \equiv \sum_{i=0}^{d}\left|x^{i}\right|$, para determinar as massas e a abertura ("splitting") de massa até a ordem $\kappa^{4}$.

O comportamento a distâncias curtas e cotas a longas distâncias para $G$ e $\Gamma$ são dados nos próximos dois Teoremas.

Teorema 3 Seja $0<\kappa \lll 1$, c uma constante positiva, $\rho, \sigma=0,1, \ldots, d$, e $e^{0}$ vetor unitário na direção temporal, $e^{i}, e^{j}(i, j=1, \ldots, d)$, os vetores unitários nas direções espaciais, $|\vec{x}|_{1} \equiv$ $\sum_{i=1}^{d}\left|x^{i}\right|$ e $\epsilon, \epsilon^{\prime}= \pm 1$. As seguintes propriedades valem para $G$ :

1.

$$
G_{k l}(x)=\left\{\begin{array}{lll}
\delta_{k l}+\mathcal{O}\left(\kappa^{8}\right) & , \quad x=0 ; \\
\delta_{k l} \kappa^{2}+\mathcal{O}\left(\kappa^{6}\right) & , \quad x=\epsilon e^{0} \\
c_{2} \delta_{k l} \kappa^{2}+\mathcal{O}\left(\kappa^{6}\right) & , \quad x=\epsilon e^{j} \\
\delta_{k l} \kappa^{4}+\mathcal{O}\left(\kappa^{8}\right) & , \quad x=2 \epsilon e^{0} \\
c_{2} \delta_{k l} \kappa^{4}+\mathcal{O}\left(\kappa^{8}\right) & , \quad x=2 \epsilon e^{j} \\
c_{k l}(x) \kappa^{4}+\mathcal{O}\left(\kappa^{8}\right) & , \quad x=\epsilon e^{\rho}+\epsilon^{\prime} e^{\sigma}, \rho<\sigma .
\end{array}\right.
$$

as constantes independentes de $\kappa$ são dadas por $c_{2}=1 / 4$, e

$$
c_{k l}(x)=\left\{\begin{array}{l}
\delta_{k l} / 2, x=\epsilon e^{0}+\epsilon^{\prime} e^{\rho} \\
c_{k l}^{i j}, x=\epsilon e^{i}+\epsilon^{\prime} e^{j}, i<j
\end{array},\right.
$$

onde $c_{k l}^{i j}$ é dado por: $c_{k l}^{12}$ é diagonal com $4 c_{11}^{12}=4 c_{44}^{12}=1$ e $c_{22}^{12}=c_{33}^{12}=0,4 c_{11}^{13}=8 c_{22}^{13}=$ $8 c_{33}^{13}=-8 c_{23}^{13}=-8 c_{32}^{13}=1,4 c_{11}^{23}=8 c_{22}^{23}=8 c_{33}^{23}=8 c_{23}^{23}=8 c_{32}^{23}=1$, e todos os elementos não mencionados até aqui são nulos.

2.

$$
\left|G_{k l}(x)\right| \leq c|\kappa|^{2\left|x^{0}\right|+2|\vec{x}|_{1}}
$$

o que implica na analiticidade de $\tilde{G}_{k l}\left(p^{0}, \overrightarrow{0}\right)$ na região $\left|\operatorname{Im} p^{0}\right|<-(2-\epsilon) \ln \kappa$.

3. $G_{k l}(x)=d_{k} G_{k l}\left(x^{\prime}\right) d_{l}^{*}$, onde 
(a) para $x^{\prime}=\left(x^{0},-x^{2}, x^{1}, x^{3}\right), d_{1}=1, d_{2}=-i, d_{3}=i, d_{4}=1$;

(b) para $x^{\prime}=\left(x^{0},-x^{1},-x^{2}, x^{3}\right), d_{1}=1, d_{2}=-1, d_{3}=-1, d_{4}=1$;

(c) para $x^{\prime}=\left(x^{0},-x^{1},-x^{2},-x^{3}\right), d_{k}=-1$, para $k=1,2,3,4$.

Também,

$$
G\left(e^{1}+e^{2}\right)=S G\left(e^{1}+e^{3}\right) S^{\dagger}
$$

onde $S=S^{t}$ e $S^{-1}=S^{\dagger}$ (superscrito $t$ significa transposta e $\dagger$ conjugação Hermitiana). $S$ tem os elementos de matrix $S_{11}=1, S_{12}=S_{13}=S_{14}=S_{44}=0, S_{22}=S_{33}=S_{23}=$ $1 / 2$ e $S_{24}=S_{34}^{*}=i \sqrt{2} / 2$.

Observação 3 A igualdade dos elementos elementos na diagonal de $c_{k l}^{13}$ e $c_{k l}^{23}$ segue dos resultados de simetria do Teorema 1 , ou seja, rotações de $\pi / 2$ em relação ao eixo $e^{3}$.

Observação 4 O terceiro resultado acima será usado para simplificar a prova do Teorema 4 , já que, relaciona a função de dois pontos em pontos distintos da rede $\mathbb{Z}_{o}^{d}$. Conforme discutimos precisamos do comportamento de $G(x)$ para distâncias $|x|_{1} \leq 2$. Desta forma, usando as simetrias do Teorema 1 é necessário determinar $G_{k l}(x)$ para os pontos $x=0, e^{0}, e^{1}, 2 e^{1}, e^{1}+e^{3}$.

O comportamento a pequenas distâncias e o decaimento de $\Gamma(x)$ são dados por:

Teorema 4 Considerando as hipóteses do Teorema 3, com $c_{2}$ e $c_{k l}(x)$ lá definidos, temos:

1.

$$
\Gamma_{k l}(x)=\left\{\begin{array}{l}
\delta_{k l}+\left(2+2 d c_{2}^{2}\right) \delta_{k l} \kappa^{4}+\mathcal{O}\left(\kappa^{8}\right), x=0 ; \\
-\delta_{k l} \kappa^{2}+\mathcal{O}\left(\kappa^{8}\right), x=\epsilon e^{0} ; \\
-c_{2} \delta_{k l} \kappa^{2}+\mathcal{O}\left(\kappa^{6}\right), x=\epsilon e^{j} ; \\
\mathcal{O}\left(\kappa^{10}\right), x=2 \epsilon e^{0} ; \\
\left(-c_{2}+c_{2}^{2}\right) \delta_{k l} \kappa^{4}+\mathcal{O}\left(\kappa^{8}\right), x=2 \epsilon e^{j} ; \\
{\left[-c_{k l}(x)+2 c_{2}^{2} \delta_{k l}\right]\left(1-\delta_{0 \rho}\right)\left(1-\delta_{0 \sigma}\right) \kappa^{4}+\mathcal{O}\left(\kappa^{8}\right),} \\
\mathcal{O}\left(\kappa^{8}\right),\left|x^{0}\right|=1,|\vec{x}|_{1}=2 ; \\
\mathcal{O}\left(\kappa^{10}\right),\left|x^{0}\right|=2,|\vec{x}|_{1}=1 ; \\
\mathcal{O}\left(\kappa^{12}\right),\left|x^{0}\right|=3,|\vec{x}|_{1}=0 .
\end{array}\right.
$$

2.

$$
\left|\Gamma_{k l}(x)\right| \leq\left\{\begin{array}{l}
c|\kappa|^{2+4\left(\left|x^{0}\right|-1\right)+2|\vec{x}|_{1}},\left|x^{0}\right|>1 \\
c|\kappa|^{2\left|x^{0}\right|+2|\vec{x}|_{1}},\left|x^{0}\right| \leq 1
\end{array}\right.
$$

o que implica na analiticidade de $\tilde{\Gamma}_{k l}\left(p^{0}, \overrightarrow{0}\right)$ na região $\left|\operatorname{Im} p^{0}\right|<-(4-\epsilon) \ln \kappa$. 
Considerando o Teorema 4.1 notamos que para $\left|x^{0}\right|=1,|\vec{x}|_{1}=2 ;\left|x^{0}\right|=2,|\vec{x}|_{1}=1$; $\left|x^{0}\right|=3,|\vec{x}|_{1}=0$ nós esperaríamos as contribuições, $\mathcal{O}\left(\kappa^{6}\right) ; \mathcal{O}\left(\kappa^{8}\right) ; \mathcal{O}\left(\kappa^{10}\right)$ respectivamente devido ao decaimento de $\left|\Gamma_{k l}(x)\right| \leq c|\kappa|^{2+4\left(\left|x^{0}\right|-1\right)+2|\vec{x}|_{1}}$ do Teorema 4.2. A ausência de termos de ordem inferior em $\Gamma_{k l}$, comparada com $G_{k l}$, para $\left|x^{0}\right|=1,2,3$, é devido a cancelamentos explícitos na série de Neumann e aprimora as cotas obtidas pelo método de desacoplamento de hiperplanos.

Nós determinamos o espectro de massa até $\mathcal{O}\left(\kappa^{4}\right)$ pelos valores de $\Gamma_{k l}(x)$ até a distância $|x|_{1} \leq 2$, e $k=l$. A contribuição de ordem $\kappa^{4}$ para $\Gamma(x)$, para estes valores de $x$, vem do segundo e terceiros termos em $G_{n}$ (veja eq. (2.13)) na série de Neumann da eq. (2.11). Os elementos de segunda ordem de $k$ já que são produtos de dois termos de ordem $\kappa^{2}$ de $G_{n, k l}(x)$, para pontos $x$ de distância um, que são diagonais e independentes do índice de spin $k$. Para o termo de primeira ordem em $G_{n}(x),|x|_{1}=2$, as contribuições de ordem $\kappa^{4}$ vêm de contribuições lineares e angulares. Contribuições lineares têm dois conjuntos de elos sobrepostos, com orientação oposta, conectando, por exemplo, os pontos 0 a $\epsilon e^{\rho}$ e daí até $2 \epsilon e^{\rho}$; e as propriedades das matrizes $\Gamma$ da eq. (2.27) guarantem que estas contribuições comportam como o termo de ordem $\kappa^{2}$ devido à conservação de spin, diagonal e independente de $k$ em $G_{n, k l}(x),|x|_{1}=1$, e não estão associados à abertura de massa também. Esquematicamente temos, com $x_{1}=0, x_{2}=\epsilon e^{\rho}$ e $x_{3}=2 \epsilon e^{\rho}$ :

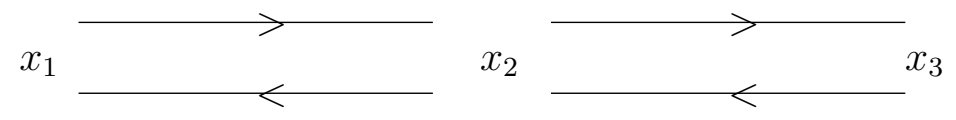

Contribuições angulares para $G_{n, k l}$ são dadas por pontos da forma $x=\epsilon e^{i}+\epsilon^{\prime} e^{j}$, $i, j=1,2, \ldots, d, i<j, \epsilon, \epsilon^{\prime}= \pm 1$. Estas contribuições são geometricamente do tipo L e estão associadas a dois conjuntos de dois elos, com orientações opostas; um conjunto conectando os pontos $0 \rightarrow \epsilon e^{i}$ e o outro conectando $\epsilon e^{i} \rightarrow \epsilon e^{i}+\epsilon^{\prime} e^{j}$, ou um conjunto conectando os pontos $0 \rightarrow \epsilon^{\prime} e^{j}$ e o outro conectando $\epsilon^{\prime} e^{j} \rightarrow \epsilon e^{i}+\epsilon^{\prime} e^{j}$. Esquematicamente temos, com $x_{1}=0$, $x_{2}=\epsilon e^{i}, x_{4}=\epsilon^{\prime} e^{j}$ e $x_{3}=\epsilon e^{i}+\epsilon^{\prime} e^{j}$ : 

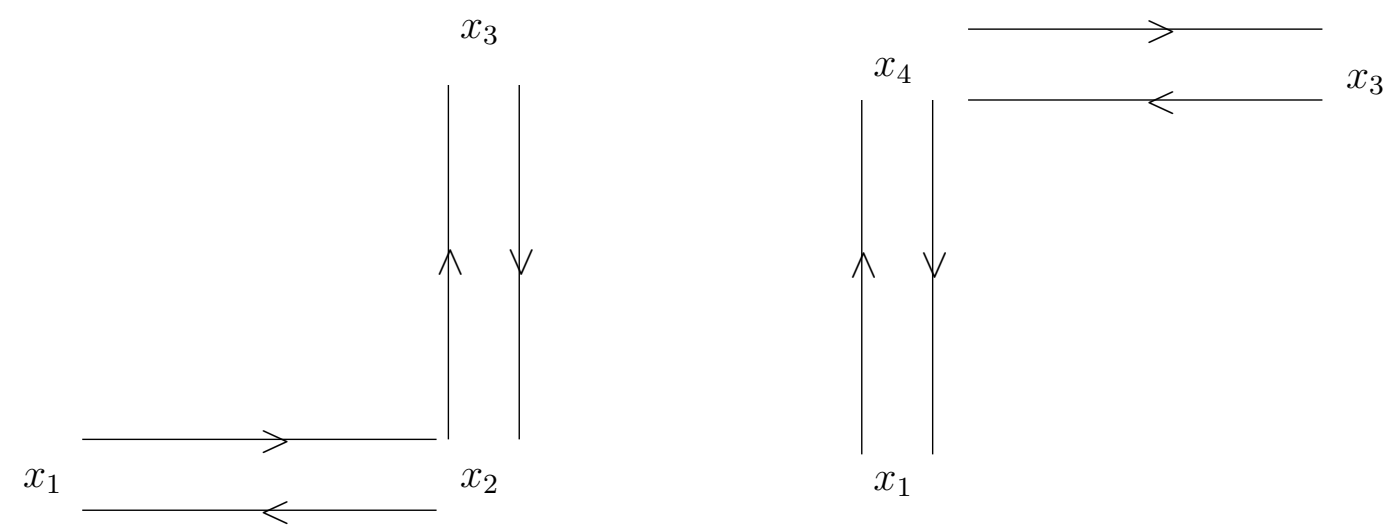

Conforme veremos na Seção 2.3, as configurações do tipo $L$ contribuem para a abertura de massa na $\mathcal{O}\left(\kappa^{4}\right)$ para $d=2,3$.

Antes de mencionarmos nossos resultados a respeito do espectro de massas e curvas de dispersão, daremos uma idéia do comportamento assintótico da massa para $\kappa \rightarrow 0$. Usando somente a contribuição diagonal no índice de spin de $\Gamma_{k l}(x), x=0$ e $x=(1, \overrightarrow{0})$, a equação para a massa $m$ é

$$
\operatorname{det} \tilde{\Gamma}\left(p^{0}=i m, \vec{p}=\overrightarrow{0}\right) \approx\left(1-\kappa^{2} e^{m}\right)^{4}=0, m \in \mathbb{R}
$$

desta forma temos uma massa $m$ com magnitude $-2 \ln \kappa$, singular em $\kappa=0$, e degenerescência de quarta ordem.

Para determinar o espectro de energia-momento, usamos simetrias do Teorema 1 para $\vec{p}=\overrightarrow{0}$. Conforme veremos $\tilde{\Gamma}\left(p^{0}=i m, \vec{p}=\overrightarrow{0}\right)$ é diagonal. A determinação da parte não-singular da massa pode ser formulada como um problema envolvendo o Teorema da função implícita. Para $\vec{p} \neq \overrightarrow{0}$, não conseguimos mostrar que $\tilde{\Gamma}\left(p^{0}=i m, \vec{p}\right)$ é diagonal, mas a forma assintótica (para $\kappa$ pequeno) das curvas de dispersão podem ser obtidas usando o Teorema de Rouché ${ }^{2}$ para os zeros de $\operatorname{det} \tilde{\Gamma}\left(p^{0}=i w(\vec{p}), \vec{p}\right)$. Também, nossos resultados em $\mathcal{H}_{m}$ são estendidos para todo subespaço $\mathcal{H}_{e}$ usando o método de subtrações Euclideanas de [31]. Mais detalhes serão dados adiante.

Os resultados para o espectro de energia-momento para uma partícula mesônica são dados no Teorema abaixo.

Teorema 5 Considerando as hipóteses do Teorema 3, os seguintes resultados espectrais valem no subespaço par $\mathcal{H}_{e}$ do espaço de Hilbert físico $\mathcal{H}$.

\footnotetext{
${ }^{2}$ Teorema de Rouché: Sejam $f$ e $g$ holomorfas dentro de um contorno $\gamma$ e suponha que $|f(z)|>|g(z)|$ em $\gamma \cup \partial \gamma$. Então, $f$ e $f+g$ têm o mesmo número de zeros dentro de $\gamma$.
} 
1. Para qualquer ordem em $\kappa$ e para $d=2,3$, o espectro de massa em $\mathcal{H}_{e}$ e no intervalo de energia $(0,-(4-\epsilon) \ln \kappa), \epsilon>0$, consiste em três massas distintas dadas por $\operatorname{diag}[(a+$ $\left.c)^{-1}, b^{-1}, b^{-1},(a-c)^{-1}\right], a \equiv \tilde{G}_{11}\left(p^{0}, \overrightarrow{0}\right), b \equiv \tilde{G}_{33}\left(p^{0}, \overrightarrow{0}\right)$ e $c \equiv \tilde{G}_{44}\left(p^{0}, \overrightarrow{0}\right)$. Até, e incluindo, a ordem $\kappa^{4}, a-c=b$, para $d=3$, e $c=0$, para $d=2$, e existem somente duas massas distintas $m_{a}$ e $m_{b}$ dadas por

$$
m_{j}=-2 \ln \kappa+r_{j}(\kappa), \quad j=a, b,
$$

onde $r_{j}(\kappa) \equiv \sum_{n=2}^{\infty} b_{j, n} \kappa^{n}$ é real e analítica em $\kappa$ na vizinhança do ponto 0 , para cada $d=2$, 3. Nós obtemos, com as constantes dadas no Teorema 3,

$$
\begin{aligned}
r_{c}(\kappa)= & -2 d c_{2} \kappa^{2}+\left[4\left(c_{k k}^{12}+c_{k k}^{13 ; 23}\right)\right. \\
& \left.-\left(1-2 d c_{2}-24 c_{2}^{2}+20 d c_{2}^{2}\right)-2 d^{2} c_{2}^{2}\right] \kappa^{4}+\mathcal{O}\left(\kappa^{6}\right),
\end{aligned}
$$

onde $c_{k k}^{13 ; 23} \equiv c_{k k}^{13}+c_{k k}^{23}$ deve ser omitida no caso $d=2$. De novo, até e incluindo a ordem $\kappa^{4}$, para $d=3$, a massa $m_{b}$ está associada com $k=1$ e tem multiplicidade um; $m_{a}$ está associada com $k=2,3,4$, e tem multiplicidade três. A abertura ("splitting") de massa é dada por $m_{a}-m_{b}=2 \kappa^{4}+\mathcal{O}\left(\kappa^{6}\right)$. Para $d=2,\left(m_{b}\right) m_{a}$ está associada com $k=1,4 \quad(k=2,3)$, e ambas têm multiplicidade dois. A abertura de massa é dada por $m_{a}-m_{b}=\kappa^{4}+\mathcal{O}\left(\kappa^{6}\right)$.

2. O espectro de energia-momento em $\mathcal{H}_{e}$ e no intervalo de energia $(0,-(4-\epsilon) \ln \kappa), \epsilon>0$, consiste de quatro curvas de dispersão (não necessariamente distintas), cada uma das quais tem a forma

$$
w(\vec{p})=-2 \ln \kappa-2 d c_{2} \kappa^{2}+c_{2} \kappa^{2} \sum_{j=1}^{d} 2\left(1-\cos p^{j}\right)+\mathcal{O}\left(\kappa^{4}\right) .
$$

As curvas $w(\vec{p})$ são funções crescentes em cada uma de suas componentes $p^{j}$ de $\vec{p}$, e são convexas para $|\vec{p}|$ pequeno.

Observação $5 \mathrm{Em}$ contraste com a eq. (2.20), para férmions livres e $|\vec{p}|$ pequeno, o coeficiente do termo $|\vec{p}|^{2}$ em $w(\vec{p})$ é proporcional a $\kappa$.

Observação 6 A ação da conjugação de carga definida no Teorema 1 deixa o espaço $\mathcal{H}_{m}$ estável, então temos a mesma representação espectral para partículas e anti-partículas, dada pela eq. (2.9). Com relação às funções de correlação de mésons, a função de dois mésons para a anti-partícula (que chamamos de $G^{\prime}$ ) está relacionada com $G$ por $G^{\prime}=T G T^{-1}$, com $T=\operatorname{diag}[1,-1,-1,-1]$, para $d=3$. Para $d=2$, $T$ deve ser substituído por $U$, onde $U=\operatorname{diag}[-1,-1,-1,1]$. Como a massa e as curvas de dispersão são determinadas pela 
equação implícita $\operatorname{det} \tilde{\Gamma}\left(p_{0}, \vec{p}\right)=0$, o espectro de massa e as curvas de dispersão das partículas e anti-partículas são idênticas.

Resultados espectrais para bárions foram provados em $[12,11]$ e aqui nos limitamos a enunciar o Teorema a respeito da existência de partículas bariônicas em $(2+1)$ e $(3+1)$ dimensões, um sabor e usando matrizes de Dirac $4 \times 4$.

Os campos bariônicos são definidos em $\mathcal{H}_{o} \subset \mathcal{H}$ e estão associados a variáveis de Grassmann invariantes por transformaçoẽs de calibre dadas por $\phi_{s}(u)$ e $\bar{\phi}_{s}(v)$, onde $s$ refere-se ao índice de spin, $\operatorname{com} \epsilon_{a b c}$ denotando o símbolo de Levi-Civita:

$$
\phi_{s}(u)=\left\{\begin{array}{lll}
\frac{1}{6} \epsilon_{a b c} \psi_{1, a}(u) \psi_{1, b}(u) \psi_{1, c}(u) & , & s=3 / 2 \\
\frac{1}{2 \sqrt{3}} \epsilon_{a b c} \psi_{1, a}(u) \psi_{1, b}(u) \psi_{2, c}(u) & , & s=1 / 2 \\
\frac{1}{2 \sqrt{3}} \epsilon_{a b c} \psi_{1, a}(u) \psi_{2, b}(u) \psi_{2, c}(u) & , & s=-1 / 2 \\
\frac{1}{6} \epsilon_{a b c} \psi_{2, a}(u) \psi_{2, b}(u) \psi_{2, c}(u) & , & s=-3 / 2 ;
\end{array}\right.
$$

e

$$
\bar{\phi}_{s}(v)=\left\{\begin{array}{lll}
\frac{1}{6} \epsilon_{a b c} \bar{\psi}_{1, a}(v) \bar{\psi}_{1, b}(v) \bar{\psi}_{1, c}(v) & , & s=3 / 2 \\
\frac{1}{2 \sqrt{3}} \epsilon_{a b c} \bar{\psi}_{1, a}(v) \bar{\psi}_{1, b}(v) \bar{\psi}_{2, c}(v) & , & s=1 / 2 \\
\frac{1}{2 \sqrt{3}} \epsilon_{a b c} \bar{\psi}_{1, a}(v) \bar{\psi}_{2, b}(v) \bar{\psi}_{2, c}(v) & , & s=1 / 2 \\
\frac{1}{6} \epsilon_{a b c} \bar{\psi}_{2, a}(v) \bar{\psi}_{2, b}(v) \bar{\psi}_{2, c}(v) & , & s=-1 / 2 .
\end{array}\right.
$$

Agora apresentamos os resultados a respeito da existência de partículas bariônicas obtidos [12].

Teorema 6 Com as mesmas hipóteses do Teorema 3, considerando o espectro de energiamomento para um bárion temos:

1. O espectro de massa em $\mathcal{H}_{o}$ e no intervalo de energia $(0,-(5-\epsilon) \ln \kappa), \epsilon>0$, contém duas massas (não necessariamente distintas) $M_{3 / 2}=M_{-3 / 2}$ e $M_{1 / 2}=M_{-1 / 2}$, cada uma com multiplicidade dois (partículas bariônicas e suas anti-partículas) e

$$
M_{s}=-3 \ln \kappa+r_{s}(\kappa),
$$

onde $r_{s}(\kappa) \equiv r_{s}(\kappa, d)$ é real e analítica em $\kappa$, para cada $d$. Nós obtemos, com $c_{3}=-3 / 4$, $c_{6}=-9 / 2, c_{30}=-6, c_{s 12}=-(9 / 4)\left[-\delta_{|s| 3 / 2}+\delta_{|s| 1 / 2}\right]$ e $c_{s 13}=c_{s 23}=-(9 / 8)\left[\delta_{|s| 3 / 2}-\right.$ $\left.\delta_{|s| 1 / 2}\right]$

$$
\begin{aligned}
r_{s}(\kappa)= & -\ln \left|c_{30}\right|+2 d c_{3} \kappa^{3}+\left[4 d c_{3}^{2}+2 d c_{6}+c_{30}^{2}\right. \\
& \left.-\frac{1}{2}\left(2 d c_{3}\right)^{2}+4\left(c_{s 12}+c_{s 13}+c_{s 23}\right)\right] \kappa^{6}+\mathcal{O}\left(\kappa^{7}\right),
\end{aligned}
$$


onde $c_{s 13}$ e $c_{s 23}$ devem ser omitidos para $d=2$. Para $d=3, c_{s 12}+c_{s 13}+c_{s 23}=0$, então não existe abertura de massa até e incluindo $\mathcal{O}\left(\kappa^{6}\right)$. Para $d=2$, a abertura de massa é dada por $M_{3 / 2}-M_{1 / 2}=4\left(c_{3 / 2} 12-c_{1 / 2} 12\right) \kappa^{6}+\mathcal{O}\left(\kappa^{7}\right)=18 \kappa^{6}+\mathcal{O}\left(\kappa^{7}\right)$.

2. O espectro de energia-momento em $\mathcal{H}_{o}$ e no intervalo de energia $(0,-(5-\epsilon) \ln \kappa), \epsilon>0$, consiste de quatro curvas de dispersão, duas a duas idênticas e não necessariamente distintas entre si, cada uma das quais tem a forma

$$
w(\vec{p})=-3 \ln \kappa-\ln \left|c_{30}\right|+2 d c_{3} \kappa^{3}-c_{3} \kappa^{3} \sum_{j=1}^{d} 2\left(1-\cos p^{j}\right)+\mathcal{O}\left(\kappa^{6}\right) .
$$

As curvas $w(\vec{p})$ são funções crescentes em cada uma de suas componentes $p^{j}$ de $\vec{p}$, e são convexas para $|\vec{p}|$ pequeno.

\subsection{Cotas para o decaimento e comportamento a pequenas distâncias da função de dois mésons $G$ e sua inversa por convolução $\Gamma$}

Nesta seção, apresentamos uma prova da Proposição 1 e dos Teoremas 3 e 4 que descrevem o comportamento de $G$ e $\Gamma$ em função do parâmetro de "hopping" $\kappa$. Nós começamos esta seção dando uma prova da Proposição 1.

Prova da Proposição 1: Pela fórmula de F-K da eq. (1.6) temos a seguinte relação

$$
\left(M(1 / 2, \overrightarrow{0}), \check{T}_{0}^{x^{0}-1} \check{T}^{\vec{x}} L(1 / 2, \overrightarrow{0})\right)_{\mathcal{H}}=\left\langle\left[T_{0}^{x^{0}-1} T^{\vec{x}} L(1 / 2, \overrightarrow{0})\right] \Theta M(1 / 2, \overrightarrow{0})\right\rangle
$$

Fazendo $x^{0}=v^{0}-u^{0}>0$ temos, pela fórmula de F-K

$$
\begin{aligned}
\left(M(1 / 2, \overrightarrow{0}), \check{T}_{0}^{v^{0}-u^{0}-1} \check{T}^{\vec{x}} L(1 / 2, \overrightarrow{0})\right)_{\mathcal{H}} & =\left\langle\left[T_{0}^{v^{0}-u^{0}-1} T^{\vec{x}} L(1 / 2, \overrightarrow{0})\right](\Theta M)(-1 / 2, \overrightarrow{0})\right\rangle \\
& =\left\langle\left[T_{0}^{v^{0}-\frac{1}{2}} T^{\vec{x}}(1 / 2, \overrightarrow{0})\right] T_{0}^{u^{0}+\frac{1}{2}}(\Theta M)(-1 / 2, \overrightarrow{0})\right\rangle,
\end{aligned}
$$

onde para obtermos a segunda igualdade acima usamos a invariância das funções de correlação por translação temporal. Agora definimos

$$
L\left(v^{0}, \vec{x}\right) \equiv T_{0}^{v^{0}-\frac{1}{2}} L(1 / 2, \overrightarrow{0}) \text { e }(\Theta M)\left(u^{0}\right) \equiv T_{0}^{u^{0}+\frac{1}{2}}(\Theta M)(-1 / 2, \overrightarrow{0}) .
$$

Desta forma $L\left(v^{0}, \vec{x}\right)$ tem suporte em $v^{0}$ e $(\Theta M)\left(u^{0}\right)$ em $u^{0}$. A fórmula de F-K fornece

$$
\left(M, \check{T}_{0}^{x^{0}-1} \check{T}^{\vec{x}} L\right)_{\mathcal{H}}= \pm\left\langle(\Theta M)\left(u^{0}\right) L\left(v^{0}, \vec{x}\right)\right\rangle, \quad u^{0}<v^{0}
$$


com o sinal - se ambos os campos compostos $M$ e $L$ possuem um número ímpar de campos fundamentais $(\psi$ ou $\bar{\psi})$, i.e. se pertencem a $\mathcal{H}_{o}$, e o sinal + caso contrário.

Por outro lado temos para $u_{0}>v_{0}$

$$
\begin{aligned}
\left(M(1 / 2, \overrightarrow{0}), \check{T}_{0}^{u^{0}-v^{0}-1} \check{T^{\vec{x}}} L(1 / 2, \overrightarrow{0})\right)_{\mathcal{H}} & =\left(\check{T}_{0}^{u^{0}-v^{0}-1} M(1 / 2, \overrightarrow{0}), L(1 / 2, \vec{x})\right)_{\mathcal{H}} \\
& =\left(L(1 / 2, \vec{x}), \check{T}_{0}^{u^{0}-v^{0}-1} M(1 / 2, \overrightarrow{0})\right)_{\mathcal{H}} \\
& =\left\langle\left[T_{0}^{u^{0}-v^{0}-1} T^{\vec{x}} M(1 / 2, \overrightarrow{0})\right](\Theta L)(-1 / 2, \vec{x})\right\rangle^{*} \\
& =\left\langle M\left(u^{0}\right)(\Theta L)\left(v^{0}, \vec{x}\right)\right\rangle^{*},
\end{aligned}
$$

onde para obtermos a primeira igualdade usamos o fato que $\check{T}_{0}$ é auto-adjunto, a segunda igualdade segue das propriedades do produto interno. A terceira igualdade segue usando a fórmula de F-K e para obtermos a quarta igualdade usamos invariância das funções de correlação por translações no tempo.

As eqs. (4.27) e (4.28) motivam a definição da função de correlação, com $x^{0}=v^{0}-u^{0}$, $x=\left(x^{0}, \vec{x}\right)$

$$
\mathcal{G}_{M, L}(x) \equiv \begin{cases} \pm\left\langle(\Theta M)\left(u^{0}\right) L\left(v^{0}, \vec{x}\right)\right\rangle, & u^{0} \leq v^{0} \\ \left\langle M\left(u^{0}\right)(\Theta L)\left(v^{0}, \vec{x}\right)\right\rangle^{*}, & u^{0}>v^{0}\end{cases}
$$

A definição para $u^{0}=v^{0}$ é obtida estendendo a definição de $u^{0}<v^{0}$. Para a representação espectral temos, $x^{0} \neq 0$

$$
\mathcal{G}_{M, L}(x)=\int_{-1}^{1} \int_{T^{d}} \lambda_{0}^{\left|x^{0}\right|-1} e^{i \vec{\lambda} \cdot \vec{x}} d\left(M(1 / 2), \mathcal{E}\left(\lambda_{0}, \vec{\lambda}\right) L(1 / 2)\right)_{\mathcal{H}}
$$

Agora usamos o método de desacoplamento de hiperplanos para obter cotas para $G$ e $\Gamma$, de acordo com o Teorema 3 .

Ao longo deste trabalho encontramos integrais sobre campos de calibre dos elementos de matriz $g_{i j}(i, j=1,2,3)$, (onde $g_{i j} \in S U(3)$ e suprimimos da notação pontos da rede) e suas inversas $g_{i j}^{-1}$. Creutz desenvolveu um método para o cálculo de integrais de calibre baseado num funcional gerador. No Apêndice A, mostramos explicitamente o procedimento usado por Creutz para avaliar integrais de calibre. Repetimos o cálculo explícito das integrais de dois, três e quatro campos de calibre usadas ao longo deste trabalho.

Nós também usaremos as seguintes propriedades envolvendo as matrizes $\Gamma(\rho, \sigma=$ $0,1,2,3$, and $\left.\epsilon, \epsilon^{\prime}= \pm 1\right)$

$$
\begin{aligned}
\Gamma^{\epsilon e^{\rho}} \Gamma^{-\epsilon e^{\rho}} & =0, \\
\Gamma^{\epsilon e^{\rho}} \Gamma^{\epsilon e^{\rho}} & =-2 \Gamma^{\epsilon e^{\rho}}, \\
\Gamma^{\epsilon e^{\rho}} \Gamma^{\epsilon^{\prime} e^{\sigma}} & =2 I_{4}-\Gamma^{-\epsilon^{\prime} e^{\sigma}} \Gamma^{-\epsilon e^{\rho}} .
\end{aligned}
$$


Notamos que usando matrizes de spin $2 \times 2$ fórmulas similares são válidas com as devidas adaptações, i.e. $I_{4} \mapsto I_{2}$.

Vamos agora nos concentrar na obtenção de propriedades de decaimento para as funções de correlação truncadas definidas de uma forma geral por

$$
\langle F(u) ; H(v)\rangle_{T}=\left\langle\left[T_{0}^{u^{0}-1 / 2} T^{\vec{u}} F(1 / 2, \overrightarrow{0})\right] ;\left[T_{0}^{v^{0}-1 / 2} T^{\vec{v}} H(1 / 2, \overrightarrow{0})\right]\right\rangle_{T}
$$

onde $F, H \in \mathcal{H}_{e}, T_{0}$ é a translação por $e^{0}$ e $T^{\vec{u}}=T_{1}^{u^{1}} \ldots T_{d}^{u^{d}}$ é a translação no espaço por $\vec{u}=\left(u^{1}, \ldots, u^{d}\right) \in \mathbb{Z}^{d}$.

No método de desacoplamento de hiperplanos para $u^{0}<v^{0}, p \in \mathbb{Z}, u^{0}+1 / 2 \leq p \leq$ $v^{0}-1 / 2$, substituímos o parâmetro de "hopping" $\kappa>0$ multiplicando a parte não-local que acopla férmions e campos de calibre na ação (1.2) (não o $\kappa$ em $M$ ) por $\kappa_{p} \in \mathbb{C}$ e denotando $\partial^{r} / \partial \kappa_{p}^{r}$ por $\partial^{r}$ e por $\partial_{0}^{r}$ seu valor em $\kappa_{p}=0$, as seguintes propriedades seguem. Nós notamos que, para mostrarmos o Lema 1, devemos levar em consideração o complexo conjugado que aparece na definição da função de dois da eq. (2.6) para o ordenamento temporal $u^{0}>v^{0}$. Desta forma, se $u^{0}>v^{0}, v^{0}+1 / 2 \leq p \leq u^{0}-1 / 2$, após tomarmos * nas funções de correlação substituímos $\kappa$ por $\kappa_{p}$ de tal forma que estas funções dependam somente de $\kappa_{p}$ e não $\kappa_{p}^{*}$.

Lema 1 Considerando as derivadas de $G$, temos:

1. Se $u^{0} \neq v^{0}, \partial_{0}^{r}\langle F(u) ; H(v)\rangle_{T}=0, r=0,1,3$.

2. Se $u^{0}<v^{0}$, e $r=2$,

$$
\partial_{0}^{2}\langle F(u) ; H(v)\rangle_{T}=\left.2 \sum_{w \mid w^{0}=-1 / 2+p}\left\langle F(u) \Pi_{k}(w)\right\rangle\left\langle\mu_{k}\left(w+e^{0}\right) H(v)\right\rangle\right|_{\kappa_{p}=0}
$$

3. Se $u^{0}>v^{0}$, e $r=2$,

$$
\partial_{0}^{2}\langle F(u) ; H(v)\rangle_{T}^{*}=\left.2 \sum_{w \mid w^{0}=-1 / 2+p}\left\langle F(u) \mu_{k}\left(w+e^{0}\right)\right\rangle^{*}\left\langle\Pi_{k}(w) H(v)\right\rangle^{*}\right|_{\kappa_{p}=0} .
$$

Prova do Lema 1: A prova deste Lema está dada no Apêndice B (ver eq. (B.14)).

Para calcular as derivadas de $\Gamma$ nas variáveis $\kappa_{p}$, usamos a fórmula de Leibniz para a derivada do produto $\Gamma G$ e observando que $\Gamma G=1$, obtemos

$$
\partial^{r} \Gamma=-\sum_{s=0}^{r-1}\left(\begin{array}{l}
r \\
s
\end{array}\right) \Gamma \partial^{r-s} G \partial^{s} \Gamma .
$$

As primeiras três derivadas de $\Gamma$ em $\kappa_{p}=0$ são dadas no próximo Lema.

Lema 2 Para as derivadas de $\Gamma$, temos: 
1. Se $u^{0} \neq v^{0}, \partial_{0}^{r} \Gamma(u, v)=0, r=0,1$.

2. Se $u^{0}<v^{0}, \partial_{0}^{2} \Gamma(u, v)=-2 \sum_{w \mid w^{0}=-1 / 2+p} \delta(u, w) \delta\left(w+e^{0}, v\right)$.

3. Se $u^{0}>v^{0}, \partial_{0}^{2} \Gamma(u, v)=-2 \sum_{w \mid w^{0}=-1 / 2+p} \delta\left(u, w+e^{0}\right) \delta(w, v)$.

4. Se $\left|u^{0}-v^{0}\right|>1, \partial_{0}^{3} \Gamma(u, v)=0$.

Prova do Lema 2: Neste Lema apresentamos a prova dos dois primeiros ítems para o ordenamento temporal $u^{0}<v^{0}$. O terceiro item e o ordenamento temporal $u^{0}<v^{0}$ é obtido de forma similar.

Para a primeira afirmação temos, considerando, $\left.\Gamma(u, v)\right|_{\kappa_{p}=0}=0$ que o espaço $\ell_{2}\left(\mathbb{Z}_{o}^{d+1}\right)$ $(d=1,2)$ quebra em uma soma direta $\ell^{2}\left(A_{\leq}\right) \oplus \ell^{2}\left(A_{>}\right)$onde $A_{\leq}=\left\{u \in \mathbb{Z}_{o}^{d+1} \mid u^{0} \leq p-1 / 2\right\}$ e $A_{\leq}=\left\{u \in \mathbb{Z}_{o}^{d+1} \mid u^{0} \geq p+1 / 2\right\}$ de tal forma que $G_{A_{\leq}} \oplus G_{A_{>}}$e desta forma $\Gamma_{A_{\leq}} \oplus \Gamma_{A_{>}}$.

Considerando $\partial_{0} \Gamma$ temos, pela eq. (2.30),

$$
\partial_{0} \Gamma=-\left.\Gamma \partial G \Gamma\right|_{\kappa_{p}=0},
$$

ou em termos de núcleos

$$
\begin{aligned}
\left.\Gamma \partial G \Gamma(u, v)\right|_{\kappa_{p}=0} & =\left.\sum_{w, z} \Gamma(u, w) \partial G(w, z) \Gamma(z, v)\right|_{\kappa_{p}=0} \\
& =\left.\sum_{w, z \mid w^{0}+1 / 2 \leq p \leq z^{0}-1 / 2} \Gamma(u, w) \partial G(w, z) \Gamma(z, v)\right|_{\kappa_{p}=0}=0 .
\end{aligned}
$$

Para obtermos a segunda igualdade na eq. (2.31) usamos, a primeira parte do Lema 2.1, i.e. $\left.\Gamma(u, v)\right|_{\kappa_{p}=0}=0$ se $u^{0} \neq v^{0}$, em particular, $\Gamma(u, w)=0$ se $w^{0}+1 / 2 \geq p$ e $\Gamma(z, v)=0$ se $z^{0} \geq p-1 / 2$. Para obtermos a terceira igualdade lembramos do Lema 1.1 que como $w^{0}+1 / 2 \leq p \leq z^{0}-1 / 2$ temos, $w^{0} \leq p-1 / 2$ e $z^{0} \geq p+1 / 2$, e daí segue que $w^{0} \neq z^{0}$ que por sua vez fornece $\partial_{0} G(w, z)=0$ pelo Lema 1.1 .

Para a segunda afirmação temos pela eq. (2.30),

$$
\partial_{0}^{2} \Gamma=-\left.\Gamma \partial^{2} G \Gamma\right|_{\kappa_{p}=0}-\left.2 \Gamma \partial G \partial \Gamma\right|_{\kappa_{p}=0}
$$

Nós agora consideramos separadamente cada termo no lado direito da eq. (2.32). Para o 
primeiro termo temos

$$
\begin{aligned}
&\left.\Gamma \partial^{2} G \Gamma(u, v)\right|_{\kappa_{p}=0}=\left.\sum_{w, z} \Gamma(u, w) \partial^{2} G(w, z) \Gamma(z, v)\right|_{\kappa_{p}=0} \\
&=\left.\sum_{w, z \mid w^{0}+1 / 2 \leq p \leq z^{0}-1 / 2} \Gamma(u, w) \partial^{2} G(w, z) \Gamma(z, v)\right|_{\kappa_{p}=0} \\
&=2\left.\sum_{w, z \mid w^{0}+1 / 2 \leq p \leq z^{0}-1 / 2} \Gamma(u, w) \sum_{r \mid r^{0}+1 / 2=p} G(w, r) G\left(r+e^{0}, z\right) \Gamma(z, v)\right|_{\kappa_{p}=0} \\
&=\left.2 \sum_{w, z} \Gamma(u, w) \sum_{r \mid r^{0}+1 / 2=p} G(w, r) G\left(r+e^{0}, z\right) \Gamma(z, v)\right|_{\kappa_{p}=0} \\
&=2 \sum_{w \mid w^{0}=-1 / 2+p} \delta(u, w) \delta\left(w+e^{0}, v\right) .
\end{aligned}
$$

Para obtermos a segunda igualdade na equação acima usamos a primeira parte do Lema 2.1, i.e. $\left.\Gamma(u, v)\right|_{\kappa_{p}=0}=0$ se $u^{0} \neq v^{0}$, em particular, $\Gamma(u, w)=0$ se $w^{0}+1 / 2 \geq p$ e $\Gamma(z, v)=0$ se $z^{0} \geq p-1 / 2$. A terceira igualdade segue do Lema 1.2. A quarta segue usando novamente que $\Gamma(u, w)=0$ se $w^{0}+1 / 2 \geq p$ e $\Gamma(z, v)=0$ se $z^{0} \geq p-1 / 2$. A quinta igualdade segue da identidade $G \Gamma=1$.

Para o segundo termo temos

$$
\begin{aligned}
\left.\Gamma \partial G \partial \Gamma(u, v)\right|_{\kappa_{p}=0} & =\left.\sum_{w, z} \Gamma(u, w) \partial G(w, z) \partial \Gamma(z, v)\right|_{\kappa_{p}=0} \\
& =\left.\sum_{w, z \mid w^{0}+1 / 2 \leq p \leq z^{0}-1 / 2} \Gamma(u, w) \partial G(w, z) \partial \Gamma(z, v)\right|_{\kappa_{p}=0}=0 .
\end{aligned}
$$

seguindo os passos utilizados para mostrarmos a eq. (2.31).

Agora estamos prontos para demonstrar o Teorema 3.

Prova do Teorema 3.1: Como estamos interessados em aberturas de massa na primeira ordem não-nula, caculamos as contribuições para $G$ até $\mathcal{O}\left(\kappa^{4}\right)$. Consideramos a expansão do numerador e denominador de $G_{k l}(u, v)$ em potências do parâmetro de "hopping" $\kappa$, i.e., quando $u^{0} \leq v^{0}$

$$
G_{k l}(u, v)=\frac{1}{Z} \int \mu_{k}(u) \Pi_{l}(v) e^{-S} d \psi d \bar{\psi} d \mu(g) \equiv \frac{N}{D} .
$$

Considerando o denominador, $D \equiv Z$, para um ponto onde os elos chegam e saem em direções 
opostas a integração dos férmions fornece produtos de $\Gamma$ 's, que, pela propriedade (2.26) são zero. A primeira contribuição não-nula ocorre em $\kappa^{8}$, correspondendo a dois conjuntos de quatro elos em direções opostas formando um quadrado elementar ("loop").

Para o numerador, $N$, apresentamos explicitamente dois cálculos típicos: um para a contribuição de ordem $\kappa^{2}$ para $G_{11}(x)$, associada a $x=\epsilon e^{\rho}(|x|=1)$, e outra para o caso da contribuição angular para $G_{11}(x)$, quando $x=\epsilon e^{\rho}+\epsilon^{\prime} e^{\sigma}$. O caso $\epsilon=\epsilon^{\prime}$ e $\rho=\sigma$ é simples já que a propriedade (2.27) pode ser usada.

A contribuição de ordem $\kappa^{2}$ para o caso $|x=u-v|=1$, envolve dois elos, com orientações opostas conectando $u$ e $v$. Esquematicamente:

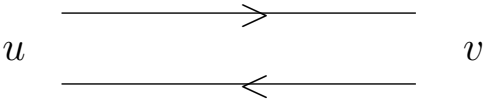

Usando a integral de calibre de dois campos na eq. (A.10) do Apêndice A, obtemos para $G_{11}(u, v)$ com \langle\rangle$^{(0)}$ denotando a média com o parâmetro de "hopping" $\kappa=0$ na ação (1.2), com a convenção da soma sobre índices repetidos,

$$
\frac{\kappa^{2}}{36}\left\langle\mu_{1} \bar{\psi}_{\alpha_{1}, a_{1}} \psi_{\beta_{2}, a_{1}}(u)\right\rangle^{(0)} \Gamma_{\alpha_{1} \beta_{1}}^{\epsilon \beta_{1}^{\rho}} \Gamma_{\alpha_{2} \beta_{2}}^{-\epsilon e^{\rho}}\left\langle\psi_{\beta_{1}, a_{2}} \bar{\psi}_{\alpha_{2}, a_{2}} \Pi_{1}(v)\right\rangle^{(0)}
$$

Usando as definições (2.4), (2.5) e aplicando novamente o Teorema de Wick para os fatores \langle\rangle$^{(0)}$, obtemos

$$
\frac{\kappa^{2}}{8}\left(\Gamma_{11}^{-\epsilon e^{\rho}} \Gamma_{33}^{\epsilon e^{\rho}}+\Gamma_{12}^{-\epsilon e^{\rho}} \Gamma_{43}^{\epsilon e^{\rho}}+\Gamma_{21}^{-\epsilon e^{\rho}} \Gamma_{34}^{\epsilon e^{\rho}}+\Gamma_{22}^{-\epsilon e^{\rho}} \Gamma_{44}^{\epsilon e^{\rho}}\right)
$$

Para $\epsilon=+1$ e $\rho=1$ temos as matrizes $\Gamma^{e^{1}}$ e $\Gamma^{-e^{1}}$, dadas pela eq. (1.3),

$$
\begin{aligned}
\Gamma^{1}=-1+\gamma^{1} & =\left(\begin{array}{cccc}
-1 & 0 & 0 & i \\
0 & -1 & i & 0 \\
0 & -i & -1 & 0 \\
-i & 0 & 0 & -1
\end{array}\right) \\
\Gamma^{-e^{1}}=-1-\gamma^{1} & =\left(\begin{array}{cccc}
-1 & 0 & 0 & -i \\
0 & -1 & -i & 0 \\
0 & i & -1 & 0 \\
i & 0 & 0 & -1
\end{array}\right)
\end{aligned}
$$


Pela estrutura das matrizes $\Gamma^{e^{1}}$ e $\Gamma^{-e^{1}}$, e desconsiderando o fator $\kappa^{2} / 8$, o segundo e terceiro termos do lado direito da eq. (2.33) são zero e a soma do primeiro e quarto é igual a 2. Desta forma, obtemos o resultado $\kappa^{2} / 4$ para a eq. (2.33). Para os outros casos, $G_{11}\left(x=v-u=e^{2}\right), G_{11}\left(x=v-u=e^{3}\right)$, partindo de $G_{11}\left(x=v-u=e^{1}\right)$, usamos rotações de $\pi / 2$ e $3 \pi / 2$ em relação a $e^{3}$ e $e^{2}$, respectivamente. As contribuições $-e^{j}(j=1,2,3)$ são obtidas a partir de $e^{j}$ pela simetria de paridade aplicada a $G_{11}\left(x=v-u=e^{j}\right)$. Finalmente, obtemos $G_{11}\left(x=\epsilon e^{i}\right)=G_{11}\left(x=e^{1}\right)=\kappa^{2} / 4$.

Para $\epsilon=+1$ e $\rho=0$ temos as matrizes $\Gamma^{e^{0}}$ e $\Gamma^{-e^{0}}$, pela eq. (1.3),

$$
\begin{aligned}
\Gamma^{e^{0}}=-1+\gamma^{0}= & \left(\begin{array}{cccc}
0 & 0 & 0 & 0 \\
0 & 0 & 0 & 0 \\
0 & 0 & -2 & 0 \\
0 & 0 & 0 & -2
\end{array}\right) \\
\Gamma^{-e^{0}}=-1-\gamma^{0} & =\left(\begin{array}{cccc}
-2 & 0 & 0 & 0 \\
0 & -2 & 0 & 0 \\
0 & 0 & 0 & 0 \\
0 & 0 & 0 & 0
\end{array}\right)
\end{aligned}
$$

e obtemos $\kappa^{2}$ para a eq. (2.33). Para o caso $G_{11}\left(x=v-u=-e^{0}\right)$, usando reversão no tempo do Teorema 1 em $G_{11}\left(x=v-u=e^{0}\right)$, obtemos $G_{11}\left(x=v-u=-e^{0}\right)=G_{11}\left(x=v-u=e^{0}\right)$. O resultado segue fazendo o cálculo para os demais elementos de matriz $G_{k l}(u, v), k$ e $l \neq 1$.

A contribuição de ordem $\kappa^{4}$ do ângulo $0 \rightarrow \epsilon e^{\rho} \rightarrow \epsilon e^{\rho}+\epsilon^{\prime} e^{\sigma} \equiv x$ para $G_{11}(u, v)=$ $G_{11}(x=v-u)$ é denotada por $A_{11}^{\epsilon e^{\rho}, x} \kappa^{4}$ e tem $g_{0, \epsilon e^{\rho}}\left(g_{0, \epsilon e^{\rho}}^{-1}\right)$ originando em $0\left(\epsilon e^{\rho}\right)$ na direção $e^{\rho}$ e $g_{\epsilon e^{\rho}, x}\left(g_{\epsilon e^{\rho}, x}^{-1}\right)$ chegando em $x\left(\epsilon e^{\rho}\right)$ na direção $e^{\sigma}$. Esquematicamente, esta contribuição corresponde a:

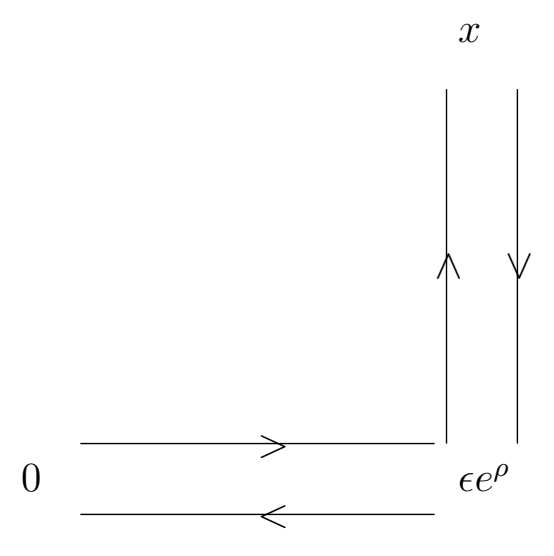

Depois de efetuarmos a integração nas variáveis de calibre para $g_{0, \epsilon e^{\rho}} g_{\epsilon e^{\rho}, 0}$ e $g_{\epsilon e^{\rho}, x} g_{x, \epsilon e^{\rho}}$ 
usando (A.10) e a integração dos férmions em $\epsilon e^{\rho}$ usando o Teorema de Wick, temos, com a convenção da soma sobre índices repetidos,

$$
\begin{aligned}
& A_{11}^{\epsilon e^{\rho}, x}=\frac{1}{144}\left\langle\mu_{1}(u) \bar{\psi}_{\alpha_{1}, a_{1}}(u) \psi_{\beta_{2}, a_{1}}(u)\right\rangle_{0}\left(\Gamma_{\alpha_{1} \beta_{1}}^{\epsilon \rho^{\rho}} \Gamma_{\alpha_{2} \beta_{2}}^{-\epsilon e^{\rho}} \Gamma_{\beta_{1} \beta_{3}}^{\epsilon^{\prime} e^{\sigma}} \Gamma_{\alpha_{4} \alpha_{2}}^{-\epsilon^{\prime} e^{\sigma}}\right. \\
& \left.-3 \Gamma_{\alpha_{1} \beta_{1}}^{\epsilon{ }^{\rho}} \Gamma_{\beta_{1} \beta_{2}}^{-\epsilon e^{\rho}} \Gamma_{\alpha_{3} \beta_{3}}^{\epsilon^{\prime} e^{\sigma}} \Gamma_{\alpha_{4} \alpha_{3}}^{-\epsilon^{\prime} e^{\sigma}}\right)\left\langle\psi_{\beta_{3}, a_{4}}(v) \bar{\psi}_{\alpha_{4}, a_{4}}(v) \Pi_{1}(v)\right\rangle_{0} .
\end{aligned}
$$

O segundo produto de $\Gamma^{\prime}$ 's acima é zero devido à propriedade (2.26). Usando as definições dos campos mesônicos dadas nas eqs. (2.4), (2.5), e aplicando novamente o Teorema de Wick para os fatores \langle\rangle$^{(0)}$, obtemos

$$
\begin{aligned}
& A_{11}^{\epsilon e^{\rho}, x}=\frac{1}{32}\left[\Lambda_{11}^{-\epsilon^{\prime} e^{\sigma},-\epsilon e^{\rho}} \Lambda_{33}^{\epsilon e^{\rho}, \epsilon^{\prime} e^{\sigma}}+\Lambda_{12}^{-\epsilon^{\prime} e^{\sigma},-\epsilon e^{\rho}} \Lambda_{43}^{\epsilon e^{\rho}, \epsilon^{\prime} e^{\sigma}}\right. \\
& \left.+\Lambda_{21}^{-\epsilon^{\prime} e^{\sigma},-\epsilon e^{\rho}} \Lambda_{34}^{\epsilon e^{\rho}, \epsilon^{\prime} e^{\sigma}}+\Lambda_{22}^{-\epsilon^{\prime} e^{\sigma},-\epsilon e^{\rho}} \Lambda_{44}^{\epsilon e^{\rho}, \epsilon^{\prime} e^{\sigma}}\right]
\end{aligned}
$$

onde usamos a notação $\Lambda_{u u^{\prime}}^{-\epsilon^{\prime} e^{\sigma},-\epsilon e^{\rho}} \equiv \Gamma_{u \alpha}^{-\epsilon^{\prime} e^{\sigma}} \Gamma_{\alpha u^{\prime}}^{-\epsilon e^{\rho}}$ e $\Lambda_{\ell \ell^{\prime}}^{\epsilon e^{\rho} \epsilon^{\prime} e^{\sigma}} \equiv \Gamma_{\ell \beta}^{\epsilon e^{\rho}} \Gamma_{\beta \ell^{\prime}}^{\epsilon^{\prime} e^{\sigma}}$, com a convenção da soma sobre índices repetidos. Similarmente, podemos calcular a contribuição de $A_{11}^{\epsilon^{\prime} e^{\sigma}, x}$. Esquematicamente esta contribuição corresponde a:

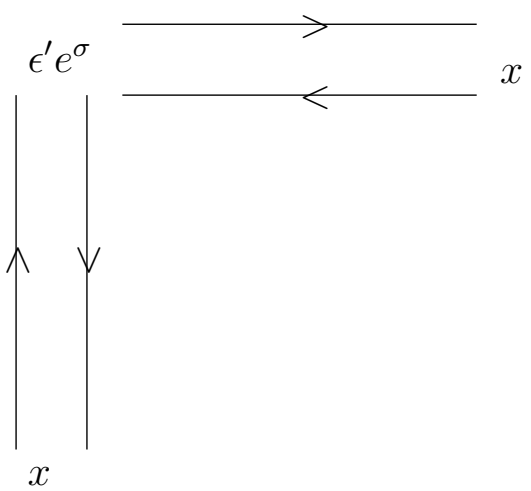

Finalmente, obtemos a expressão para $c_{11}(x)$,

$$
c_{11}(x)=A_{11}^{\epsilon e^{\rho}, x}+A_{11}^{\epsilon^{\prime} e^{\sigma}, x}
$$

$\operatorname{com} x=\epsilon e^{\rho}+\epsilon^{\prime} e^{\sigma}$, e similarmente para os outros $c_{k l}(x)^{\prime} s$. Quando $\rho=i$ e $\sigma=j(i, j=1,2,3)$ pelas propriedades de simetria do Teorema 1 (especificamente, rotações por $\pi / 2$ em relação a $e^{3}$ e paridade), podemos mostrar que $c_{k l}\left(x=\epsilon e^{i}+\epsilon^{\prime} e^{j}\right)$ é independente de $\epsilon, \epsilon^{\prime}$. Com isso, podemos escrever $c_{k l}(x)=c_{k l}\left(e^{i}+e^{j}\right) \equiv c_{k l}^{i j}$ e usando a estrutura explícita das matrizes $\Gamma$ 
obtemos a eq. (2.15).

Prova do Teorema 3.2: Usando a representação integral de Cauchy para cada $\kappa_{p}$ e levando em conta o número de derivadas que se anulam conforme o Lema 1 escrevemos, para $\kappa^{\prime}$ fixo suficientemente pequeno,

$$
G(u, v)=\prod_{u^{0}+1 / 2 \leq p \leq v^{0}-1 / 2}\left[\sum_{n_{p}=2} \int_{\left|w_{p}^{\prime}\right|=\kappa^{\prime}} d w_{p}^{\prime} \frac{w_{p}^{n_{p}}}{(2 \pi i) w_{p}^{\prime n_{p}+1}}\right] G\left(u, v ;\left\{w_{p}^{\prime}\right\}\right)
$$

e considerando $\left\{w_{p}\right\}=\kappa<\kappa^{\prime} / 2$ obtemos

$$
|G(u, v)|=c \prod_{u^{0}+1 / 2 \leq p \leq v^{0}-1 / 2}\left(\sum_{n_{p}=2}\left|\kappa / \kappa^{\prime}\right|^{n_{p}}\right) \leq c^{\prime}|\kappa|^{2\left|u^{0}-v^{0}\right|} .
$$

O resultado segue usando procedimento análogo para os parâmetros do desacoplamento de hiperplanos na direção espacial.

Então obtemos, pelo decaimento de $G$ no tempo e no espaço, temos

$$
\begin{aligned}
\left|\tilde{G}\left(p^{0}, \overrightarrow{0}\right)\right| & \leq \sum_{\left(x^{0}, \vec{x}\right) \in \mathbb{Z}^{d+1}}\left|G\left(x^{0}, \vec{x}\right)\right| e^{-\operatorname{Im} p^{0} x^{0}} \\
& \leq c^{\prime} \sum_{x^{0} \in \mathbb{Z}}\left|G\left(x^{0}, \overrightarrow{0}\right)\right| e^{-\operatorname{Im} p^{0} x^{0}} \\
& <c^{\prime \prime} \sum_{x^{0} \in \mathbb{Z}}\left|\kappa / \kappa^{\prime}\right|^{2\left|x^{0}\right|} e^{\operatorname{Im} p^{0}\left|x^{0}\right|}=c^{\prime \prime} \sum_{x^{0} \in \mathbb{Z}} e^{\left|x^{0}\right|\left[2 \ln \left(\kappa / \kappa^{\prime}\right)+\operatorname{Im} p^{0}\right]}
\end{aligned}
$$

o que implica na analiticidade de $\tilde{G}\left(p^{0}, \overrightarrow{0}\right)$ se $\left|\operatorname{Im} p^{0}\right|<-2 \ln \left(\kappa / \kappa^{\prime}\right)$. Quando $\kappa<\left(\kappa^{\prime}\right)^{2 / \epsilon}<<$ $\kappa^{\prime} / 2$ temos analiticidade na região $\left|\operatorname{Im} p^{0}\right|<-(2-\epsilon) \ln \kappa$.

Prova do Teorema 3.3: Para provarmos este item, usamos várias simetrias do Teorema 1. Nós aqui, apresentamos somente a prova da relação $G\left(e^{1}+e^{2}\right)=S G\left(e^{1}+e^{3}\right) S^{\dagger}$ onde $S$ está definido no enunciado do Teorema 3. Os outros resultados são obtidos de forma similar bastando para isto usarmos as simetrias: rotações de $\pi / 2$ e $\pi$ para os itens (a) e (b), respectivamente, e paridade para o item (c). Usando rotações de $\pi / 2$ em relação ao eixo $e_{1}$ de acordo com o Teorema 1, onde, para $\psi_{\alpha, a} \equiv \psi_{\alpha, a}\left(e^{1}+e^{2}\right), \psi_{\alpha, a}^{\prime} \equiv \psi_{\alpha, a}\left(r_{1}\left(e^{1}+e^{2}\right)\right)=\psi_{\alpha, a}\left(e^{1}+e^{3}\right)$, obtemos $\psi_{1, a} \mapsto(1-i)\left(\psi_{1, a}^{\prime}+i \psi_{2, a}^{\prime}\right) / 2, \psi_{2, a} \mapsto(1+i)\left(\psi_{1, a}^{\prime}-i \psi_{2, a}^{\prime}\right) / 2, \psi_{3, a} \mapsto(1-i)\left(\psi_{3, a}^{\prime}+i \psi_{4, a}^{\prime}\right) / 2$, $\psi_{4, a} \mapsto(1+i)\left(\psi_{3, a}^{\prime}-i \psi_{4, a}^{\prime}\right) / 2$ e para $\bar{\psi}$ nas expressões anteriores fazemos a mudança $\psi^{\prime} \mapsto \bar{\psi}^{\prime}$ e $i \mapsto-i$. Usando o fato que, a simetria de rotação de $\pi / 2$ em relação ao eixo $e^{1}$, é linear e preserva a ordem, obtemos, para os campos compostos $\mu$ e $\Pi, \mu_{k} \mapsto S_{j k} \mu_{k}^{\prime}$ e $\Pi_{k} \mapsto \Pi_{k}^{\prime} S_{j k}^{*}=$ $\Pi_{k}^{\prime}\left(S^{\dagger}\right)_{k j}$, de onde segue o resultado desejado.

Vamos agora provar o Teorema 4 que versa sobre o comportamento de $\Gamma$ a curtas e longas distâncias. 
Prova do Teorema 4.1: Nesta prova para deixar a notação mais compacta possível renomeamos as coordenadas temporais $u^{0} \mapsto u^{0}-1 / 2$ e $v^{0} \mapsto v^{0}-1 / 2$, neste caso, por exemplo, $G_{k k}(0,0)$ quer dizer $G_{k k}((1 / 2, \overrightarrow{0}),(1 / 2, \overrightarrow{0}))$. As correções ao valor assintótico da massa mesônica, $-2 \ln \kappa$, que precisamos para a determinação da abertura da massa, requerem valores precisos de $\Gamma_{k l}(x)$, para $|x|_{1}$ pequeno. Os resultados vão além daqueles obtidos pelo método de desacoplamento de hiperplanos, sendo obtidos através cancelamentos explícitos na série de Neumann para $\Gamma$. Os resultados abaixo são obtidos expandindo em potências de $\kappa$.

Nós consideramos o cálculo de $\Gamma$ para alguns valores de $x . \Gamma_{k l}$ é obtida pela série de Neumann e o primeiro item do Teorema 3. Relembrando que (veja eq. $(2.12)) G_{d, k l}(u, v)=$ $G_{k k}(u, u) \delta_{k l} \delta(u, v)$. Pelo Teorema 3.1, obtemos $G_{k k}(0)=1+\mathcal{O}\left(\kappa^{8}\right)$ e então $G_{k k}^{-1}(0)=1-\mathcal{O}\left(\kappa^{8}\right)$. Com $G_{n}$ dado na eq. (2.13), e usando $\Gamma=\sum_{i=0}^{\infty}(-1)^{i}\left[G_{d}^{-1} G_{n}\right]^{i} G_{d}^{-1}$, obtemos $x=0$ :

$$
\begin{aligned}
\Gamma_{k l}(0,0)= & G_{k k}^{-1}(0,0)-G_{k k}^{-1}(0,0) G_{n, k l}(0,0) G_{l l}^{-1}(0,0) \\
& +\sum^{\prime} G_{k k}^{-1}(0,0) G_{n, k m}(0, w) G_{m m}^{-1}(0,0) G_{n, m l}(w, 0) G_{l l}^{-1}(0,0)+\mathcal{O}\left(G_{n}^{3}\right)
\end{aligned}
$$

onde na eq. (2.34) $\sum^{\prime}$ significa soma em $w$ e $m$ com as restrições $w \neq 0$ ou $w=0 \mid k \neq m$ e $m \neq l$. Estas restrições são impostas de tal forma que possamos excluir os casos onde $G_{n, k m}(0, w)$ é identicamente nulo, i.e. $w=0$ e $k=m$. Nós agora consideramos o lado direito da eq. (2.34). Para o primeiro termo temos que $G_{k k}^{-1}(0,0)=1$ e para o segundo termo segue que $G_{n, k l}(0,0)=\mathcal{O}\left(\kappa^{8}\right)$. Para o terceiro termo temos contribuições da $\mathcal{O}\left(\kappa^{4}\right)$ com $w=\epsilon e^{\rho}$ $(\rho=0,1,2,3)$. Lembrando, do Teorema 3 , que na soma $\sum^{\prime}$ o fator $\kappa^{4}$ aparece duas vezes, correspondendo aos termos $w=\epsilon e^{0}(\epsilon= \pm 1)$, e $c_{2} \kappa^{4}$ aparece $2 d$ vezes correspondendo aos termos $w=\epsilon e^{i}(i=1,2$ para $d=2, i=1,2,3$ para $d=3$ e $\epsilon= \pm 1)$ e o resultado desejado segue.

$$
\begin{aligned}
x= & \epsilon e^{\rho}: \\
& \Gamma_{k l}\left(0, \epsilon e^{\rho}\right)=G_{d, k l}^{-1}\left(0, \epsilon e^{\rho}\right)-G_{k k}^{-1}(0,0) G_{n, k l}\left(0, \epsilon e^{\rho}\right) G_{l l}^{-1}\left(\epsilon e^{\rho}, \epsilon e^{\rho}\right) \\
& +\sum^{\prime \prime} G_{k k}^{-1}(0,0) G_{n, k m}(0, w) G_{m m}^{-1}(0,0) G_{n, m l}\left(w, \epsilon e^{\rho}\right) G_{l l}^{-1}\left(\epsilon e^{\rho}, \epsilon e^{\rho}\right)+\mathcal{O}\left(G_{n}^{3}\right) .
\end{aligned}
$$

onde na eq. (2.35) $\sum^{\prime \prime}$ significa soma em $w$ e $m$ com as restrições $w \neq 0$ e $w \neq e^{\rho}$ ou $w=e^{\rho} \mid k \neq m$ ou $w=0 \mid m \neq l$. Nós agora consideramos o lado direito da eq. (2.35). Para o primeiro termo termos que $G_{d, k l}^{-1}\left(0, \epsilon e^{\rho}\right)=0$. Para o segundo observamos pelo Teorema 3.1 que $G_{n, k l}\left(0, \epsilon e^{\rho}\right)=\kappa^{2} \delta_{\rho, 0}+c_{2} \kappa^{2} \delta_{\rho, i}(i=1,2$ para $d=2, i=1,2,3$ para $d=3)$. O terceiro termo é $\mathcal{O}\left(\kappa^{6}\right)$ se $w \neq 0, \mathcal{O}\left(\kappa^{10}\right)$ se $w=e^{\rho} \mid k \neq m$ e $\mathcal{O}\left(\kappa^{10}\right)$ se $w=0 \mid m \neq l$.

Agora, mostramos como os cancelamentos ocorrem no cálculo da série de Neumann para $\Gamma$, aprimorando as estimativas vindas do método de hiperplanos. Explicitamente consideramos o caso $x=\epsilon e^{0}+\epsilon^{\prime} e^{j}$; os outros casos onde temos uma, duas ou três unidades no tempo são 
tratados similarmente.

$$
\begin{aligned}
\Gamma_{k l}(u, v)= & -G_{k k}^{-1}(0) G_{n, k l}(u, v) G_{l l}^{-1}(0)+\sum_{w} G_{k k}^{-1}(0) G_{n, k m}(u, w) \\
& \times G_{m m}^{-1}(0) G_{n, m l}(w, v) G_{l l}^{-1}(0)+\mathcal{O}\left(G_{n}^{3}\right) .
\end{aligned}
$$

Para $k=l$, temos duas contribuições angulares de ordem $\kappa^{4}$ para $G_{n, k l}(u, v)$ no primeiro termo da eq. (2.36), e estes são cancelados pelo produto de contribuições de ordem $\kappa^{2}$ para $w-v=\epsilon e^{0}$ e $w-v=\epsilon e^{j}$ no segundo termo da eq. (2.36). Diagramaticamente podemos ver o cancelamento. Nas figuras abaixo a direção vertical é tomada como sendo a direção temporal e a horizontal como sendo espacial e $(\bullet-\bullet)$ corresponde ao termo $G_{n}$ :

Para o termo $G_{n}$ :
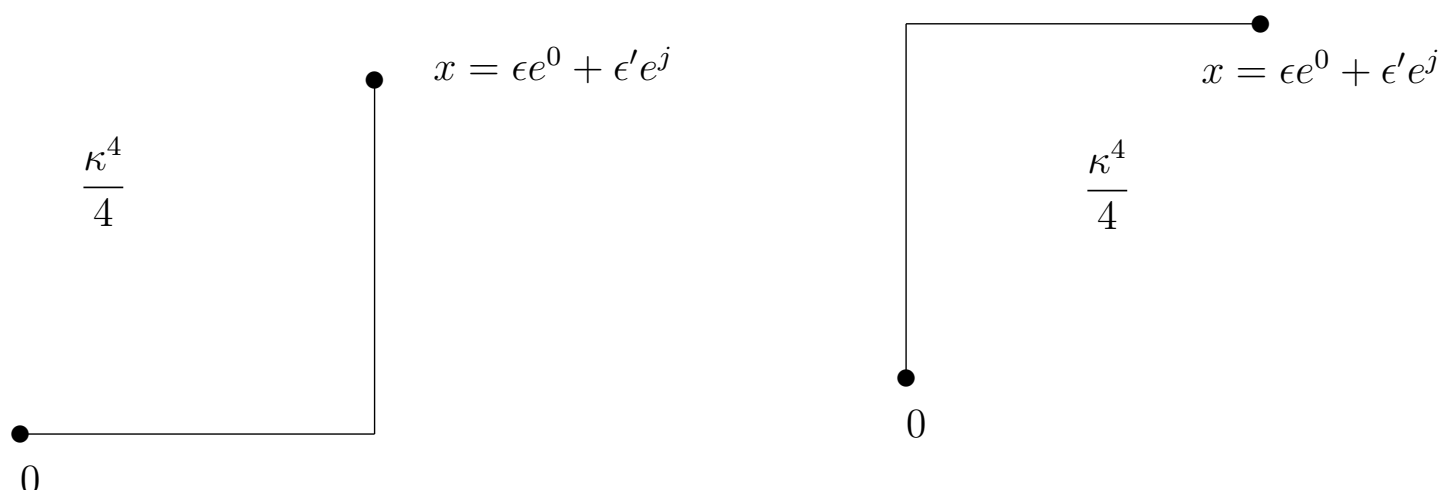

Para o termo $G_{n}^{2}$ :
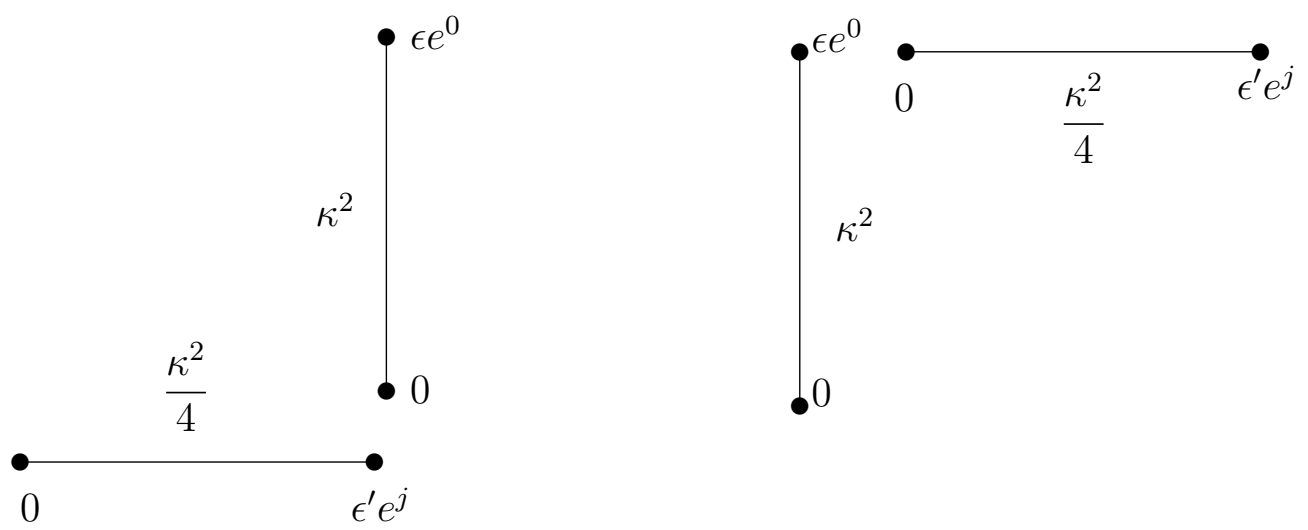
Prova do Teorema 4.2: A prova é similar à prova do segundo item do Teorema 3.

\subsection{Resultados Espectrais para um méson}

Para determinar as massas dos mésons e as curvas de dispersão, determinamos as soluções de det $\tilde{\Gamma}\left(p^{0}, \vec{p}\right)=0$, para $p^{0}$ no eixo imaginário. Para o espectro de massa mesônico, mostramos, usando simetrias, que $\tilde{\Gamma}_{k l}\left(p^{0}, \vec{p}=\overrightarrow{0}\right)$ é diagonal. Além disso, mostramos que $m_{j}+2 \ln \kappa(j=a, b)$ é real analítico em $\kappa$. Para $\vec{p} \neq \overrightarrow{0}$, como não achamos simetrias que simplificam a estrutura matricial de $\tilde{\Gamma}_{k l}\left(p^{0}, \vec{p}=\overrightarrow{0}\right)$, determinamos as curvas de dispersão $w(\vec{p})$, onde $\operatorname{det} \tilde{\Gamma}\left(p^{0}=i w(\vec{p}), \vec{p}\right)=0$, aplicando o Teorema de Rouché.

Apresentamos algumas propriedades de simetria no próximo Lema.

Lema 3 As seguintes propriedades de simetria valem para as matrizes $G$ e $\Gamma$.

1. $G_{k l}(x)=\left[G_{l k}(x)\right]^{*}$ e $\Gamma_{l k}(x)=\left[\Gamma_{k l}(x)\right]^{*}$;

2. For $\chi \in \mathbb{R}$, seja $p^{0}=i \chi$. Nós temos $\tilde{G}_{l k}(i \chi, \vec{p})=\left[\tilde{G}_{k l}(i \chi, \vec{p})\right]^{*}$ e $\tilde{\Gamma}_{l k}(i \chi, \vec{p})=\left[\tilde{\Gamma}_{k l}(i \chi, \vec{p})\right]^{*}$;

3. Quando $\vec{p}=\overrightarrow{0}, \tilde{G}_{l k}\left(p^{0}, \vec{p}=\overrightarrow{0}\right)=\operatorname{diag}[a+c, b, b, a-c], a, b, c \in \mathbb{C}$.

Prova do Lema 3: Para provar este Lema usamos várias simetrias do Teorema 1. Nós consideramos primeiramente o caso $x^{0} \neq 0$. Pela definição da função de dois pontos da eq. (2.6) obtemos, por exemplo, para $x^{0}=v^{0}-u^{0}<0, G_{l k}\left(x^{0}, \vec{x}\right)=\left\langle\mu_{l}(u) \Pi_{k}(v)\right\rangle=$ $\left\langle\Pi_{l}(\Theta u) \mu_{k}(\Theta v)\right\rangle^{*}=G_{l k}^{*}(\Theta x)=G_{l k}^{*}\left(-x^{0}, \vec{x}\right)$ usando reversão no tempo do Teorema 1. A seguir, usamos a representação espectral da eq. (2.10) que fornece $G_{l k}\left(-x^{0}, \vec{x}\right)=G_{k l}\left(x^{0}, \vec{x}\right)$. Daí segue que $G_{l k}\left(x^{0}, \vec{x}\right)=G_{l k}^{*}\left(-x^{0}, \vec{x}\right)=G_{k l}^{*}\left(x^{0}, \vec{x}\right)$. Para $x^{0}=0$, o resultado segue usando a definição $(2.6)$ que fornece $G_{l k}(0, \vec{x})=\left\langle\Pi_{l}(\Theta u) \mu_{k}(\Theta v)\right\rangle^{*}=\left\langle\mu_{k}\left(-u^{0}, \vec{v}\right) \Pi_{l}\left(-u^{0}, \vec{u}\right)\right\rangle^{*}$ usando reversão no tempo. A seguir usamos translação no tempo, espaço e paridade, para obtermos, $G_{l k}(0, \vec{x})=\left\langle\mu_{k}\left(u^{0}, \overrightarrow{0}\right) \Pi_{l}\left(u^{0}, \vec{u}-\vec{v}\right)\right\rangle^{*}=\left\langle\mu_{k}\left(u^{0}, \overrightarrow{0}\right) \Pi_{l}\left(u^{0}, \vec{v}-\vec{u}\right)\right\rangle^{*}=G_{k l}^{*}(0, \vec{x})$. Assim, o primeiro item é válido para todo $x$. Lembrando que

$$
\tilde{G}_{k l}(i \chi, \vec{p})=\sum_{x^{0}, \vec{x}} e^{\chi x^{0}} e^{-i \vec{p} \cdot \vec{x}} G_{k l}\left(x^{0}, \vec{x}\right),
$$

obtemos, pelo primeiro item deste Lema,

$$
\begin{aligned}
\tilde{G}_{k l}(i \chi, \vec{p}) & =\sum_{x^{0}, \vec{x}} e^{\chi x^{0}} e^{-i \vec{p} \cdot \vec{x}} G_{l k}^{*}\left(x^{0}, \vec{x}\right) \\
& =\left(\sum_{x^{0}, \vec{x}} e^{\chi x^{0}} e^{i \vec{p} \cdot \vec{x}} G_{l k}\left(x^{0}, \vec{x}\right)\right)^{*}=\tilde{G}_{l k}^{*}(i \chi,-\vec{p}),
\end{aligned}
$$


e, usando a simetria de paridade do Teorema $1, G_{k l}\left(x^{0}, \vec{x}\right)=G_{k l}\left(x^{0},-\vec{x}\right)$ que implica em $\tilde{G}_{k l}\left(p^{0}, \vec{p}\right)=\tilde{G}_{k l}\left(p^{0},-\vec{p}\right)$, a prova do segundo item segue. Para provarmos o terceiro item, reescrevemos a função de dois pontos mesônica da seguinte forma

$$
G_{k l}(u, v)=A_{k m} G_{m n}^{\prime}(u, v) A_{n l}^{-1}
$$

onde $G^{\prime}$ é a matriz definida por

$$
G_{m n}^{\prime}(u, v)=\left\langle M_{m}(u) \bar{M}_{n}(v)\right\rangle \chi_{u^{0} \leq v^{0}}+\left\langle\bar{M}_{m}(u) M_{n}(v)\right\rangle^{*} \chi_{u^{0}>v^{0}}
$$

onde fizemos a identificação $(1 / \sqrt{3}) \bar{\psi}_{\ell, a} \psi_{u, a} \rightarrow \bar{M}_{m}$ e $(1 / \sqrt{3}) \psi_{\ell, b} \bar{\psi}_{u, b} \rightarrow M_{n}$, de tal forma que $(\ell, u)=(3,1) \rightarrow m=1,(\ell, u)=(4,1) \rightarrow m=2,(\ell, u)=(3,2) \rightarrow m=3$ e $(\ell, u)=(4,2) \rightarrow$ $m=4$. $A$ é a matrix $4 \times 4$ dada por

$$
A \equiv\left(\begin{array}{rrrr}
1 / \sqrt{2} & 0 & 0 & 1 / \sqrt{2} \\
0 & 1 & 0 & 0 \\
0 & 0 & 1 & 0 \\
1 / \sqrt{2} & 0 & 0 & -1 / \sqrt{2}
\end{array}\right)=A^{-1}
$$

Considerando rotações de $\pi / 2$ em relação a $e^{3}$ e $d=3$ temos, $x=\left(x^{0}, x^{1}, x^{2}, x^{3}\right) \mapsto x^{\prime}=$ $\left(x^{0},-x^{2}, x^{1}, x^{3}\right)\left[x=\left(x^{0}, x^{1}, x^{2}\right) \mapsto x^{\prime}=\left(x^{0},-x^{2}, x^{1}\right)\right.$, para $\left.d=2\right]$ e $\tilde{\psi}_{1, a}(x) \mapsto \tilde{\psi}_{1, a}\left(x^{\prime}\right)$, $\psi_{2, a}(x) \mapsto-i \psi_{2, a}\left(x^{\prime}\right), \tilde{\psi}_{3, a}(x) \mapsto \tilde{\psi}_{3, a}\left(x^{\prime}\right), \psi_{4, a}(x) \mapsto-i \psi_{4, a}\left(x^{\prime}\right)$ e $\bar{\psi}_{2, a}(x) \mapsto i \bar{\psi}_{2, a}\left(x^{\prime}\right), \bar{\psi}_{4, a}(x) \mapsto$ $i \bar{\psi}_{4, a}\left(x^{\prime}\right)$. Para rotações de $\pi$ em relação a $e^{3}$ e $d=3$ temos, $x=\left(x^{0}, x^{1}, x^{2}, x^{3}\right) \mapsto x^{\prime}=$ $\left(x^{0},-x^{1},-x^{2}, x^{3}\right)\left[x=\left(x^{0}, x^{1}, x^{2}\right) \mapsto x^{\prime}=\left(x^{0},-x^{1},-x^{2}\right)\right.$, para $\left.d=2\right]$ e $\tilde{\psi}_{1, a}(x) \mapsto \tilde{\psi}_{1, a}\left(x^{\prime}\right)$, $\tilde{\psi}_{2, a}(x) \mapsto-\tilde{\psi}_{2, a}\left(x^{\prime}\right), \tilde{\psi}_{3, a}(x) \mapsto \tilde{\psi}_{3, a}\left(x^{\prime}\right), \tilde{\psi}_{4, a}(x) \mapsto-\tilde{\psi}_{4, a}\left(x^{\prime}\right)$. Usando estas simetrias e tomando a transformada de Fourier de $G^{\prime}(x=v-u)$ em $\left(p^{0}, \vec{p}=\overrightarrow{0}\right)$, obtemos a estrutura para $\tilde{G}^{\prime}$, onde $\times$ indica os elementos não nulos,

$$
\tilde{G}^{\prime}=\left(\begin{array}{cccc}
\times & 0 & 0 & \times \\
0 & \times & 0 & 0 \\
0 & 0 & \times & 0 \\
\times & 0 & 0 & \times
\end{array}\right) .
$$

A seguir, usando a simetria de reflexão em relação à coordenada $e^{1}$, ou seja, $\vec{x}=\left(x^{1}, \ldots, x^{d}\right) \mapsto$ $\vec{x}^{\prime}=\left(-x^{1}, \ldots, x^{d}\right)$, e $\tilde{\psi}_{1, a}(x) \mapsto-\tilde{\psi}_{2, a}\left(x^{\prime}\right), \tilde{\psi}_{2, a}(x) \mapsto-\tilde{\psi}_{1, a}\left(x^{\prime}\right), \tilde{\psi}_{3, a}(x) \mapsto \tilde{\psi}_{4, a}\left(x^{\prime}\right), \tilde{\psi}_{4, a}(x) \mapsto$ 
$\tilde{\psi}_{3, a}\left(x^{\prime}\right)$, obtemos relações entre os elementos não-nulos de $\tilde{G}^{\prime}$, i.e.

$$
\tilde{G}^{\prime}=\left(\begin{array}{cccc}
a & 0 & 0 & c \\
0 & b & 0 & 0 \\
0 & 0 & b & 0 \\
c & 0 & 0 & a
\end{array}\right)
$$

onde

$$
a \equiv \sum_{x=\left(x^{0}, \vec{x}\right)} e^{-i p^{0} x^{0}} G_{11}(x), b \equiv \sum_{x=\left(x^{0}, \vec{x}\right)} e^{-i p^{0} x^{0}} G_{33}(x) \text { e } c \equiv \sum_{x=\left(x^{0}, \vec{x}\right)} e^{-i p^{0} x^{0}} G_{44}(x) .
$$

Levando a eq. (2.38) na eq. (2.37) obtemos o resultado desejado.

Agora provamos o Teorema 5.

Prova do Teorema 5: Pelo Teorema 4, e tomando a transformada de Fourier de $\Gamma$ e fixando $\vec{p}=0$, temos

$$
\begin{gathered}
\tilde{\Gamma}_{k l}\left(p^{0}, \overrightarrow{0}\right)=\left[1-2 d c_{2} \kappa^{2}+2\left(1-d c_{2}-12 c_{2}^{2}+10 d c_{2}^{2}\right) \kappa^{4}\right] \delta_{\alpha \beta}-4\left(c_{k l}^{12}+\right. \\
\left.c_{k l}^{13}+c_{k l}^{23}\right) \kappa^{4}+\mathcal{O}\left(\kappa^{6}\right)-\delta_{k l}\left[\kappa^{2}+\mathcal{O}\left(\kappa^{8}\right)\right]\left(e^{i p^{0}}+e^{-i p^{0}}\right)+\ldots,
\end{gathered}
$$

Agora, introduzimos uma função auxiliar $H_{k}(w, \kappa)$, analítica em $w$ e $\kappa$, para $|\kappa|$ e $|w|$ suficientemente pequenos, tais que $H_{k}\left(w=1-\kappa^{2} e^{-i p^{0}}, \kappa\right)=\tilde{\Gamma}_{k k}\left(p^{0}, \vec{p}=\overrightarrow{0}\right) . H_{k}(w, \kappa)$ é definida por

$$
\begin{aligned}
H_{k}(w, \kappa)= & w-2 d c_{2} \kappa^{2}+2\left(1-d c_{2}-12 c_{2}^{2}+10 d c_{2}^{2}\right) \kappa^{4}-4\left(c_{k k}^{12}+c_{k k}^{13 ; 23}\right) \kappa^{4} \\
& -\frac{\kappa^{4}}{1-w}+\sum_{\vec{x}} \Gamma_{k k}^{\prime}(0, \vec{x})-\sum_{\vec{x}} \Gamma_{k k}^{\prime}(1, \vec{x})\left[\frac{\kappa^{2}}{1-w}+\frac{1-w}{\kappa^{2}}\right]+ \\
& \sum_{n \geq 1, \vec{x} \mid(n, \vec{x}) \neq(1, \overrightarrow{0})} \Gamma_{k k}(n, \vec{x})\left[\left(\frac{\kappa^{2}}{1-w}\right)^{n}+\left(\frac{1-w}{\kappa^{2}}\right)^{n}\right],
\end{aligned}
$$

onde $\Gamma_{k k}^{\prime}(0, \vec{x})$ (respectivamente, $\left.\Gamma_{k k}^{\prime}(1, \vec{x})\right)$ contém as contribuições de $\mathcal{O}\left(\kappa^{6}\right)$ (respectivamente, $\left.\mathcal{O}\left(\kappa^{8}\right)\right)$ ou de ordem superior e $c_{k k}^{13 ; 23}$ é a soma das contribuições angulares $0 \rightarrow e^{i} \rightarrow e^{13}$ $(i=1,3)$ e $0 \rightarrow e^{j} \rightarrow e^{23}(j=2,3)$, que é diagonal no índice de spin. $H_{k}$ satisfaz $H_{k}(0,0)=0$ e $\frac{\partial H_{k}}{\partial w}(0,0)=1$. Então, pelo Teorema da função implícita, existe uma função analítica $w(\kappa) \equiv$ $w_{k}(\kappa)$ tal que $H_{k}(w(\kappa), \kappa)=0$ e $w(0)=0$. Deste modo, para $\kappa$ real e positivo, a massa é dada por

$$
m_{k}=-\ln \kappa^{2}+\ln (1-w) .
$$

Nós observamos que $\ln (1-w)$ admite a expansão em série de Taylor $\sum_{n=1}^{\infty} c_{n} \kappa^{n}$ com $c_{n}=d_{0}^{n} \ln (1-w) / n$ !, onde usamos a notação compacta $d_{0}^{n} \equiv d^{n} /\left.d \kappa^{n}\right|_{\kappa=0}$. Os primeiros três 
coeficientes, $c_{0}, c_{1}$ e $c_{2}$, da expansão de $\ln (1-w)$ são dados explicitamente por

$$
\begin{gathered}
c_{0}=\frac{1}{0 !} d_{0}^{0} \ln [1-w(\kappa)]=\ln [1-w(0)]=\ln 1=0, \\
c_{1}=\frac{1}{1 !} \frac{1}{1-w(0)} d_{0}^{1} w(\kappa)=d_{0}^{1} w(\kappa), \\
c_{2}=-\frac{1}{2 !}\left\{\frac{1}{[1-w(0)]^{2}}\left[d_{0}^{1} w(\kappa)\right]^{2}+\frac{1}{1-w(0)} d_{0}^{2} w(\kappa)\right\}=-\frac{1}{2 !}\left\{\left[d_{0}^{1} w(\kappa)\right]^{2}+d_{0}^{2} w(\kappa)\right\},
\end{gathered}
$$

e assim por diante.

Para determinarmos os $d^{r} w$ analisamos as derivadas da função implícita obtidas diferenciando a equação $H_{k}(w(\kappa), \kappa)=0$ em relação a $\kappa$, ou seja

$$
0=d H_{\alpha}(w(\kappa), \kappa)=\left(\partial_{w} H_{\alpha}\right) d_{\kappa} w+\partial_{\kappa} H
$$

de onde obtemos

$$
d w=-\left(\partial_{w} H\right)^{-1} \partial_{k} H \Rightarrow d_{0}^{1} w=0,
$$

pela eq. (2.40).

De forma similar, obtemos para $d_{0}^{r} w=0, r=3,5 \mathrm{e}$

$$
\begin{aligned}
& d_{0}^{2} w=-\partial_{\kappa}^{2} H(0,0)=4 c_{2} d \\
& d_{0}^{4} w=-\partial_{\kappa}^{4} H(0,0)=-4 !\left[1-2 d c_{2}-24 c_{2}^{2}+20 d c_{2}^{2}-4\left(c_{k k}^{12}+c_{k k}^{13 ; 23}\right)\right] .
\end{aligned}
$$

Então, para $d=3$,

$$
\begin{aligned}
m_{k}= & -2 \ln \kappa-2 d c_{2} \kappa^{2}+ \\
& {\left[4\left(c_{k k}^{12}+c_{k k}^{13 ; 23}\right)-\left(1-2 d c_{2}-24 c_{2}^{2}+20 d c_{2}^{2}\right)-2 d^{2} c_{2}^{2}\right] \kappa^{4}+\mathcal{O}\left(\kappa^{6}\right), }
\end{aligned}
$$

e similarmente, para $d=2, \operatorname{com} c_{k k}^{13 ; 23}=0$.

Então, até (e incluindo) a ordem $\mathcal{O}\left(\kappa^{4}\right)$, temos, para $d=3, m_{a} \equiv m_{1}$ e $m_{b} \equiv m_{k}$, $m_{b}-m_{a}=2 \kappa^{4}$ e $k=2,3,4$; para $d=2, m_{a} \equiv m_{k}$ and $m_{b} \equiv m_{l}, m_{b}-m_{a}=\kappa^{4}, k=2,3 \mathrm{e}$ $l=1,4$.

Vamos agora considerar as curvas de dispersão. Elas satisfazem a equação $\operatorname{det} \tilde{\Gamma}\left(p^{0}=\right.$ $i w(\vec{p}), \vec{p})=0$. Para determiná-las, com $c_{2}(\vec{p}) \equiv c_{2} \sum_{j=1}^{d} 2 \cos p^{j}$, escrevemos a transformada de Fourier de $\Gamma_{k l}(x)$ como

$$
\begin{aligned}
\tilde{\Gamma}_{k l}\left(p^{0}, \vec{p}\right)= & {\left[1-c_{2}(\vec{p}) \kappa^{2}-\kappa^{2}\left(e^{-i p^{0}}+e^{i p^{0}}\right)\right] \delta_{k l} } \\
& +\sum_{n, \vec{x}}^{\prime} \Gamma_{k l}(n, \vec{x}) e^{-i \vec{p} \cdot \vec{x}}\left(e^{-i p^{0} n}+e^{i p^{0} n}\right),
\end{aligned}
$$


onde $\sum_{n, \vec{x}}$ 'significa que todos os termos de ordem $\kappa^{4}$ ou superior em $\Gamma_{k l}(x)$ são incluídos. Introduzindo a função matricial auxiliar $H_{k l}(w, \kappa) \equiv H_{k l}(w, \kappa, \vec{p})$ tal que $H_{k l}\left(w=1-c_{2}(\vec{p}) \kappa^{2}-\right.$ $\left.\kappa^{2} e^{-i p^{0}}, \kappa\right)=\tilde{\Gamma}_{k l}\left(p^{0}, \vec{p}\right), H_{k l}(w, \kappa)$ é definida por

$$
\begin{aligned}
H_{k l}(w, \kappa)= & w \delta_{k l}+\sum_{n, \vec{x}}^{\prime \prime} \Gamma_{k l}(n, \vec{x}) e^{-i \vec{p} \cdot \vec{x}} \times \\
& {\left[\left(\frac{1-w-c_{2}(\vec{p}) \kappa^{2}}{\kappa^{2}}\right)^{n}+\left(\frac{\kappa^{2}}{1-w-c_{2}(\vec{p}) \kappa^{2}}\right)^{n}\right], }
\end{aligned}
$$

onde $\sum_{n, \vec{x}}$ " significa os termos de ordem $\mathcal{O}\left(\kappa^{4}\right)$ ou superior são incluídos. $H(w, \kappa)$ é analítica em $\kappa$ e $w$ em $(w, \kappa)=(0,0)$.

Tomando

$$
\begin{aligned}
f(w) \equiv \operatorname{det} H(w, \kappa) & =\operatorname{det} w I_{4}+\left[\operatorname{det} H(w, \kappa)-\operatorname{det} w I_{4}\right] \\
& \equiv g(w)+h(w)
\end{aligned}
$$

podemos aplicar o Teorema de Rouché a $f(w)$ no círculo $|w|=c|\kappa|^{4}, c \ggg 1,|g(w)|=c^{4}|\kappa|^{16}$ e $|h(w)| \leq c^{\prime}|\kappa|^{16}<c^{4}|\kappa|^{16}=|g(w)|$. Dele segue que $f(w)$ tem quatro zeros dentro de $|w|=c|\kappa|^{4}$, já que $g(w)=w^{4}$ tem um zero de quarta ordem. Note que a cota superior para $|h(w)|$ vem através de uma cota superior nos restantes vinte e três termos na diferença entre os dois determinantes das matrizes $4 \times 4 H(w, \kappa)$ e $w I_{4}$. Agora, para $p^{0}=i w(\vec{p})$, e $\kappa$ real positivo, cada um dos quatro zeros satisfazendo $\operatorname{det} \tilde{\Gamma}\left(p^{0}=i w(\vec{p}), \vec{p}\right)=0$ tem a forma

$$
w(\vec{p})=-2 \ln \kappa-2 d c_{2} \kappa^{2}+2 c_{2} \kappa^{2} \sum_{j=1}^{d}\left(1-\cos p^{j}\right)+\mathcal{O}\left(\kappa^{4}\right)
$$

Agora, estendemos os resultados espectrais para uma partícula mesônica de $\mathcal{H}_{m}$ para todo $\mathcal{H}_{e}$, usando o método de subtração euclideana estabelecido em [31]. Começamos considerando a seguinte função de correlação generalizada

$$
\mathcal{F}(u, v)=\mathcal{G}_{L, L}(u, v)-\sum_{w, w^{\prime} \in \mathbb{Z}_{0}^{d+1}} \mathcal{G}_{L, \Phi}(u, w) \Gamma\left(w, w^{\prime}\right) \mathcal{G}_{\Phi, L}\left(w^{\prime}, v\right)
$$

onde $\Phi(u)=\left(\Pi_{1}(u), \ldots, \Pi_{4}(u)\right)$ tem quatro componentes. Relembrando, $\Gamma\left(w, w^{\prime}\right)$ é dada pela inversa convolutiva da função de dois pontos $G$ e $\mathcal{G}_{L, M}$ corresponde à função definida na Proposição 1.

A razão da escolha da forma funcional do lado direito da eq. (2.42) reside nos seguintes fatos:

- As possíveis singularidades em $\left|\operatorname{Im} p^{0}\right|<-(4-\epsilon) \ln \kappa$ da transformada de Fourier de $\mathcal{F}(x=v-u)=\mathcal{F}(u, v)$ são canceladas. 
- As possíveis singularidades na transformada de Fourier de $\mathcal{G}_{L, L}(x=v-u)=\mathcal{G}_{L, L}(u, v)$ vem de $\omega(\vec{p})$.

Conforme veremos no Lema 4 (a seguir), temos $\partial_{p}^{r} \mathcal{F}(u, v)=0(r=0, \ldots, 4)$ e, assim, $|F(u, v)| \leq e^{-(4-\epsilon) \ln \kappa\left|v^{0}-u^{0}\right|}$, o quê implica na analiticidade de $\tilde{F}(p)$, a transformada de Fourier de $F(0, x)$, em $\left|\operatorname{Im} p^{0}\right|<-(4-\epsilon) \ln \kappa$, seguindo os passos do Teorema 3. Para $\tilde{F}(p)$ obtemos $\tilde{F}(p)=\tilde{\mathcal{G}}_{L, L}(p)+\tilde{\mathcal{G}}_{L, \Phi}(p) \tilde{\Gamma}(p) \tilde{\mathcal{G}}_{\Phi, L}(p)$. $\tilde{\mathcal{G}}_{L, \Phi}(p)$ e $\tilde{\mathcal{G}}_{\Phi, L}(p)$ têm representações espectrais similares a de $\tilde{G}(p)$ com singularidades dadas por $\omega(\vec{p})$ em $\left|\operatorname{Im} p^{0}\right|<-(4-\epsilon) \ln \kappa$ (Teorema 5$)$. $\tilde{\Gamma}(p)$ é analítica nesta região pelo Teorema 4. Resulta, então, que as singularidades de $\tilde{\mathcal{G}}_{L, L}(p)$ são dadas por $\omega(\vec{p})$.

Desta forma, o Lema abaixo garante que nossos resultados espectrais são válidos não só em $\mathcal{H}_{m}$, mas em todo espaço $\mathcal{H}_{e}$.

Lema 4 Para $u^{0}<v^{0}, p \in \mathbb{Z}, u^{0}+1 / 2 \leq p \leq v^{0}-1 / 2$ (ou, se $u^{0}>v^{0}, v^{0}+1 / 2 \leq p \leq$ $\left.u^{0}-1 / 2\right)$, e denotando novamente por $\partial_{0}$ a derivada em relação a $\kappa_{p}$ em $\kappa_{p}=0$, temos $\partial_{0}^{r} \mathcal{F}(u, v)=0$, para $r=0,1,2,3$.

Prova do Lema 4: Para $u^{0}<v^{0}$ (o caso $u^{0}>v^{0}$ é similar), dos Lemas 1 e 2, as expansões em potências de $\kappa_{p}$ das funções que aparecem na definição de $\mathcal{F}(u, v)$ têm a forma

$$
\begin{aligned}
\mathcal{G}_{L, L}(u, v)= & a_{2}(u, v) \kappa_{p}^{2}+\mathcal{O}\left(\kappa_{p}^{4}\right) \\
\mathcal{G}_{L, \Phi}(u, w)= & b_{0}(u, w) \chi_{w^{0} \leq-1 / 2+p}+b_{2}(u, w) \kappa_{p}^{2}+\mathcal{O}\left(\kappa_{p}^{4}\right) \\
\Gamma\left(w, w^{\prime}\right)= & c_{0}\left(w, w^{\prime}\right)\left(\chi_{w^{0} \leq-1 / 2+p} \chi_{w^{\prime 0} \leq-1 / 2+p}+\chi_{w^{0}>-1 / 2+p}\right. \\
& \left.\times \chi_{w^{\prime 0}>-1 / 2+p}\right)+c_{2}\left(w, w^{\prime}\right) \kappa_{p}^{2}+\mathcal{O}\left(\kappa_{p}^{4}\right) \\
\mathcal{G}_{\Phi, L}\left(w^{\prime}, v\right)= & d_{0}\left(w^{\prime}, v\right) \chi_{w^{\prime 0}>-1 / 2+p}+d_{2}\left(w^{\prime}, v\right) \kappa_{p}^{2}+\mathcal{O}\left(\kappa_{p}^{4}\right),
\end{aligned}
$$

onde $a_{2}(u, v) \equiv \partial_{0}^{2} \mathcal{G}_{L, L}(u, v)$, e assim por diante. Substituindo estas expressões na eq. (2.42), é fácil verificar que $\partial_{0}^{r} \mathcal{F}(u, v)=0(r=0,1,3)$. Para $r=2$, temos

$$
\begin{aligned}
\partial_{0}^{2} \mathcal{F}(u, v)= & \partial_{0}^{2} \mathcal{G}_{L, L}(u, v)-\sum_{w, w^{\prime} \in \mathbb{Z}_{0}^{d+1}} \partial_{0}^{2} \mathcal{G}_{L, \Phi}(u, w) \Gamma\left(w, w^{\prime}\right) \mathcal{G}_{\Phi, L}\left(w^{\prime}, v\right) \\
& -\sum_{w, w^{\prime} \in \mathbb{Z}_{0}^{d+1}} \mathcal{G}_{L, \Phi}(u, w) \partial_{0}^{2} \Gamma\left(w, w^{\prime}\right) \mathcal{G}_{\Phi, L}\left(w^{\prime}, v\right) \\
& -\sum_{w, w^{\prime} \in \mathbb{Z}_{0}^{d+1}} \mathcal{G}_{L, \Phi}(u, w) \Gamma\left(w, w^{\prime}\right) \partial_{0}^{2} \mathcal{G}_{\Phi, L}\left(w^{\prime}, v\right)
\end{aligned}
$$

Usando os Lemas 1 e 2 com, $u^{0}+1 / 2 \leq p<v^{0}-1 / 2$, obtemos

$$
\partial_{0}^{2} \Gamma\left(w, w^{\prime}\right)=-2 \sum_{z \mid z^{0}=-1 / 2+p} \delta(w, z) \delta\left(w^{\prime}, z+e^{0}\right)
$$




$$
\partial_{0}^{2} \mathcal{G}_{L, M}(u, v)=2 \sum_{z \mid z^{0}=-1 / 2+p}\left\langle L(u) \Pi_{\alpha}(z)\right\rangle\left\langle\mu_{\alpha}\left(z+e^{0}\right) M(v)\right\rangle
$$

onde $L, M \in \mathcal{H}_{e}$. Em seguida, procedendo como na prova do Lema 2 e usando a eq. (2.44) para $\partial_{0}^{2} \mathcal{G}_{L, L}, \partial_{0}^{2} \mathcal{G}_{\Phi, L}$ e $\partial_{0}^{2} \mathcal{G}_{L, \Phi}$, obtemos para o lado direito da eq. (2.43) que o primeiro termo se cancela com o segundo e o terceiro com o último. O resultado desejado segue desta propriedade.

Isto encerra a prova do Teorema 5.

Estabelecidos os resultados espectrais para uma partícula mesônica e usando os resultados para uma partícula bariônica enunciados no Teorema 6 e provados em [12], passamos à consideração da existência de estados ligados de dois mésons e de um méson e um bárion nos próximos capítulos. 


\section{Capítulo 3}

\section{Ausência de estados ligados de méson-méson e méson-bárion em $(2+1)$ dimensões, um sabor e matrizes de Pauli $2 \times 2$}

Neste capítulo, em $2+1$ dimensões, usando matrizes de spin $2 \times 2$, consideramos a existência de estados ligados do tipo méson-méson e méson-bárion abaixo do limiar de mésonméson $(\sim-4 \ln \kappa)$ e méson-bárion $(\sim-5 \ln \kappa)$, respectivamente. Para tal, necessitamos de resultados para o espectro de um méson e um bárion tal qual enunciados e demonstrados no Capítulo 2, adaptados para o caso de matrizes de spin $2 \times 2$. Essencialmente, a menos das análises das aberturas de massa dadas em [12, 19], os resultados para as curvas de dispersão e massas continuam os mesmos ao menos até ordem $\kappa^{2}$, para mésons, e ao menos até ordem $\kappa^{3}$, para bárions (para este caso, ver [11]).

Dividimos este capítulo em quatro seções. Na primeira seção, usamos a representação espectral para a função de dois pontos mesônica e bariônica obtidas a partir da Proposição 1 para a determinarmos a função de dois pontos para mésons e bárions. Em seguida, introduzimos a função de quatro pontos para o méson-méson e o méson-bárion. Nas Seções 3 e 4, respectivamente, introduzimos e resolvemos a equação de B-S na aproximação escada para o méson-méson. Mostramos a correspondência entre a equação de B-S, na aproximação escada, em coordenadas relativas, para o sistema com momento espacial zero, e a equação de Schrödinger na forma resolvente. Para o méson-méson, a aproximação escada corresponde a um potencial não-local no espaço e com alcance um. Finalizamos este capítulo com a Seção 4, apresentando resultados similares para a existência de estados ligados do tipo méson-bárion. Neste caso, restringindo nossa análise ao setor de paridade positiva obtemos um potencial local e de alcance um no espaço. Resolvendo a equação de B-S mostramos também que não 
existem estados ligados do tipo méson-bárion.

Neste capítulo para deixar a notação a mais clara possível, usaremos o superscrito $m$ (b) para nos referir a mésons (bárions).

Os resultados deste capítulo foram publicados em dois artigos [14, 20].

\subsection{A função parcialmente truncada de quatro pontos para mésons e méson-bárion}

Para analisarmos a existência do estado ligado de um méson e um bárion, nós apresentamos resultados espectrais para partículas mesônicas e bariônicas.

Em $(2+1)$ dimensões, um sabor e usando matrizes de spin $2 \times 2$ os campos mesônicos são dados por, com $u \in \mathbb{Z}_{o}^{d+1}$,

$$
\Pi(u)=(1 / \sqrt{3}) \bar{\psi}_{-, a}(u) \psi_{+, a}(u) ; \mu(u)=(1 / \sqrt{3}) \psi_{-, a}(u) \bar{\psi}_{+, a}(u)
$$

Na definição (3.1) os índices de spin para o campo $\Pi(\mu)$ é $+(-)$ para o quark e $-(+)$ para o anti-quark.

Os campos bariônicos são dados por, com $u \in \mathbb{Z}_{o}^{d+1}$,

$$
\phi_{-}(u)=\frac{1}{6} \epsilon_{a b c} \psi_{-, a}(u) \psi_{-, b}(u) \psi_{-, c}(u)=\psi_{-, 1}(u) \psi_{-, 2}(u) \psi_{-, 3}(u)
$$

$\mathrm{e}$

$$
\bar{\phi}_{-}(u)=\frac{1}{6} \epsilon_{a b c} \bar{\psi}_{-, a}(u) \bar{\psi}_{-, b}(u) \bar{\psi}_{-, c}(u)=\bar{\psi}_{-, 1}(u) \bar{\psi}_{-, 2}(u) \bar{\psi}_{-, 3}(u) .
$$

Pela simetria de conjugação de carga do Teorema 2 temos $C \bar{\phi}_{-}\left(\phi_{-}\right)=i \phi_{+}\left(-i \bar{\phi}_{+}\right)$e $C \Pi(\mu)=$ $-\Pi(-\mu)$ onde $\phi_{+}\left(\bar{\phi}_{+}\right)$é obtido a partir de $\phi_{-}\left(\bar{\phi}_{-}\right)$substituindo o índice de spin - pelo índice + nos campos fermiônicos simples. Nós referimos a $\bar{\phi}_{+}(u)$ como a anti-partícula bariônica e os mésons são suas próprias anti-partículas, lembrando que $C \mu(\Pi)=-\mu(-\Pi)$. De agora em diante, suprimimos o índice de spin - de $\bar{\phi}_{-}$. Considerando a fórmula de F-K da Proposição 1 temos

$$
\left(\Phi^{j}\left(1 / 2, \vec{x}_{1}\right), \check{T}_{0}^{\left|x^{0}\right|-1} \Phi^{j}\left(1 / 2, \vec{x}_{2}\right)\right)_{\mathcal{H}}=\int_{-1}^{1} \int_{\mathbf{T}^{d}}\left(\lambda^{0}\right)^{\left|x^{0}\right|-1} e^{i \vec{\lambda}_{.}\left(\vec{x}_{2}-\vec{x}_{1}\right)} d\left(\Phi^{j}, \mathcal{E}\left(\lambda^{0}, \vec{\lambda}\right) \Phi^{j}\right)_{\mathcal{H}}
$$

$x^{0} \neq 0$, e $\Phi^{m}=\Pi\left(\Phi^{b}=\bar{\phi}\right)$. Nós notamos, pela representação espectral da eq. (3.4), que $\Pi$, $\bar{\phi}$ geram partículas e $\mu, \phi$ são campos auxiliares que entram na definição das funções de dois pontos. 
Agora, nós definimos as funções de dois pontos para mésons

$$
\begin{aligned}
G^{m}\left(u^{0}, \vec{x}_{1} ; v^{0}, \vec{x}_{2}\right) & =\chi_{u^{0} \leq v^{0}}\left\langle\mu\left(u^{0}, \vec{x}_{1}\right) ; \Pi\left(v^{0}, \vec{x}_{2}\right)\right\rangle_{T}+\chi_{u^{0}>v^{0}}\left\langle\Pi\left(u^{0}, \vec{x}_{1}\right) ; \mu\left(v^{0}, \vec{x}_{2}\right)\right\rangle_{T}^{*} \\
& =\chi_{u^{0} \leq v^{0}}\left\langle\mu\left(u^{0}, \vec{x}_{1}\right) \Pi\left(v^{0}, \vec{x}_{2}\right)\right\rangle+\chi_{u^{0}>v^{0}}\left\langle\Pi\left(u^{0}, \vec{x}_{1}\right) \mu\left(v^{0}, \vec{x}_{2}\right)\right\rangle^{*},
\end{aligned}
$$

e para bárions

$$
G^{b}\left(u^{0}, \vec{x}_{1} ; v^{0}, \vec{x}_{2}\right)=-\chi_{u^{0} \leq v^{0}}\left\langle\phi\left(u^{0}, \vec{x}_{1}\right) \bar{\phi}\left(v^{0}, \vec{x}_{2}\right)\right\rangle+\chi_{u^{0}>v^{0}}\left\langle\bar{\phi}\left(u^{0}, \vec{x}_{1}\right) \phi\left(v^{0}, \vec{x}_{2}\right)\right\rangle^{*}
$$

onde $x^{0}=v^{0}-u^{0} \in \mathbb{Z}$. A função de dois pontos para o anti-bárion é obtida a partir de $G^{b}$ pela aplicação da conjugação de carga do Teorema 2 .

Procedendo como na demonstração do Teorema 5, podemos mostrar que o espectro de energia-momento para as partículas mesônicas em $(2+1)$ dimensões, um sabor e matrizes de spin $2 \times 2$, têm curvas de dispersão isoladas dadas por

$$
\begin{aligned}
w^{m}(\vec{p}) & =-2 \ln \kappa+r^{m}(\kappa, \vec{p})=-2 \ln \kappa+\ln \left[1-\frac{\kappa^{2}}{2}\left(\cos p^{1}+\cos p^{2}\right)\right]+\mathcal{O}\left(\kappa^{4}\right) \\
& =-2 \ln \kappa-\kappa^{2}+c^{m} \kappa^{2}[-\tilde{\Delta}(\vec{p})]+\mathcal{O}\left(\kappa^{4}\right)
\end{aligned}
$$

com, $c_{m}=1 / 4, r^{m}(\kappa, \vec{p})$ real e analítica em $\kappa$ e em cada componente $p^{j}(j=1,2)$. $[-\tilde{\Delta}(\vec{p})]=$ $2 \sum_{i=1,2}\left(1-\cos p^{i}\right)$ é a transformada de Fourier do Laplaciano em $\ell_{2}\left(\mathbb{Z}^{2}\right)$. $w^{m}(\vec{p}) \approx m^{m}+$ $\frac{\kappa^{2}}{4}|\vec{p}|^{2},|\vec{p}| \ll 1$, onde $m^{m} \equiv m^{m}(\kappa)=w(\overrightarrow{0})$ é a massa do méson.

Para as partículas bariônicas, os seguintes resultados valem

$$
\begin{aligned}
w^{b}(\vec{p}) & =-3 \ln \kappa+r^{b}(\kappa, \vec{p})=-3 \ln \kappa+\ln \left[1-\frac{\kappa^{3}}{4}\left(\cos p^{1}+\cos p^{2}\right)\right]+\mathcal{O}\left(\kappa^{4}\right) \\
& =-3 \ln \kappa-\kappa^{3}+c^{b} \kappa^{3}[-\tilde{\Delta}(\vec{p})]+\mathcal{O}\left(\kappa^{4}\right)
\end{aligned}
$$

com, $c^{b}=1 / 8, r^{b}(\kappa, \vec{p})$ real e analítica em $\kappa$ e em cada componente $p^{j}(j=1,2)$. $w^{b}(\vec{p}) \approx$ $m^{b}+\frac{\kappa^{3}}{8}|\vec{p}|^{2},|\vec{p}| \ll 1$, onde $m^{b} \equiv m^{b}(\kappa)=w^{b}(\overrightarrow{0})$ é a massa do bárion.

Nós agora passamos à consideração da medida espectral $d \sigma_{\vec{\lambda}}^{j}\left(\lambda^{0}\right) \equiv d\left(\Phi^{j}, \mathcal{E}\left(\lambda^{0}, \vec{\lambda}\right) \Phi^{j}\right)_{\mathcal{H}}$ da eq. (3.4). Uma versão do Teorema enunciado abaixo, foi obtida em [32] para o modelo de Ising no regime de baixas temperaturas. Adaptando ao nosso modelo, temos o Teorema que versa sobre a decomposicão da medida espectral $d \sigma_{\vec{\lambda}}^{j}\left(\lambda^{0}\right)$.

Teorema $7 d \sigma_{\vec{\lambda}}^{j}\left(\lambda^{0}\right)=Z^{j}(\vec{\lambda}) \delta\left(\lambda^{0}-e^{-w^{j}(\vec{\lambda})}\right) d \lambda^{0}+d \hat{\sigma}_{\vec{\lambda}}^{j}\left(\lambda^{0}\right)$. d $\hat{\sigma}_{\vec{\lambda}}^{m}\left(\lambda^{0}\right)$ tem suporte em $(-(4-$ $\epsilon) \ln \kappa, \infty)$ e d $\hat{\sigma}_{\vec{\lambda}}^{b}\left(\lambda^{0}\right)$ em $(-(5-\epsilon) \ln \kappa, \infty)$. Z( $\left.\vec{p}\right)$ é uma função positiva e suave dada por

$$
\left(Z^{j}\right)^{-1}(\vec{p})=-\left.(2 \pi)^{2} e^{w^{j}} \frac{\partial \tilde{\Gamma}^{j}}{\partial \chi}\left(p_{0}=i \chi, \vec{p}\right)\right|_{\chi=w^{j}}
$$

onde $w^{m}\left(w^{b}\right)$ é a curva de dispersão para mésons (bárions) e $\tilde{\Gamma}^{j}\left(p^{0}, \vec{p}\right)$ é a transformada de Fourier de $\Gamma^{m}\left(\Gamma^{b}\right)$, a inversa convolutiva da função de dois pontos $G^{m}\left(G^{b}\right)$. 
Usando o comportamento a curtas distâncias de $\Gamma(x)$ (como fizemos no Teorema 4.2) obtemos

$$
\tilde{\Gamma}^{m}(p) \simeq 1-\kappa^{2}\left(e^{-i p_{0}}+e^{i p_{0}}\right)+\frac{1}{4} \kappa^{2} \sum_{k=1,2}\left(e^{-i p_{k}}+e^{i p_{k}}\right)+\mathcal{O}\left(\kappa^{4}\right) .
$$

Levando em $\partial \tilde{\Gamma}^{m} / \partial \chi$ temos

$$
\left.\frac{\partial \tilde{\Gamma}^{m}}{\partial \chi}\left(p_{0}=i \chi, \vec{p}\right)\right|_{\chi=w^{j}}=-\kappa^{2}\left(e^{w^{m}}-e^{-w^{m}}\right) \simeq-1
$$

Desta forma obtemos o resultado

$$
Z^{m}(\vec{p})=(2 \pi)^{-2} e^{-\omega^{m}(\vec{p})}+\mathcal{O}\left(\kappa^{4}\right)
$$

e de forma similar

$$
Z^{b}(\vec{p})=(2 \pi)^{-2} e^{-\omega^{b}(\vec{p})}+\mathcal{O}\left(\kappa^{4}\right) .
$$

Agora nós obtemos o lema que versa sobre a propriedade da medida $d \hat{\sigma}_{\vec{\lambda}}^{m}\left(\lambda^{0}\right)$. Este resultado abaixo é importante para controlarmos as aproximações feitas na análise do estado ligado nas seções 3.3, 3.4 e no Capítulo 4 (veja [33]).

Lema $5 \int_{-1}^{1} d \hat{\sigma}_{\vec{\lambda}}^{m}\left(\lambda^{0}\right)=\mathcal{O}\left(\kappa^{4}\right)$.

Prova do Lema 5: Usando a representação espectral para $G^{m}\left(x^{0}=1, \vec{x}\right)$ da eq. (3.4) e tomando a transformada de Fourier no espaço obtemos

$$
\begin{aligned}
G^{m}\left(x^{0}=1, \overrightarrow{0}\right)+\sum_{|\vec{x}|_{1} \neq 0} e^{-i \vec{q} \cdot \vec{x}} G\left(x^{0}=1, \vec{x}\right) & =(2 \pi)^{2} \int_{-1}^{1} d \sigma_{\vec{\lambda}}^{m}\left(\lambda^{0}\right) \\
& =(2 \pi)^{2} Z^{m}(\vec{p})+(2 \pi)^{2} \int_{-1}^{1} d \hat{\sigma}_{\vec{\lambda}}^{m}\left(\lambda^{0}\right)
\end{aligned}
$$

Usando o comportamento a curta distância de $G^{m}\left(x^{0}=1, \overrightarrow{0}\right)=\kappa^{2}+\mathcal{O}\left(\kappa^{4}\right)$ (Teorema 3.1 adaptado ao caso que estamos tratando aqui), $\left|G\left(x^{0}, \vec{x}\right)\right| \leq \kappa^{2\left(\left|x^{0}\right|+|\vec{x}|\right)}$ (observando que a cota obtida no Teorema 3.2 vale neste caso) obtemos que o termo $G^{m}\left(x^{0}=1, \overrightarrow{0}\right)$ é cancelado (até a ordem $\kappa^{2}$ ) com $(2 \pi)^{2} Z^{m}(\vec{p})=\kappa^{2}+\mathcal{O}\left(\kappa^{4}\right)$ (usando $w^{m}(\vec{p}) \simeq-2 \ln \kappa$ ) onde $Z^{m}(\vec{p})$ é dado pela eq. (3.7) e ainda $\left|G\left(x^{0}=1, \vec{x}\right)\right| \leq \kappa^{2} \kappa^{2|\vec{x}|_{1}} \leq \mathcal{O}\left(\kappa^{4}\right)$ para $|\vec{x}|_{1} \neq 0$.

De forma similar obtemos $\int_{-1}^{1} d \hat{\sigma}_{\vec{\lambda}}^{b}\left(\lambda^{0}\right)=\mathcal{O}\left(\kappa^{4}\right)$.

No subespaço gerado por duas partículas a combinação de dois mésons que iremos usar para analisar a existência do estado ligado é dada por

$$
M \equiv \Pi\left(1 / 2, \vec{x}_{1}\right) \Pi\left(1 / 2, \vec{x}_{2}\right) \text { e } L \equiv \Pi\left(1 / 2, \vec{x}_{3}\right) \Pi\left(1 / 2, \vec{x}_{4}\right)
$$

Desta forma, obtemos pela Proposição 1 o seguinte resultado, $x_{1}^{0}=x_{2}^{0}=u^{0}$ e $x_{3}^{0}=x_{4}^{0}=$ 
$v^{0}$

$$
\mathcal{G}_{M, L}(x) \equiv \begin{cases}\left\langle\mu\left(u^{0}, \vec{x}_{1}\right) \mu\left(u^{0}, \vec{x}_{2}\right) \Pi\left(v^{0}, \vec{x}_{3}+\vec{x}\right) \Pi\left(v^{0}, \vec{x}_{4}+\vec{x}\right)\right\rangle, & u_{0} \leq v_{0} \\ \left\langle\Pi\left(u^{0}, \vec{x}_{1}\right) \Pi\left(u^{0}, \vec{x}_{2}\right) \mu\left(v^{0}, \vec{x}_{3}+\vec{x}\right) \mu\left(v^{0}, \vec{x}_{4}+\vec{x}\right)\right\rangle^{*}, & u_{0}>v_{0}\end{cases}
$$

No Apêndice B, veremos que modificando a expressão acima por um fator multiplicativo para pontos coincidentes, da seguinte forma

$$
\mathcal{F}_{M, L}(x)=h\left(\vec{x}_{1}, \vec{x}_{2}\right) \mathcal{G}_{M, L}(x) h\left(\vec{x}_{3}, \vec{x}_{4}\right)
$$

com

$$
h\left(\vec{x}_{i}, \vec{x}_{j}\right)=\sqrt{3 / 2} \delta\left(x_{i}-x_{j}\right)+\left(1-\delta\left(x_{i}-x_{j}\right)\right),
$$

após subtrairmos o truncamento parcial $\left\langle\mu\left(u^{0}, \vec{x}_{1}\right) \mu\left(u^{0}, \vec{x}_{2}\right)\right\rangle\left\langle\Pi\left(v^{0}, \vec{x}_{3}+\vec{x}\right) \Pi\left(v^{0}, \vec{x}_{4}+\vec{x}\right)\right\rangle$, se $u_{0} \leq v_{0}$, e $\left\langle\Pi\left(u^{0}, \vec{x}_{1}\right) \Pi\left(u^{0}, \vec{x}_{2}\right)\right\rangle^{*}\left\langle\mu\left(v^{0}, \vec{x}_{3}+\vec{x}\right) \mu\left(v^{0}, \vec{x}_{4}+\vec{x}\right)\right\rangle^{*}$, se $u_{0}>v_{0}$, teremos um decaimento mais rápido na direção temporal do núcleo da equação de B-S para distância temporal de uma unidade. Em particular, desejamos um decaimento mais rápido que o decaimento de dois mésons, neste caso $\kappa^{4\left|x_{3}^{0}-x_{1}^{0}\right|}$. Com as modificações mencionadas acima, conforme mostrado no Apêndice B, obtemos $\kappa^{6\left|x_{3}^{0}-x_{1}^{0}\right|}$.

Para analisarmos a existência do estado ligado de um méson e um bárion, nós consideramos o subespaço de estados gerados por $\Pi\left(1 / 2, \vec{x}_{1}\right) \bar{\phi}\left(1 / 2, \vec{x}_{2}\right)$. No subespaço gerado pela combinação de um méson e um bárion definimos

$$
F \equiv \Pi\left(1 / 2, \vec{x}_{1}\right) \bar{\phi}\left(1 / 2, \vec{x}_{2}\right) \text { e } H \equiv \Pi\left(1 / 2, \vec{x}_{3}\right) \bar{\phi}\left(1 / 2, \vec{x}_{4}\right)
$$

Pela fórmula de F-K, para $x^{0} \neq 0$, temos

$$
\left(\Pi\left(1 / 2, \vec{u}_{1}\right) \bar{\phi}\left(1 / 2, \vec{u}_{2}\right), \check{T}_{0}^{\left|x^{0}\right|-1} \check{T}^{\vec{x}} \Pi\left(1 / 2, \vec{u}_{3}\right) \bar{\phi}\left(1 / 2, \vec{u}_{4}\right)\right)_{\mathcal{H}}=\mathcal{G}_{F, H}(x)
$$

$\operatorname{com} x=\left(x^{0}=v^{0}-u^{0}, \vec{x}\right) \in \mathbb{Z}^{3}$ e, para $u_{1}^{0}=u_{2}^{0}=u^{0}$ e $u_{3}^{0}=u_{4}^{0}=v^{0}$

$$
\begin{aligned}
\mathcal{G}_{F, H}(x)= & -\left\langle\mu\left(u_{1}\right) \phi\left(u_{2}\right) \Pi\left(u_{3}+\vec{x}\right) \bar{\phi}\left(u_{4}+\vec{x}\right)\right\rangle \chi_{u^{0} \leq v^{0}} \\
& +\left\langle\Pi\left(u_{1}\right) \bar{\phi}\left(u_{2}\right) \mu\left(u_{3}+\vec{x}\right) \phi\left(u_{4}+\vec{x}\right)\right\rangle^{*} \chi_{u^{0}>v^{0}}
\end{aligned}
$$

e nós notamos que, neste caso, o truncamento parcial é zero porque temos um número ímpar de campos simples $(\psi$ e $\bar{\psi})$ nas médias. A função de quatro pontos para o méson-anti-bárion é obtida a partir de $\mathcal{G}_{F, H}(x)$ pelo uso da simetria de conjugação de carga da mesma forma que fizemos para $G^{b}$. De agora em diante, restringimos nossa análise ao setor de paridade positiva, i.e., fazemos

$$
\Pi\left(1 / 2, \vec{u}_{1}\right) \bar{\phi}\left(1 / 2, \vec{u}_{2}\right) \mapsto \mathbb{P}_{+}\left[\Pi\left(1 / 2, \vec{u}_{1}\right) \bar{\phi}\left(1 / 2, \vec{u}_{2}\right)\right]
$$


e

$$
\Pi\left(1 / 2, \vec{u}_{1}\right) \bar{\phi}\left(1 / 2, \vec{u}_{2}\right) \mapsto \mathbb{P}_{+}\left[\Pi\left(1 / 2, \vec{u}_{1}\right) \bar{\phi}\left(1 / 2, \vec{u}_{2}\right)\right]
$$

onde $\mathbb{P}_{+} \equiv(1 / 2)(1+\mathbb{P}), \mathbb{P}$ é a simetria de paridade do Teorema 2 e $\mathbb{P}_{+}$é a projeção no subespaço de paridade $(+1)$. Desta forma obtemos

$$
\begin{aligned}
\mathcal{G}_{\mathbb{P}_{+} F, \mathbb{P}_{+} H}(x)= & -\left\langle\mathbb{P}_{+}\left[\mu\left(u_{1}\right) \phi\left(u_{2}\right)\right] \mathbb{P}_{+}\left[\Pi\left(u_{3}+\vec{x}\right) \bar{\phi}\left(u_{4}+\vec{x}\right)\right]\right\rangle \chi_{u^{0} \leq v^{0}} \\
& +\left\langle\mathbb{P}_{+}\left[\Pi\left(u_{1}\right) \bar{\phi}\left(u_{2}\right)\right] \mathbb{P}_{+}\left[\mu\left(u_{3}+\vec{x}\right) \phi\left(u_{4}+\vec{x}\right)\right]\right\rangle^{*} \chi_{u^{0}>v^{0}}
\end{aligned}
$$

Conforme veremos no Apêndice $\mathrm{B}$, teremos o decaimento $\kappa^{7\left|x_{3}^{0}-x_{1}^{0}\right|}$ para o núcleo de $K$ (depois de passarmos para coordenadas relativas e tomarmos a transformada de Fourier na variável $\tau)$, que é maior que o decaimento do méson-bárion que é $\kappa^{5\left|x_{3}^{0}-x_{1}^{0}\right|}$. Usando a simetria de paridade $\mathbb{P}$ e observando que os campos mesônicos e bariônicos, considerados aqui, satisfazem $\mathbb{P} \Pi(u)=-\Pi(\mathcal{P} u), \mathbb{P} \bar{\phi}(u)=-\bar{\phi}(\mathcal{P} u)$ e o mesmo para os outros campos compostos, $\mu$ e $\phi$, obtemos

$$
\begin{aligned}
\mathcal{G}_{\mathbb{P}_{+} F, \mathbb{P}_{+} H}(x)=\mathcal{G}_{\mathbb{P}_{+} F, H}(x)= & -\left\langle\mathbb{P}_{+}\left[\mu\left(u_{1}\right) \phi\left(u_{2}\right)\right] \Pi\left(u_{3}+\vec{x}\right) \bar{\phi}\left(u_{4}+\vec{x}\right)\right\rangle \chi_{u^{0} \leq v^{0}} \\
& +\left\langle\mathbb{P}_{+}\left[\Pi\left(u_{1}\right) \bar{\phi}\left(u_{2}\right)\right] \mu\left(u_{3}+\vec{x}\right) \phi\left(u_{4}+\vec{x}\right)\right\rangle^{*} \chi_{u^{0}>v^{0}} .
\end{aligned}
$$

Antes de continuarmos, apresentaremos a representação da equação de B-S em coordenadas relativas para a função de quatro mésons e a sua conexão com o espectro de energiamomento que usaremos para analisar o espectro abaixo do limiar de duas partículas mesônicas $(-4 \ln \kappa)$.

\subsection{A equação de Bethe-Salpeter na rede para o caso méson- méson}

Na seção anterior definimos $\mathcal{F}_{M, L}(x)$, e a conexão com o espectro de energia-momento é dada por sua transformada de Fourier $\tilde{\mathcal{F}}_{M, L}(k)$ pela Proposição 1. Desta forma, podemos relacionar singularidades em $k=\left(k^{0}, \overrightarrow{0}\right), k^{0}$ complexo, de $\tilde{\mathcal{F}}_{M, L}(k)$ com o espectro de energiamomento.

Para analisar $\tilde{\mathcal{F}}_{M, L}(k)$, simplificamos nossa notação renomeando as coordenadas na direção do tempo por inteiros, com $u_{i}^{0}-1 / 2=x_{i}^{0}, \vec{u}_{i}=\vec{x}_{i}, i=1, \ldots 4$, e assim definimos 
$D\left(x_{1}, x_{2}, x_{3}+\vec{x}, x_{4}+\vec{x}\right)$ onde

$$
\begin{aligned}
& D\left(x_{1}, x_{2}, x_{3}, x_{4}\right) \equiv \\
& \quad h\left(x_{1}, x_{2}\right)\left\langle\mu\left(x_{1}^{0}+1 / 2, \vec{x}_{1}\right) \mu\left(x_{2}^{0}+1 / 2, \vec{x}_{2}\right) ; \Pi\left(x_{3}^{0}+1 / 2, \vec{x}_{3}\right) \Pi\left(x_{4}^{0}+1 / 2, \vec{x}_{4}\right)\right\rangle_{T} h\left(x_{3}, x_{4}\right) \chi_{x_{2}^{0} \leq x_{3}^{0}} \\
& \quad+h\left(x_{1}, x_{2}\right)\left\langle\Pi\left(x_{1}^{0}+1 / 2, \vec{x}_{1}\right) \Pi\left(x_{2}^{0}+1 / 2, \vec{x}_{2}\right) ; \mu\left(x_{3}^{0}+1 / 2, \vec{x}_{3}\right) \mu\left(x_{4}^{0}+1 / 2, \vec{x}_{4}\right)\right\rangle_{T}^{*} h\left(x_{3}, x_{4}\right) \chi_{x_{2}^{0}>x_{3}^{0}}
\end{aligned}
$$

onde $h$ é dado pela eq. (3.11). Usando a invariância translacional do núcleo de $D$ passamos para coordenadas de diferença

$$
D\left(x_{1}, x_{2}, x_{3}+\vec{x}, x_{4}+\vec{x}\right)=D\left(0, x_{2}-x_{1}, x_{3}-x_{1}+\vec{x}, x_{4}-x_{1}+\vec{x}\right) .
$$

A seguir, usando a definição de coordenadas relativas do Apêndice C $\left(\xi=x_{2}-x_{1}, \eta=x_{4}-x_{3}\right.$ e $\tau=x_{3}-x_{2}$ ) obtemos

$$
\begin{aligned}
D\left(x_{1}, x_{2}, x_{3}+\vec{x}, x_{4}+\vec{x}\right) & =D\left(0, x_{2}-x_{1}=\xi, x_{3}-x_{1}=\xi+\tau+\vec{x}, x_{4}-x_{1}=\xi+\eta+\tau+\vec{x}\right) \\
& \equiv \bar{D}(\vec{\xi}, \vec{\eta}, \tau+\vec{x})
\end{aligned}
$$

e por um abuso de notação, deste ponto em diante omitiremos - em $\bar{O}$ sempre que escrevermos um dado operador $O$ em coordenadas relativas. Pela definição (3.13) e a eq. (3.14), tomando a transformada de Fourier na variável $x$ com variável conjugada $k$ obte$\operatorname{mos} \tilde{\mathcal{F}}_{M, L}(k)=e^{i \vec{k} \cdot \vec{\tau}} \hat{D}(\vec{\xi}, \vec{\eta}, k)$, onde $\hat{D}(\vec{\xi}, \vec{\eta}, k)=\sum_{\tau \in \mathbb{Z}^{3}} D(\vec{\xi}, \vec{\eta}, \tau) e^{-i k \cdot \tau}$. O objetivo de tudo isso é que singularidades de $\tilde{\mathcal{F}}_{M, L}(k)$, para $k=\left(k^{0}, \overrightarrow{0}\right), k^{0}$ complexo, que estão relacionadas ao espectro de energia-momento pela Proposição 1 , são as mesmas que as de $\hat{D}(\vec{\xi}, \vec{\eta}, k)$.

Para analisarmos $\hat{D}(\vec{\xi}, \vec{\eta}, k)$ consideramos a equação de B-S que, em termos de operadores é dada por

$$
D=D_{0}+D_{0} K D
$$

ou, equivalentemente, em termos de núcleos, na representação para tempos iguais,

$$
\begin{aligned}
& D\left(x_{1}, x_{2}, x_{3}, x_{4}\right)=D_{0}\left(x_{1}, x_{2}, x_{3}, x_{4}\right)+ \\
& \int D_{0}\left(x_{1}, x_{2}, y_{1}, y_{2}\right) K\left(y_{1}, y_{2}, y_{3}, y_{4}\right) D\left(y_{3}, y_{4}, x_{3}, x_{4}\right) \\
& \times \delta\left(y_{1}^{0}-y_{2}^{0}\right) \delta\left(y_{3}^{0}-y_{4}^{0}\right) d y_{1} d y_{2} d y_{3} d y_{4} ; x_{1}^{0}=x_{2}^{0}, x_{3}^{0}=x_{4}^{0},
\end{aligned}
$$

onde

$$
D_{0}\left(x_{1}, x_{2}, x_{3}, x_{4}\right)=G^{m}\left(x_{1}, x_{3}\right) G^{m}\left(x_{2}, x_{4}\right)+G^{m}\left(x_{1}, x_{4}\right) G^{m}\left(x_{2}, x_{3}\right) .
$$

$D_{0}$ é obtido partir de $D$ (sem os fatores $h$ ) pela aplicação "errônea" do teorema de Wick para os campos compostos $\mu$ e $\Pi$, e usamos notação do contínuo (i.e. o sinal de integração) para 
soma sobre pontos da rede. Nós observamos que como temos as simetrias

$$
D\left(x_{1}, x_{2}, x_{3}, x_{4}\right)=D\left(x_{2}, x_{1}, x_{3}, x_{4}\right)=D\left(x_{1}, x_{2}, x_{4}, x_{3}\right),
$$

tomamos $D, D_{0}$ e $K=D_{0}^{-1}-D^{-1}$ como sendo operadores matriciais agindo em $\ell_{2}^{s}(\mathcal{A})$, o subespaço simétrico de $\ell_{2}(\mathcal{A})$ gerado por vetores que são invariantes pela transformação $\left(x_{1}, x_{2}\right) \rightarrow\left(x_{2}, x_{1}\right)$, onde $\mathcal{A}=\left\{\left(x_{1}, x_{2}\right) \in \mathbb{Z}^{3} \times \mathbb{Z}^{3} / x_{1}^{0}=x_{2}^{0}\right\}$. Observamos ainda que, a restrição $x_{1}^{0}=x_{2}^{0}$ para os vetores de $A$ é imposta por estarmos considerando a equação de B-S na representação para tempos iguais. Em outras palavras, um vetor que pertence a $\ell_{2}^{s}(\mathcal{A})$ tem necessariamente a forma $c\left[f\left(x_{1}, x_{2}\right)+f\left(x_{2}, x_{1}\right)\right](c \in \mathbb{C})$ com $f\left(x_{1}, x_{2}\right) \in \ell_{2}(\mathcal{A})$. Definindo uma estimativa para a norma de um operador matricial $M(x, y)$ em $\ell_{2}(\mathcal{A})$ pelo Lema de Holmgren, observamos que $D_{0}$ é um operador limitado, pelo decaimento exponencial das funções de dois pontos (Teorema 3.2). Mais detalhes serão dados a seguir. Usando o fator $h$, dado na eq. (3.11), $D$ pode ser reescrito como $D=h\left(S_{4}^{T}+D_{0}\right) h$ onde $S_{4}$ é a função de correlação de quatro pontos truncada que tem decaimento em árvore. Nós definimos a inversa de $D\left(D_{0}\right)$ através da série de Neumann. Reescrevendo $D\left(D_{0}\right)$ como $D \equiv D_{0}+\delta D\left(D_{0} \equiv D_{0}^{(0)}+\delta D_{0}\right)$, de onde $\delta D \equiv D-D_{0}\left(\delta D_{0} \equiv D_{0}-D_{0}^{(0)}\right)$, para obter

$$
D^{-1}=\sum_{n=0}^{\infty}(-1)^{n}\left[D_{0}^{-1} \delta D\right]^{n} D_{0}^{-1},
$$

e

$$
D_{0}^{-1}=\sum_{n=0}^{\infty}(-1)^{n}\left[\left(D_{0}^{(0)}\right)^{-1} \delta D_{0}\right]^{n}\left(D_{0}^{(0)}\right)^{-1}
$$

onde $D_{0}^{(0)}$ é o termo de ordem $\kappa^{0}$ na expansão de $D_{0}$ em $\kappa$. Com isso, observamos que $\left\|\left|\delta D\|\|,\left\|\mid \delta D_{0}\right\| \| \leq \mathcal{O}\left(\kappa^{2}\right)\right.\right.$, onde \|\|$\bullet \|$ indica norma do operador agindo em $\ell_{2}^{s}(\mathcal{A})$. Desta forma, podemos garantir que $D$ e $D_{0}$ existem e a convergência da série de Neumann para $D^{-1}\left(D_{0}^{-1}\right)$ em (3.16) quando $0<\kappa<<1$. Nós agora exemplificamos a aplicação do Lema de Holmgren para mostrarmos que $\left\|\mid \delta D_{0}\right\| \| \leq \mathcal{O}\left(\kappa^{2}\right)$. De fato, para, $x_{1}^{0}=x_{2}^{0}$ e $x_{3}^{0}=x_{4}^{0}$ nós primeiro observamos que

$$
\delta D_{0}\left(x_{1}, x_{2}, x_{3}, x_{4}\right)=c \kappa^{2} \delta\left(x_{1}-x_{3}\right) \delta\left(x_{2}-x_{4}\right)+\delta D_{0}\left(1-\delta\left(x_{1}-x_{3}\right) \delta\left(x_{2}-x_{4}\right)\right)
$$

e nós temos

$$
\begin{aligned}
\delta D_{0}\left(x_{1}, x_{2}, x_{3}, x_{4}\right)= & G^{m}\left(x_{1}, x_{3}\right) G^{m}\left(x_{2}, x_{4}\right)+G^{m}\left(x_{1}, x_{4}\right) G^{m}\left(x_{2}, x_{3}\right) \\
& -\left(G^{m}\right)^{(0)}\left(x_{1}, x_{3}\right)\left(G^{m}\right)^{(0)}\left(x_{2}, x_{4}\right)-\left(G^{m}\right)^{(0)}\left(x_{1}, x_{4}\right)\left(G^{m}\right)^{(0)}\left(x_{2}, x_{3}\right) .
\end{aligned}
$$


Na expressão acima nós consideramos o termo

$$
\begin{aligned}
A \equiv & G^{m}\left(x_{1}, x_{3}\right) G^{m}\left(x_{2}, x_{4}\right)-\left(G^{m}\right)^{(0)}\left(x_{1}, x_{3}\right)\left(G^{m}\right)^{(0)}\left(x_{2}, x_{4}\right) \\
= & {\left[G^{m}\left(x_{1}, x_{3}\right)-\left(G^{m}\right)^{(0)}\left(x_{1}, x_{3}\right)+\left(G^{m}\right)^{(0)}\left(x_{1}, x_{3}\right)\right] } \\
& \times\left[G^{m}\left(x_{2}, x_{4}\right)-\left(G^{m}\right)^{(0)}\left(x_{2}, x_{4}\right)+\left(G^{m}\right)^{(0)}\left(x_{2}, x_{4}\right)\right] \\
& -\left(G^{m}\right)^{(0)}\left(x_{1}, x_{3}\right)\left(G^{m}\right)^{(0)}\left(x_{2}, x_{4}\right) \\
= & {\left[G^{m}\left(x_{1}, x_{3}\right)-\left(G^{m}\right)^{(0)}\left(x_{1}, x_{3}\right)\right]\left[G^{m}\left(x_{2}, x_{4}\right)-\left(G^{m}\right)^{(0)}\left(x_{2}, x_{4}\right)\right] } \\
& +\left[G^{m}\left(x_{1}, x_{3}\right)-\left(G^{m}\right)^{(0)}\left(x_{1}, x_{3}\right)\right]\left(G^{m}\right)^{(0)}\left(x_{2}, x_{4}\right) \\
& +\left[G^{m}\left(x_{2}, x_{4}\right)-\left(G^{m}\right)^{(0)}\left(x_{2}, x_{4}\right)\right]\left(G^{m}\right)^{(0)}\left(x_{1}, x_{3}\right) .
\end{aligned}
$$

Nós observamos agora que, pelo Teorema 3.2 (cuja estimativa permanece válida usando as definições deste capítulo),

$$
\left|G^{m}\left(x_{1}, x_{3}\right)-\left(G^{m}\right)^{(0)}\left(x_{1}, x_{3}\right)\right| \leq c_{1} \kappa^{2} \delta\left(x_{1}-x_{3}\right)+c_{2} \kappa^{2\left|x_{3}-x_{1}\right|_{1}}\left(1-\delta\left(x_{1}-x_{3}\right)\right)
$$

e o mesmo para $\left(x_{1}, x_{3}\right) \mapsto\left(x_{2}, x_{4}\right)$. Desta forma obtemos para a eq. (3.18)

$$
\begin{aligned}
\left|\delta D_{0}\left(x_{1}, x_{2}, x_{3}, x_{4}\right)\right|= & c \kappa^{2} \delta\left(x_{1}-x_{3}\right) \delta\left(x_{2}-x_{4}\right) \\
& +\left\{\left|c_{1} \kappa^{2} \delta\left(x_{1}-x_{3}\right)+c_{2} \kappa^{2\left|x_{3}-x_{1}\right|_{1}}\left(1-\delta\left(x_{1}-x_{3}\right)\right)\right|\right. \\
& \times\left|c_{1} \kappa^{2} \delta\left(x_{2}-x_{4}\right)+c_{2} \kappa^{2\left|x_{2}-x_{4}\right|_{1}}\left(1-\delta\left(x_{2}-x_{4}\right)\right)\right| \\
& +\left|c_{1} \kappa^{2} \delta\left(x_{1}-x_{3}\right)+c_{2} \kappa^{2\left|x_{3}-x_{1}\right|_{1}}\left(1-\delta\left(x_{1}-x_{3}\right)\right)\right| \\
& \times\left(G^{m}\right)^{(0)}\left(x_{2}, x_{4}\right)+\left|c_{1} \kappa^{2} \delta\left(x_{2}-x_{4}\right)+c_{2} \kappa^{2\left|x_{2}-x_{4}\right|_{1}}\left(1-\delta\left(x_{2}-x_{4}\right)\right)\right| \\
& \left.\times\left(G^{m}\right)^{(0)}\left(x_{1}, x_{3}\right)+\left[x_{3} \rightleftharpoons x_{4}\right]\right\}\left(1-\delta\left(x_{1}-x_{3}\right) \delta\left(x_{2}-x_{4}\right)\right)
\end{aligned}
$$

Nós agora podemos aplicar o Lema de Holmgren para a expressão resultante acima, i.e.

$\left\|\left|\delta D_{0}\right|\right\| \leq\left(\sup _{\left(x_{1}, x_{2}\right)} \sum_{\left(x_{3}, x_{4}\right)}\left|\delta D_{0}\left(x_{1}, x_{2}, x_{3}, x_{4}\right)\right|\right)^{1 / 2}\left(\sup _{\left(x_{3}, x_{4}\right)} \sum_{\left(x_{1}, x_{2}\right)}\left|\delta D_{0}\left(x_{1}, x_{2}, x_{3}, x_{4}\right)\right|\right)^{1 / 2} \leq \mathcal{O}\left(\kappa^{2}\right)$.

Nós agora, exemplificamos nosso método para obtermos a inversa de um dado operador para o caso $D_{0}^{-1}$. Para obtermos uma expressão explícita para $D_{0}^{-1}$, que será usada no cálculo da aproximação escada, reescrevemos $D_{0}$ da seguinte forma

$$
\begin{aligned}
D_{0}\left(x_{1}, x_{2}, x_{3}, x_{4}\right)= & D_{0}^{(0)}(0) \delta\left(x_{1}-x_{3}\right) \delta\left(x_{2}-x_{4}\right) \delta\left(x_{1}-x_{2}\right)+D_{0}^{(0)}\left(x_{1}, x_{2}, x_{3}, x_{4}\right) \\
& \times\left[1-\delta\left(x_{1}-x_{3}\right) \delta\left(x_{2}-x_{4}\right) \delta\left(x_{1}-x_{2}\right)\right]
\end{aligned}
$$


onde usamos a notação compacta $D_{0}^{(0)}(0) \equiv D_{0}\left(x_{1}=0, x_{2}=0, x_{3}=0, x_{4}=0\right)$ e, para pontos não-coincidentes, temos

$$
D_{0}^{(0)}\left(x_{1}, x_{2}, x_{3}, x_{4}\right)=\delta\left(x_{1}-x_{3}\right) \delta\left(x_{2}-x_{4}\right)+\delta\left(x_{1}-x_{4}\right) \delta\left(x_{2}-x_{3}\right)
$$

Como $D_{0}^{(0)}\left(x_{1}, x_{2}, x_{3}, x_{4}\right)$ age em $\ell_{2}^{s}(\mathcal{A})$ temos que

$$
D_{0}^{(0)}\left(x_{1}, x_{2}, x_{3}, x_{4}\right)=2 \delta\left(x_{1}-x_{3}\right) \delta\left(x_{2}-x_{4}\right)
$$

Podemos ver pela expressão (3.19) que $D_{0}^{-1}$ tem a forma

$$
D_{0}^{-1}\left(x_{1}, x_{2}, x_{3}, x_{4}\right)=A \delta\left(x_{1}-x_{3}\right) \delta\left(x_{2}-x_{4}\right) \delta\left(x_{1}-x_{2}\right)+B\left[1-\delta\left(x_{1}-x_{3}\right) \delta\left(x_{2}-x_{4}\right) \delta\left(x_{1}-x_{2}\right)\right]
$$

onde $A, B \in \mathbb{R}$. Usando a equação $D_{0} D_{0}^{-1}=1$ para os núcleos e a unicidade da inversa obtemos

$$
\begin{aligned}
D_{0}^{-1}\left(x_{1}, x_{2}, x_{3}, x_{4}\right)= & {\left[D_{0}^{(0)}(0)\right]^{-1} \delta\left(x_{1}-x_{3}\right) \delta\left(x_{2}-x_{4}\right) \delta\left(x_{1}-x_{2}\right)+2 \delta\left(x_{1}-x_{3}\right) \delta\left(x_{2}-x_{4}\right) } \\
& \times\left[1-\delta\left(x_{1}-x_{3}\right) \delta\left(x_{2}-x_{4}\right) \delta\left(x_{1}-x_{2}\right)\right] .
\end{aligned}
$$

Em coordenadas relativas, a equação de B-S na representação de tempos iguais fica expressa como (veja o Apêndice C)

$$
\hat{D}(\vec{\xi}, \vec{\eta}, k)=\hat{D}_{0}(\vec{\xi}, \vec{\eta}, k)+\int \hat{D}_{0}\left(\vec{\xi}, \vec{\xi}^{\prime}, k\right) \hat{K}\left(-\vec{\xi}^{\prime},-\vec{\eta}^{\prime}, k\right) \hat{D}\left(\vec{\eta}^{\prime}, \vec{\eta}, k\right) d \vec{\xi}^{\prime} d \vec{\eta}^{\prime}
$$

Com $k$ fixado, $\hat{D}(\vec{\xi}, \vec{\eta}, k)$, etc, é tomado como um operador matricial em $\ell_{2}\left(\mathbb{Z}^{2}\right)$ para $k=$ $\left(k^{0}, \vec{k}=\overrightarrow{0}\right)$ no subespaço par de $\ell_{2}\left(\mathbb{Z}^{2}\right)$. Conhecida a equação de B-S, passamos a sua solução.

O próximo passo consiste no cálculo da aproximação escada para $K$ e na resolução da equação de B-S para $K=L$.

\subsection{Ausência de estados ligados do tipo méson-méson, em $2+1$ dimensões, um sabor e matrizes de spin $2 \times 2$}

Nós agora obtemos o que chamamos de aproximação escada $L$ para $K$ (veja [13]). A aproximação escada corresponde à contribuição dominante do parâmetro de "hopping" $\kappa$ quando expandimos $D^{-1}$ e $D_{0}^{-1}$ em série de Neumann de acordo com as expressões (3.16) e (3.17), respectivamente. Nós usamos o superescrito $(n)$, para designar o termo de ordem $\kappa^{n}$. No nosso caso, $L$ é dado pela contribuição de ordem $\kappa^{2}$ de $D_{0}^{-1}\left(\delta D^{(2)}-\delta D_{0}^{(2)}\right) D_{0}^{-1}$. Conforme veremos a seguir, a contribuição de ordem $\kappa^{2}$ para $K$ vem se tomarmos o sítio de 
$\mu$ no ponto $x_{1}$ distante uma unidade do sítio de $\mu \Pi \Pi\left(x_{2}\right)\left(x_{2}=x_{3}=x_{4}\right)$ e similarmente para as outras três contribuições possíveis, $x_{i}$ distante de $x_{j}=x_{k}=x_{l}$ de uma unidade $(i=2,3,4$ e $i \neq j, k, l \in 1,2,3,4)$. Graficamente esta contribuição corresponde a, por exemplo,

$$
x _ { 1 } = 0 \longdiv { < } \epsilon e ^ { i } = x _ { 2 } = x _ { 3 } = x _ { 4 }
$$

Antes de determinarmos $L$ lembramos da seção anterior que

$$
D_{0}^{-1}\left(x_{1}, x_{2}, x_{3}, x_{4}\right)=\frac{1}{2} \delta\left(x_{1}-x_{3}\right) \delta\left(x_{2}-x_{4}\right) .
$$

Desta forma na expansão em série de Neumann temos,

$$
D_{0}^{-1} \delta D^{(2)} D_{0}^{-1}=\frac{1}{4} \delta D^{(2)} \text { e } D_{0}^{-1} \delta D_{0}^{(2)} D_{0}^{-1}=\frac{1}{4} \delta D_{0}^{(2)}
$$

e passamos à determinação de $\delta D^{(2)}$ e $\delta D_{0}^{(2)}$.

Para determinarmos $\delta D^{(2)}\left(\delta D_{0}^{(2)}\right)$ consideramos todas as contribuições para $\delta D\left(\delta D_{0}\right)$ até a ordem $\kappa^{2}$. Com uma barra (-) denotamos separação unitária (distância de uma unidade na rede) de um dado campo composto relativamente a outro, temos que considerar as seguintes configurações para $\delta D$ : (i) $\mu-\mu-\Pi-\Pi$, (ii) $\mu \Pi-\mu-\Pi$, (iii) $\Pi \Pi-\mu-\mu$, (iv) $\mu \mu-\Pi-\Pi$, (v) $\mu \Pi-\mu \Pi$, (vi) $\mu \mu-\Pi \Pi$, (vii) $\mu \mu \Pi-\Pi$ e (viii) $\mu \Pi \Pi-\mu$. Nós também notamos que os truncamentos $\langle\mu \mu\rangle\langle\Pi \Pi\rangle$ são pelo menos da ordem $\kappa^{4}$ para todos os casos listados e não contribuem na $\mathcal{O}\left(\kappa^{2}\right)$. Também, as configurações que podem contribuir para $\delta D_{0}$ na ordem $\kappa^{2}$ são (ii), (vii) e (viii).

Nós primeiro consideramos $\delta D^{(2)}$; os casos (i), (iii) e (iv) são zero já que temos ou uma média do tipo $\langle\mu\rangle$ e/ou $\langle\Pi\rangle$, que é zero pela simetria de paridade do Teorema 2, ou uma média do tipo $\langle\mu \mu\rangle$ ou $\langle\Pi \Pi\rangle$, que é zero devido a ausência de balanceamento nos campos fermiônicos (i.e. o número de $\psi_{\alpha, a}$ é distinto do número de $\bar{\psi}_{\alpha, a}$ na médias $\langle\mu \mu\rangle$ e $\langle\Pi \Pi\rangle$ ). A configuração (ii) é cancelada pela contribuição vinda de $\delta D_{0}^{(2)}$. Podemos ver que a configuração (vi) é zero da seguinte maneira, expandindo $\delta D^{(2)}$ em potências de $\kappa$ como fizemos na demonstração do Teorema 3 obtemos

$$
\frac{\kappa^{2}}{12}\left\langle\mu \mu \bar{\psi}_{\alpha_{1}, a_{1}} \psi_{\beta_{2}, a_{1}}(0)\right\rangle^{(0)}\left\langle\psi_{\beta_{1}, b_{1}} \bar{\psi}_{\alpha_{2}, b_{1}} \Pi \Pi\left(\epsilon e^{j}\right)\right\rangle^{(0)} \Gamma_{\alpha_{1} \beta_{1}}^{\epsilon e^{j}} \Gamma_{\alpha_{2} \beta_{2}}^{-\epsilon e^{j}}
$$

Na expressão acima, devemos ter $\alpha_{1}=\beta_{2}$ (usando a simetria de paridade do Teorema 2), mas pela ausência de balanceamento nos campos fermiônicos nas componentes de spin obtemos 
zero.

A configuração (v) é dada por, escrevendo explicitamente $\mu(0)$ e $\Pi(0)$ em termos dos campos fundamentais $\psi$ e $\bar{\psi}$

$$
\frac{\kappa^{2}}{12}\left\langle\psi_{-, a} \bar{\psi}_{+, a} \bar{\psi}_{-, b} \psi_{+, b} \bar{\psi}_{\alpha_{1}, a_{1}} \psi_{\beta_{2}, a_{1}}(0)\right\rangle^{(0)}\left\langle\psi_{\beta_{1}, a_{2}} \bar{\psi}_{\alpha_{2}, a_{2}} \mu \Pi\left(\epsilon e^{j}\right)\right\rangle^{(0)} \Gamma_{\alpha_{1} \beta_{1}}^{\epsilon e^{j}} \Gamma_{\alpha_{2} \beta_{2}}^{-\epsilon \epsilon}
$$

onde, relembrando, \langle\rangle$^{(0)}$ é a média \langle\rangle fazendo $\kappa=0$. Nós agora analisamos o primeiro fator da última expressão. Para $a=b$, pela saturação na cor (i.e. $\psi_{\alpha, a}(x) \psi_{\alpha, a}(x)=0$ e o mesmo para $\bar{\psi}$ ) obtemos $a_{1} \neq a$ e então $\alpha_{1}=\beta_{2}$. Usando a propriedade das matrizes $\Gamma$ dada pela eq. (2.26) obtemos zero para a eq. (3.21). Para $a \neq b$ o primeiro fator é zero pela ausência de balanceamento nas variáveis fermiônicas para $a_{1}=1,2,3$.

As configurações restantes são (vii) e (viii) para $\delta D$ e $\delta D_{0}$. Considerando $\delta D^{(2)}$ temos

$$
\delta D^{(2)}\left(e^{1}, 0,0,0\right)=\sqrt{\frac{3}{2}} \frac{1}{12}\left\langle\mu \mu \Pi \psi_{\alpha_{1}, a} \bar{\psi}_{\beta_{2}, a}(0)\right\rangle^{(0)}\left\langle\bar{\psi}_{\beta_{1}, b} \psi_{\alpha_{2}, b} \Pi\left(e^{1}\right)\right\rangle^{(0)} \Gamma_{\alpha_{1} \beta_{1}}^{e^{1}} \Gamma_{\alpha_{2} \beta_{2}}^{-e^{1}}
$$

onde o fator $\sqrt{3 / 2}$ vem da correção $h$ (veja a eq. (3.11)) para o decaimento de $K$. Pelo balanceamento nas variáveis fermiônicas devemos ter $\alpha_{1}=\beta_{1}=+$ e $\alpha_{2}=\beta_{2}=-$. Lembrando que $\Gamma_{--}=\Gamma_{++}=-1$ e usando o Teorema de Wick para $\langle\mu \mu \Pi \Pi(0)\rangle^{(0)}=4 / 3$ obtemos

$$
\delta D^{(2)}\left(e^{1}, 0,0,0\right)=\sqrt{\frac{3}{2}} \frac{1}{4}\langle\mu \mu \Pi \Pi(0)\rangle^{(0)}=\sqrt{\frac{3}{2}} \frac{1}{4} \frac{4}{3}=\frac{1}{2} \sqrt{\frac{2}{3}} .
$$

Para $\delta D_{0}^{(2)}$ temos

$$
\begin{aligned}
\delta D_{0}^{(2)}\left(e^{1}, 0,0,0\right)= & \left(G^{m}\right)^{(2)}\left(x_{1}=e^{1}, x_{3}=0\right)\left(G^{m}\right)^{(0)}\left(x_{2}=0, x_{4}=0\right) \\
& +\left(G^{m}\right)^{(2)}\left(x_{1}=e^{1}, x_{4}=0\right)\left(G^{m}\right)^{(0)}\left(x_{2}=0, x_{3}=0\right) \\
= & \frac{1}{6}\left\langle\mu \psi_{\alpha_{1}, a} \bar{\psi}_{\beta_{2}, a}\left(e^{1}\right)\right\rangle^{(0)}\left\langle\bar{\psi}_{\beta_{1}, b} \psi_{\alpha_{2}, b} \Pi(0)\right\rangle^{(0)} \Gamma_{\alpha_{1} \beta_{1}}^{e^{1}} \Gamma_{\alpha_{2} \beta_{2}}^{-e^{1}}=\frac{1}{2} .
\end{aligned}
$$

As outras contribuições, correspondentes a $\delta D_{0}^{(2)}\left(\epsilon e^{i}, 0,0,0\right)(\epsilon= \pm, i=2$ e $\epsilon=-$, $i=1) ; \delta D_{0}^{(2)}\left(0, \epsilon e^{i}, 0,0\right)(\epsilon= \pm \mathrm{e} i=1,2) ; \delta D_{0}^{(2)}\left(0,0, \epsilon e^{i}, 0\right)(\epsilon= \pm \mathrm{e} i=1,2) ; \delta D_{0}^{(2)}\left(0,0,0, \epsilon e^{i}\right)$ $(\epsilon= \pm$ e $i=1,2)$ são iguais a $\delta D\left(e^{1}, 0,0,0\right)$, usando simetria de paridade e reversão no tempo do Teorema 2 e as simetrias da eq. (3.15). O mesmo resultado vale para $\delta D^{(2)}$.

Desta forma, usando as eqs. (3.22) e (3.23), obtemos para $L=K^{(2)} \kappa^{2}$, com $c^{\prime \prime}(\kappa)=$ 


$$
\begin{aligned}
& (\sqrt{2 / 3}-1) \kappa^{2} / 8 \\
& \quad L\left(x_{1}, x_{2}, x_{3}, x_{4}\right)=\frac{1}{4}\left[\delta D^{(2)}\left(e^{1}, 0,0,0\right)-\delta D_{0}^{(2)}\left(e^{1}, 0,0,0\right)\right] \kappa^{2} \\
& \quad \times \sum_{\epsilon, j}\left[\delta\left(x_{1}-x_{2}-\epsilon e^{j}\right) \delta\left(x_{3}-x_{2}\right) \delta\left(x_{4}-x_{3}\right)+\delta\left(x_{3}-x_{1}\right) \delta\left(x_{4}-x_{1}\right) \delta\left(x_{2}-x_{1}-\epsilon e^{j}\right)\right. \\
& \left.\quad+\delta\left(x_{2}-x_{1}\right) \delta\left(x_{3}-x_{1}-\epsilon e^{j}\right) \delta\left(x_{4}-x_{1}\right)+\delta\left(x_{2}-x_{1}\right) \delta\left(x_{3}-x_{1}\right) \delta\left(x_{4}-x_{1}-\epsilon e^{j}\right)\right] \\
& \quad=c^{\prime \prime}(\kappa) \sum_{\epsilon, j}\left[\delta\left(x_{1}-x_{2}-\epsilon e^{j}\right) \delta\left(x_{3}-x_{2}\right) \delta\left(x_{4}-x_{3}\right)+\delta\left(x_{3}-x_{1}\right) \delta\left(x_{4}-x_{1}\right) \delta\left(x_{2}-x_{1}-\epsilon e^{j}\right)\right. \\
& \left.\quad+\delta\left(x_{2}-x_{1}\right) \delta\left(x_{3}-x_{1}-\epsilon e^{j}\right) \delta\left(x_{4}-x_{1}\right)+\delta\left(x_{2}-x_{1}\right) \delta\left(x_{3}-x_{1}\right) \delta\left(x_{4}-x_{1}-\epsilon e^{j}\right)\right]
\end{aligned}
$$

Em coordenadas relativas (veja a eq. (C.1)), obtemos

$$
L(\vec{\xi}, \vec{\eta})=2 c^{\prime \prime}(\kappa) \sum_{j, \epsilon}\left[\delta(\vec{\eta}) \delta\left(\vec{\xi}-\epsilon e^{j}\right)+\delta\left(\vec{\eta}-\epsilon e^{j}\right) \delta(\vec{\xi})\right]
$$

Nós agora obtemos uma representação para $\hat{D}_{0}$ que será usada para analisar a possível existência do estado ligado. Considerando $\hat{D}_{0}$ em coordenadas relativas, tomamos a transformada de Fourier na variável $\tau$, com variável conjugada $k$ e fazemos $k=\left(k^{0}, \overrightarrow{0}\right)$, para obtermos

$$
\begin{aligned}
\hat{D}_{0}\left(\vec{\xi}, \vec{\eta}, k^{0}\right)= & \sum_{\vec{\tau}} G^{m}(0, \vec{\tau}+\vec{\xi}) G^{m}(0, \vec{\tau}+\vec{\eta}) \\
& +\sum_{\tau \mid \tau^{0} \neq 0} e^{-i \tau^{0} k^{0}} G^{m}(0, \tau+\vec{\xi}) G^{m}(0, \tau+\vec{\eta})+[\vec{\xi} \rightarrow 0, \vec{\eta} \rightarrow \vec{\xi}+\vec{\eta}] .
\end{aligned}
$$

Seja $A$ uma notação para o segundo termo do lado direito da expressão anterior. Usando a representação espectral dada pela eq. (3.4), temos que

$$
\begin{aligned}
A= & \sum_{\tau \mid \tau^{0} \neq 0} e^{-i k^{0} \tau^{0}}\left[\int_{-1}^{1} \int_{\mathbf{T}^{2}}\left(\lambda^{0}\right)^{\left|\tau^{0}\right|-1} e^{i \vec{p} \cdot(\vec{\tau}+\vec{\xi})} d \sigma_{\vec{p}}^{m}\left(\lambda^{0}\right) d \vec{p}\right] \\
& \times\left[\int_{-1}^{1} \int_{\mathbf{T}^{2}}\left(\lambda^{0}\right)^{\left|\tau^{0}\right|-1} e^{i \vec{p} \cdot(\vec{\tau}+\vec{\eta})} d \sigma_{\vec{q}}^{m}\left(\nu^{0}\right) d \vec{q}\right] d \tau^{0} d \vec{\tau} .
\end{aligned}
$$

Fazendo a soma na variável $\tau$ (usando a fórmula de Poisson, i.e. $\quad \sum_{\vec{\tau}} e^{i \vec{p} . \vec{\tau}}=(2 \pi)^{2} \delta(\vec{p})$ ), integrando na variável $\vec{q}$, obtemos

$$
\begin{aligned}
A & =(2 \pi)^{2} \int_{-1}^{1} \int_{-1}^{1} \sum_{\tau^{0}} e^{-i k^{0} \tau^{0}}\left(\lambda^{0} \nu^{0}\right)^{\left|\tau^{0}\right|-1} \delta(\vec{p}+\vec{q}) e^{i \vec{p} \cdot \vec{\xi}} e^{i \vec{q} \cdot \vec{\eta}} d \sigma_{\vec{p}}^{m}\left(\lambda^{0}\right) d \vec{p} d \sigma_{\vec{q}}^{m}\left(\nu^{0}\right) d \vec{q} \\
& =(2 \pi)^{2} \int_{-1}^{1} \int_{-1}^{1} f\left(k^{0}, \lambda^{0} \nu^{0}\right) e^{i \vec{p} \cdot(\vec{\xi}-\vec{\eta})} d \sigma_{\vec{p}}^{m}\left(\lambda^{0}\right) d \sigma_{-\vec{p}}^{m}\left(\nu^{0}\right) d \vec{p}
\end{aligned}
$$


onde $f(x, y)$ é o mesmo da Proposição 1. Usando, nesta expressão, $\sigma_{\vec{p}}=\sigma_{-\vec{p}}$, (obtido usando a simetria da transformada de Fourier da função de dois pontos para mésons $\tilde{G}^{m}\left(p^{0}, \vec{p}\right)=$ $\left.\tilde{G}^{m}\left(p^{0},-\vec{p}\right)\right)$, e

$$
e^{i \vec{p} \cdot(\vec{\xi}-\vec{\eta})}=\cos (\vec{p} \cdot \vec{\xi}) \cos (\vec{p} \cdot \vec{\eta})+\operatorname{sen}(\vec{p} \cdot \vec{\xi}) \operatorname{sen}(\vec{p} \cdot \vec{\eta})+i[\operatorname{sen}(\vec{p} \cdot \vec{\xi}) \cos (\vec{p} \cdot \vec{\eta})-\operatorname{sen}(\vec{p} \cdot \vec{\eta}) \cos (\vec{p} \cdot \vec{\xi})]
$$

obtemos

$$
\begin{aligned}
A= & (2 \pi)^{2} \int_{-1}^{1} \int_{-1}^{1} f\left(k^{0}, \lambda^{0} \nu^{0}\right)[\cos (\vec{p} \cdot \vec{\xi}) \cos (\vec{p} \cdot \vec{\eta})+\operatorname{sen}(\vec{p} \cdot \vec{\xi}) \operatorname{sen}(\vec{p} \cdot \vec{\eta})] \\
& +i[\operatorname{sen}(\vec{p} \cdot \vec{\xi}) \cos (\vec{p} \cdot \vec{\eta})-\operatorname{sen}(\vec{p} \cdot \vec{\eta}) \cos (\vec{p} \cdot \vec{\xi})] d \sigma_{\vec{p}}^{m}\left(\lambda^{0}\right) d \sigma_{\vec{p}}^{m}\left(\nu^{0}\right) d \vec{p}
\end{aligned}
$$

Temos também o termo correspondente vindo de $[\vec{\xi} \rightarrow 0, \vec{\eta} \rightarrow \vec{\xi}+\vec{\eta}]$ na eq. (3.25), que chamaremos de $B$

$$
\begin{aligned}
B= & (2 \pi)^{2} \int_{-1}^{1} \int_{-1}^{1} f\left(k^{0}, \lambda^{0} \nu^{0}\right)[\cos (\vec{p} \cdot \vec{\xi}) \cos (\vec{p} \cdot \vec{\eta})-\operatorname{sen}(\vec{p} \cdot \vec{\xi}) \operatorname{sen}(\vec{p} \cdot \vec{\eta})] \\
& -i[\operatorname{sen}(\vec{p} \cdot \vec{\xi}) \cos (\vec{p} \cdot \vec{\eta})+\operatorname{sen}(\vec{p} \cdot \vec{\eta}) \cos (\vec{p} \cdot \vec{\xi})] d \sigma_{\vec{p}}^{m}\left(\lambda^{0}\right) d \sigma_{\vec{p}}^{m}\left(\nu^{0}\right) d \vec{p}
\end{aligned}
$$

Nós agora consideramos o primeiro termo do lado direito da eq. (3.25) e seu correspondente em $[\vec{\xi} \rightarrow \overrightarrow{0}, \vec{\eta} \rightarrow \vec{\xi}+\vec{\eta}]$

$$
\sum_{\vec{\tau}} G^{m}(0, \vec{\tau}+\vec{\xi}) G^{m}(0, \vec{\tau}+\vec{\eta})+[\vec{\xi} \rightarrow 0, \vec{\eta} \rightarrow \vec{\xi}+\vec{\eta}]
$$

Para o primeiro termo da eq. (3.27) observamos que $\left(\tilde{G}^{m}(p)=\sum_{x} e^{-i p x} G^{m}(x)\right)$

$$
\sum_{\vec{\tau}}\left[(2 \pi)^{-3} \int \tilde{G}^{m}(p) e^{i \vec{p} \cdot(\vec{\tau}+\vec{\xi})} d p\right]\left[(2 \pi)^{-3} \int \tilde{G}^{m}(q) e^{i \vec{q} \cdot(\vec{\tau}+\vec{\eta})} d q\right]
$$

Fazendo a soma na variável $\vec{\tau}$ e a integral na variável $q$ obtemos

$$
(2 \pi)^{-4} \int \tilde{G}^{m}(p) \tilde{G}^{m}(q) \delta(\vec{p}+\vec{q}) e^{i \vec{p} \cdot(\vec{\xi}-\vec{\eta})} d p d q=(2 \pi)^{-4} \int \tilde{G}^{m}(p) \tilde{G}^{m}\left(q^{0},-\vec{p}\right) e^{i \vec{p} \cdot(\vec{\xi}-\vec{\eta})} d q^{0} d p^{0} d \vec{p}
$$

Levando em conta o segundo termo na eq. (3.27) obtemos

$$
\begin{aligned}
& (2 \pi)^{-4} \int \tilde{G}^{m}(p) \tilde{G}^{m}\left(q^{0},-\vec{p}\right)\left[e^{i \vec{p} \cdot(\vec{\xi}-\vec{\eta})}+e^{-i \vec{p} \cdot(\vec{\xi}+\vec{\eta})}\right] d q^{0} d p^{0} d \vec{p} \\
& =2(2 \pi)^{-3} \int \tilde{G}^{m}(p) \tilde{G}^{m}\left(q^{0},-\vec{p}\right)[\cos (\vec{p} \cdot \vec{\xi}) \cos (\vec{p} \cdot \vec{\eta})+i \cos (\vec{p} \cdot \vec{\xi}) \operatorname{sen}(\vec{p} \cdot \vec{\eta})] d q^{0} d p^{0} d \vec{p}
\end{aligned}
$$


Lembrando que,

$$
\int \tilde{G}^{m}\left(p^{0}, \vec{p}\right) d p^{0}=\sum_{x^{0}, \vec{x}} \int e^{-i p^{0} x^{0}} e^{-i \vec{p} \cdot \vec{x}} G^{m}(x) d p^{0}
$$

a integração em $p^{0}$ fornece $2 \pi \delta\left(x^{0}\right)$ e a soma em $x^{0}$

$$
\int \tilde{G}^{m}\left(p^{0}, \vec{p}\right) d p^{0}=2 \pi \sum_{\vec{x}} e^{-i \vec{p} \cdot \vec{x}} G^{m}\left(x^{0}=0, \vec{x}\right) \equiv 2 \pi \tilde{G}^{m}(\vec{p}) .
$$

Desta forma, considerando os termos $A, B$ e a eq. (3.28) obtemos a seguinte expressão para $\hat{D}_{0}\left(\vec{\xi}, \vec{\eta}, k^{0}\right)$

$$
\hat{D}_{0}\left(\vec{\xi}, \vec{\eta}, k^{0}\right)=2 \int_{\mathbf{T}^{2}} M\left(\vec{p}, k^{0}\right)[\cos (\vec{p} \cdot \vec{\xi}) \cos (\vec{p} \cdot \vec{\eta})-i \operatorname{sen}(\vec{p} \cdot \vec{\eta}) \cos (\vec{p} \cdot \vec{\xi})] d \vec{p}
$$

onde $M\left(\vec{p}, k^{0}\right)$ é dado por

$$
M\left(\vec{p}, k^{0}\right)=(2 \pi)^{-2}\left(\tilde{G}^{m}\right)^{2}(\vec{p})+(2 \pi)^{2} \int_{-1}^{1} \int_{-1}^{1} f\left(k^{0}, \lambda^{0} \nu^{0}\right) d \sigma_{\vec{p}}^{m}\left(\lambda_{0}\right) d \sigma_{\vec{p}}^{m}\left(\nu_{0}\right) .
$$

Usando as simetrias $\hat{D}\left(\vec{\xi}, \vec{\eta}, k^{0}\right)=\hat{D}\left(-\vec{\xi}, \vec{\eta}, k^{0}\right)=\hat{D}\left(\vec{\xi},-\vec{\eta}, k^{0}\right)$ obtemos

$$
\hat{D}_{0}\left(\vec{\xi}, \vec{\eta}, k^{0}\right)=2 \int_{\mathbf{T}^{2}} M\left(\vec{p}, k^{0}\right) \cos (\vec{p} \cdot \vec{\xi}) \cos (\vec{p} \cdot \vec{\eta}) d \vec{p}
$$

Para investigarmos a possível existência de um estado ligado buscamos as possíveis singularidades de $\hat{D}$, para $k^{0}$ no eixo imaginário, perto do limiar de dois mésons $(-4 \ln \kappa)$. Isto corresponde a tomar $k^{0}=i \chi=2 m^{m}-\bar{\epsilon}$ na equação para $\hat{D}\left(\vec{\xi}, \vec{\eta}, k^{0}\right)$. A contribuição dominante para $\hat{D}_{0}$ vem se desprezarmos a parte independente de $k^{0}$, i.e. $(2 \pi)^{-2}\left(\tilde{G}^{m}\right)^{2}(\vec{p})$ e mantermos somente o produto de contribuições de um méson, i.e. $d \sigma_{\vec{p}}^{m}\left(\lambda^{0}\right) \simeq Z^{m}(\vec{p}) \delta\left(\lambda^{0}-e^{-\omega^{m}(\vec{p})}\right) d \lambda^{0}$ pela estimativa do Lema 5 (veja [33]). Desta forma, obtemos

$$
\hat{D}_{0}\left(\vec{\xi}, \vec{\eta}, k^{0}\right) \simeq 2(2 \pi)^{2} \int_{\mathbf{T}^{2}} \frac{\left(Z^{m}\right)^{2}(\vec{p})}{e^{i k^{0}-e^{-2 w^{m}(\vec{p})}}} \cos \vec{p} \cdot \vec{\xi} \cos \vec{p} \cdot \vec{\eta} d \vec{p}
$$

Fazendo $k^{0}=i \chi=i\left(2 m^{m}-\bar{\epsilon}\right)$, e usando a expressão para $Z^{m}(\vec{p})$ da eq. (3.7), tal que $\bar{\epsilon}>0$ é a energia de ligação para o méson-méson, temos se $\bar{\epsilon}<<1$

$$
\hat{D}_{0}\left(\vec{\xi}, \vec{\eta}, k^{0}\right) \simeq(2 \pi)^{-2} \int_{\mathbf{T}^{2}} \frac{\cos \vec{p} \cdot \vec{\xi} \cos \vec{p} \cdot \vec{\eta}}{2 c^{m} \kappa^{2}[-\tilde{\Delta}(\vec{p})]+\bar{\epsilon}} d \vec{p}
$$

No contexto da aproximação dada pela eq. (3.31) na equação de B-S, fazemos as identificações $a=\kappa^{2} / 2, \lambda=[1-\sqrt{2 / 3}] \kappa^{2} / 4, V=\sum_{j, \epsilon}\left[\delta(\vec{\xi}) \delta\left(\vec{\eta}-\epsilon e^{j}\right)+\delta\left(\vec{\xi}-\epsilon e^{j}\right) \delta(\vec{\eta})\right]$ e $z=-\bar{\epsilon} / 2$ na equação 
de Schrödinger na forma resolvente,

$$
(H-z)^{-1}=\left(H_{0}-z\right)^{-1}-\lambda\left(H_{0}-z\right)^{-1} V(H-z)^{-1},
$$

onde $H=H_{0}+\lambda V, H_{0}=-a \Delta / 2\left[-\Delta\right.$ é o Laplaciano na rede em $\left.\ell_{2}\left(\mathbb{Z}^{2}\right)\right]$ e $V$ é um potencial não-local agindo no subespaço par de $\ell_{2}\left(\mathbb{Z}^{2}\right)$.

A equação de B-S na aproximação escada é escrita como, deste ponto em diante, por simplicidade omitiremos da $k^{0}$ da notação para $\hat{D}\left(\vec{\xi}, \vec{\eta}, k^{0}\right)$, etc

$$
\hat{D}(\vec{\xi}, \vec{\eta})=\hat{D}_{0}(\vec{\xi}, \vec{\eta})+2 c^{\prime \prime}(\kappa) \sum_{j, \epsilon}\left[\hat{D}_{0}(\vec{\xi}, 0) \hat{D}\left(\epsilon e^{j}, \vec{\eta}\right)+\hat{D}_{0}\left(\vec{\xi}, \epsilon e^{j}\right) \hat{D}(0, \vec{\eta})\right]
$$

e agora obtemos sua solução. A eq. (3.32) pode ser simplificada ainda mais lembrando que, como $\hat{D}(\vec{\xi}, \vec{\eta})$, etc são operadores que agem no subespaço simétrico de $\ell_{2}\left(\mathbb{Z}^{2}\right)$, temos $\hat{D}(\vec{\xi}, \vec{\eta})=\hat{D}(-\vec{\xi}, \vec{\eta})=\hat{D}(\vec{\xi},-\vec{\eta})$, etc. Assim, obtemos, com $c^{\prime}(\kappa) \equiv 4 c^{\prime \prime}(\kappa)$

$$
\hat{D}(\vec{\xi}, \vec{\eta})=\hat{D}_{0}(\vec{\xi}, \vec{\eta})+c^{\prime}(\kappa) \sum_{j, \epsilon}\left[\hat{D}_{0}(\vec{\xi}, 0) \hat{D}\left(e^{j}, \vec{\eta}\right)+\hat{D}_{0}\left(\vec{\xi}, e^{j}\right) \hat{D}(0, \vec{\eta})\right]
$$

Primeiramente, re-escalonamos a eq. (3.33) por um fator $\kappa^{2} / 2$, desta forma $\hat{D} \mapsto\left(\kappa^{2} / 2\right) \hat{D} \equiv \hat{R}$, $\hat{D}_{0} \mapsto\left(\kappa^{2} / 2\right) \hat{D}_{0} \equiv \hat{R}_{0}$ e $c^{\prime}(\kappa) \mapsto 2 c^{\prime}(\kappa) / \kappa^{2} \equiv c(\kappa)$. Resolvendo a eq. (3.33) re-escalonada para as variáveis $\hat{R}(0, \vec{\eta}), \hat{R}\left(e^{j}, \vec{\eta}\right)(j=1,2)$ vemos que as singularidades possíveis de $\hat{R}$, que são as mesmas de $\hat{D}$, são dadas pelos zeros do determinante da matriz $3 \times 3 \mathrm{~W}$, com $c \equiv c(\kappa)=2 c^{\prime}(\kappa) / \kappa^{2}, r_{00}=\hat{R}_{0}(0,0), r_{0 i}=\hat{R}_{0}\left(0, e^{i}\right)$ e $r_{i j}=\hat{R}_{0}\left(e^{i}, e^{j}\right)(i, j=1,2)$,

$$
W \equiv\left(\begin{array}{rrr}
1-2 c\left(r_{01}+r_{02}\right) & -2 c r_{00} & -2 c r_{00} \\
-2 c\left(r_{11}+r_{12}\right) & 1-2 c r_{10} & -2 c r_{10} \\
-2 c\left(r_{11}+r_{12}\right) & -2 c r_{20} & 1-2 c r_{20}
\end{array}\right)
$$

Usando a simetria $r_{01}=r_{02}$, obtida reescrevendo $\hat{D}_{0}\left(0, e^{1}\right)$ da seguinte forma, usando a eq. $(3.25)$

$$
\hat{D}_{0}\left(0, e^{1}\right)=2 \sum_{\tau} e^{i k^{0} \tau^{0}} G^{m}(0, \tau) G^{m}\left(0, \tau+e^{1}\right)
$$

e observando que, pela simetria de rotação de $\pi / 2$ em relação ao eixo $e^{3}$ do Teorema 2 ,

$$
G^{m}(0, \tau)=\langle\mu(0) \Pi(\tau)\rangle=\left\langle\mu(0) \Pi\left(r_{3}(\tau)\right)\right\rangle
$$

Da mesma forma,

$$
G^{m}\left(0, \tau+e^{1}\right)=\left\langle\mu(0) \Pi\left(\tau+e^{1}\right)\right\rangle=\left\langle\mu(0) \Pi\left(r_{3}(\tau)+e^{2}\right)\right\rangle .
$$


Assim, lembrando que estamos somando em $\vec{\tau}$ temos

$$
\begin{aligned}
\hat{D}_{0}\left(0, e^{1}\right) & =2 \sum_{\tau} e^{i k^{0} \tau^{0}} G^{m}\left(0, r_{3}(\tau)\right) G^{m}\left(0, r_{3}(\tau)+e^{2}\right) \\
& =2 \sum_{\tau} e^{i k^{0} \tau^{0}} G^{m}(0, \tau) G^{m}\left(0, \tau+e^{2}\right)=\hat{D}_{0}\left(0, e^{2}\right)
\end{aligned}
$$

e o resultado segue observando que $r_{01}=\hat{R}_{0}\left(0, e^{1}\right)=\hat{R}\left(0, e^{2}\right)=r_{02}$. De forma similar as relações $r_{12}=r_{21}$ e $r_{11}=r_{22}$ são obtidas. Finalmente, usando estas relações, podemos reescrever det $W$ da seguinte forma

$$
\operatorname{det} W=\left|\begin{array}{rrr}
1-4 c r_{01} & -2 c r_{00} & -2 c r_{00} \\
-2 c\left(r_{11}+r_{12}\right) & 1-2 c r_{10} & -2 c r_{10} \\
-2 c\left(r_{21}+r_{22}\right) & -2 c r_{10} & 1-2 c r_{10}
\end{array}\right|
$$

Calculando este determinante usando a expansão de Laplace para a terceira coluna, obtemos

$$
\operatorname{det} W=\left|\begin{array}{rr}
1-4 c r_{01} & -4 c r_{00} \\
-2 c\left(r_{11}+r_{12}\right) & 1-4 c r_{01}
\end{array}\right| \text {. }
$$

Para obtermos os zeros de det $W$, usamos as seguintes relações:

$$
\begin{gathered}
r_{01}=-\frac{1}{2}+\frac{1}{2}(2+b) r_{00}, \\
r_{11}+r_{12}=(2+b) r_{01} .
\end{gathered}
$$

que podem ser verificadas reescrevendo $r_{00}, r_{01}, r_{11}$ e $r_{12}$ da seguinte forma

$$
\begin{gathered}
r_{00}=\frac{1}{(2 \pi)^{2}} \int_{\mathbf{T}^{2}} \frac{1}{B} d \vec{p}, \\
r_{01}=\frac{1}{(2 \pi)^{2}} \int_{\mathbf{T}^{2}} \frac{\cos p_{1}}{B} d \vec{p}, \\
r_{11}=\frac{1}{(2 \pi)^{2}} \int_{\mathbf{T}^{2}} \frac{\cos ^{2} p_{1}}{B} d \vec{p}, \\
r_{12}=\frac{1}{(2 \pi)^{2}} \int_{\mathbf{T}^{2}} \frac{\cos p_{1} \cos p_{2}}{B} d \vec{p}
\end{gathered}
$$


onde usamos a notação compacta $B=(2+b)-\cos p_{1}-\cos p_{2}$. Desta forma, temos para $r_{01}$

$$
\begin{aligned}
r_{01} & =\frac{1}{(2 \pi)^{2}} \frac{1}{2} \int_{\mathbf{T}^{2}} \frac{\cos p_{1}+\cos p_{2}}{B} d \vec{p} \\
& =\frac{1}{(2 \pi)^{2}} \frac{1}{2} \int_{\mathbf{T}^{2}} \frac{\cos p_{1}+\cos p_{2}-(2+b)+(2+b)}{B} d \vec{p} \\
& =-\frac{1}{2}+\frac{1}{2}(2+b) r_{00} .
\end{aligned}
$$

Para obtermos (3.37), usamos a expressão

$$
\frac{1}{(2 \pi)^{2}} \int_{\mathbf{T}^{2}} \frac{B^{2}}{B} d \vec{p}=\frac{1}{(2 \pi)^{2}} \int_{\mathbf{T}^{2}} B d \vec{p}=\frac{1}{(2 \pi)^{2}} \int_{\mathbf{T}^{2}}\left[(2+b)-\cos p_{1}-\cos p_{2}\right] d \vec{p}=(2+b) .
$$

Por outro lado, usando a identidade

$$
B^{2}=\left[\cos p_{1}+\cos p_{2}-(2+b)\right]^{2}=\left(\cos p_{1}+\cos p_{2}\right)^{2}-2(2+b)\left(\cos p_{1}+\cos p_{2}\right)+(2+b)^{2},
$$

temos a seguinte expressão

$$
\frac{1}{(2 \pi)^{2}} \int_{\mathbf{T}^{2}} \frac{B^{2}}{B} d \vec{p}=r_{3}-4(2+b) r_{10}+(2+b)^{2} r_{00}
$$

onde $r_{3}$ é definido por

$$
\begin{aligned}
r_{3} & \equiv \frac{1}{(2 \pi)^{2}} \int_{\mathbf{T}^{2}} \frac{\left(\cos p_{1}+\cos p_{2}\right)^{2}}{B} d \vec{p} \\
& =\frac{2}{(2 \pi)^{2}} \int_{\mathbf{T}^{2}} \frac{\cos ^{2} p_{1}+\cos p_{1} \cos p_{2}}{B} d \vec{p} \\
& =2\left(r_{11}+r_{12}\right) .
\end{aligned}
$$

Combinando (3.42) e (3.44) obtemos

$$
(2+b)=r_{3}-4(2+b) r_{10}+(2+b)^{2} r_{00}
$$

Em (3.46) usando (3.36) e (3.45) obtemos (3.37).

Retornando a eq. (3.35) e usando as relações (3.36) e (3.37), a condição para o estado ligado fica, onde $\alpha=(1-\sqrt{2 / 3}) / 2$,

$$
\begin{aligned}
\operatorname{det} W & =\left(1+4 c r_{01}\right)-8 c^{2} r_{00}\left(r_{11}+r_{12}\right) \\
& =(1-\alpha)^{2}+4 \alpha(1-\alpha)(2+b) r_{00}=0
\end{aligned}
$$


de onde obtemos a condição para a existência do estado ligado

$$
(1-\alpha)^{2}+4 \alpha(1-\alpha)(2+b) r_{00}=0
$$

Como $r_{00}$ é positivo, a equação (3.47) adimite solução somente quando $\alpha<0$ ou $\alpha>1$. No nosso caso, como $0<\alpha=(1-\sqrt{2 / 3}) / 2<1$ não existe estado ligado do tipo méson-méson.

\subsection{Ausência de estados ligados do tipo méson-bárion, em $2+1$ dimensões, um sabor e matrizes de spin $2 \times 2$}

A função de quatro pontos no caso de méson-bárion é dada por

$$
\begin{aligned}
& \mathcal{D}\left(x_{1}, x_{2}, x_{3}, x_{4}\right) \equiv \\
& \quad-\left\langle P_{+}\left[\mu\left(x_{1}^{0}+1 / 2, \vec{x}_{1}\right) \phi\left(x_{2}^{0}+1 / 2, \vec{x}_{2}\right)\right] \Pi\left(x_{3}^{0}+1 / 2, \vec{x}_{3}\right) \bar{\phi}\left(x_{4}^{0}+1 / 2, \vec{x}_{4}\right)\right\rangle \chi_{x_{2}^{0} \leq x_{3}^{0}} \\
& \left.\quad+P_{+}\left[\Pi\left(x_{1}^{0}+1 / 2, \vec{x}_{1}\right) \bar{\phi}\left(x_{2}^{0}+1 / 2, \vec{x}_{2}\right)\right] \mu\left(x_{3}^{0}+1 / 2, \vec{x}_{3}\right) \phi\left(x_{4}^{0}+1 / 2, \vec{x}_{4}\right)\right\rangle^{*} \chi_{x_{2}^{0}>x_{3}^{0}} .
\end{aligned}
$$

A conexão de $\tilde{\mathcal{G}}_{\mathbb{P}_{+} F, H}(k) \operatorname{com} \hat{\mathcal{D}}(\vec{\xi}, \vec{\eta}, k)$ no presente caso é feita seguindo os passos da Seção 3.2 e nós aqui omitiremos os detalhes.

A equação de B-S em termos de núcleos, na representação para tempos iguais, é dada por

$$
\begin{aligned}
& \mathcal{D}\left(x_{1}, x_{2}, x_{3}, x_{4}\right)=\mathcal{D}_{0}\left(x_{1}, x_{2}, x_{3}, x_{4}\right)+ \\
& \int \mathcal{D}_{0}\left(x_{1}, x_{2}, y_{1}, y_{2}\right) \mathcal{K}\left(y_{1}, y_{2}, y_{3}, y_{4}\right) \mathcal{D}\left(y_{3}, y_{4}, x_{3}, x_{4}\right) \times \\
& \delta\left(y_{1}^{0}-y_{2}^{0}\right) \delta\left(y_{3}^{0}-y_{4}^{0}\right) d y_{1} d y_{2} d y_{3} d y_{4} ; x_{1}^{0}=x_{2}^{0}, x_{3}^{0}=x_{4}^{0},
\end{aligned}
$$

neste caso $\mathcal{D}_{0}\left(x_{1}, x_{2}, x_{3}, x_{4}\right)=(1 / 2)\left[G^{m}\left(x_{1}-x_{3}\right) G^{b}\left(x_{2}-x_{4}\right)+G^{m}\left(\mathcal{P} x_{1}-x_{3}\right) G^{b}\left(\mathcal{P} x_{2}-x_{4}\right)\right]$. Observando que

$$
\mathcal{D}\left(x_{1}, x_{2}, x_{3}, x_{4}\right)=\mathcal{D}\left(-x_{1},-x_{2}, x_{3}, x_{4}\right)=\mathcal{D}\left(x_{1}, x_{2},-x_{3},-x_{4}\right) .
$$

Tomamos $\mathcal{D}, \mathcal{D}_{0}$ e $\mathcal{K}=\mathcal{D}_{0}^{-1}-\mathcal{D}^{-1}$ como operadores matriciais agindo em $\ell_{2}^{r}(A)$, o subespaço de $\ell_{2}(A)$ gerado por vetores que são invariantes pela reflexão no espaço $\left(x_{1}, x_{2}\right) \rightarrow\left(\mathcal{P} x_{1}, \mathcal{P} x_{2}\right)$, onde $A=\left\{\left(x_{1}, x_{2}\right) \in \mathbb{Z}^{3} \times \mathbb{Z}^{3} / x_{1}^{0}=x_{2}^{0}, \vec{x}_{1} \neq \vec{x}_{2}\right\}$. Em outras palavras, um vetor que pertence a $\ell_{2}^{r}(A)$ tem necessariamente a forma $c\left[f\left(x_{1}, x_{2}\right)+f\left(\mathcal{P} x_{1}, \mathcal{P} x_{2}\right)\right](c \in \mathbb{C}) \operatorname{com} f\left(x_{1}, x_{2}\right) \in \ell_{2}(A)$. Podemos mostrar usando o Lema de Holmgren que estes oeradores são limitados. Notamos ainda que, a restrição $\vec{x}_{1} \neq \vec{x}_{2}$ é imposta para descartamos as contribuições identicamente nulas na função de quatro pontos para o méson bárion, i.e., quando $x_{1}=x_{2}=x$ temos $\mu(x) \phi(x)=0$ ou quando $x_{3}=x_{4}=x$ segue que $\Pi(x) \bar{\phi}(x)=0$. Em termos das coordenadas relativas $(\vec{\xi}, \vec{\eta}, \tau)$ e tomando a transformada de Fourier na variável $\tau$, a equação de B-S fica 
como a eq. (3.20). $\hat{\mathcal{D}}(\vec{\xi}, \vec{\eta}, k)$, etc, é tomado como um operador matricial em $\ell_{2}(B)$, onde $B=\mathbb{Z}^{2} \backslash\{0\}$; para $k=\left(k^{0}, \vec{k}=\overrightarrow{0}\right)$ no subespaço par de $\ell_{2}(B)$.

Nós agora obtemos a aproximação escada $\mathcal{L}$ para $\mathcal{K}$ (see [13]). No nosso caso, $\mathcal{L}$ é dado pela contribuição de $\mathcal{O}\left(\kappa^{3}\right)$ para $\mathcal{D}_{0}^{-1} \mathcal{D}^{T} \mathcal{D}_{0}^{-1}$, o primeiro termo não-nulo na série de Neumann para $\mathcal{K}=\mathcal{D}_{0}^{-1}-\left[\mathcal{D}_{0}+\mathcal{D}^{T}\right]^{-1}$, onde $\mathcal{D}^{T}=\mathcal{D}-\mathcal{D}_{0}$ é a função truncada de quatro-pontos para o méson-bárion. A contribuição de $\mathcal{O}\left(\kappa^{2}\right)$ para $\mathcal{K}$ vem se tomarmos o mesmo sítio para $\phi(\Pi)$ e $\bar{\phi}$ $(\mu)$. Notamos que, esta é a única contribuição permitida nesta ordem. Se considerarmos, por exemplo, $x_{1}=x_{4}=0, x_{2}=x_{3}=x(|x|=1)$ teríamos zero pela ausência de balanceamento de campos fermiônicos nas médias. Outra possibilidade é dada pela contribuição

$$
\frac{1}{3}\left(\frac{\kappa}{2}\right)^{2}\left\langle\mu \Pi \bar{\psi}_{\alpha_{1}, a_{1}} \psi_{\beta_{2}, a_{1}}(0)\right\rangle^{(0)}\left\langle\psi_{\beta_{1}, a_{2}} \bar{\psi}_{\alpha_{2}, a_{2}} \phi \bar{\phi}\left(e^{j}\right)\right\rangle^{(0)} \Gamma_{\alpha_{1} \beta_{1}}^{e^{1}} \Gamma_{\alpha_{2} \beta_{2}}^{-e^{1}}
$$

onde, relembrando, \langle\rangle$^{(0)}$ é a média \langle\rangle $\operatorname{com} \kappa=0$. Nós agora analisamos o primeiro fator da última expressão. Seguindo a análise que fizemos para o caso méson-méson na Seção 3.3, em particular a contribuição (3.21) vemos que este termo é zero. Nós então obtemos zero para a contribuição de $\mathcal{O}\left(\kappa^{2}\right)$ para $\mathcal{K}$ então, excluindo uma interpretação de troca de mésons entre dois pares de partículas separados por uma unidade na rede. Usando a fórmula $\mathcal{I}_{3}$ da eq. (A.11), obtemos que a contribuição de $\mathcal{O}\left(\kappa^{3}\right)$, é local e dada por $x_{1}=x_{4}=0, x_{2}=x_{3}=x$ $(|x|=1)$ conectadas por três elos sobrepostos com a mesma orientação. Diagramaticamente temos

$$
x_{1}=x_{4}=0 \longrightarrow
$$

Expandindo $\mathcal{D}^{T}$ em potências de $\kappa$ obtemos

$-\frac{1}{36}\left(\frac{\kappa}{2}\right)^{3}\left\langle\mu \bar{\phi} \psi_{\beta_{1}, b_{1}} \psi_{\beta_{2}, b_{2}} \psi_{\beta_{3}, b_{3}}(0)\right\rangle^{(0)}\left\langle\bar{\psi}_{\alpha_{1}, a_{1}} \bar{\psi}_{\alpha_{2}, a_{2}} \bar{\psi}_{\alpha_{3}, a_{3}} \Pi \phi\left(e^{1}\right)\right\rangle^{(0)} \Gamma_{\alpha_{1} \beta_{1}}^{-e^{1}} \Gamma_{\alpha_{2} \beta_{2}}^{-e^{1}} \Gamma_{\alpha_{3} \beta_{3}}^{-e^{1}} \epsilon_{a_{1} a_{2} a_{3}} \epsilon_{b_{1} b_{2} b_{3}}$,

Pela eq. (3.48) vemos que a contribuição $\mathcal{O}\left(\kappa^{3}\right)$ para $\mathcal{D}$ vem do cálculo de contribuições de troca de três-férmions (e três-anti-férmions) entre pares de partículas do tipo mésonbárion tal que nós não podemos identificá-las com um bárion (relembrando, um campo bariônico é dado por $\left.\phi_{-}(u)=\psi_{-, 1}(u) \psi_{-, 2}(u) \psi_{-, 3}(u)\right)$ devido ao índice de spin (nós obtemos e.g. $\left.\psi_{+, 1}(u) \psi_{-, 2}(u) \psi_{-, 3}(u)\right)$. Usando o Teorema de Wick para a eq. (3.48) nós obtemos, com $c^{\prime}(\kappa)=-\frac{3}{16} \kappa^{3}$,

$\mathcal{L}\left(x_{1}, x_{2}, x_{3}, x_{4}\right)=c^{\prime}(\kappa) \sum_{j=1,2 ; \epsilon= \pm 1} \delta\left(x_{2}-x_{1}-\epsilon e^{j}\right)\left[\delta\left(x_{4}-x_{1}\right) \delta\left(x_{3}-x_{2}\right)+\delta\left(x_{4}+x_{1}\right) \delta\left(x_{3}+x_{2}\right)\right]$ 
e, como $\mathcal{L}$ age em $\ell_{2}^{r}(A)$, nós temos

$$
\mathcal{L}\left(x_{1}, x_{2}, x_{3}, x_{4}\right)=2 c^{\prime}(\kappa) \sum_{j=1,2 ; \epsilon= \pm 1} \delta\left(x_{2}-x_{1}-\epsilon e^{j}\right) \delta\left(x_{4}-x_{1}\right) \delta\left(x_{3}-x_{2}\right)
$$

e então, em coordenadas relativas, tomando a transformada de Fourier na variável $\tau$ com variável conjugada $k$,

$$
\hat{\mathcal{L}}\left(\vec{\xi}, \vec{\eta}, k^{0}\right)=2 c^{\prime}(\kappa) \sum_{j, \epsilon} \delta(\vec{\xi}-\vec{\eta}) \delta\left(\vec{\xi}-\epsilon e^{j}\right)
$$

onde usamos a notação abreviada $k^{0}$ para $k=\left(k^{0}, \vec{k}=\overrightarrow{0}\right)$, que omitiremos na seqüência.

A equação de B-S na aproximação escada é dada por, omitindo o índice $k^{0}$ da expressão para os operadores $\hat{D}$, etc, como fizemos na seção anterior,

$$
\hat{\mathcal{D}}(\vec{\xi}, \vec{\eta})=\hat{\mathcal{D}}_{0}(\vec{\xi}, \vec{\eta})+2 c^{\prime}(\kappa) \sum_{j, \epsilon} \hat{\mathcal{D}}_{0}\left(\vec{\xi}, \epsilon e^{j}\right) \hat{\mathcal{D}}\left(\epsilon e^{j}, \vec{\eta}\right)
$$

e nós agora obtemos sua solução. Seguindo a Seção 3.3 , usando as $\operatorname{simetrias} \hat{\mathcal{D}}_{0}(\vec{\xi}, \vec{\eta})=$ $\hat{\mathcal{D}}_{0}(-\vec{\xi}, \vec{\eta})=\hat{\mathcal{D}}_{0}(\vec{\xi},-\vec{\eta})$ e o mesmo para $\hat{\mathcal{D}}_{0}$, resolvemos a eq. (3.49) para as variáveis $\hat{\mathcal{D}}\left(e^{j}, \vec{\eta}\right)$ $(j=1,2)$ para obtermos $\left(\operatorname{com} c(\kappa)=4 c^{\prime}(\kappa)\right)$

$$
\hat{\mathcal{D}}(\vec{\xi}, \vec{\eta})=\hat{\mathcal{D}}_{0}(\vec{\xi}, \vec{\eta})+c(\kappa) \sum_{j k} \hat{\mathcal{D}}_{0}\left(\vec{\xi}, e^{j}\right) \frac{\mathcal{M}_{j k}}{W} \hat{\mathcal{D}}_{0}\left(e^{k}, \vec{\eta}\right)
$$

onde $\mathcal{M}$ denota a matriz $2 \times 2$ com entradas $\mathcal{M}_{11}=\mathcal{M}_{22}=1-c(\kappa) \hat{\mathcal{D}}_{0}\left(e^{1}, e^{1}\right), \mathcal{M}_{12}=$ $\mathcal{M}_{21}=-c(\kappa) \hat{\mathcal{D}}_{0}\left(e^{1}, e^{2}\right)$. Nós vemos que as únicas singularidades possíveis de $\hat{\mathcal{D}}(\vec{\xi}, \vec{\eta})$ para $k^{0}$ imaginário, e abaixo do limiar do méson-bárion $(-5 \ln \kappa)$, ocorrem como zeros de $W\left(k^{0}\right) \equiv$ $\operatorname{det} \mathcal{M}$.

Nós agora obtemos uma representação para $\hat{\mathcal{D}}_{0}$ que será usada para analisarmos a existência do estado ligado. Mantendo somente o produto de contribuições de um méson e um bárion para $\hat{\mathcal{D}}_{0}\left(\vec{\xi}, \vec{\eta}, k^{0}\right)$ obtemos, como o procedimento usado para chegarmos na eq. (3.30)

$$
\hat{\mathcal{D}}_{0}\left(\vec{\xi}, \vec{\eta}, k^{0}\right) \simeq \int_{\mathbf{T}^{2}} \mathcal{M}\left(\vec{p}, k^{0}\right)(\cos \vec{p} \cdot \vec{\xi} \cos \vec{p} \cdot \vec{\eta}+\operatorname{sen} \vec{p} \cdot \vec{\xi} \operatorname{sen} \vec{p} \cdot \vec{\eta}) d \vec{p}
$$

onde

$$
\mathcal{M}\left(\vec{p}, k^{0}\right)=(2 \pi)^{-2} \tilde{G}^{m}(\vec{p}) \tilde{G}^{b}(\vec{p})+(2 \pi)^{2} \int_{-1}^{1} \int_{-1}^{1} f\left(k^{0}, \lambda^{0} \nu^{0}\right) d \sigma_{\vec{p}}^{m}\left(\lambda_{0}\right) d \sigma_{\vec{p}}^{b}\left(\nu_{0}\right)
$$

com $f(x, y)$ o mesmo da Proposição 1.

Mantendo somente o produto de contribuições de um méson e um bárion para $\hat{\mathcal{D}}_{0}\left(\vec{\xi}, \vec{\eta}, k^{0}\right)$; desprezando a contribuição independente de $k^{0}$, i.e. $(2 \pi)^{-2} \tilde{G}^{m}(\vec{p}) \tilde{G}^{b}(\vec{p})$; fazendo $k^{0}=i \chi=$ 
$i\left(m_{\kappa}^{m}+m_{\kappa}^{b}-\bar{\epsilon}\right)$ tal que $\bar{\epsilon}>0$ é a energia de ligação do méson-bárion, temos a contribuição dominante $(\bar{\epsilon}<<1)$

$$
\hat{\mathcal{D}}_{0}\left(\vec{\xi}, \vec{\eta}, k^{0}\right) \simeq(2 \pi)^{-2} \int_{\mathbf{T}^{2}} \frac{\cos \vec{p} \cdot \vec{\xi} \cos \vec{p} \cdot \vec{\eta}}{\left(c^{m} \kappa^{2}+c^{b} \kappa^{3}\right)[-\tilde{\Delta}(\vec{p})]+\bar{\epsilon}} d \vec{p}
$$

Para determinarmos o estado ligado devemos analisar os zeros de

$$
W\left(k^{0}\right)=1-c(\kappa)\left[\hat{\mathcal{D}}_{0}\left(e^{1}, e^{1}\right) \mp \hat{\mathcal{D}}_{0}\left(e^{1}, e^{2}\right)\right] .
$$

Notando que $\left(c^{m} \kappa^{2}+c^{b} \kappa^{3}\right)[-\tilde{\Delta}(\vec{p})]+\bar{\epsilon}>0$ podemos usar a desigualdade de Cauchy-Schwarz para mostrar que $\left|\hat{\mathcal{D}}_{0}\left(e^{1}, e^{2}, k^{0}=i \chi\right)\right| \leq \hat{\mathcal{D}}_{0}\left(e^{1}, e^{1}, k^{0}=i \chi\right)$ e desta forma excluímos estados ligados do espectro, já que, no nosso caso obtemos $W\left(k^{0}\right) \geq 1$.

No contexto das aproximações acima, fazemos as seguintes identificações $a=c^{m} \kappa^{2}+$ $c^{b} \kappa^{3}, \lambda=\frac{3}{8} \kappa^{3}, V=\sum_{j, \epsilon} \delta(\vec{\xi}-\vec{\eta}) \delta\left(\vec{\xi}-\epsilon e^{j}\right)$ e $z=-\bar{\epsilon}$ na equação resolvente de Schrödinger, $(H-z)^{-1}=\left(H_{0}-z\right)^{-1}-\lambda\left(H_{0}-z\right)^{-1} V(H-z)^{-1}$, onde $H=H_{0}+\lambda V, H_{0}=-a \Delta / 2[-\Delta$ é o laplaciano em $\left.\ell_{2}\left(\mathbb{Z}^{2}\right)\right]$ e $V$ é um potencial local agindo em $\ell_{2}\left(\mathbb{Z}^{2} \backslash\{0\}\right)$. Desta forma um $\lambda$ positivo corresponde a um potencial repulsivo. 


\section{Capítulo 4}

\section{Um estado ligado méson-méson em $(2+1)$ dimensões, dois sabores e matrizes de Pauli $2 \times 2$}

Neste capítulo, seguindo [15], ainda no regime de acoplamento forte e na aproximação escada, apresentamos a análise do espectro de energia-momento para estados ligados no setor mesônico em $(2+1)$ dimensões, dois sabores de quarks (caso com isospin) e matrizes de spin $2 \times 2$. Nós usamos aqui, o análogo da ação dada pela eq. (1.2) com as adaptações necessárias para dois sabores, ou seja,

$$
\begin{aligned}
S(\psi, \bar{\psi}, g)= & \frac{\kappa}{2} \sum \bar{\psi}_{\alpha, a, f}(u) \Gamma_{\alpha \beta}^{\epsilon e^{\rho}}\left(g_{u, u+\epsilon e^{\rho}}\right)_{a b} \psi_{\beta, b, f}\left(u+\epsilon e^{\rho}\right) \\
& +\sum_{u \in \mathbb{Z}_{o}^{d+1}} \bar{\psi}_{\alpha, a, f}(u) M_{\alpha \beta} \psi_{\beta, a, f}(u)-\frac{1}{g_{0}^{2}} \sum_{p} \chi\left(g_{p}\right) .
\end{aligned}
$$

onde aqui temos a soma sobre o índice de sabor (isospin) $f=1,2$. A ação (4.1) tem as mesmas simetrias da ação (1.2) e, desta forma, os resultados a respeito de simetrias do Teorema 2 podem ser aplicados também aqui. Começamos este capítulo apresentando os campos mesônicos e introduzindo as modificações necessárias para o caso de dois sabores na função de dois pontos, para a qual vemos que os resultados anteriores envolvendo massas e curvas de dispersão dos mésons continuam válidos até ordem $\kappa^{2}$, ao menos.

Além de aumentar o volume do espaço de configurações para os quarks, o quê faz enfraquecer a repulsão de Pauli, com a adição de dois sabores, a ação (4.1) adquire uma simetria $U(2)$ global no setor de isospin. Nós restringimos nossa atenção ao setor de Isospin total zero, que tem uma menor componente $z$ de isospin, e desta forma uma menor repulsão de Pauli, aumentando as chances das partículas se ligarem. Na segunda seção, apresentamos uma descrição detalhada da simetria global $U(2)$ agindo no grau de liberdade de isospin ou 
sabor. Na terceira seção, apresentamos a equação de B-S na aproximação escada. Na analogia com a equação resolvente de Schrödinger obtemos que a aproximação escada corresponde a um potencial com dois tipos de contribuições: um potencial dependente da energia, local e de alcance zero e um potencial local de alcance espacial um, relacionado a troca de quasi-mésons. Resolvendo a equação de B-S na aproximação escada obtemos um estado ligado com energia de ligação $\bar{b} \kappa^{2} \equiv 0,02359 \kappa^{2}$. Após um estudo detalhado, verificamos que o mecanismo de atração dominante corresponde a um efeito de correlação relacionado a integral de calibre de quatro campos [veja a eq. (A.12)].

Todos os cálculos numéricos deste capítulo foram feitos usando o pacote Maple 9.03 pela Waterloo Maple Corporation.

Os resultados deste capítulo foram publicados em [16].

\subsection{A função de dois pontos e quatro pontos para mésons em $2+1$ dimensões, dois sabores e matrizes de spin $2 \times 2$}

Começamos introduzindo os campos mesônicos, invariantes por transformação de calibre, $\Pi_{l}, l=1,2,3,4$ (veja o capítulo anterior para a comparação com o caso de um sabor), por

$$
\Pi_{l}(u)= \begin{cases}\frac{1}{\sqrt{6}}\left[\bar{\psi}_{-, a,+}(u) \psi_{+, a,+}(u)+\bar{\psi}_{-, a,-}(u) \psi_{+, a,-}(u)\right] & ; l=1 \\ \frac{1}{\sqrt{3}} \bar{\psi}_{-, a,+}(u) \psi_{+, a,-}(u) & ; l=2 \\ \frac{1}{\sqrt{3}} \bar{\psi}_{-, a,-}(u) \psi_{+, a,+}(u) & ; l=3 \\ \frac{1}{\sqrt{6}}\left[\bar{\psi}_{-, a,+}(u) \psi_{+, a,+}(u)-\bar{\psi}_{-, a,-}(u) \psi_{+, a,-}(u)\right] & ; l=4\end{cases}
$$

e os campos auxiliares $\mu_{l}$

$$
\mu_{l}(u)= \begin{cases}\frac{1}{\sqrt{6}}\left[\psi_{-, a,+}(u) \bar{\psi}_{+, a,+}(u)+\psi_{-, a,-}(u) \bar{\psi}_{+, a,-}(u)\right] & ; l=1 \\ \frac{1}{\sqrt{3}} \psi_{-, a,+}(u) \bar{\psi}_{+, a,-}(u) & ; l=2 \\ \frac{1}{\sqrt{3}} \psi_{-, a,-}(u) \bar{\psi}_{+, a,+}(u) & ; l=3 \\ \frac{1}{\sqrt{6}}\left[\psi_{-, a,+}(u) \bar{\psi}_{+, a,+}(u)-\psi_{-, a,-}(u) \bar{\psi}_{+, a,-}(u)\right] & ; l=4\end{cases}
$$

A ação da simetria conjugação de carga $\mathcal{C}$ nos campos $\Pi_{l}$ é dada por $\mathcal{C} \Pi_{1,4}=-\Pi_{1,4}$ e $\mathcal{C} \Pi_{2,3}=$ $-\Pi_{3,2}$, e o mesmo para os campos auxiliares $\mu_{l}$. Pela simetria de paridade ou conjugação de carga do Teorema 2, temos

$$
\left\langle\Pi_{l}(u)\right\rangle=0,\left\langle\mu_{l}(u)\right\rangle=0 \quad ; \quad l=1,2,3,4 .
$$


Notamos que, como no caso de um sabor, na definição de $\Pi_{l}\left(\mu_{l}\right)$, o índice de spin nos campos básicos $\psi$ é $+(-)$ e é $-(+)$ para o campo de anti-quark $\bar{\psi}$. Também, conforme vimos no capítulo anterior $\Pi_{l}$ tem a interpretação de criação de mésons.

A motivação para as definições (4.2) e (4.3) se relacionam com a simetria $\mathrm{U}(2)$ de isospin da ação (4.1). A relação destes estados com os estados de isospin usuais, i.e. com isospin total $I$ e com componente, $z, I_{z}$ é feita na Seção 4.2. Conforme veremos, os campos compostos $\Pi_{l=1,2,3,4}$ podem ser identificados com os estados $\left(I, I_{z}\right)=(0,0),(1,1),(1,-1),(1,0)$, respectivamente.

Pela fórmula de F-K da Proposição 1 , para $x^{0} \neq 0$ em $\mathbb{Z}$ e tomando $\check{T}^{\vec{x}} \equiv \check{T}_{1}^{x^{1}} \check{T}_{2}^{x^{2}}$, temos $(k, l=1,2,3,4)$

$$
\left(\Pi_{k}\left(1 / 2, \vec{u}_{1}\right), \check{T}_{0}^{\left|x^{0}\right|-1} \check{T}^{\vec{x}} \Pi_{l}\left(1 / 2, \vec{u}_{2}\right)\right)_{\mathcal{H}} \equiv G_{k l}(x),
$$

onde, para $x=\left(x^{0}=u_{2}^{0}-u_{1}^{0}, \vec{x}\right) \in \mathbb{Z}^{3}$, temos $G_{k l}(x) \equiv G_{k l}\left(u_{1}, u_{2}+\vec{x}\right)$ e somos levados a definir a função de dois pontos associada

$$
\begin{aligned}
G_{k l}\left(u_{1}, u_{2}\right) & =\left\langle\mu_{k}\left(u_{1}\right) ; \Pi_{l}\left(u_{2}\right)\right\rangle_{T} \chi_{u_{1}^{0} \leq u_{2}^{0}}+\left\langle\Pi_{k}\left(u_{1}\right) ; \mu_{l}\left(u_{2}\right)\right\rangle_{T}^{*} \chi_{u_{1}^{0}>u_{2}^{0}} \\
& =\left\langle\mu_{k}\left(u_{1}\right) \Pi_{l}\left(u_{2}\right)\right\rangle \chi_{u_{1}^{0} \leq u_{2}^{0}}+\left\langle\Pi_{k}\left(u_{1}\right) \mu_{l}\left(u_{2}\right)\right\rangle^{*} \chi_{u_{1}^{0}>u_{2}^{0}}
\end{aligned}
$$

onde para obtermos a última igualdade usamos a simetria de paridade do Teorema 2, para obtermos $\left\langle\mu_{k}\right\rangle=\left\langle\Pi_{k}\right\rangle=0$.

Para o estado de duas partículas obtido pelo acoplamento de dois estados de isospin total zero, tomamos a combinação trivial de coeficientes de Clebsch-Gordan (C-G), $\operatorname{com} x_{1}^{0}=0=x_{2}^{0}$, usando a notação $\Pi_{I I_{z}}$ para os campos da eq. (4.2)

$$
\mathcal{T}_{0}^{\Pi}\left(x_{1}, x_{2}\right)=\Pi_{1}\left(x_{1}^{0}+1 / 2, \vec{x}_{1}\right) \Pi_{1}\left(x_{2}^{0}+1 / 2, \vec{x}_{2}\right)=\Pi_{00}\left(x_{1}^{0}+1 / 2, \vec{x}_{1}\right) \Pi_{00}\left(x_{2}^{0}+1 / 2, \vec{x}_{2}\right) .
$$

Para o acoplamento de dois isovetores com isospin total um tomamos a combinação de coeficientes de $\mathrm{C}-\mathrm{G}$, $\operatorname{com} x_{1}^{0}=0=x_{2}^{0}$,

$$
\begin{aligned}
\mathcal{T}_{1}^{\Pi}\left(x_{1}, x_{2}\right)= & -\frac{1}{\sqrt{3}}\left[\Pi_{2}\left(x_{1}^{0}+1 / 2, \vec{x}_{1}\right) \Pi_{3}\left(x_{2}^{0}+1 / 2, \vec{x}_{2}\right)+\Pi_{4}\left(x_{1}^{0}+1 / 2, \vec{x}_{1}\right) \Pi_{4}\left(x_{2}^{0}+1 / 2, \vec{x}_{2}\right)\right. \\
& \left.+\Pi_{3}\left(x_{1}^{0}+1 / 2, \vec{x}_{1}\right) \Pi_{2}\left(x_{2}^{0}+1 / 2, \vec{x}_{2}\right)\right] \\
= & \frac{1}{\sqrt{3}}\left[\Pi_{11}\left(x_{1}^{0}+1 / 2, \vec{x}_{1}\right) \Pi_{1-1}\left(x_{2}^{0}+1 / 2, \vec{x}_{2}\right)-\Pi_{10}\left(x_{1}^{0}+1 / 2, \vec{x}_{1}\right) \Pi_{10}\left(x_{2}^{0}+1 / 2, \vec{x}_{2}\right)\right. \\
& \left.+\Pi_{1-1}\left(x_{1}^{0}+1 / 2, \vec{x}_{1}\right) \Pi_{11}\left(x_{2}^{0}+1 / 2, \vec{x}_{2}\right)\right] .
\end{aligned}
$$

Como não sabemos a priori qual a combinação linear destes estados é mais apropriada para descrever um possível estado ligado, consideramos a matriz de produtos internos, com $x_{1}^{0}=$ 
$0=x_{2}^{0}=x_{3}^{0}=x_{4}^{0}$ e $i, j=0,1$,

$$
\left(\mathcal{T}_{i}^{\Pi}\left(x_{1}, x_{2}\right), e^{-H\left|x^{0}\right|} e^{i \vec{P} \cdot \vec{x}} \mathcal{T}_{j}^{\Pi}\left(x_{3}, x_{4}\right)\right)_{\mathcal{H}}
$$

Nós notamos que isto é análogo a teoria de perturbação degenerada em mecânica quântica ordinária, e a combinação linear certa deve aparecer no final de nossa análise.

A função de correlação para dois mésons é,

$$
M\left(x_{1}, x_{2}, x_{3}, x_{4}\right)=\left(\begin{array}{cc}
M_{00}\left(x_{1}, x_{2}, x_{3}, x_{4}\right) & M_{01}\left(x_{1}, x_{2}, x_{3}, x_{4}\right) \\
M_{10}\left(x_{1}, x_{2}, x_{3}, x_{4}\right) & M_{11}\left(x_{1}, x_{2}, x_{3}, x_{4}\right)
\end{array}\right)
$$

$\operatorname{com} i, j=0,1$,

$$
M_{i j}=\left\langle\mathcal{T}_{i}^{\mu}\left(x_{1}, x_{2}\right) ; \mathcal{T}_{j}^{\Pi}\left(x_{3}, x_{4}\right)\right\rangle_{T} \chi_{x_{2}^{0} \leq x_{3}^{0}}+\left\langle\mathcal{T}_{i}^{\Pi}\left(x_{1}, x_{2}\right) ; \mathcal{T}_{j}^{\mu}\left(x_{3}, x_{4}\right)\right\rangle_{T}^{*} \chi_{x_{2}^{0}>x_{3}^{0}},
$$

onde $\mathcal{T}_{i}^{\mu}$ é definido similarmente à $\mathcal{T}_{i}^{\Pi}$ com $\mu$ no lugar de $\Pi$.

Definindo, para $x_{2}^{0} \leq x_{3}^{0}$,

$$
D_{l_{1} l_{2} l_{3} l_{4}}\left(x_{1}, x_{2}, x_{3}, x_{4}\right) \equiv\left\langle\mu_{l_{1}}\left(x_{1}\right) \mu_{l_{2}}\left(x_{2}\right) \Pi_{l_{3}}\left(x_{3}\right) \Pi_{l_{4}}\left(x_{4}\right)\right\rangle-\left\langle\mu_{l_{1}}\left(x_{1}\right) \mu_{l_{2}}\left(x_{2}\right)\right\rangle\left\langle\Pi_{l_{3}}\left(x_{3}\right) \Pi_{l_{4}}\left(x_{4}\right)\right\rangle
$$

vemos que, em termos de médias dos campos $\mu_{l}$ e $\Pi_{l}, M_{i j}$ é dado por, para $x_{2}^{0} \leq x_{3}^{0}$ (e de forma similar definimos $x_{2}^{0}>x_{3}^{0}$ usando a Proposição 1 , e suprimindo os pontos da rede para simplificar a notação,

$$
\begin{aligned}
& M_{00}=D_{1111}, \\
& M_{01}=-\frac{1}{\sqrt{3}}\left[D_{1123}+D_{1144}+D_{1132}\right] \\
& M_{10}=-\frac{1}{\sqrt{3}}\left[D_{2311}+D_{4411}+D_{3211}\right], \\
& M_{11}=\frac{1}{3}\left[D_{2323}+D_{2344}+D_{2332}+D_{4432}+D_{4444}+D_{4423}+D_{3223}+D_{3244}+D_{3232}\right] .
\end{aligned}
$$

$M_{0}$ é dado por

$$
M_{0}\left(x_{1}, x_{2}, x_{3}, x_{4}\right)=\left(\begin{array}{ll}
M_{0,00}\left(x_{1}, x_{2}, x_{3}, x_{4}\right) & M_{0,01}\left(x_{1}, x_{2}, x_{3}, x_{4}\right) \\
M_{0,10}\left(x_{1}, x_{2}, x_{3}, x_{4}\right) & M_{0,11}\left(x_{1}, x_{2}, x_{3}, x_{4}\right)
\end{array}\right)
$$


com as entradas

$$
\begin{aligned}
M_{0,00}= & D_{0,1111}, \\
M_{0,01}= & -\frac{1}{\sqrt{3}}\left[D_{0,1123}+D_{0,1144}+D_{0,1132}\right] \\
M_{0,10}= & -\frac{1}{\sqrt{3}}\left[D_{0,2311}+D_{0,4411}+D_{0,3211}\right] \\
M_{0,11}= & \frac{1}{3}\left[D_{0,2323}+D_{0,2344}+D_{0,2332}+D_{0,4432}\right. \\
& \left.+D_{0,4444}+D_{0,4423}+D_{0,3223}+D_{0,3244}+D_{0,3232}\right] .
\end{aligned}
$$

onde $D_{0, l_{1} l_{2} l_{3} l_{4}}$ é obtido aplicando errôneamente o Teorema de Wick aos campos compostos $\mu_{l}$ e $\Pi_{l}$ em $D_{l_{1} l_{2} l_{3} l_{4}}$ sem o truncamento parcial. Explicitamente temos

$$
D_{0, l_{1} l_{2} l_{3} l_{4}}\left(x_{1}, x_{2}, x_{3}, x_{4}\right) \equiv G_{l_{1} l_{3}}\left(x_{1}, x_{3}\right) G_{l_{2} l_{4}}\left(x_{2}, x_{4}\right)+G_{l_{1} l_{4}}\left(x_{1}, x_{4}\right) G_{l_{2} l_{3}}\left(x_{2}, x_{3}\right)
$$

com $G$ definido na eq. (4.6). Nós observamos que

$$
M_{0,01}=M_{0,10}=0
$$

e

$$
M_{0,11}=\frac{1}{3}\left[D_{0,2323}+D_{0,2332}+D_{0,4444}+D_{0,3223}+D_{0,3232}\right],
$$

lembrando que, devido à simetria de isospin, temos $\left\langle\mu_{k}(x) \Pi_{l}(y)\right\rangle=0$ se $k \neq l$. Usando a simetria de conjugação de carga $\mathcal{C}$, temos $\left\langle\mu_{2}(x) \Pi_{2}(y)\right\rangle=\left\langle\mu_{3}(x) \Pi_{3}(y)\right\rangle$. Usando uma rotação de $\pi / 4$, em relação ao eixo $x$-isospin, vemos que $\left\langle\mu_{2}(x) \Pi_{2}(y)\right\rangle=\left\langle\mu_{4}(x) \Pi_{4}(y)\right\rangle$. Desta forma, obtemos uma expressão compacta para os elementos da matriz $2 \times 2 M_{0}$

$$
M_{0,00}=D_{0,1111}, \quad M_{0,11}=D_{0,2222} \text { e } M_{0,01}=M_{0,10}=0 .
$$

Desta forma, a estrutura matricial para $M_{0}$ pode ser simplificada para

$$
M_{0}\left(x_{1}, x_{2}, x_{3}, x_{4}\right)=\left(\begin{array}{cc}
D_{0,1111}\left(x_{1}, x_{2}, x_{3}, x_{4}\right) & 0 \\
0 & D_{0,2222}\left(x_{1}, x_{2}, x_{3}, x_{4}\right)
\end{array}\right)
$$

De forma similar ao que fizemos na demonstração do Teorema 5, podemos mostrar que existem duas partículas distintas, correspondendo ao isospin total $I=1,0$, manifestadas pelo aparecimento de curvas de dispersão isoladas no espectro de energia-momento. O isovetor ou tripleto está associado com $\Pi_{l=2,3,4}(I=1)$ e o isoescalar ou singleto com $\Pi_{1}(I=0)$. Lembrando que $\left\langle\mu_{2}(x) \Pi_{2}(y)\right\rangle=\left\langle\mu_{3}(x) \Pi_{3}(y)\right\rangle=\left\langle\mu_{4}(x) \Pi_{4}(y)\right\rangle$, as curvas de dispersão para as componentes do tripleto $\Pi_{l}=2,3,4$ coincidem. Pela simetria de conjugação de carga, 
$\mathcal{C} \Pi_{1} \mapsto-\Pi_{1}, \mathcal{C} \Pi_{2} \mapsto-\Pi_{3}, \mathcal{C} \Pi_{3} \mapsto-\Pi_{2}$ e $\mathcal{C} \Pi_{4} \mapsto-\Pi_{4}$, e vemos que as curvas de dispersão para cada uma destas partículas coincidem com as das suas antipartículas. As curvas de disersão para o singleto e as componentes do tripleto coincidem até, e incluindo, a ordem $\kappa^{3}$ e são dadas por

$$
w_{I}(\vec{p})=-2 \ln \kappa+r_{I}(\kappa, \vec{p})=-2 \ln \kappa+\ln \left[1-\frac{\kappa^{2}}{2}\left(\cos p^{1}+\cos p^{2}\right)\right]+\mathcal{O}\left(\kappa^{4}\right),
$$

com, $I=0,1, r_{I}(\kappa, \vec{p})$ real e analítica em $\kappa$ e cada componente $p^{j}(j=1,2)$. Claramente, $w_{I}(\vec{p}) \approx m_{I}+\frac{\kappa^{2}}{4}|\vec{p}|^{2},|\vec{p}| \ll 1$, onde $m_{I} \equiv m_{I}(\kappa)=w_{I}(\overrightarrow{0})$ é a massa do méson $I=1$, 0 . As duas massas $m_{0}$ e $m_{1}$ são iguais até e incluindo a ordem $\kappa^{3}$ mas esperamos que uma abertura de massa apareça em ordens superiores de $\kappa$, já que não há nenhuma simetria aparente relacionando as partículas associadas. Na demonstração do Teorema 5 do Capítulo 2, mostramos como obter a abertura de massa em $(2+1)$ e $(3+1)$ dimensões, um sabor e usando matrizes de Dirac $4 \times 4$. É conveniente, por simplicidade de notação, escrever $m_{I}=m^{\prime}+\mathcal{O}\left(\kappa^{4}\right), I=0,1$,

$$
m^{\prime}=-2 \ln \kappa-\kappa^{2}
$$

\subsection{Simetria de isospin $\mathrm{U}(2)$ global e funções de correlação}

Nesta seção usamos a simetria de isospin para obter as relações de ortogonalidade entre funções de correlação de dois pontos, i.e. $\left\langle\mu_{k}(x) \Pi_{l}(y)\right\rangle=0$, para $k \neq l, k, l=1,2,3,4$. Estas relações são análogas às usuais relações de ortogonalidade para estados com diferentes componentes do momento angular na direção $z$ e diferentes momentos angulares totais na mecânica quântica ordinária. As funções de correlação $\mu$-П são combinações lineares de $\left\langle\psi_{f_{1}} \bar{\psi}_{f_{2}} \bar{\psi}_{f_{3}} \psi_{f_{4}}\right\rangle$, onde suprimimos todos índices exceto aqueles referentes ao isospin ou sabor. Começamos com a identidade, para $U \in \mathrm{U}(2)$ arbitrário, dada por

$$
\left\langle\psi_{f_{1}} \bar{\psi}_{f_{2}}(\bar{\psi} U)_{f_{3}}\left(U^{\dagger} \psi\right)_{f_{4}}\right\rangle=\left\langle(U \psi)_{f_{1}}\left(\bar{\psi} U^{\dagger}\right)_{f_{2}} \bar{\psi}_{f_{3}} \psi_{f_{4}}\right\rangle
$$

onde o lado direito da equação anterior é obtido a partir do lado esquerdo usando simetria de isospin, i.e., invariância das médias pela transformação $\psi \mapsto U \psi$ e $\bar{\psi} \mapsto \bar{\psi} U^{\dagger} \operatorname{com} U \in U(2)$. Multiplicando ambos os lados pela esquerda pelo tensor $w_{f_{1} f_{2}}$ e $v_{f_{3} f_{4}}$ pela direita, obtemos nossa identidade básica

$$
w_{f_{1} f_{2}}\left\langle\psi_{f_{1}} \bar{\psi}_{f_{2}} \bar{\psi}_{i_{3}} \psi_{i_{4}}\right\rangle U_{i_{3} f_{3}} \bar{U}_{i_{4} f_{4}} v_{f_{3} f_{4}}=w_{i_{1} i_{2}} U_{i_{1} f_{1}} \bar{U}_{i_{2} f_{2}}\left\langle\psi_{f_{1}} \bar{\psi}_{f_{2}} \bar{\psi}_{i_{3}} \psi_{i_{4}}\right\rangle v_{f_{3} f_{4}}
$$

onde a barra em $U$ significa conjugação complexa. Denotando $\langle\cdot\rangle_{f_{1} f_{2} ; f_{3} f_{4}}$ por $T_{f_{1} f_{2} ; f_{3} f_{4}}$, podemos reescrever a expressão acima como $w_{12} T_{12 ; 34}[(U \otimes \bar{U}) v]_{34}=[w(U \otimes \bar{U})]_{12} T_{12 ; 34} v_{34}$. Então, vemos 
que o produto tensorial de $U$ e $\bar{U}$ ocorre em fatores distintos. Relembrando que para $U \in \mathrm{SU}(2)$ arbitrário, temos (veja ref. [24]), onde $\sigma_{x} \equiv \sigma^{1}, \sigma_{y} \equiv \sigma^{2}$ e $\sigma_{z} \equiv \sigma^{3}$ são as matrizes de Pauli da eq. (1.4) e

$$
\bar{U}=S U S^{-1}, S=i \sigma_{y}=-S^{T}=\bar{S}=-S^{-1},
$$

de forma que

$$
U \otimes \bar{U}=U \otimes\left(S U S^{-1}\right)=(1 \otimes S)(U \otimes U)\left(1 \otimes S^{-1}\right) .
$$

Escrevendo $U=e^{i \theta \sigma / 2}$, onde $\theta \sigma=\theta_{x} \sigma_{x}, \theta_{y} \sigma_{y}, \theta_{z} \sigma_{z}, \theta_{x, y, z} \in \mathbb{R}$, expandindo em potências de $\theta$ e igualando o termo linear para $\theta \sigma=\theta_{z} \sigma_{z}$, e a soma dos termos $\theta_{x}^{2}$, $\theta_{y}^{2}$ e $\theta_{z}^{2}$, obtemos, com $\vec{I}=\vec{\sigma} / 2=\left(I_{x}, I_{y}, I_{z}\right)$

$$
w_{12} T_{12 ; 34}\left[\left(I_{z}^{\prime}\right) v\right]_{34}=\left[w\left(I_{z}^{\prime}\right)\right]_{12} T_{12 ; 34} v_{34}
$$

onde $I_{z}^{\prime}=I_{1 z} \otimes 1+1 \otimes\left(S I_{2 z} S^{-1}\right)$.

Para o coeficiente da soma dos termos quadráticos, nós temos

$$
w_{12} T_{12 ; 34}\left[\left(I^{\prime}\right)^{2} v\right]_{34}=\left[w\left(I^{\prime}\right)^{2}\right]_{12} T_{12 ; 34} v_{34},
$$

onde $\left(I^{\prime}\right)_{i j}^{2}=\sum_{k=x, y, z}\left[I_{i k} \otimes 1+1 \otimes\left(S I_{j k} S^{-1}\right)\right]^{2}$. Ressaltamos que se o produto de representações tensoriais $U \otimes U$ ocoresse, ao invés de $U \otimes \bar{U}$, então os operadores de isospin acima seriam os usuais $I_{z}$, a componente $z$ do isospin total, e $I^{2}$, o isospin total ao quadrado. Com a notação usual de momento angular, com um produto tensorial sub-entendido, denotamos as auto-funções usuais do isospin total e a componente $z$ do isospin total pelo tripleto

$$
\chi_{10}=\frac{1}{\sqrt{2}}(\alpha \beta+\beta \alpha), \chi_{11}=\alpha \alpha, \chi_{1-1}=\beta \beta,
$$

e o singleto $\chi_{00}=\frac{1}{\sqrt{2}}(\alpha \beta-\beta \alpha)$, onde

$$
\alpha=\left(\begin{array}{l}
1 \\
0
\end{array}\right) \quad \text { e } \beta=\left(\begin{array}{l}
0 \\
1
\end{array}\right)
$$

Obviamente, o auto-valor de $I^{2}$ é $I(I+1)=2$ (respectivamente, 0 ) e os auto-valores de $I_{z}$ são $0,1,-1$ (respectivamente, 0 ).

As auto-funções de $\left(I^{\prime}\right)^{2}$ são dadas por

$$
\chi_{\text {Im }}^{\prime}=(1 \otimes S) \chi_{\text {Im }}
$$

ou seja

$$
\chi_{10}^{\prime}=\frac{1}{\sqrt{2}}(\alpha \alpha-\beta \beta), \chi_{11}^{\prime}=\alpha(-\beta), \chi_{1-1}^{\prime}=\beta \alpha,
$$


e $\chi_{00}^{\prime}=\frac{1}{\sqrt{2}}(\alpha \alpha+\beta \beta)$, e elas têm os mesmos auto-valores das correspondentes $\chi_{\text {Im }}$ 's. Nós notamos que por uma inspeção dos índices de isospin na eq. (4.2) fazemos a identificação $\chi_{10}^{\prime} \mapsto \Pi_{4}, \chi_{11}^{\prime} \mapsto-\Pi_{2}, \chi_{1-1}^{\prime} \mapsto \Pi_{3}$ e $\chi_{00}^{\prime} \mapsto \Pi_{1}$.

Usando estas auto-funções com ' nas igualdades (4.12) e (4.13) obtemos a forma da função de correlação $\left\langle\mu_{k} \Pi_{l}\right\rangle$ como combinações lineares de $\left\langle\psi_{f_{1}} \bar{\psi}_{f_{2}} \bar{\psi}_{f_{3}} \psi_{f_{4}}\right\rangle$ e também as relações de ortogonalidade. Por exemplo, para $v=\chi_{10}^{\prime}$, tomamos $v_{f_{3} f_{4}}=\frac{1}{\sqrt{2}}\left[\delta_{f_{3}+} \delta_{f_{4}+}-\delta_{f_{3}-} \delta_{f_{4}-}\right]$, etc. Aqui, usamos o fato que o operador $\vec{\sigma} \cdot\left(S \vec{\sigma} S^{-1}\right)$ tem a mesma ação à esquerda ou à direita de $w_{i_{1} i_{2}}$.

Podemos estender nossa análise de isospin para estados de duas partículas considerando a função de quatro pontos $\left\langle\mu_{l_{1}} \mu_{l_{2}} \Pi_{l_{3}} \Pi_{l_{4}}\right\rangle$. Nós temos a identidade, para $U \in \mathrm{U}(2)$,

$$
\left\langle\psi_{f_{1}} \bar{\psi}_{f_{2}} \psi_{f_{3}} \bar{\psi}_{f_{4}}(\bar{\psi} U)_{f_{5}}\left(U^{\dagger} \psi\right)_{f_{6}}(\bar{\psi} U)_{f_{7}}\left(U^{\dagger} \psi\right)_{f_{8}}\right\rangle=\left\langle(U \psi)_{f_{1}}\left(\bar{\psi} U^{\dagger}\right)_{f_{2}}(U \psi)_{f_{3}}\left(\bar{\psi} U^{\dagger}\right)_{f_{4}} \bar{\psi}_{f_{5}} \psi_{f_{6}} \bar{\psi}_{f_{7}} \psi_{f_{8}}\right\rangle
$$

ou, com notação similar a acima,

$$
T_{1234 ; 5678}(U \otimes \bar{U} \otimes U \otimes \bar{U})=(U \otimes \bar{U} \otimes U \otimes \bar{U}) T_{1234 ; 5678}
$$

O produto tensorial de quatro campos $U \otimes \bar{U} \otimes U \otimes \bar{U}$ pode ser decomposto como $(U \otimes \bar{U}) \otimes$ $(U \otimes \bar{U})$ e, conforme vimos, cada fator $U \otimes \bar{U}$ tem auto-funções de isospin $\chi_{10}^{\prime}, \chi_{11}^{\prime}, \chi_{1-1}^{\prime}$ e $\chi_{00}^{\prime}$. Para o produto tensorial, temos uma base de dezesseis dimensões que pode ser decomposta em isospin total dois, um (multiplicidade três) e zero (multiplicidade dois).

Nós agora estamos prontos para calcular a aproximação $L$ para $K$ e resolver a equação de B-S nesta aproximação.

\subsection{A aproximação escada para a equação de Bethe-Salpeter: caso méson-méson, dois sabores}

Nós aqui passamos direto à consideração da aproximação escada para $K$. Considerações a respeito da existência de $M, M_{0}, M^{-1}, M_{0}^{-1}$ seguem como no capítulo anterior. Mencionamos apenas que, para incorporar o grau de liberdade interno isospin, devemos considerar a ação destes operadores no espaço $\ell_{2}^{s}(\mathrm{~A})$ subespaço simétrico, em relação às coordenadas e ao isospin, de $\ell_{2}(\mathrm{~A})$ onde $\mathrm{A}=\left\{\left(x_{1} i_{1} ; x_{2} i_{2}\right) \in\left(\mathbb{Z}^{d} \times\{0,1\}\right) \times\left(\mathbb{Z}^{d} \times\{0,1\}\right) ; x_{1}^{0}=x_{2}^{0}\right\}$. Como no capítulo anterior usamos o superscrito $(n)=(0),(1), \ldots$ para denotar o coeficiente de $\kappa^{n}$ e o argumento 
0 para denotar sítios coincidentes $x_{1}=x_{2}=x_{3}=x_{4}$, a expansão para $K$ é obtida escrevendo

$$
\begin{aligned}
K= & {\left[M_{0}^{(0)}+\delta M_{0}\right]^{-1}-\left[M^{(0)}+\delta M\right]^{-1} } \\
= & \left(M_{0}^{(0)}\right)^{-1}-\left(M^{(0)}\right)^{-1}+\sum_{n=1}^{\infty}(-1)^{n}\left\{\left[\left(M_{0}^{(0)}\right)^{-1}\right.\right. \\
& \left.\left.\times \delta M_{0}\right]^{n}\left(M_{0}^{(0)}\right)^{-1}-\left[\left(M^{(0)}\right)^{-1} \delta M\right]^{n}\left(M^{(0)}\right)^{-1}\right\},
\end{aligned}
$$

onde $\delta M_{0} \equiv M_{0}-M_{0}^{(0)}$ e $\delta M \equiv M-M^{(0)}$ admitem expansões em $\kappa$. Para obter a inversa de $M^{(0)}$ procedemos como no capítulo anterior, na Seção 3.3, escrevendo o núcleo de $M^{(0)}$ como

$$
\begin{aligned}
M^{(0)}\left(x_{1}, x_{2}, x_{3}, x_{4}\right)= & M^{(0)}(0) \delta\left(x_{1}-x_{3}\right) \delta\left(x_{2}-x_{4}\right) \\
& \times \delta\left(x_{1}-x_{2}\right)+M_{0}^{(0)}\left(x_{1}, x_{2}, x_{3}, x_{4}\right) \\
& \times\left[1-\delta\left(x_{1}-x_{2}\right) \delta\left(x_{1}-x_{3}\right) \delta\left(x_{1}-x_{4}\right)\right],
\end{aligned}
$$

que coincide com $M_{0}^{(0)}$ exceto em pontos coincidentes. Para determinarmos a matriz $2 \times 2$ $M^{(0)}(0)$ precisamos, somente, das seguintes entradas (usando o Teorema de Wick):

$$
D_{1111}^{(0)}(0)=D_{4444}^{(0)}(0)=\frac{5}{3}, D_{1123}^{(0)}(0)=D_{1144}^{(0)}(0)=-\frac{1}{3}, D_{2323}^{(0)}(0)=1 \text { e } D_{2344}^{(0)}(0)=\frac{1}{3} .
$$

As outras entradas de $M^{(0)}(0)$ são obtidas usando as propriedades de simetria de $D$, i.e.

$$
D_{l_{1} l_{2} l_{3} l_{4}}\left(x_{1}, x_{2}, x_{3}, x_{4}\right)=D_{l_{2} l_{1} l_{3} l_{4}}\left(x_{2}, x_{1}, x_{3}, x_{4}\right)=D_{l_{1} l_{2} l_{4} l_{3}}\left(x_{1}, x_{2}, x_{4}, x_{3}\right)
$$

e a reversão no tempo do Teorema 1 , que fornece $D_{l_{1} l_{2} l_{3} l_{4}}^{(0)}(0)=D_{l_{3} l_{4} l_{1} l_{2}}^{(0)}(0)$. Desta forma,

$$
M^{(0)}(0)=\frac{1}{3}\left(\begin{array}{cc}
5 & \sqrt{3} \\
\sqrt{3} & 7
\end{array}\right)=\frac{8}{3} P+\frac{4}{3} P_{1},
$$

onde na última igualdade da eq. (4.15) usamos a decomposição espectral de $M^{(0)}(0)$. $P, P_{1}$ são projeções ortogonais associadas aos autovalores $8 / 3$ e $4 / 3$ de $M^{(0)}(0)$, com autovetores normalizados $v=\frac{1}{2}(1, \sqrt{3})$ e $v_{1}=\frac{1}{2}(\sqrt{3},-1)$, ou seja

$$
P=\frac{1}{4}\left(\begin{array}{cc}
1 & \sqrt{3} \\
\sqrt{3} & 3
\end{array}\right)
$$

e

$$
P_{1}=\frac{1}{4}\left(\begin{array}{cc}
3 & -\sqrt{3} \\
-\sqrt{3} & 1
\end{array}\right)
$$


Relembrando que $G_{k l}$ é diagonal e normalizado em $\kappa=0$ e em pontos coincidentes, obtemos

$$
M_{0, i j}^{(0)}\left(x_{1}, x_{2}, x_{3}, x_{4}\right)=\delta_{i j}\left[\delta\left(x_{1}-x_{3}\right) \delta\left(x_{2}-x_{4}\right)+\delta\left(x_{1}-x_{4}\right) \delta\left(x_{2}-x_{3}\right)\right]
$$

e $M_{0}^{(0)}$ age como duas vezes a identidade em $\ell_{2}^{s}(\mathrm{~A})$. Desta forma concluímos que

$$
\left(M_{0}^{(0)}\right)^{-1}=\frac{1}{2} I_{2} \delta\left(x_{1}-x_{3}\right) \delta\left(x_{2}-x_{4}\right)
$$

Para listarmos todas as contribuições que entram no cálculo de $L$, consideramos o decaimento no núcleo de $K$ dado pela eq. (B.37) do Apêndice B. Nós obtemos que $K$ é $\mathcal{O}\left(\kappa^{2}\right)$ e listamos abaixo as possibilidades que devem ser consideradas para distâncias temporais $x_{3}^{0}-x_{1}^{0}=0, e^{0}, 2 e^{0}$. Note que para $\left|x_{3}^{0}-x_{2}^{0}\right| \geq 3$ temos uma contribuição de pelo menos $\mathcal{O}\left(\kappa^{4}\right)$ que não contribui para $L$. Para determinamos as contribuições que devem ser consideradas lembramos que estamos buscando as singularidades de $\hat{M}\left(\vec{\xi}, \vec{\eta}, k^{0}\right)$ com $k^{0}=i \chi=i\left(2 m^{\prime}-\bar{\epsilon}\right)$ dependente da energia e contribuindo com um fator $e^{-i k_{0}}=e^{\chi}=e^{2 m^{\prime}-\bar{\epsilon}}=e^{-\left(\bar{\epsilon}+2 \kappa^{2}\right)} / \kappa^{4}$, onde $m^{\prime}$ é dado pela eq. (4.11). Desta forma obtemos:

(I) $\left|x_{3}^{0}-x_{1}^{0}\right|=0,\left|\vec{x}_{1}+\vec{x}_{2}-\vec{x}_{3}-\vec{x}_{4}\right|_{1}+\left|\vec{x}_{2}-\vec{x}_{1}\right|_{1}+\left|\vec{x}_{4}-\vec{x}_{3}\right|_{1}=0 \Rightarrow|K|=\mathcal{O}(1)$ e $e^{\left(2 m^{\prime}-\bar{\epsilon}\right)\left|\tau^{0}\right|}=1$, contribuindo para $\hat{K}(\vec{\xi}, \vec{\eta}) \operatorname{com}\left(P / 8-P_{1} / 4\right) \delta(\vec{\xi}) \delta(\vec{\eta}) \delta(\tau)$, que corresponde a um potencial de alcance zero com uma parte atrativa $(P / 8)$ e outra repulsiva $\left(-P_{1} / 4\right)$;

Diagramaticamente temos a contribuição trivial

$$
x_{1}=x_{2}=x_{3}=x_{4}
$$

Para o termo de ordem zero, i.e. $K^{(0)}$, temos

$$
K^{(0)}\left(x_{1}, x_{2}, x_{3}, x_{4}\right)=\left[\left(M_{0}^{(0)}(0)\right)^{-1}-\left(M^{(0)}(0)\right)^{-1}\right] \delta\left(x_{1}-x_{3}\right) \delta\left(x_{2}-x_{4}\right) \delta\left(x_{1}-x_{2}\right) .
$$

Para determinarmos o valor de $K^{(0)}$ usamos a eq. (4.15) para obtermos $\left[M^{(0)}(0)\right]^{-1}=$ $\frac{3}{8} P+\frac{3}{4} P_{1}$ e relembrando que $M_{0}^{(0)}(0)=2 I_{2}=2\left(P+P_{1}\right)$ e $\left[M_{0}^{(0)}(0)\right]^{-1}=\frac{1}{2}\left(P+P_{1}\right)$, temos

$$
\begin{aligned}
\left(M_{0}^{(0)}\right)^{-1}-\left(M^{(0)}\right)^{-1} & =K^{(0)}\left(x_{1}, x_{2}, x_{3}, x_{4}\right) \\
& =K^{(0)}(0) \delta\left(x_{1}-x_{3}\right) \delta\left(x_{2}-x_{4}\right) \delta\left(x_{1}-x_{2}\right) \\
& =\left(\frac{P}{8}-\frac{P_{1}}{4}\right) \delta\left(x_{1}-x_{3}\right) \delta\left(x_{2}-x_{4}\right) \delta\left(x_{1}-x_{2}\right)
\end{aligned}
$$

Observação 7 Conforme veremos, (I) irá combinar com (III) para obtermos efetivamente uma contribuição da ordem $\kappa^{2}$. 
(II) $\left|x_{3}^{0}-x_{1}^{0}\right|=0,\left|\vec{x}_{1}+\vec{x}_{2}-\vec{x}_{3}-\vec{x}_{4}\right|_{1}+\left|\vec{x}_{2}-\vec{x}_{1}\right|_{1}+\left|\vec{x}_{4}-\vec{x}_{3}\right|_{1}=2 \Rightarrow|K|=\mathcal{O}\left(\kappa^{2}\right)$ e $e^{\left(2 m^{\prime}-\bar{\epsilon}\right)\left|\tau^{0}\right|}=1$, contribuindo para $\hat{K}(\vec{\xi}, \vec{\eta}) \operatorname{com} \kappa^{2}\left(P_{-} / 6\right) \delta(\vec{\xi}-\vec{\eta}) \delta\left(\vec{\xi}-\epsilon e^{i}\right) \delta\left(\tau+\epsilon e^{i}\right)$. Este potencial é de alcance um no espaço e está associado com a troca de um quasi-méson;

Diagramaticamente temos

$$
x _ { 1 } = x _ { 3 } = 0 \longdiv { < } \epsilon e ^ { i } = x _ { 2 } = x _ { 4 }
$$

$\mathrm{Ou}$

$$
x_{2}=x_{4}=0 \longrightarrow
$$

Esta contribuição é obtida da mesma que forma que fizemos para calcular a aproximação escada para $K$ no capítulo anterior, mais especificamente na Seção 3.3. Notamos, no entanto, que, como não estamos usando o fator $h$ neste caso, as contribuições não-locais, dadas por (vii) e (viii), são zero. Neste caso, a única contribuição diferente de zero é (v). Para este caso, expandindo $\left\langle\mu_{l_{1}}(0) \mu_{l_{2}}\left(e^{1}\right) \Pi_{l_{3}}(0) \Pi_{l_{4}}\left(e^{1}\right)\right\rangle$ em potências de $\kappa$ e considerando, por exemplo, $\delta M_{00}^{(2)}$, a entrada da matriz $\delta M^{(2)}$ com $k=0=l$ (associada ao setor de isospin total zero), temos

$$
\delta M_{00}^{(2)}=\frac{1}{12}\left\langle\mu_{1} \Pi_{1} \bar{\psi}_{\alpha_{1}, a, f_{1}} \psi_{\beta_{2}, a, f_{2}}(0)\right\rangle^{(0)}\left\langle\psi_{\beta_{1}, b, f_{1}} \bar{\psi}_{\alpha_{2}, b, f_{2}} \mu_{1} \Pi_{1}\left(e^{1}\right)\right\rangle^{(0)} \Gamma_{\alpha_{1} \beta_{1}}^{e^{1}} \Gamma_{\alpha_{2} \beta_{2}}^{-e^{1}}
$$

Agora, usando a estrutura explícita das matrizes $\Gamma$, segue que

$$
\begin{aligned}
\delta M_{00}^{(2)}= & \frac{1}{12}\left\langle\mu_{1} \Pi_{1}\left(\bar{\psi}_{+, a, f_{1}} \psi_{+, a, f_{2}}-\bar{\psi}_{-, a, f_{1}} \psi_{-, a, f_{2}}\right)(0)\right\rangle^{(0)} \\
& \times\left\langle\left(\psi_{+, b, f_{1}} \bar{\psi}_{+, b, f_{2}}-\psi_{-, b, f_{1}} \bar{\psi}_{-, b, f_{2}}\right) \mu_{1} \Pi_{1}\left(e^{1}\right)\right\rangle^{(0)} .
\end{aligned}
$$

Finalmente, usando as definições de $\mu_{1}$ e $\Pi_{1}$ da Seção 4.1, obtemos

$$
\left\langle\mu_{1} \Pi_{1}\left(\bar{\psi}_{+, a, f_{1}} \psi_{+, a, f_{2}}-\bar{\psi}_{-, a, f_{1}} \psi_{-, a, f_{2}}\right)(0)\right\rangle^{(0)}=0
$$

Então, $\delta M_{00}^{(2)}=0$. Nós também obtemos, após um cálculo longo, $\delta M_{01}^{(2)}=0=\delta M_{10}^{(2)} \mathrm{e}$ $\delta M_{11}^{(2)}=\kappa^{2} / 3$. Desta forma $K^{(2)}\left(0, e^{1}, 0, e^{1}\right)=(1 / 4) \delta M^{(2)}\left(0, e^{1}, 0, e^{1}\right)=\left(\kappa^{2} / 12\right) P_{-}$. Levando em conta contribuições do tipo $x_{1}=x_{3}=0, x_{2}=x_{4}=\epsilon e^{j}$ e $x_{1}=x_{4}=0, x_{2}=x_{3}=\epsilon e^{j}$ 
para $\delta M^{(2)}\left(x_{1}, x_{2}, x_{3}, x_{4}\right)$ obtemos o resultado desejado. Este termo está associado com um potencial atrativo, de alcance espacial um e resultante da troca de um quasi-méson. Na nossa terminologia um méson tem índices de spin opostos para os campos de quark e anti-quark, neste caso temos os mesmos índices de spin para o quark e anti-quark.

$\left|x_{3}^{0}-x_{1}^{0}\right|=1,\left|\vec{x}_{1}+\vec{x}_{2}-\vec{x}_{3}-\vec{x}_{4}\right|_{1}+\left|\vec{x}_{2}-\vec{x}_{1}\right|_{1}+\left|\vec{x}_{4}-\vec{x}_{3}\right|_{1}=0 \Rightarrow|K|=\mathcal{O}\left(\kappa^{4}\right)$ e $e^{\left(2 m^{\prime}-\bar{\epsilon}\right)\left|e^{0}\right|}=$ $e^{-\left(\bar{\epsilon}+2 \kappa^{2}\right)} / \kappa^{4}$, contribuindo para $\hat{K}(\vec{\xi}, \vec{\eta}) \operatorname{com} e^{-\left(\bar{\epsilon}+2 \kappa^{2}\right)}\left(P_{1} / 4-P / 8\right) \delta(\vec{\xi}) \delta(\vec{\eta}) \delta\left(\tau-e^{0}\right)$. Esta contribuição corresponde a um potencial dependente da energia de alcance zero. Seu cálculo leva em consideração o efeito da correlação de quatro campos de calibre superpostos, da eq. (A.12);

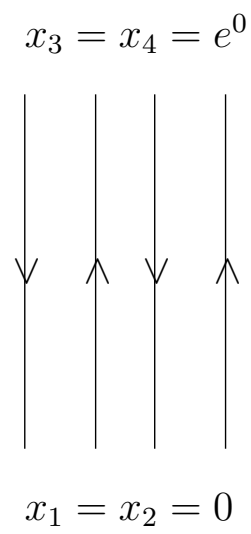

Pela equação (B.30) do Apêndice B temos

$$
\left\{\left[M^{(0)}\right]^{-1} \delta M^{(4)}\left[M^{(0)}\right]^{-1}-\left[M_{0}^{(0)}\right]^{-1} \delta M_{0}^{(4)}\left[M_{0}^{(0)}\right]^{-1}\right\}\left(0,0, e^{0}, e^{0}\right)
$$

e lembrando que $\delta M^{(4)}=(8 / 3) P+(4 / 3) P_{1},\left[M^{(0)}\right]^{-1}=(3 / 8) P+(3 / 4) P_{1}$ e $\delta M_{0}^{(4)}=M_{0}=2 I_{2}$ obtemos o resultado desejado.

(IV) $\left|x_{3}^{0}-x_{1}^{0}\right|=1,\left|\vec{x}_{1}+\vec{x}_{2}-\vec{x}_{3}-\vec{x}_{4}\right|_{1}+\left|\vec{x}_{2}-\vec{x}_{1}\right|_{1}+\left|\vec{x}_{4}-\vec{x}_{3}\right|_{1}=2 \Rightarrow|K|=\mathcal{O}\left(\kappa^{6}\right) \mathrm{e}$ $e^{\left(2 m^{\prime}-\bar{\epsilon}\right)\left|e^{0}\right|}=e^{-\left(\bar{\epsilon}+2 \kappa^{2}\right)} / \kappa^{4} ;$

Uma contribuição típica para este caso tem a representação gráfica 


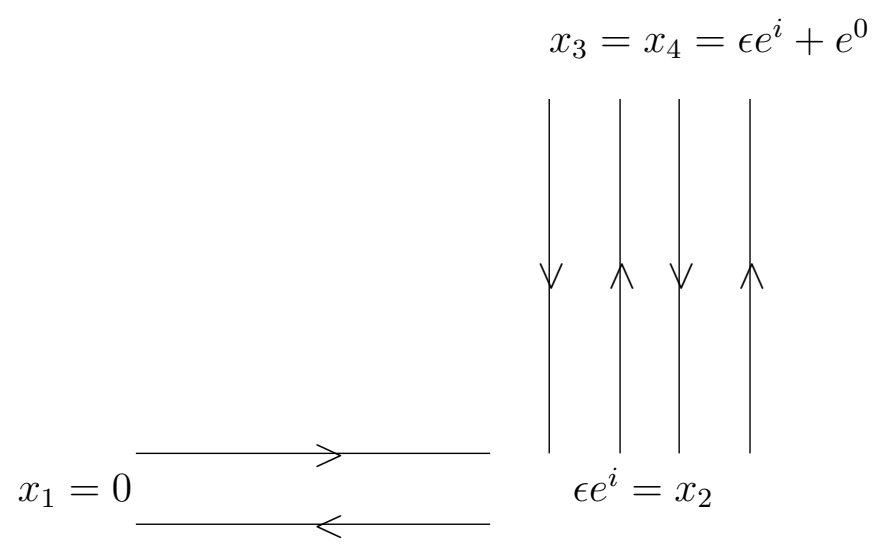

Neste caso, os cálculos são extensos e nos limitaremos a dar apenas a descrição do procedimento utilizado para determinarmos todos os termos envolvidos. Esta contribuição é zero usando a expansão em série de Neumann para $M^{-1}$ e $M_{0}^{-1}$ (veja a eq. (4.14)) e relembrando que $\delta M^{(2)}$ e $\delta M_{0}^{(2)}$ são os coeficientes de termos da série de Neumann para $M^{-1}$ e $M_{0}^{-1}$, respectivamente, pelo menos da ordem $\mathcal{O}\left(\kappa^{2}\right)$. Desta forma, precisamos considerar na expansão de Neumann para $M^{-1}\left(M_{0}^{-1}\right)$ no máximo produtos de três $\delta M^{(2)}\left(\delta M_{0}^{(2)}\right)$. Na sequência, usamos a notação $\vec{u}_{n}=\left(y_{1}, y_{2}, \ldots y_{n}\right)$ para um vetor de $n$ componentes, tal que $y_{i}=2 m_{i}(i=1, \ldots, n), m_{i} \in \mathbb{N}$, submetido ao vínculo $\sum_{i=1}^{n} y_{i}=6$. Assim, (IV) é dada pela soma das contribuições $\delta M^{(6)},-\sum_{\vec{u}_{2}} \delta M^{\left(y_{1}\right)} \delta M^{\left(y_{2}\right)}$ e $\delta M^{(2)} \delta M^{(2)} \delta M^{(2)}$, omitindo os fatores $\left[M^{(0)}\right]^{-1}$ que aparecem na série de Neumann aqui e no que se segue [contribuição (V)] para simplificar a notação, e similarmente para $\left[M_{0}\right]^{-1}$. Pelo decaimento no espaço dado pela eq. (B.37) do Apêndice B obtemos

$$
\left|\vec{x}_{1}+\vec{x}_{2}-\vec{x}_{3}-\vec{x}_{4}\right|_{1} \equiv a, \quad\left|\vec{x}_{2}-\vec{x}_{1}\right|_{1} \equiv b \quad \text { e } \quad\left|\vec{x}_{4}-\vec{x}_{3}\right|_{1} \equiv c
$$

e a condição $a+b+c=2$ deve ser satisfeita. As possibilidades são dadas por (IV.i) $a=2$ e $b=c=0$; (IV.ii) $b=2$ e $a=c=0$; (IV.iii) $c=2$ e $a=b=0$; (IV.iv) $a=0$ e $b=c=1$; (IV.v) $b=0$ e $a=c=1$; (IV.vi) $c=0$ e $a=b=1$. Na sequência, mostramos dois exemplos que mostram um cancelamento não-trivial na expansão usada para calcular $K$. Consideramos, a possibilidade (IV.iv) com, por exemplo, $x_{1}=0, x_{2}=e^{1}, x_{3}=e^{0}$ e $x_{4}=e^{0}+e^{1}$. Neste caso, a contribuição não-nula $\delta M^{(6)}$ é cancelada pelo próximo termo $-\left[\delta M^{(2)} \delta M^{(4)}+\delta M^{(4)} \delta M^{(2)}\right]$ na expansão em série de Neumann e cada termo na expansão para $\left[M_{0}\right]^{-1}$ é zero até esta ordem. A seguir, para (IV.v), se $x_{1}=0=x_{2}, x_{3}=e^{0}$ e $x_{4}=e^{0}+e^{1}$ nós obtemos que $\delta M^{(6)}$ é cancelado pela expressão correspondente vinda de $\left[M_{0}\right]^{-1}$, que é $-\delta M_{0}^{(6)}$ e similarmente para $-\sum_{\vec{u}_{2}} \delta M^{\left(y_{1}\right)} \delta M^{\left(y_{2}\right)}$.

(V) $\left|x_{3}^{0}-x_{1}^{0}\right|=2,\left|\vec{x}_{1}+\vec{x}_{2}-\vec{x}_{3}-\vec{x}_{4}\right|_{1}+\left|\vec{x}_{2}-\vec{x}_{1}\right|_{1}+\left|\vec{x}_{4}-\vec{x}_{3}\right|_{1}=0 \Rightarrow|K|=\mathcal{O}\left(\kappa^{10}\right) \mathrm{e}$ 


$$
e^{\left(2 m^{\prime}-\bar{\epsilon}\right)\left|2 e^{0}\right|}=e^{-2\left(\bar{\epsilon}+2 \kappa^{2}\right)} / \kappa^{8} .
$$

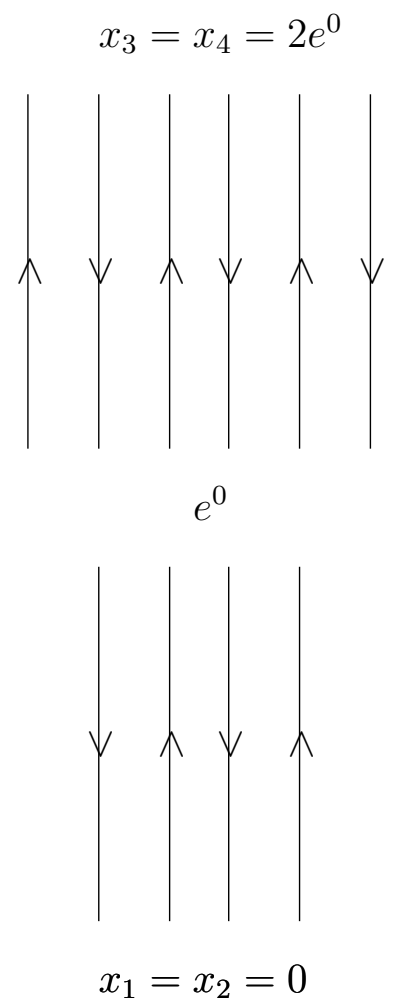

Novamente, como os cálculos envolvidos são extensos nos limitamos a uma discussão breve. Usando a notação da contribuição (IV), i.e. $\vec{u}_{n}=\left(y_{1}, y_{2}, \ldots y_{n}\right)$ indica um vetor de $n$ componentes, tal que $y_{i}=2 m_{i}(i=1, \ldots, n), m_{i} \in \mathbb{N}$, submetido ao vínculo $\sum_{i=1}^{n} y_{i}=10$ e omitimos os fatores $\left[M^{(0)}\right]^{-1}$ e $\left[M_{0}^{(0)}\right]^{-1}$ que aparecem nas séries de Neumann para $\left[M^{(0)}\right]^{-1}$ e $\left[M_{0}^{(0)}\right]^{-1}$. Devemos considerar $\delta M^{(10)},-\sum_{\vec{u}_{2}} \delta M^{\left(y_{1}\right)} \delta M^{\left(y_{2}\right)}, \sum_{\vec{u}_{3}}$ $\delta M^{\left(y_{1}\right)} \delta M^{\left(y_{2}\right)} \delta M^{\left(y_{3}\right)},-\sum_{\vec{u}_{4}} \delta M^{\left(y_{1}\right)} \delta M^{\left(y_{2}\right)} \delta M^{\left(y_{3}\right)} \delta M^{\left(y_{4}\right)}$ e finalmente $\delta M^{(2)} \delta M^{(2)} \delta M^{(2)} \delta M^{(2)} \delta M^{(2)}$. Aqui, cada contribuição é zero e o mesmo acontece para $\left[M_{0}\right]^{-1}$.

Levando em conta os resultados acima (I),.., (V), que contabilizam as contribuições para $L$, obtemos

$$
\begin{aligned}
\hat{L}(\vec{\xi}, \vec{\eta}, i \chi) & =\left(1-e^{-\bar{\epsilon}-2 \kappa^{2}}\right)\left(\frac{1}{8} P-\frac{1}{4} P_{1}\right) \delta(\vec{\xi}) \delta(\vec{\eta})+\frac{1}{3} P_{-} \sum_{\sigma, j} \delta(\vec{\xi}-\vec{\eta}) \delta\left(\vec{\eta}-\sigma e^{j}\right) \\
& =\frac{1}{4}(b+2) \kappa^{2}\left(\frac{1}{8} P-\frac{1}{4} P_{1}\right) \delta(\vec{\xi}) \delta(\vec{\eta})+\frac{2}{3} P_{-} \sum_{j} \delta(\vec{\xi}-\vec{\eta}) \delta\left(\vec{\eta}-e^{j}\right)
\end{aligned}
$$

onde $b \kappa^{2} \equiv 1-e^{-\bar{\epsilon}}$ é a energia de ligação do méson-méson e para obtermos a última igualdade na eq. (4.18) usamos a simetria $\hat{M}\left(\vec{\xi}, \vec{\eta}, k^{0}\right)=\hat{M}\left(-\vec{\xi}, \vec{\eta}, k^{0}\right)=\hat{M}\left(\vec{\xi},-\vec{\eta}, k^{0}\right)$ e o mesmo para $\hat{M}_{0}$ e conseqüentemente para $\hat{K}$.

Observação 8 Notamos que na expressão de $\hat{L}$ da eq. (4.18) supomos $\bar{\epsilon}=\mathcal{O}\left(\kappa^{2}\right)$ o quê, 
conforme veremos é consistente com a energia de ligação obtida através da solução da equação de $B$-S na aproximação escada.

Notamos que, na analogia com operadores de Schrödinger, o potencial dado pela eq. (4.18) corresponde a um potencial local dependente da energia de alcance espacial zero, com uma parte atrativa $\left(\frac{1}{2} P\right)$, e repulsiva $\left(-P_{1}\right)$. Um potencial atrativo de alcance espacial um $\left(\frac{2}{3} P_{-}\right)$correspondendo a troca de um quasi-méson (na nossa terminologia isto significa que, ele não tem os índices de spin adequados para que possamos identificálos com um méson).

De volta à equação de B-S obtemos, deste momento em diante omitimos a coordenada conjugada a $\tau, k=\left(k^{0}, \vec{k}\right)$, nas expressões para o núcleo dos operadores em coordenadas relativas,

$$
\begin{aligned}
\hat{M}(\vec{\xi}, \vec{\eta})= & \hat{M}_{0}(\vec{\xi}, \vec{\eta})+\frac{1}{4}(b+2) \kappa^{2} \hat{M}_{0}(\vec{\xi}, \overrightarrow{0})\left(\frac{1}{2} P-P_{1}\right) \hat{M}(\overrightarrow{0}, \vec{\eta}) \\
& +\sum_{i=1}^{2} \frac{1}{3} \kappa^{2} \hat{M}_{0}\left(\vec{\xi}, e^{i}\right) P_{-} \hat{M}\left(e^{i}, \vec{\eta}\right) .
\end{aligned}
$$

$P_{-}$é dado por

$$
P_{-}=\left(\begin{array}{ll}
0 & 0 \\
0 & 1
\end{array}\right)
$$

Re-escalonando a eq. (4.19) por $\kappa^{2} / 2$ obtemos:

$$
\begin{aligned}
\hat{S}(\vec{\xi}, \vec{\eta})= & \hat{S}_{0}(\vec{\xi}, \vec{\eta})+\frac{1}{2}(b+2) \hat{S}_{0}(\vec{\xi}, \overrightarrow{0})\left(\frac{1}{2} P-P_{1}\right) \hat{S}(\overrightarrow{0}, \vec{\eta}) \\
& +\sum_{i=1}^{2} \frac{2}{3} \hat{S}_{0}\left(\vec{\xi}, e^{i}\right) P_{-} \hat{S}\left(e^{i}, \vec{\eta}\right) .
\end{aligned}
$$

onde $\hat{S}_{0}=\left(\kappa^{2} / 2\right) \hat{M}_{0}$ e $\hat{S}=\left(\kappa^{2} / 2\right) \hat{M}$. A eq. (4.20) será usada na próxima seção na determinação do estado ligado.

\subsection{Solução da equação de Bethe-Salpeter na aproximação escada para $K$}

Nesta seção, apresentamos a solução da equação de B-S na aproximação escada. Antes, no entanto, apresentaremos a representação para $\hat{S}_{0}(\vec{\xi}, \vec{\eta})$ em analogia com a eq. (3.31). Primeiramente, consideramos $\hat{M}_{0}$. Relembrando, da eq. (4.10) na Seção 4.1, obtemos para $\hat{M}_{0}$ a seguinte estrutura matricial

$$
\hat{M}_{0}(\vec{\xi}, \vec{\eta})=\left(\begin{array}{cc}
\hat{D}_{0,1111}(\vec{\xi}, \vec{\eta}) & 0 \\
0 & \hat{D}_{0,2222}(\vec{\xi}, \vec{\eta})
\end{array}\right)
$$

Procedendo como na seção 3.3 , obtemos a representação para $\hat{D}_{0, l_{1} l_{2} l_{3} l_{4}}\left(\vec{\xi}, \vec{\eta}, k^{0}\right)$, similar à eq. (3.30), 


$$
\begin{aligned}
& \hat{D}_{0, l_{1} l_{2} l_{3} l_{4}}\left(\vec{\xi}, \vec{\eta}, k^{0}\right)=(2 \pi)^{2} \int_{-1}^{1} \int_{-1}^{1} \int_{\mathbf{T}^{2}} f\left(k^{0}, \lambda^{0} \lambda^{\prime 0}\right)\{[\cos \vec{p} \cdot \vec{\xi} \cos \vec{p} \cdot \vec{\eta}+\operatorname{sen} \vec{p} \cdot \vec{\xi} \operatorname{sen} \vec{p} \cdot \vec{\eta}] \\
& \times \delta_{l_{1} l_{3}} \delta_{l_{2} l_{4}} d \sigma_{\vec{p}, l_{1} l_{3}}\left(\lambda^{0}\right) d \sigma_{\vec{p}, l_{2} l_{4}}\left(\lambda^{\prime 0}\right)+[\cos \vec{p} \cdot \vec{\xi} \cos \vec{p} \cdot \vec{\eta}-\operatorname{sen} \vec{p} \cdot \vec{\xi} \operatorname{sen} \vec{p} \cdot \vec{\eta}] \\
& \left.\times \delta_{l_{1} l_{4}} \delta_{l_{2} l_{3}} d \sigma_{\vec{p}, l_{1} l_{4}}\left(\lambda^{0}\right) d \sigma_{\vec{p}, l_{2} l_{3}}\left(\lambda^{\prime 0}\right)\right\} d \vec{p}+(2 \pi)^{-2} \int_{\mathbf{T}^{2}}\left\{\tilde{G}_{l_{1} l_{3}}(\vec{p}) G_{l_{2} l_{4}}(\vec{p})[\cos \vec{p} \cdot \vec{\xi}\right. \\
& \left.\times \cos \vec{p} \cdot \vec{\eta}+\operatorname{sen} \vec{p} \cdot \vec{\xi} \operatorname{sen} \vec{p} \cdot \vec{\eta}]+\tilde{G}_{l_{1} l_{4}}(\vec{p}) G_{l_{2} l_{3}}(\vec{p})[\cos \vec{p} \cdot \vec{\xi} \cos \vec{p} \cdot \vec{\eta}-\operatorname{sen} \vec{p} \cdot \vec{\xi} \operatorname{sen} \vec{p} \cdot \vec{\eta}]\right\} d \vec{p},
\end{aligned}
$$

onde $f(x, y)$ é o mesmo da Proposição 1 e $d \sigma_{\vec{p}, k l}\left(\lambda^{0}\right)$ admite a decomposição

$$
d \sigma_{\vec{p}, k l}\left(\lambda^{0}\right)=Z_{k l}(\vec{p}) \delta\left(\lambda^{0}-e^{-\omega_{k l}(\vec{p})}\right) d \lambda^{0}+d \hat{\sigma}_{\vec{p}, k l}\left(\lambda^{0}\right) .
$$

Aqui $k l=11$ coresponde a $I=0$ e $k l=22,33,44$ corresponde a $I=1$. Conforme discutimos na Seção 3.3 , a contribuição dominante para $\hat{D}_{0,1111}(\vec{\xi}, \vec{\eta})$ e $\hat{D}_{0,2222}(\vec{\xi}, \vec{\eta})$ vem, se desprezarmos a parte independente de $k^{0}$ e cosiderarmos a contribuição de uma partícula, i.e., fazemos a aproximação na medida espectral $d \sigma_{\vec{p}, I}\left(\lambda^{0}\right) \approx Z_{I}(\vec{p}) \delta\left(\lambda^{0}-e^{-\omega_{I}(\vec{p})}\right) d \lambda^{0}$. Desta forma, obtemos as expressões para $\hat{D}_{0,1111}(\vec{\xi}, \vec{\eta})$ e $\hat{D}_{0,2222}(\vec{\xi}, \vec{\eta})$

$$
\hat{D}_{0,1111}(\vec{\xi}, \vec{\eta}) \simeq(2 \pi)^{-2} \int_{\mathbf{T}^{2}} \frac{\cos \vec{p} \cdot \vec{\xi} \cos \vec{p} \cdot \vec{\eta}}{e^{2\left(\omega_{0}(\vec{p})-m_{0}\right)+\bar{\epsilon}}-1} d \vec{p}
$$

e

$$
\hat{D}_{0,2222}(\vec{\xi}, \vec{\eta}) \simeq(2 \pi)^{-2} \int_{\mathbf{T}^{2}} \frac{\cos \vec{p} \cdot \vec{\xi} \cos \vec{p} \cdot \vec{\eta}}{e^{2\left(\omega_{1}(\vec{p})-m_{1}\right)+\bar{\epsilon}}-1} d \vec{p}
$$

lembrando que até a ordem $\mathcal{O}\left(\kappa^{3}\right)$ as massas e curvas de dispersão para os mésons com isospin total $I=0,1$ coincidem obtemos

$$
\hat{D}_{0} \equiv \hat{D}_{0,1111}(\vec{\xi}, \vec{\eta}) \simeq \hat{D}_{0,2222}(\vec{\xi}, \vec{\eta}) \simeq(2 \pi)^{-2} \int_{\mathbf{T}^{2}} \frac{\cos \vec{p} \cdot \vec{\xi} \cos \vec{p} \cdot \vec{\eta}}{2 c^{m} \kappa^{2}[-\tilde{\Delta}(\vec{p})]+\bar{\epsilon}} d \vec{p}
$$

com $c^{m}$ o mesmo da eq. (3.5). Notamos que na aproximação dada pela eq. (4.21) $\hat{D}_{0}$ é o mesmo da eq. (3.31). Lembrando que $\hat{S}=\left(\kappa^{2} / 2\right) \hat{D}$, deste ponto em diante, usamos a notação compacta $\hat{S}_{0,1111} \simeq \hat{S}_{0,2222} \equiv \hat{R}_{0}$. Desta forma, como no capítulo anterior, fazendo as identificações $\hat{R}_{0}(\overrightarrow{0}, \overrightarrow{0})=r_{00}, \hat{R}_{0}\left(\overrightarrow{0}, e^{1}\right)=r_{01}, \hat{R}_{0}\left(e^{1}, e^{1}\right)=r_{11}$, e $\hat{R}_{0}\left(e^{1}, e^{2}\right)=r_{12}$, então, as seguintes relações valem

$$
r_{01}=r_{02}, r_{11}=r_{22}, r_{01}=r_{10}, r_{01}=-\frac{1}{2}+\frac{1}{2}(2+b) r_{00} \text { e } r_{11}+r_{12}=(2+b) r_{01} .
$$

Resolvendo a eq. (4.20) para as matrizes $2 \times 2 \hat{S}(0, \vec{\eta}), \hat{S}\left(e^{i}, \vec{\eta}\right),(i=1,2)$ e usando as 
relações (4.22), vemos que as únicas possíveis singularidades de $\hat{S}$, que são as mesmas de $\hat{M}$, vêm dos zeros do determinante da matriz $6 \times 6$, que chamaremos de $\mathcal{W}$, i.e.

$$
\mathcal{W}=\left(\begin{array}{rrr}
I_{2}-\frac{b+2}{2} r_{00} Q & -\frac{2}{3} r_{01} P_{-} & -\frac{2}{3} r_{01} P_{-} \\
-\frac{b+2}{2} r_{01} Q & I_{2}-\frac{2}{3} r_{11} P_{-} & -\frac{2}{3} r_{12} P_{-} \\
-\frac{b+2}{2} r_{01} Q & -\frac{2}{3} r_{12} P_{-} & I_{2}-\frac{2}{3} r_{11} P_{-}
\end{array}\right)
$$

onde

$$
Q=\frac{1}{2} P-P_{1}=\frac{1}{8}\left(\begin{array}{rr}
-5 & 3 \sqrt{3} \\
3 \sqrt{3} & 1
\end{array}\right) .
$$

Segue, então, que det $\mathcal{W}$ tem a estrutura

$$
\operatorname{det} \mathcal{W}=\left|\begin{array}{rrrrrr}
1+a_{1} & a_{2} & 0 & 0 & 0 & 0 \\
a_{2} & 1+a_{3} & 0 & a_{4} & 0 & a_{4} \\
a_{5} & a_{6} & 1 & 0 & 0 & 0 \\
a_{6} & a_{7} & 0 & 1+a_{8} & 0 & a_{9} \\
a_{5} & a_{6} & 0 & 0 & 1 & 0 \\
a_{6} & a_{7} & 0 & a_{9} & 0 & 1+a_{8}
\end{array}\right|
$$

com

$$
\begin{gathered}
a_{1}=\frac{5}{16}(2+b) r_{00}, \quad a_{2}=-\frac{3 \sqrt{3}}{16}(2+b) r_{00}, \quad a_{3}=-\frac{1}{16}(2+b) r_{00}, \\
a_{4}=-\frac{2}{3} r_{01} \quad a_{5}=\frac{5}{16}(2+b) r_{01}, \quad a_{6}=-\frac{3 \sqrt{3}}{16}(2+b) r_{01}, \\
a_{7}=-\frac{1}{16}(2+b) r_{01}, \quad a_{8}=-\frac{2}{3} r_{11}, \quad a_{9}=-\frac{2}{3} r_{12} .
\end{gathered}
$$

Usando a expansão de Laplace na terceira e quinta coluna do determinante acima obtemos que $\operatorname{det} \mathcal{W}$ pode ser reescrito da seguinte forma

$$
\operatorname{det} \mathcal{W}=\left|\begin{array}{rrrr}
1+a_{1} & a_{2} & 0 & 0 \\
a_{2} & 1+a_{3} & a_{4} & a_{4} \\
a_{6} & a_{7} & 1+a_{8} & a_{9} \\
a_{6} & a_{7} & a_{9} & 1+a_{8}
\end{array}\right| .
$$

Desta forma, obtemos as seguintes condições para a existência do estado ligado:

$$
\begin{gathered}
1+a_{8}-a_{9}=0 . \\
{\left[\left(1+a_{1}\right)\left(1+a_{3}\right)-a_{2}^{2}\right]\left(1+a_{8}+a_{9}\right)+2 c=0}
\end{gathered}
$$


com

$$
c=a_{4}\left[a_{2} a_{6}-\left(1+a_{1}\right) a_{7}\right] .
$$

De agora em diante usaremos as notações compactas

$$
C_{1} \equiv 1+a_{8}-a_{9} \quad \text { e } C_{2} \equiv\left[\left(1+a_{1}\right)\left(1+a_{3}\right)-a_{2}^{2}\right]\left(1+a_{8}+a_{9}\right)+2 c .
$$

Com relação à (4.27) temos:

$$
C_{1}=1-\frac{2}{3}\left(r_{11}-r_{12}\right)
$$

que agora analisamos. Como $\left(r_{11}-r_{12}\right)$ é uma função monótona decrescente de $b$ é suficiente verificarmos que $\frac{2}{3}\left(r_{11}-r_{12}\right)<1$ para $b=0$. Para verificarmos isto dividimos o intervalo de integração em $\left(r_{11}-r_{12}\right)$ em três setores usando a resolução da identidade $\left[\chi_{(0, a)}(x)+\chi_{(a, \pi)}(x)\right]\left[\chi_{(0, a)}(y)+\chi_{(a, \pi)}(y)\right] \equiv 1$. Desta forma, obtemos:

$$
\begin{aligned}
r_{11}-r_{12}= & \frac{2}{(2 \pi)^{2}} \int_{(0, a)^{2}} \frac{\left(\cos p_{1}-\cos p_{2}\right)^{2}}{\left(2-\cos p_{1}-\cos p_{2}\right)} d \vec{p} \\
& +\frac{4}{(2 \pi)^{2}} \int_{(0, a)} \int_{(a, \pi)} \frac{\left(\cos p_{1}-\cos p_{2}\right)^{2}}{\left(2-\cos p_{1}-\cos p_{2}\right)} d \vec{p} \\
& +\frac{2}{(2 \pi)^{2}} \int_{(a, \pi)^{2}} \frac{\left(\cos p_{1}-\cos p_{2}\right)^{2}}{\left(2-\cos p_{1}-\cos p_{2}\right)} d \vec{p} .
\end{aligned}
$$

Sejam $A, B$ e $C$ uma notação para o primeiro, segundo e terceiro termo, respectivamente, que aparecem no lado direito da equação anterior. As duas últimas integrais, $B$ e $C$, podem ser calculadas numericamente para $a$ suficientemente pequeno, por exemplo, $a=10^{-5}$. Finalmente:

$$
B=0,3183 \times 10^{-5} \text { e } C=0,2732 .
$$

A terceira integral pode ser avaliada fazendo a aproximação $\cos x \approx 1-x^{2} / 2$ para $x \ll 1$ :

$$
A=\mathcal{O}\left(a^{4}\right) \approx 0
$$

Desta forma obtemos $(2 / 3)(A+B+C)=0,1821<1$, portanto excluindo estados ligados do espectro.

Para (4.28) temos:

$$
\left[\left(1+a_{1}\right)\left(1+a_{3}\right)-a_{2}^{2}\right]=1+\frac{1}{4}(2+b) r_{00}-\frac{1}{8}(2+b)^{2} r_{00}^{2}
$$

e

$$
c=a_{4}\left[a_{2} a_{6}-\left(1+a_{1}\right) a_{7}\right]=-\frac{1}{12} r_{01}^{2}(2+b)\left[1+2(2+b) r_{00}\right] .
$$


Desta forma obtemos para (4.28)

$$
C_{2}=\left[1+\frac{1}{4}(2+b) r_{00}-\frac{1}{8}(2+b)^{2} r_{00}^{2}\right]\left[1-\frac{2}{3}\left(r_{11}+r_{12}\right)\right]-\frac{1}{6} r_{01}^{2}(2+b)\left[1+2(2+b) r_{00}\right] .
$$

Observando que podemos escrever $r_{11}+r_{12}=(2+b) r_{01}$ e $r_{01}=-\frac{1}{2}+\frac{1}{2}(2+b) r_{00}$ obtemos

$$
\begin{aligned}
C_{2}= & {\left[1+\frac{1}{4}(2+b) r_{00}-\frac{1}{8}(2+b)^{2} r_{00}^{2}\right]\left\{1-\frac{2}{3}(2+b)\left[-\frac{1}{2}+\frac{1}{2}(2+b) r_{00}\right]\right\} } \\
& -\frac{1}{6}\left[-\frac{1}{2}+\frac{1}{2}(2+b) r_{00}\right]^{2}(2+b)\left[1+2(2+b) r_{00}\right] .
\end{aligned}
$$

Esta última expressão fornece a condição para o estado ligado

$$
C_{2}=c_{0}+c_{1} r_{00}+c_{2} r_{00}^{2}+c_{3} r_{00}^{3}=0
$$

com

$$
\begin{gathered}
c_{0}=1+(2+b) \frac{5}{16}=\frac{13}{8}+\frac{5 b}{16} \\
c_{1}=-\frac{1}{4}(2+b)(1+b) \\
c_{2}=-\frac{1}{16}(2+b)^{2}(4+b) \\
c_{3}=0 .
\end{gathered}
$$

Fatorando a eq. (4.29), polinômio de segundo grau em $r_{00}$, obtemos a seguinte condição para a existência do estado ligado, com $g(b) \equiv 12+6 b+b^{2}$

$$
r_{00}=\frac{-c_{1} \pm \sqrt{c_{1}^{2}-4 c_{2} c_{0}}}{2 c_{2}}=\frac{-2(1+b) \pm \sqrt{g(b)}}{(2+b)(4+b)} \equiv r_{ \pm}
$$

o caso $r_{00}=r_{+}$é excluído, já que, é uma raiz negativa e $r_{00} \geq 0$. Finalmente:

$$
r_{00}=\frac{-2(1+b)+\sqrt{g(b)}}{(2+b)(4+b)}
$$

Para analisarmos a raiz positiva $r_{-}$, notamos que

$$
r_{00}=(2 \pi)^{-2} \int_{\mathbf{T}^{2}} \frac{1}{2-\cos p^{1}-\cos p^{2}+b} d \vec{p}
$$

é positiva, monótona decrescente, analítica em $b>0$, com singularidade logarítimica em $b=0$. Ademais, notamos que para $b>0, r_{00}$ admite uma representação em termos de funções elíticas. $r_{00}$ é, a menos de um fator, a transformada de Fourier do quadrado da função de Bessel $J_{0}$, 
desta forma temos a representação para $r_{00}$

$$
r_{00}=\frac{2}{\pi(b+2)} K\left(\frac{2}{b+2}\right)
$$

onde $K(a) \equiv \int_{0}^{\pi / 2}\left(1-a^{2} \operatorname{sen}^{2} \phi\right) d \phi, 0 \leq a \leq 1$. E nós notamos pela eq. (4.30) que o comportamento assintótico para $b$ grande de $r_{00}$ é dado por $1 /(b+2)$, observando que, neste caso, $K(2 /(b+2)) \sim K(0)=\pi / 2$. A raiz

$$
r_{-}=\frac{-2(b+1)+3 \sqrt{g(b)}}{(b+2)(b+4)}
$$

é $r_{-}(b) \simeq-1 / 4+3 \sqrt{3} / 4+\mathcal{O}(b) \simeq 1,04904+\mathcal{O}(b)$, para $b$ pequeno, é real analítica, decresce monotonicamente e comporta assintoticamente como $1 / b$, para $b$ grande. Desta forma, o gráfico de $r_{00}$ está acima do gráfico de $r_{-}$para $b=0$ e, para $b$ grande notamos que está abaixo (pelo comportamento assintótico para $b$ grande, lembrando que $r_{00} \sim 1 /(b+2)$ e $r_{-} \sim 1 / b$ ). Assim, existe uma existe uma solução para a equação

$$
r_{-}=(2 \pi)^{-2} \int_{\mathbf{T}^{2}} \frac{1}{2-\cos p^{1}-\cos p^{2}+b} d \vec{p}
$$

Graficamente a solução $\bar{b}$ para eq. (4.32) é única. Um cálculo numérico fornece $\bar{b} \simeq 0,02359$, desta forma o estado ligado tem uma energia de ligação da ordem $\bar{\epsilon}=-\ln \left(1-\bar{b} \kappa^{2}\right) \approx$ $\bar{b} \kappa^{2}=0,02359 \kappa^{2}$, que, conforme mencionamos na Observação 8 é consistente com nossas aproximações para a equação de B-S, i.e., a aproximação $L$ para $K$ dada pela eq. (4.18).

Uma questão importante é entender como as partes atrativas contrabalançam com as partes repulsivas na eq. (4.18) de tal forma que o estado ligado ainda apareça. Para isto, resolvemos numericamente o problema considerando somente o efeito das contribuições (I) e (III), e negligenciando o termo de troca (II). A equação resultante é dada por

$$
\hat{S}(\vec{\xi}, \vec{\eta})=\hat{S}_{0}(\vec{\xi}, \vec{\eta})+\frac{1}{2}(b+2) \hat{S}_{0}(\vec{\xi}, \overrightarrow{0})\left(\frac{1}{2} P-P_{1}\right) \hat{S}(\overrightarrow{0}, \vec{\eta})
$$

Resolvendo esta equação para a matriz $2 \times 2 \hat{S}(\overrightarrow{0}, \vec{\eta})$ obtemos, neste caso, a condição para o estado ligado

$$
\operatorname{det} \mathcal{W}^{\prime}=\left|\begin{array}{rr}
1+a_{1} & a_{2} \\
a_{2} & 1+a_{3}
\end{array}\right|=-\frac{1}{8}\left[(b+2) r_{00}-4\right]\left[(b+2) r_{00}+2\right]=0,
$$

com $a_{1}, a_{2}$ e $a_{3}$ definidos na eq. (4.24). Desta forma obtemos a condição para o estado ligado, $r_{00}=4 /(b+2)$. Notamos ainda que como $r_{00} \geq 0$ temos $(b+2) r_{00}+2>0$. Aqui, a quantidade $r_{-}$da eq. (4.31) é substituida por $r^{\prime} \equiv 4 /(b+2)$ e, ao invés da eq. (4.32), obtemos a condição 
$r_{00}=r^{\prime}$ que fornece a solução $b^{\prime} \simeq 0,00006$. Conforme esperado, como a atração é reduzida neste caso, temos $b^{\prime}<\bar{b}$, e uma energia de ligação consideravelmente menor.

Para ver como o potencial atrativo associado com a contribuição de troca de um quasiméson (II) compara a parte atrativa do potencial (I) e (III), também resolvemos o problema considerando somente a contribuição (II) e negligenciando (I) e (III). Para este caso, obtemos a seguinte equação

$$
\hat{S}(\vec{\xi}, \vec{\eta})=\sum_{i=1}^{2} \frac{2}{3} \hat{S}_{0}\left(\vec{\xi}, e^{i}\right) P_{-} \hat{S}\left(e^{i}, \vec{\eta}\right)
$$

Resolvendo esta equação para as matrizes $2 \times 2 \hat{S}\left(e^{i}, \vec{\eta}\right),(i=1,2)$, a condição para o estado ligado é dada por

$$
\operatorname{det} \mathcal{W}^{\prime \prime}=\left|\begin{array}{rrrr}
1 & 0 & 0 & 0 \\
0 & 1+a_{8} & 0 & a_{9} \\
0 & 0 & 1 & 0 \\
0 & a_{9} & 0 & 1+a_{8}
\end{array}\right|=\left|\begin{array}{rr}
1+a_{8} & a_{9} \\
a_{9} & 1+a_{8}
\end{array}\right|=1-\frac{2}{3}\left(r_{11}+r_{12}\right)
$$

e usando as relações (3.36) e (3.37) obtemos a condição para o estado ligado neste caso

$$
\operatorname{det} \mathcal{W}^{\prime \prime}=\frac{b+5}{3}-\frac{(b+2)^{2}}{3} r_{00}=0
$$

Desta forma, ao invés de $r_{-}$na eq. (4.31), temos $r^{\prime \prime}=(b+5) /(b+2)^{2}$. Conforme esperado, $r_{00}=(b+5) /(b+2)^{2}$ tem solução, dada por $b^{\prime \prime} \simeq 0,00639$.

Desta forma, obtemos $b^{\prime}<b^{\prime \prime}<\bar{b}$, e nossa análise mostra: que a parte atrativa no potencial efetivo nos casos (I) e (III) dominam sua parte repulsiva; que a atração associada ao caso (II), i.e. a troca de um quasi-méson, substancilamente aumenta a ligação. Na figura 4.1 nós apresentamos as condições para o estado ligado nos três casos mencionados, $b^{\prime}, b^{\prime \prime}$ e $\bar{b}$.

Para que tenhamos uma idéia melhor do balanceamento entre as partes atrativas e repulsivas, analisamos o efeito de correlações de calibre no caso (III). Ou seja, ao invés de usar a integral $\mathcal{I}_{4}$ da eq. (A.12), nós erronêamente usamos a contribuição não correlacionada $\mathcal{I}_{2}^{2}$, onde $\mathcal{I}_{2}$ é dado pela eq. (A.10). O efeito desta troca é de substituir o operador $\left(P_{1} / 4-P / 8\right)$ por $-I_{2} / 4$, no termo (III). Com isto, não existe mais a recombinação dos termos (I) e (III) e, consequentemente, ambos termos são da ordem $\kappa^{0}$. Quando efetuamos o re-escalonamento pelo fator $\kappa^{2} / 2$ [veja eq. (4.20)], o potencial associado com estes dois termos adquire um fator multiplicativo $2 / \kappa^{2}$. Como $0<\kappa<<1$, isto significa um grande coeficiente. Para vermos o efeito da correlação de campos de calibre, novamente resolvemos o problema. A equação resultante neste caso é dada por

$$
\hat{S}(\vec{\xi}, \vec{\eta})=\hat{S}_{0}(\vec{\xi}, \vec{\eta})-\frac{1}{\kappa^{2}} \hat{S}_{0}(\vec{\xi}, \overrightarrow{0})\left(\frac{1}{4} P+P_{1}\right) \hat{S}(\overrightarrow{0}, \vec{\eta})+\sum_{i=1}^{2} \frac{2}{3} \hat{S}_{0}\left(\vec{\xi}, e^{i}\right) P_{-} \hat{S}\left(e^{i}, \vec{\eta}\right)
$$


Resolvendo a eq. (4.35) para as matrizes $\hat{S}(\overrightarrow{0}, \vec{\eta})$ e $\hat{S}\left(e^{i}, \vec{\eta}\right)(i=1,2)$ obtemos que a condição para o estado ligado, neste caso, é dada pelos zeros do determinante $\operatorname{det} W^{\prime}$ onde

$$
W^{\prime}=\left(\begin{array}{rrr}
I_{2}+\frac{1}{\kappa^{2}} r_{00} Q^{\prime} & -\frac{2}{3} r_{01} P_{-} & -\frac{2}{3} r_{01} P_{-} \\
\frac{1}{\kappa^{2}} r_{01} Q^{\prime} & I_{2}-\frac{2}{3} r_{11} P_{-} & -\frac{2}{3} r_{12} P_{-} \\
\frac{1}{\kappa^{2}} r_{01} Q^{\prime} & -\frac{2}{3} r_{12} P_{-} & I_{2}-\frac{2}{3} r_{11} P_{-}
\end{array}\right)
$$

onde, neste caso,

$$
Q^{\prime}=\left(\frac{1}{4} P+P_{1}\right)=\frac{1}{8}\left(\begin{array}{rr}
13 & -3 \sqrt{3} \\
-3 \sqrt{3} & 7
\end{array}\right) .
$$

Desta forma, obtemos:

$$
\operatorname{det} W^{\prime}=\left|\begin{array}{rrrrrr}
1+a_{1}^{\prime} & a_{2}^{\prime} & 0 & 0 & 0 & 0 \\
a_{2}^{\prime} & 1+a_{3}^{\prime} & 0 & a_{4} & 0 & a_{4} \\
a_{5}^{\prime} & a_{6}^{\prime} & 1 & 0 & 0 & 0 \\
a_{6}^{\prime} & a_{7}^{\prime} & 0 & 1+a_{8} & 0 & a_{9} \\
a_{5}^{\prime} & a_{6}^{\prime} & 0 & 0 & 1 & 0 \\
a_{6}^{\prime} & a_{7}^{\prime} & 0 & a_{9} & 0 & 1+a_{8}
\end{array}\right| .
$$

com

$$
\begin{array}{ll}
a_{1}^{\prime}=\frac{13}{8} \frac{r_{00}}{\kappa^{2}}, & a_{2}^{\prime}=-\frac{3 \sqrt{3}}{8} \frac{r_{00}}{\kappa^{2}}, \quad a_{3}^{\prime}=\frac{7}{8} \frac{r_{00}}{\kappa^{2}}, \\
a_{5}^{\prime}=\frac{13}{8} \frac{r_{01}}{\kappa^{2}}, & a_{6}^{\prime}=-\frac{3 \sqrt{3}}{8} \frac{r_{01}}{\kappa^{2}}, \quad a_{7}^{\prime}=\frac{7}{8} \frac{r_{01}}{\kappa^{2}},
\end{array}
$$

e os elementos restantes foram definidos anteriormente nas eqs. (4.25) e (4.26).

Expandindo o determinante acima como fizemos anteriormente obtemos as seguintes condições para o estado ligado:

$$
C_{1}^{\prime} \equiv C_{1}=1-\frac{2}{3}\left(r_{11}-r_{12}\right)=0
$$

$\mathrm{e}$

$$
C_{2}^{\prime} \equiv\left[\left(1+a_{1}^{\prime}\right)\left(1+a_{3}^{\prime}\right)-\left(a_{2}^{\prime}\right)^{2}\right]\left(1+a_{8}+a_{9}\right)+2 c^{\prime}=0
$$

onde

$$
c^{\prime}=a_{4}\left[a_{2}^{\prime} a_{6}^{\prime}-\left(1+a_{1}^{\prime}\right) a_{7}^{\prime}\right] .
$$

Para a eq. (4.38) obtemos a condição para o estado ligado

$$
C_{2}^{\prime}=c_{0}+c_{1} r_{00}+c_{2} r_{00}^{2}+c_{3} r_{00}^{3}=0
$$


com

$$
\begin{gathered}
c_{0}=1+\frac{b+2}{3}+\frac{7}{24} \frac{1}{\kappa^{2}} \\
c_{1}=-\frac{(b+2)^{2}}{3}+\frac{5}{2} \frac{1}{\kappa^{2}}+\frac{(b+2)}{4} \frac{1}{\kappa^{2}}+\frac{1}{3} \frac{1}{\kappa^{4}} \\
c_{2}=\frac{7(b+2)^{2}}{24} \frac{1}{\kappa^{2}}+\frac{1}{\kappa^{4}}-\frac{(b+2)}{3} \frac{1}{\kappa^{4}} \\
c_{3}=0 .
\end{gathered}
$$

Notemos primeiramente que, a condição $C_{1}^{\prime}$, dada pela eq. (4.37) foi analisada anteriormente. Para a eq. (4.38) no limite $\kappa \rightarrow 0$ os termos dominantes para $c_{0}, c_{1}$ e $c_{2}$ são

$$
c_{0} \simeq \frac{7}{24} \frac{1}{\kappa^{2}}, \quad c_{1} \simeq \frac{1}{3} \frac{1}{\kappa^{4}} \text { e } c_{2} \simeq \frac{1}{\kappa^{4}}-\frac{(b+2)}{3} \frac{1}{\kappa^{4}}
$$

e desta forma obtemos

$$
r_{00}=\frac{-c_{1} \pm \sqrt{c_{1}^{2}-4 c_{2} c_{0}}}{2 c_{2}} \simeq-\frac{c_{1}}{c_{2}} \simeq \frac{1}{b-1}
$$

Vemos então que para a segunda condição, dada pela eq. (4.38), obtemos uma condição como a eq. (4.31) para a energia de ligação com $r_{-}$substituído por $1 /(b-1)$, no limite $\kappa \rightarrow 0$. Como temos a cota rigorosa $r_{00}>1 /(b+2)$ (que segue da representação de $r_{00}$ em termos de funções elíticas), vemos que não existe um estado ligado mesmo incluindo a contribuição atrativa da troca de um quasi-méson. Podemos entender este fato no contexto da equação resolvente de Schrödinger na rede. Consideramos o operador de Schrödinger, em $\ell_{2}\left(\mathbb{Z}^{2}\right),(-\Delta / 2)$ menos o potencial $V_{0}+\lambda V_{1}$, onde $V_{0}$ é um potencial repulsivo na origem que mandamos para $+\infty$ (correspondendo a tomar $\kappa \rightarrow 0$ ), fornecendo uma condição de Dirichlet na origem. $\lambda V_{1}$ é um potencial de posto um e atrativo. A condição para o estado ligado que obtemos é dada pela eq. (4.31), mas com $r_{-}$substituído por $\breve{r} \equiv[b+2(1-1 / \lambda)]^{-1}$. Para $\lambda$ positivo, esta equação tem solução somente para $\lambda$ acima de um valor crítico $\breve{\lambda}$. Uma análise numérica mostra que $\breve{\lambda}$ é aproximadamente da ordem $\breve{\lambda} \simeq 2,5$. 


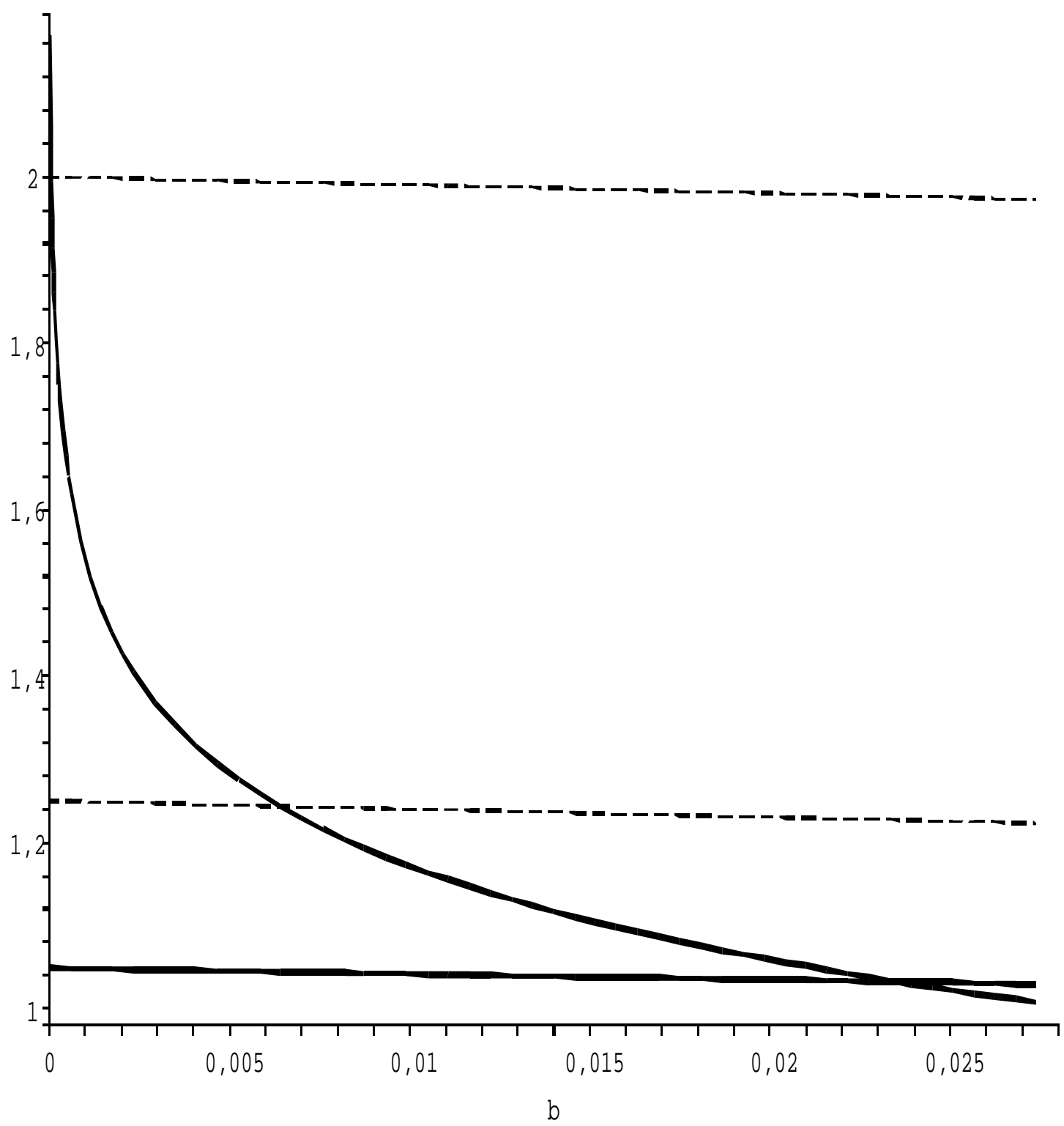

Figura 4.1: Gráficos relativos à condição de estado ligado como funções do parâmetro $b$. A curva sólida que diverge em $b=0$ é $r_{00}$. A curva horizontal inferior corresponde a $r_{-}$. A curva pontilhada superior é $r^{\prime}$ e a mais inferior é $r^{\prime \prime}$. As coordenadas de $b$ nos pontos de interseção com $r_{00}$ são dadas por: para $r_{-}, b=\bar{b} \simeq 0,02359$; para $r^{\prime}, b=b^{\prime} \simeq 0$, 00006; para $r^{\prime \prime}, b=b^{\prime \prime} \simeq 0,00639$. 


\section{Capítulo 5}

\section{Conclusão}

Neste trabalho, a partir de primeiros princípios, usando a formulação Euclideana de TQC, e usando resultados preliminares a respeito da existência de bárions [11, 12], discutimos o espectro de energia-momento em QCD na rede no regime de acoplamento forte. Neste contexto, trabalhando no regime de acoplamento forte e na aproximação escada, obtivemos os seguintes resultados:

No Capítulo 2, fizemos uma análise do padrão espectral de uma partícula no setor mesônico em $(2+1)$ e $(3+1)$ dimensões, um sabor e usando matrizes de Dirac $4 \times 4$. Um resultado essencial deste capítulo e usado ao longo desta tese é a apresentação de uma representação espectral geral para vetores em $\mathcal{H} \supset \mathcal{H}_{e}, \mathcal{H}_{o}$. É esta representação que nos permite relacionar o espectro de energia-momento com singularidades de determinadas funções de correlação (no nosso caso dois e quatro pontos) e garantir que, realmente, estamos obtendo curvas de dispersão e massas. A análise do espectro de uma partícula constitui-se num passo fundamental para a análise de estados ligados com várias partículas do modelo.

No Capítulo 3, considerando o espectro até o limiar de duas partículas, mostramos a ausência de estados ligados do tipo méson-méson e méson-bárion em $(2+1)$ dimensões, um sabor e usando matrizes de spin $2 \times 2$. A partir da equação de B-S na aproximação escada (ordem dominante no parâmetro de "hopping" $\kappa$ ) mostramos que não existem estados ligados de méson-méson e bárion-méson abaixo do limiar de dois mésons $(-4 \ln \kappa)$ e de um méson e um bárion $(-5 \ln \kappa)$. Interpretamos este efeito como consequência da exclusão de Pauli entre os quarks, já que o espaço de configuração dos campos de quarks é demais pequeno devido ao baixo número de graus de liberdade de spin e sabor.

No Capítulo 4, analisamos a existência de estados ligados de dois mésons, com dois sabores (caso com isospin), matrizes de spin $2 \times 2$ usando a mesma abordagem do Capítulo 3, i.e., a equação de B-S na aproximação escada. Intuitivamente, a adição de um grau de liberdade interno extra diminui o efeito da exclusão de Pauli aumentando a chance de duas partículas se ligarem. Na aproximação escada obtivemos que a interação dominante entre dois 
mésons é descrita por um potencial com dois tipos de contribuições, uma delas para pontos coincidentes, dependente da energia e com partes atrativa e repulsiva; e outra, de alcance espacial um, atrativa, relacionada com a troca de um quasi-méson (o par quark-antiquark envolvido não apresenta os índices corresto de spin de um méson). Restringindo a nossa atenção ao setor de isospin total zero, como em [15] onde existe um estado ligado bárionbárion, nós detectamos um estado ligado méson-méson com energia de ligação da ordem de $0,02359 \kappa^{2}$. A fim de entendermos o mecanismo de atração entre os mésons, estudamos o efeito de correlação numa integral de calibre relevante para a nossa análise do potencial de alcance zero. Ao considerar erroneamente uma integral com quatro campos de calibre $\mathcal{I}_{4}$ (veja a eq. A.12) como produto descorrelacionado de duas integrais de calibre com apenas dois campos de calibre tal qual $\mathcal{I}_{2}$ (veja a eq. (A.10)) no cálculo da aproximação escada, esta troca transforma nosso potencial atrativo em um potencial repulsivo, acarretando o desaparecimento no espectro deste estado ligado méson-méson. A partir daí concluímos que a correlação entre integrais de calibre desempenha um papel importante na existência do estado ligado. Para ainda termos um maior entendimento dos mecanismos de atração/repulsão se desconsiderarmos a interação de troca de um quasi-méson e ainda verificamos a existência deste estado ligado méson-méson. A energia de ligação neste caso é bem menor, conforme esperado, já que a parte atrativa vinda da troca de quasi-mésons foi desconsiderada. Da mesma forma, ao desligar o potencial dependente da energia (que apresenta partes atrativas e repulsivas), obtivemos neste caso um estado ligado com energia de ligação maior que a do caso anterior, só considerando o potencial de alcance zero. Para o problema envolvendo os dois potenciais obtivemos uma energia de ligação maior que os dois casos anteriores e vemos, então, que estas duas contribuições se combinam de forma a obtermos uma maior energia de ligação. Considerando o potencial associado a troca de quasi-mésons vemos que este resultado corrobora (em um certo sentido) a descrição fenomenológica de Yukawa onde a interação entre as partículas é descrita pela troca de partículas mesônicas. O decaimento exponencial deste potencial (sua taxa) é fixado pela massa do estado ligado.

Nossos resultados abrem caminho para a análise de modelos levando em conta um número maior de graus de liberdade $(3+1$ dimensões e vários sabores). Por exemplo, a existência de estados ligados de prótons e nêutrons, tal como o dêuteron, deve emergir desta análise.

Numa frente complementar de trabalho, já que nosso trabalho na aproximação escada contém os ingredientes necessários (i.e. essencialmente o decaimento desejado para o núcleo de $K$ ), podemos passar à análise do espectro além da aproximação escada.

Muitos são os problemas em aberto. Por exemplo, seria de grande interesse procurar pelo fator polinomial de decaimento na distância $1 / r$ do potencial de Yukawa. Outra extensão interessante seria a inclusão de bósons de Higgs e variáveis de plaquetas. 


\section{Apêndice A}

\section{Fórmula de Creutz para o Cálculo de Integrais de Calibre}

Neste apêndice, introduzimos o método de Creutz para avaliar integrais de calibre [5]. Apesar de não apresentar nenhum procedimento novo (os resultados descritos aqui estão em [5]), incluímos este apêndice com uma finalidade didática. Este método é baseado na construção de uma função geratriz a partir da qual calculamos integrais do tipo

$$
\mathcal{I}=\int d g g_{i_{1} j_{1}} \ldots g_{i_{n} j_{n}} g_{k_{1} \ell_{1}}^{-1} \ldots g_{k_{m} \ell_{m}}^{-1}
$$

onde $d g$ é uma medida de Haar $^{1}$ sobre o grupo de calibre, aqui tomado como $g, g^{-1} \in S U(N)$. Notamos ainda que integrais do tipo

$$
\mathcal{J}=\int d g g_{i_{1} j_{1}} \ldots g_{i_{n} j_{n}}=0
$$

quando $n$ não é múltiplo de três são zero.

Antes de explicarmos detalhadamente a obtenção da fórmula de Creutz vamos dar um sumário do procedimento. O ponto inicial da idéia de Creutz é partir de um funcional gerador que denotaremos por $W$, a partir do qual a integral $\mathcal{I}$ pode ser obtida. A seguir Creutz introduz um Teorema baseado nas propriedades das medidas de Haar, ou seja, a única função analítica que satisfaz a igualdade $W(J)=W\left(g_{0} J g_{1}\right)$ é função de det $J$, ou seja, $W(J)=\sum_{r} a_{r}(\operatorname{det} J)^{r}$. Finalmente, os coeficientes $a_{r}$ são obtidos recursivamente através de um exercício combinatorial.

Neste apêndice adotaremos por simplicidade a convenção de soma sobre índices repetidos, exceto quando mencionamos, explicitamente, o contrário.

\footnotetext{
${ }^{1}$ Dado um grupo de Lie compacto, existe uma única medida com as propriedades $\int d g f(g)=\int d g f\left(g_{0} g g_{1}\right)=$ $\int d g f\left(g^{-1}\right), \int d g=1$ onde $g, g_{0}$ e $g_{1}$ são elementos arbitrários do grupo de Lie.
} 
Vamos agora descrever detalhadamente o procedimento de Creutz. Definindo a função

$$
W(J, K)=\int d g \exp \left[\operatorname{Tr}\left(J g+K g^{-1}\right)\right]
$$

onde $J, K \in \mathbf{M}_{N \times N}(\mathbb{C})$, a integral $\mathcal{I}$ é obtida através da fórmula

$$
\mathcal{I}=\left.\left(\frac{\partial}{\partial J_{j_{1} i_{1}}} \ldots \frac{\partial}{\partial J_{j_{n} i_{n}}} \frac{\partial}{\partial K_{l_{1} k_{1}}} \ldots \frac{\partial}{\partial K_{l_{m} k_{m}}} W(J, K)\right)\right|_{J=K=0} .
$$

Podemos eliminar a dependência em $K$ na eq. (A.2) expressando $g^{-1}$ em termos dos cofatores de $g$. Os cofatores de uma matriz são facilmente obtidos usando o símbolo totalmente antisimétrico $\epsilon_{i_{1} \ldots i_{N}}$ de Levi-Civita.

Como $g$ tem determinante 1 , pois $g \in S U(N)$, temos para $g_{i j}^{-1}$

$$
g_{i j}^{-1}=\frac{1}{\operatorname{det} g}(\operatorname{cof} g)_{j i}=(\operatorname{cof} g)_{j i}=\frac{1}{(N-1) !} \epsilon_{j i_{1} \ldots i_{N-1}} \epsilon_{i j_{1} \ldots j_{N-1}} g_{i_{1} j_{1}} \ldots g_{i_{N-1} j_{N-1}} .
$$

Usando a eq. (A.3) derivadas múltiplas com relação a $J$ podem ser substituídas por derivadas em relação a $K$, desta forma, podemos escrever,

$$
W(J, K)=\left\{e^{\operatorname{Tr}\left[K\left(\operatorname{cof} \frac{\partial}{\partial J}\right)\right]}\right\} W(J),
$$

onde

$$
W(J) \equiv \int d g e^{(\operatorname{Tr} J g)}
$$

Para determinarmos $W(J)$ usamos a propriedade de invariância da medida de Haar, que imediatamente implica em $W(J)=W\left(g_{0} J g_{1}\right)$ onde $g_{0}$ e $g_{1}$ são matrizes arbitrárias de $S U(N)$ e o seguinte Teorema, provado em [7],

Teorema 8 Seja $F(A)$ uma função analítica de $A \in \mathbf{M}_{N \times N}(\mathbb{C})$. Se $F(A)$ satisfaz

$$
F(A)=F\left(g_{0} A g_{1}\right)
$$

com $g_{0}$ e $g_{1}$ elementos arbitrários de $S U(N)$, então $F(A)$ é uma função do determinante de $A$.

Desta forma, podemos escrever

$$
W(J)=\sum_{i=0}^{\infty} a_{i}(\operatorname{det} J)^{i}
$$

Devido à normalização $\int d g=1$, temos $W(J=0)=\int d g=1$ o que implica em $a_{0}=1$. 
Agora, obtemos uma relação de recorrência para os $a_{n}^{\prime} s$. Primeiro, observamos que $W(J)$ satisfaz à seguinte equação diferencial

$$
\left(\operatorname{det} \frac{\partial}{\partial J}\right) W(J)=W(J)
$$

obtida lembrando que

$$
\text { l.e. de }(A .6)=\int d g(\operatorname{det} g \equiv 1) \exp [\operatorname{Tr}(J g)]=\text { l.d. de }(A .6) \text {. }
$$

Um exercício combinatorial [7] mostra que

$$
\left(\operatorname{det} \frac{\partial}{\partial J}\right)(\operatorname{det} J)^{i}=\frac{(i+N-1) !}{(i-1) !}(\operatorname{det} J)^{i-1} \text {. }
$$

Usando as eqs. (A.5) e (A.7) obtemos

$$
a_{i}=\frac{(i-1) !(i+N-1) !}{(i+N-1) !} a_{i-1}
$$

Com a condição inicial $a_{0}=1$ resolvemos a equação de diferença (A.8) para obtermos

$$
a_{i}=\frac{2 ! 3 ! \ldots(N-1) !}{i !(i+1) ! \ldots(i+N-1) !}
$$

que, levando em (A.5), fornece

$$
W(J)=\sum_{i=0}^{\infty} \frac{2 ! \ldots(N-1) !}{i ! \ldots(i+N-1) !}(\operatorname{det} J)^{i}
$$

Nós agora restringimos nossa análise ao grupo $S U(3)$. Vamos exemplificar o método de Creutz calculando as integrais

$$
\begin{gathered}
\mathcal{I}_{2}=\int d g g_{i_{1} j_{1}} g_{i_{2} j_{2}}^{-1}=\frac{1}{3} \delta_{i_{1} j_{2}} \delta_{j_{1} i_{2}} \\
\mathcal{I}_{3}=\int d g g_{i_{1} j_{1}} g_{i_{2} j_{2}} g_{i_{3} j_{3}}=\frac{1}{6} \epsilon_{i_{1} i_{2} i_{3}} \epsilon_{j_{1} j_{2} j_{3}} \\
\mathcal{I}_{4}=\frac{1}{8}\left(\delta_{i_{1} j_{2}} \delta_{i_{3} j_{4}} \delta_{j_{1} i_{2}} \delta_{j_{3} i_{4}}+\delta_{i_{1} j_{4}} \delta_{i_{3} j_{2}} \delta_{j_{1} i_{4}} \delta_{j_{3} i_{2}}\right) \\
-\frac{1}{24}\left(\delta_{i_{1} j_{2}} \delta_{i_{3} j_{4}} \delta_{j_{1} i_{4}} \delta_{j_{3} i_{2}}+\delta_{i_{1} j_{4}} \delta_{i_{3} j_{2}} \delta_{j_{1} i_{2}} \delta_{j_{3} i_{4}}\right) .
\end{gathered}
$$

$\operatorname{com} g, g^{-1} \in S U(3)$.

A fórmula de Creutz para calcular a integral dos elementos de calibre é dada neste caso 
por (veja (A.5) e (A.3))

$$
\sum_{r=0}^{\infty} a_{r}\left[D\left(J^{T}\right)\right]^{r}=\int d g \exp \left[\operatorname{Tr}\left(J^{T} g\right)\right]
$$

onde $\operatorname{Tr}\left(J^{T} g\right) \equiv J_{k l} g_{k l}$ e usamos $J^{T}$, ao invés de $J$, tal que no cálculo das derivadas $J$ e $g$ tenham os mesmos índices matriciais.

Os $a_{i}$ 's, neste caso, são dados explicitamente por, usando a eq. (A.9)

$$
a_{i}=\frac{2}{i !(i+1) !(i+2) !}
$$

e

$$
D(J)=\frac{1}{6} \epsilon_{k_{1} k_{2} k_{3}} \epsilon_{l_{1} l_{2} l_{3}} J_{k_{1} l_{1}} J_{k_{2} l_{2}} J_{k_{3} l_{3}} .
$$

A partir deste momento usaremos, por conveniência, a notação

$$
\partial_{k} X \equiv \frac{\partial X}{\partial J_{i_{k} j_{k}}}, \quad \partial_{k l} X \equiv \frac{\partial^{2} X}{\partial J_{i_{k} j_{k}} \partial J_{i_{l} j_{l}}}, \quad \partial_{k l^{\prime}} X \equiv \frac{\partial^{2} X}{\partial J_{i_{k} j_{k}} \partial J_{i_{l}^{\prime} j_{l}^{\prime}}}, \quad \text { etc }
$$

$\operatorname{com} X(J) \equiv X$

Vamos primeiramente determinar $\mathcal{I}_{3}$, já que, conforme veremos usaremos este resultado no cálculo de $\mathcal{I}_{2}$. Tomando a derivada do lado direito da eq. (A.13) para três campos $J$, i.e. $J_{i_{1} j_{1}}, J_{i_{2} j_{2}}$ e $J_{i_{3} j_{3}}$ e usando a notação da eq. (A.14) obtemos

$$
\left.\partial_{123}^{3} W\right|_{J=0}=\left.\partial_{123}^{3} e^{\operatorname{Tr} J^{T} g}\right|_{J=0}=\int d g g_{i_{1} j_{1}} g_{i_{2} j_{2}} g_{i_{3} j_{3}}=\mathcal{I}_{3}
$$

Por outro lado, derivando o lado esquerdo de (A.13) chegamos a

$$
\begin{aligned}
\left.\partial_{123}^{3}\left(\sum_{r=0}^{\infty} a_{r} D^{r}\right)\right|_{J=0}=\left.a_{1} \partial_{123}^{3} D\right|_{J=0}= & \frac{1}{36} \epsilon_{k_{1} k_{2} k_{3}} \epsilon_{l_{1} l_{2} l_{3}} \frac{\partial^{2}}{\partial J_{i_{1} j_{1}} \partial J_{i_{2} j_{2}}}\left(\delta_{k_{1} l_{1}, i_{3} j_{3}} J_{k_{2} l_{2}} J_{k_{3} l_{3}}\right. \\
& \left.+\delta_{k_{2} l_{2}, i_{3} j_{3}} J_{k_{1} l_{1}} J_{k_{3} \ell_{3}}+\delta_{k_{3} l_{3}, i_{3} j_{3}} J_{k_{1} l_{1}} J_{k_{2} \ell_{2}}\right)\left.\right|_{J=0} \\
= & \left.\frac{1}{12} \frac{\partial^{2}}{\partial J_{i_{1} j_{1}} \partial J_{i_{2} j_{2}}} \epsilon_{k_{1} k_{2} i_{3}} \epsilon_{l_{1} l_{2} j_{3}} J_{k_{1} l_{1}} J_{k_{2} l_{2}}\right|_{J=0} \\
= & \frac{1}{6} \epsilon_{i_{1} i_{2} i_{3} i_{3}} \epsilon_{j_{1} j_{2} j_{3}} .
\end{aligned}
$$

Notamos que os outros termos $\partial_{123}^{3} D^{n}(n \neq 1)$ são zero em $J=0$. Desta forma, combinando as eqs. (A.15) e (A.16) obtemos (A.11).

Para $\mathcal{I}_{2}$, observamos primeiramente que

$$
\mathcal{I}_{2}=\int d g g_{i_{1} j_{1}} g_{i_{2} j_{2}}^{-1}=\int d g g_{i_{1} j_{1}}(\operatorname{cof} g)_{i_{2} j_{2}}=\frac{1}{4} \epsilon_{j_{2} i_{2}^{\prime} i_{3}^{\prime}} \epsilon_{i_{2} j_{2}^{\prime} j_{3}^{\prime}} \int d g g_{i_{1} j_{1}} g_{i_{2}^{\prime} j_{2}^{\prime}} g_{i_{3}^{\prime} j_{3}^{\prime}} .
$$


Substituindo a eq. (A.11) em (A.17), obtemos

$$
\mathcal{I}_{2}=\frac{1}{4} \epsilon_{j_{2} i_{2}^{\prime} i_{3}^{\prime}} \epsilon_{i_{2} j_{2}^{\prime} j_{3}^{\prime}} \epsilon_{i_{1} i_{2}^{\prime} i_{3}^{\prime}} \epsilon_{j_{1} j_{2}^{\prime} j_{3}^{\prime}}
$$

Finalmente, usando a relação

$$
\epsilon_{i m n} \epsilon_{j m n}=2 \delta_{i j}
$$

chegamos ao resultado desejado para $\mathcal{I}_{2}$, ou seja, a eq. (A.10).

No caso de $\mathcal{I}_{4}$, procedemos como no cálculo de $\mathcal{I}_{2}$, lembrando que os $g^{-1}$ 's podem ser reeescritos em termos dos cofatores de $g$ [veja eq. (A.3)], obtemos

$$
\begin{aligned}
\mathcal{I}_{4} & =\int d g g_{i_{1} j_{1}} g_{i_{2} j_{2}}^{-1} g_{i_{3} j_{3}} g_{i_{4} j_{4}}^{-1} \\
& =\int d g g_{i_{1} j_{1}}(\operatorname{cof} g)_{i_{2} j_{2}} g_{i_{3} j_{3}}(\operatorname{cof} g)_{i_{4} j_{4}} \\
& =\frac{1}{4} \epsilon_{j_{2} i_{2}^{\prime} i_{3}^{\prime}} \epsilon_{i_{2} j_{2}^{\prime} j_{3}^{\prime}} \epsilon_{j_{4} i_{4}^{\prime} i_{5}^{\prime}} \epsilon_{i_{4} j_{4}^{\prime} j_{5}^{\prime}} \int d g g_{i_{1} j_{1}} g_{i_{2}^{\prime} j_{2}^{\prime}} g_{i_{3}^{\prime} j_{3}^{\prime}} g_{i_{3} j_{3}} g_{i_{4}^{\prime} j_{4}^{\prime}} g_{i_{5}^{\prime} j_{5}^{\prime}} .
\end{aligned}
$$

Usando a fórmula de Creutz da eq. (A.13) temos

$$
\begin{aligned}
\mathcal{I}_{4}= & \left.\frac{1}{4} \epsilon_{j_{2} i_{2}^{\prime} i_{3}^{\prime}} \epsilon_{i_{2} j_{2}^{\prime} j_{3}^{\prime}} \epsilon_{j_{4} i_{4}^{\prime} i_{5}^{\prime}} \epsilon_{i_{4} j_{4}^{\prime} j_{5}^{\prime}} \partial_{12^{\prime} 3^{\prime} 34^{\prime} 5^{\prime}}^{6} W(J)\right|_{J=0} \\
= & \left.\frac{1}{4} \epsilon_{j_{2} i_{2}^{\prime} i_{3}^{\prime}} \epsilon_{i_{2} j_{2}^{\prime} j_{3}^{\prime}} \epsilon_{j_{4} i_{4}^{\prime} i_{5}^{\prime}} \epsilon_{i_{4} j_{4}^{\prime} j_{5}^{\prime}} \partial_{12^{\prime} 3^{\prime} 34^{\prime} 5^{\prime}}^{6} \int d g e^{\operatorname{Tr}\left(J^{T} g\right)}\right|_{J=0} \\
= & \left.\frac{1}{4} \epsilon_{j_{2} i_{2}^{\prime} i_{3}^{\prime}} \epsilon_{i_{2} j_{2}^{\prime} j_{3}^{\prime}} \epsilon_{j_{4} i_{4}^{\prime} i_{5}^{\prime}} \epsilon_{i_{4} j_{4}^{\prime} j_{5}^{\prime}} \partial_{12^{\prime} 3^{\prime} 34^{\prime} 5^{\prime}}^{6}\left(\sum_{r=0}^{\infty} a_{r} D^{r}\right)\right|_{J=0} \\
= & \left.\frac{a_{2}}{4} \epsilon_{j_{2} i_{2}^{\prime} i_{3}^{\prime}} \epsilon_{i_{2} j_{2}^{\prime} j_{3}^{\prime}} \epsilon_{j_{4} i_{4}^{\prime} i_{5}^{\prime}} \epsilon_{i_{4} j_{4}^{\prime} j_{5}^{\prime}} \partial_{12^{\prime} 3^{\prime} 34^{\prime} 5^{\prime}}^{6} D^{2}\right|_{J=0} \\
= & \frac{1}{2(3 !)(4 !)}\left[\left(\partial_{12^{\prime} 3^{\prime}}^{3} D\right)\left(\partial_{34^{\prime} 5^{\prime}}^{3} D\right)+\left(\partial_{12^{\prime} 3}^{3} D\right)\left(\partial_{3^{\prime} 4^{\prime} 5^{\prime}}^{3} D\right)\right. \\
& +\left(\partial_{12^{\prime} 4^{\prime}}^{3} D\right)\left(\partial_{3^{\prime} 35^{\prime}}^{3} D\right)+\left(\partial_{12^{\prime} 5^{\prime}}^{3} D\right)\left(\partial_{3^{\prime} 34}^{3} D\right)+\left(\partial_{13^{\prime} 3}^{3} D\right)\left(\partial_{2^{\prime} 4^{\prime} 5^{\prime}}^{3} D\right) \\
& +\left(\partial_{13^{\prime} 4^{\prime}}^{3} D\right)\left(\partial_{2^{\prime} 35^{\prime}}^{3} D\right)+\left(\partial_{13^{\prime} 5^{\prime}}^{3} D\right)\left(\partial_{2^{\prime} 34^{\prime}}^{3} D\right)+\left(\partial_{134^{\prime}}^{3} D\right)\left(\partial_{2^{\prime} 3^{\prime} 5^{\prime}}^{3} D\right) \\
& \left.+\left(\partial_{135^{\prime}}^{3} D\right)\left(\partial_{2^{\prime} 3^{\prime} 4^{\prime}}^{3} D\right)+\left(\partial_{14^{\prime} 5^{\prime}}^{3} D\right)\left(\partial_{2^{\prime} 3^{\prime} 3}^{3} D\right)\right]\left.\right|_{J=0},
\end{aligned}
$$

onde a última igualdade é obtida usando $a_{2}=1 /[(3 !)(4 !)]$.

Usando na eq. (A.19), a eq. (A.18), a relação $\epsilon_{i j m} \epsilon_{k l m}=\delta_{i k} \delta_{j l}-\delta_{i l} \delta_{j k}$, obtemos para $\mathcal{I}_{4}$, reagrupando os termos, o resultado desejado. 


\section{Apêndice B}

\section{Método de desacoplamento de hiperplanos na rede}

Neste apêndice, conforme discutimos no Capítulo 2, apresentamos cálculos detalhados a respeito do método de desacoplamento de hiperplanos. Este método serve de guia indicando a combinação mais adequada dos campos simples para que tenhamos as propriedades desejadas para o decaimento da inversa convolutiva de $G, \Gamma$ e do núcleo de B-S, $K$. Através das derivadas do método de desacoplamento de hiperplanos obtemos a chamada "estrutura do produto" para derivadas. Isto é, considerando a função geral $\langle F(x) ; H(y)\rangle_{T}$, e efetuando a derivada na variável $\kappa_{p}$ (sem explicitarmos a ordem da derivada), obtemos

$$
\frac{\partial^{r}}{\partial \kappa_{p}^{r}}\langle F(x) ; H(y)\rangle_{T}=c \sum_{z}\langle F(x) ; M(z)\rangle_{T}\langle L(z) ; H(y)\rangle_{T}
$$

onde $c$ é uma constante. A "estrutura do produto" segue fixando os campos externos $F$ e $H$ de tal forma que tenhamos para a derivada de $\langle F(x) ; H(y)\rangle_{T}$ o resultado

$$
\frac{\partial^{r}}{\partial \kappa_{p}^{r}}\langle L(x) ; M(y)\rangle_{T}=c \sum_{z}\langle L(x) ; M(z)\rangle_{T}\langle L(z) ; M(y)\rangle_{T}
$$

neste caso, $F=L$ e $H=M$.

Nós agora apresentamos o cálculo das derivadas da função de dois pontos e quatro pontos para mésons. Neste apêndice, discutimos explicitamente o método de desacoplamento de hiperplanos para a direção temporal. As direções espaciais são tratadas similarmente. Os argumentos são tratados para funções de correlação em um volume finito $\Lambda \in \mathbb{Z}_{o}^{d+1}$, com cotas uniformes no volume $|\Lambda|$. A partir daí, estendidos para o volume infinito usando a consequência padrão da expansão em polímeros (veja, por exemplo, [36, 23]). Para obtermos o decaimento temporal de $\langle F(x) ; H(y)\rangle_{T}$ introduzimos a representação com variáveis duplicadas, que significa substituir a função truncada $\langle F(x) ; H(y)\rangle_{T}=\langle F(x) H(y)\rangle-\langle F(x)\rangle\langle H(y)\rangle$ pela 
expressão equivalente dependendo das variáveis independentes $\psi$ e $\bar{\psi}^{\prime}$ para obtermos

$$
\begin{aligned}
\langle F(x) ; H(y)\rangle_{T}= & \frac{1}{2 Z^{2}} \int\left[F(x)-F^{\prime}(x)\right]\left[H(y)-H^{\prime}(y)\right] e^{\left[-\mathcal{S}(\psi, \bar{\psi}, g)-\mathcal{S}\left(\psi^{\prime}, \bar{\psi}^{\prime}, g^{\prime}\right)\right]} \\
& \times d \psi d \bar{\psi} d \mu(g) d \psi^{\prime} d \bar{\psi}^{\prime} d \mu\left(g^{\prime}\right) .
\end{aligned}
$$

Lembrando da Seção 2.2, em termos da ação conectando os pontos $x^{0}=p$ e $x^{0}=p+1$, o parâmetro de "hopping" $\kappa$ é substituído pelo parâmetro complexo $\kappa_{p}$. Para $\left(x^{0} \leq p<y^{0}\right)$, temos

$$
\begin{aligned}
\langle F(x) ; H(y)\rangle_{T}= & \frac{1}{2 Z^{2}} \int\left[F(x)-F^{\prime}(x)\right]\left[H(y)-H^{\prime}(y)\right] \exp \left\{-\sum_{w^{0}=p} \kappa_{p}[A(\psi, \bar{\psi}, g, w)\right. \\
& \left.\left.+A\left(\psi^{\prime}, \bar{\psi}^{\prime}, g^{\prime}, w\right)\right]\right\} e^{\left[-\mathcal{S}(\psi, \bar{\psi}, g)-\mathcal{S}\left(\psi^{\prime}, \bar{\psi}^{\prime}, g^{\prime}\right)\right]} d \psi d \bar{\psi} d \mu(g) d \psi^{\prime} d \bar{\psi}^{\prime} d \mu\left(g^{\prime}\right) \\
\equiv & \frac{N}{2 D}
\end{aligned}
$$

onde

$$
\begin{aligned}
A(\psi, \bar{\psi}, g, w) \equiv & \frac{1}{2}\left[\bar{\psi}_{\alpha, a, f}(w) \Gamma_{\alpha \beta}^{e^{0}}\left(g_{w, w+e^{0}}\right)_{a b} \psi_{\beta, b, f}\left(w+e^{0}\right)\right. \\
& \left.+\bar{\psi}_{\alpha, a, f}\left(w+e^{0}\right) \Gamma_{\alpha \beta}^{-e^{0}}\left(g_{w+e^{0}, w}\right)_{a b} \psi_{\beta, b, f}(w)\right]
\end{aligned}
$$

e $Z^{2}$ é o fator de normalização dependendo de $\kappa_{p}$. $F^{\prime}$ e $H^{\prime}$ são funções das variáveis duplicadas $\psi^{\prime}, \bar{\psi}^{\prime}$ e $g^{\prime} . \mathcal{S}(\psi, \bar{\psi}, g)$ é a ação para os elos remanescentes, i.e., além daqueles associados com a expansão do parâmetro $\kappa_{p}$. A seguir, expandimos o numerador $N \equiv \sum_{j=0}^{\infty} N^{(j)} \kappa_{p}^{j}$ e denominador $D \equiv \sum_{j=0}^{\infty} D^{(j)} \kappa_{p}^{j}$ da eq. (B.4) em potências de $\kappa_{p}$. Para o denominador, o coeficiente de $\kappa_{p}$ é uma soma de elos. Cada termo é o produto de médias contendo um único $\psi$ ou $\bar{\psi}$ cuja integral de Fermi dá zero. O coeficiente de $\kappa_{p}^{2}$ é uma soma de termos com dois elos que devem ser coincidentes e com orientação oposta para resultar uma contribuição nãonula. Para o numerador, consideramos os coeficientes de $\kappa_{p}^{n}$. $\kappa_{p}^{0}$ é trivialmente zero. Para os coeficientes $\kappa_{p}^{1}$ e $\kappa_{p}^{3}$ a média fatoriza e cada fator tem um número ímpar de férmions resultando em zero. A integral sobre campos de calibre entre hiperplanos pode também ser usada para mostrar que a contribuição para a ordem $\kappa_{p}^{1}$ é zero. Desta forma, obtemos

$$
\begin{aligned}
\langle F(x) ; H(y)\rangle_{T}= & \frac{N}{2 D}=\frac{1}{2} \frac{N^{(2)} \kappa_{p}^{2}+N^{(4)} \kappa_{p}^{4}}{D^{(0)}+D^{(2)} \kappa_{p}^{2}} \\
= & \frac{1}{2} \frac{N^{(2)} \kappa_{p}^{2}+N^{(4)} \kappa_{p}^{4}}{D^{(0)}}-\frac{1}{2} \frac{N^{(2)} D^{(2)} \kappa_{p}^{4}}{\left[D^{(0)}\right]^{2}} \\
& +\left(\text { termos de ordem superior em } \kappa_{p}\right)
\end{aligned}
$$


Agora, mostraremos explicitamente, para $x^{0} \leq \kappa_{p}<y^{0}$, que

$$
\begin{aligned}
\langle F(x) ; H(y)\rangle_{T}= & \kappa_{p}^{2} \sum_{w^{0}=p}\left\langle F ; \Pi_{k}\right\rangle_{T}(x, w)\left\langle\mu_{k} ; H\right\rangle_{T}\left(w+e^{0}, y\right) \\
& +\kappa_{p}{ }^{4}\left\{\sum_{\vec{z} \neq \vec{w} \mid w^{0}=p} \frac{1}{2}\left\langle F ; \Pi_{k} \Pi_{l}(\vec{z})\right\rangle_{T}(x, w)\left\langle\mu_{k} \mu_{l}(\vec{z}) ; H\right\rangle_{T}\left(w+e^{0}, y\right)\right. \\
& +\sum_{w^{0}=p} \frac{9}{16}\left\langle F ; \bar{h}_{12} \bar{h}_{34}(0)\right\rangle_{T}(x, w)\left\langle h_{12} h_{34}(0) ; H\right\rangle_{T}\left(w+e^{0}, y\right) \\
& \left.+\sum_{w^{0}=p} \frac{3}{16}\left\langle F ; \bar{h}_{12} \bar{h}_{34}(0)\right\rangle_{T}(x, w)\left\langle h_{14} h_{32}(0) ; H\right\rangle_{T}\left(w+e^{0}, y\right)\right\} \\
& +\left(\text { termos de ordem superior em } \kappa_{p}\right)
\end{aligned}
$$

onde $h_{i j}=\psi_{-, a, f_{i}} \bar{\psi}_{+, a, f_{j}} / \sqrt{3}, \bar{h}_{i j}=\bar{\psi}_{-, a, f_{i}} \psi_{+, a, f_{j}} / \sqrt{3},\left\langle F ; \Pi_{k} \Pi_{l}(\vec{z})\right\rangle_{T}(x, w)=\left\langle F(x) \Pi_{k}(w) \Pi_{l}(w+\right.$ $\vec{z})\rangle-\langle F(x)\rangle\left\langle\Pi_{k}(w) \Pi_{l}(w+\vec{z})\right\rangle$, e fica subentendido que o l.d. da eq. (B.6) é avaliado em $\kappa_{p}=0$. Podemos verificar que quando $x^{0}>\kappa_{p} \geq y^{0}$ a sequinte expressão é válida

$$
\begin{aligned}
\langle F(x) ; H(y)\rangle_{T}^{*}= & \kappa_{p}^{2} \sum_{w^{0}=p}\left\langle F ; \mu_{k}\right\rangle_{T}^{*}(x, w)\left\langle\Pi_{k} ; H\right\rangle_{T}^{*}\left(w+e^{0}, y\right) \\
& +\kappa_{p}^{4}\left\{\sum_{\vec{z} \neq \vec{w} \mid w^{0}=p} \frac{1}{2}\left\langle F ; \mu_{k} \mu_{l}(\vec{z})\right\rangle_{T}^{*}(x, w)\left\langle\Pi_{k} \Pi_{l}(\vec{z}) ; H\right\rangle_{T}^{*}\left(w+e^{0}, y\right)\right. \\
& +\sum_{w^{0}=p} \frac{9}{16}\left\langle F ; h_{12} h_{34}(0)\right\rangle_{T}^{*}(x, w)\left\langle\bar{h}_{12} \bar{h}_{34}(0) ; H\right\rangle_{T}^{*}\left(w+e^{0}, y\right) \\
& \left.+\sum_{w^{0}=p} \frac{3}{16}\left\langle F ; h_{12} h_{34}(0)\right\rangle_{T}^{*}(x, w)\left\langle\bar{h}_{14} \bar{h}_{32}(0) ; H\right\rangle_{T}^{*}\left(w+e^{0}, y\right)\right\} \\
& +\left(\text { termos de ordem superior em } \kappa_{p}\right) .
\end{aligned}
$$

Observação 9 Conforme discutimos na Seção 2.2, quando $x^{0}>\kappa_{p} \geq y^{0}$ devemos adaptar o método de desacoplamento de tal forma que possamos considerar a conjugação complexa que aparece na definição da função de dois pontos dada pela Proposição 1. Desta forma, para $x^{0}>\kappa_{p} \geq y^{0}$, tomamos primeiramente o conjugado complexo da expressão para $\langle F H\rangle$, $\langle F H\rangle^{*}=\left(1 / \mathcal{N}^{*}\right) \int F H e^{-S^{*}} d \psi d \bar{\psi} d g$, onde

$S^{*} \equiv S^{*}(\psi, \bar{\psi}, g)=\frac{\kappa}{2} \sum \bar{\psi}_{\alpha, a, f}(u)\left(\Gamma_{\alpha \beta}^{\epsilon e^{\rho}}\right)^{*}\left(g_{u, u+\epsilon e^{\rho}}^{*}\right)_{a b} \psi_{\beta, b, f}\left(u+\epsilon e^{\rho}\right)+\sum_{u \in \mathbb{Z}_{o}^{d+1}} \bar{\psi}_{\alpha, a, f}(u) M_{\alpha \beta} \psi_{\beta, a, f}(u)$ lembrando que $\kappa$ é real. Agora, podemos aplicar o método de desacoplamento de hiperplanos, fazendo a extensão analítica de $\kappa$ real para o parâmetro complexo $\kappa_{p}$. Detalhes explícitos serão dados adiante.

Observamos que as médias com um único campo $\mu$ ou $\Pi$ são zero, usando a simetria de paridade do Teorema 1.

Agora mostramos o cálculo para obter a eq. (B.6). Para o coeficiente do termo com 
potência $\kappa_{p}^{2}$ temos a seguinte expressão, expandindo $S$

$$
\begin{aligned}
\frac{N^{(2)}}{2 D^{(0)}}= & \frac{1}{2 !}\left(\frac{1}{2}\right)^{2}\left(\frac{1}{2 D^{(0)}}\right) \int\left(F-F^{\prime}\right)\left(H-H^{\prime}\right)\left[2 \sum_{\vec{w}}\left(A^{+} A^{-}\right)\right] \\
& \times e^{-\mathcal{S}-\mathcal{S}^{\prime}} d \psi d \bar{\psi} d \mu(g) d \psi^{\prime} d \bar{\psi}^{\prime} d \mu\left(g^{\prime}\right)
\end{aligned}
$$

de onde obtemos

$$
\begin{aligned}
\frac{N^{(2)}}{2 D^{(0)}}= & \frac{1}{2 !}\left(\frac{1}{2}\right)^{2} \sum_{\vec{w}}\left\langle\left[F(x)-F^{\prime}(x)\right] \bar{\psi}_{\alpha_{1}, a_{1}, f_{1}} \psi_{\beta_{2}, a_{2}, f_{2}}(w)\right\rangle \\
& \times\left\langle\psi_{\beta_{1}, a_{1}, f_{1}} \bar{\psi}_{\alpha_{2}, a_{2}, f_{2}}\left(w+e^{0}\right)\left[H(y)-H^{\prime}(y)\right]\right\rangle \\
& \times \Gamma_{\alpha_{1} \beta_{1}}^{e^{0}} \Gamma_{\alpha_{2} \beta_{2}}^{-e^{0}} \int g_{a_{1} b_{1}}\left(w, w+e^{0}\right) g_{a_{2} b_{2}}\left(w+e^{0}, w\right) d \mu(g) .
\end{aligned}
$$

Usando a integral de calibre para dois campos dada pela eq. (A.10) obtemos

$$
\begin{aligned}
\frac{N^{(2)}}{2 D^{(0)}}= & \frac{1}{2 !}\left(\frac{1}{2}\right)^{2} \frac{1}{3} \sum_{\vec{w}}\left\langle\left[F(x)-F^{\prime}(x)\right] \bar{\psi}_{\alpha_{1}, a_{1}, f_{1}} \psi_{\beta_{2}, a_{1}, f_{2}}(w)\right\rangle \\
& \times\left\langle\psi_{\beta_{1}, b_{1}, f_{1}} \bar{\psi}_{\alpha_{2}, b_{1}, f_{2}}\left(w+e^{0}\right)\left[H(y)-H^{\prime}(y)\right]\right\rangle \Gamma_{\alpha_{1} \beta_{1}}^{e^{0}} \Gamma_{\alpha_{2} \beta_{2}}^{-e^{0}}
\end{aligned}
$$

Agora, nos restringimos aos casos:

(i) Para $(3+1)$-dimensões e um sabor. Neste caso os $\Gamma$ 's são dados pela equação (1.3) e omitimos os índices de isospin por tratarmos de um único sabor. Desta forma, obtemos

$$
\begin{aligned}
\frac{N^{(2)}}{2 D^{(0)}}= & \frac{1}{2 !}\left(\frac{1}{2}\right)^{2} \frac{1}{3} \sum_{\vec{w}}\left\langle\left[F(x)-F^{\prime}(x)\right] \bar{\psi}_{\alpha_{1}, a_{1}} \psi_{\beta_{2}, a_{1}}(w)\right\rangle\left\langle\psi_{\beta_{1}, b_{1}} \bar{\psi}_{\alpha_{2}, b_{1}}\left(w+e^{0}\right)\left[H(x)-H^{\prime}(x)\right]\right\rangle \\
& \times(-2) \delta_{\alpha_{1} \beta_{1}}\left(\delta_{\alpha_{1} 3}+\delta_{\alpha_{1} 4}\right)(-2) \delta_{\alpha_{2} \beta_{2}}\left(\delta_{\alpha_{1} 1}+\delta_{\alpha_{1} 2}\right) \\
= & \frac{1}{2 !} \frac{1}{3} \sum_{\vec{w}}\left\langle\left[F(x)-F^{\prime}(x)\right] \bar{\psi}_{\ell, a_{1}} \psi_{u, a_{1}}(w)\right\rangle\left\langle\psi_{\ell, b_{1}} \bar{\psi}_{u, b_{1}}\left(w+e^{0}\right)\left[H(y)-H^{\prime}(y)\right]\right\rangle .
\end{aligned}
$$

Fazendo a identificação $(1 / \sqrt{3}) \bar{\psi}_{\ell, a_{1}} \psi_{u, a_{1}} \rightarrow \bar{M}_{i}$ e $(1 / \sqrt{3}) \psi_{\ell, b_{1}} \bar{\psi}_{u, b_{1}} \rightarrow M_{i}$ de tal forma que $(\ell, u)=(3,1) \rightarrow i=1,(\ell, u)=(4,1) \rightarrow i=2,(\ell, u)=(3,2) \rightarrow i=3$ e $(\ell, u)=(4,2) \rightarrow i=4$. A seguir, inserimos entre as médias a matriz $4 \times 4$ ortogonal $A=A^{-1}=A^{T}$

$$
A=\left(\begin{array}{rrrr}
1 / \sqrt{2} & 0 & 0 & 1 / \sqrt{2} \\
0 & 1 & 0 & 0 \\
0 & 0 & 1 & 0 \\
1 / \sqrt{2} & 0 & 0 & -1 / \sqrt{2}
\end{array}\right)
$$


que age nos $M_{i}$ como $M_{i} \rightarrow A_{i j} M_{j}$ e $\bar{M}_{i} \rightarrow \Pi_{j} A_{j i}^{t}$. Desta forma obtemos

$$
\frac{N^{(2)}}{2 D^{(0)}}=\frac{1}{2 !} \sum_{\vec{w}}\left\langle\left[F(x)-F^{\prime}(x)\right] \Pi_{j}(w)\right\rangle\left\langle\mu_{j}\left(w+e^{0}\right)\left[H(y)-H^{\prime}(y)\right]\right\rangle .
$$

Fazendo $F(x)=\mu_{i}(x)$ e $H(y)=\Pi_{k}(y)$, dados pelas eqs. (2.4) e (2.5), respectivamente, obtemos a "estrutura do produto"

$$
\frac{N^{(2)}}{2 D^{(0)}}=\frac{1}{2 !} \sum_{\vec{w}}\left\langle\left[\mu_{k}(x)-\mu_{k}^{\prime}(x)\right] \Pi_{j}(w)\right\rangle\left\langle\mu_{j}\left(w+e^{0}\right)\left[\Pi_{k}(x)-\Pi_{k}^{\prime}(x)\right]\right\rangle .
$$

Por "estrutura do produto", entendemos a fixação dos "campos externos" $F$ e $H$ de tal forma que o coeficiente de $\kappa_{p}^{2}$ seja o produto de funções de dois pontos. Observando que

$$
\left\langle\left[\mu_{k}(x)-\mu_{k}^{\prime}(x)\right] \Pi_{j}(w)\right\rangle=\left\langle\mu_{k}(x) \Pi_{j}(w)\right\rangle-\left\langle\mu_{k}(x)\right\rangle\left\langle\Pi_{j}(w)\right\rangle=\left\langle\mu_{k}(x) \Pi_{j}(w)\right\rangle
$$

podemos simplificar ainda mais o lado direito de (B.14) usando a simetria de paridade do Teorema 1 e invariância por translação das funções de correlação. Explicitamente temos

$$
\left\langle\mu_{k}(x)\right\rangle=\left\langle\bar{\psi}_{\ell, a_{1}} \psi_{u, a_{1}}(x)\right\rangle=\left\langle P \bar{\psi}_{\ell, a_{1}} P \psi_{u, a_{1}}\left(x^{0},-\vec{x}\right)\right\rangle=\left\langle(-1) \bar{\psi}_{\ell, a_{1}} \psi_{u, a_{1}}(x)\right\rangle
$$

de onde concluímos que $\left\langle\mu_{k}(x)\right\rangle=0$. De forma similar, $\left\langle\Pi_{k}(x)\right\rangle=0$.

No caso em que $x^{0} \geq \kappa_{p}>y^{0}$, obtemos para a eq. (B.10)

$$
\begin{aligned}
\frac{N^{(2)}}{2 D^{(0)}}= & \frac{1}{2 !}\left(\frac{1}{2}\right)^{2} \frac{1}{3} \sum_{\vec{w}}\left\langle\left[F(x)-F^{\prime}(x)\right] \psi_{\beta_{1}, b_{1}, f_{1}} \bar{\psi}_{\alpha_{2}, b_{1}, f_{2}}\left(w+e^{0}\right)\right\rangle^{*} \\
& \times\left\langle\bar{\psi}_{\alpha_{1}, a_{1}, f_{1}} \psi_{\beta_{2}, a_{1}, f_{2}}(w)\left[H(y)-H^{\prime}(y)\right]\right\rangle^{*}\left(\Gamma_{\alpha_{1} \beta_{1}}^{e^{0}}\right)^{*}\left(\Gamma_{\alpha_{2} \beta_{2}}^{-e^{0}}\right)^{*}
\end{aligned}
$$

já usando o fato que a integral $\mathcal{I}_{2}$ é real (veja a eq. (A.10)). Usando a estrutura explícita das matrizes $\Gamma^{e^{0}}$ e $\Gamma^{-e^{0}}$ dadas pela eq. (1.3) e observando que $\left(\Gamma^{e^{0}}\right)^{*}=\Gamma^{e^{0}}$ e o mesmo para $\Gamma^{-e^{0}}$ obtemos

$$
\begin{aligned}
\frac{N^{(2)}}{2 D^{(0)}}= & \frac{1}{2 !}\left(\frac{1}{2}\right)^{2} \frac{1}{3} \sum_{\vec{w}}\left\langle\left[F(x)-F^{\prime}(x)\right] \psi_{\ell, b_{1}} \bar{\psi}_{u, b_{1}}\left(w+e^{0}\right)\right\rangle^{*} \\
& \times\left\langle\bar{\psi}_{\ell, a_{1}} \psi_{u, a_{1}}(w)\left[H(y)-H^{\prime}(y)\right]\right\rangle^{*}
\end{aligned}
$$

e a "estrutura do produto" segue como no caso anterior.

(ii) Para (2+1)-dimensões e um sabor. Neste caso, os $\Gamma$ 's são dados pela eq. (1.4) e 
novamente omitimos os índices de isospin. Desta forma, obtemos

$$
\begin{aligned}
\frac{N^{(2)}}{2 D^{(0)}}= & \frac{1}{2 !}\left(\frac{1}{2}\right)^{2} \frac{1}{3} \sum_{\vec{w}}\left\langle\left[F(x)-F^{\prime}(x)\right] \bar{\psi}_{\alpha_{1}, a_{1}} \psi_{\beta_{2}, a_{1}}(w)\right\rangle\left\langle\psi_{\beta_{1}, b_{1}} \bar{\psi}_{\alpha_{2}, b_{1}}\left(w+e^{0}\right)\left[H(y)-H^{\prime}(y)\right]\right\rangle \\
& \times(-2) \delta_{\alpha_{1} \beta_{1}} \delta_{\alpha_{1}-}(-2) \delta_{\alpha_{2} \beta_{2}} \delta_{\alpha_{1}+} \\
= & \frac{1}{2 !} \frac{1}{3} \sum_{\vec{w}}\left\langle\left[F(x)-F^{\prime}(x)\right] \bar{\psi}_{-, a_{1}} \psi_{+, a_{1}}(w)\right\rangle\left\langle\psi_{-, b_{1}} \bar{\psi}_{+, b_{1}}\left(w+e^{0}\right)\left[H(y)-H^{\prime}(y)\right]\right\rangle .
\end{aligned}
$$

Neste caso fazemos as identificações $(1 / \sqrt{3}) \psi_{-, b_{1}} \bar{\psi}_{+, b_{1}} \rightarrow \mu \mathrm{e}(1 / \sqrt{3}) \bar{\psi}_{-, a_{1}} \psi_{+, a_{1}} \rightarrow \Pi$ para obtermos

$$
\frac{N^{(2)}}{2 D^{(0)}}=\frac{1}{2 !} \frac{1}{3} \sum_{\vec{w}}\left\langle\left[F(x)-F^{\prime}(x)\right] \Pi(w)\right\rangle\left\langle\mu\left(w+e^{0}\right)\left[H(y)-H^{\prime}(y)\right]\right\rangle .
$$

Fazendo $F(x) \equiv \mu$ e $H(y) \equiv \Pi$ e usando a simetria de paridade (conforme anteriormente mostramos que $\langle\mu(x)\rangle=0=\langle\Pi(x)\rangle)$ obtemos a "estrutura do produto"

$$
\frac{N^{(2)}}{2 D^{(0)}}=\frac{1}{2 !} \sum_{\vec{w}}\langle\mu(x) \Pi(w)\rangle\left\langle\mu\left(w+e^{0}\right) \Pi(y)\right\rangle .
$$

(iii) Para $(2+1)$-dimensões e dois sabores. Neste caso os $\Gamma^{\prime}$ 's são dados pela eq. (1.4). Desta forma obtemos

$$
\begin{aligned}
\frac{N^{(2)}}{2 D^{(0)}}= & \frac{1}{2 !}\left(\frac{1}{2}\right)^{2} \frac{1}{3} \sum_{\vec{w}}\left\langle\left[F(x)-F^{\prime}(x)\right] \bar{\psi}_{\alpha_{1}, a_{1}, f_{1}} \psi_{\beta_{2}, a_{1}, f_{2}}(w)\right\rangle \\
& \times\left\langle\psi_{\beta_{1}, b_{1}, f_{1}} \bar{\psi}_{\alpha_{2}, b_{1}, f_{2}}\left(w+e^{0}\right)\left[H(y)-H^{\prime}(y)\right]\right\rangle(-2) \delta_{\alpha_{1} \beta_{1}} \delta_{\alpha_{1}-}(-2) \delta_{\alpha_{2} \beta_{2}} \delta_{\alpha_{1}+} \\
= & \frac{1}{2 !} \frac{1}{3} \sum_{\vec{w}}\left\langle\left[F(x)-F^{\prime}(x)\right] \bar{\psi}_{-, a_{1}, f_{1}} \psi_{+, a_{1}, f_{2}}(w)\right\rangle\left\langle\psi_{-, b_{1}, f_{1}} \bar{\psi}_{+, b_{1}, f_{2}}\left(w+e^{0}\right)\left[H(y)-H^{\prime}(y)\right]\right\rangle
\end{aligned}
$$

Nós agora consideramos o coeficiente de $\kappa_{p}^{4}$ com dois termos a considerar $(1 / 2) N^{(4)} / D^{(0)}$ e $(1 / 2) N^{(2)} D^{(2)} /\left[D^{(0)}\right]^{2}$. Para o termo $(1 / 2) N^{(2)} D^{(2)} /\left[D^{(0)}\right]^{2}$ temos que

$$
\frac{N^{(2)} D^{(2)}}{2\left[D^{(0)}\right]^{2}}=\frac{N^{(2)}}{2 D^{(0)}} \frac{D^{(2)}}{D^{(0)}}
$$

e o fator $(1 / 2) N^{(2)} / D^{(0)}$ já foi calculado anteriormente. Para $D^{(2)} / D^{(0)}$ temos

$$
\frac{D^{(2)}}{D^{(0)}}=\frac{1}{3} \sum_{\vec{w}}\left\langle\bar{\psi}_{-, a_{1}, f_{1}} \psi_{+, a_{1}, f_{2}}(w)\right\rangle\left\langle\psi_{-, b_{1}, f_{1}} \bar{\psi}_{+, b_{1}, f_{2}}\left(w+e^{0}\right)\right\rangle
$$

e cada média na eq. (B.21) é zero usando a simetria de paridade. Desta forma, a eq. (B.21) 
dá uma contribuição nula.

Considerando o termo $N^{(4)} / 2 D^{(0)}$ na eq. (B.4), temos

$$
\begin{aligned}
\frac{N^{(4)}}{2 D^{(0)}}= & \frac{1}{4 !}\left(\frac{1}{2}\right)^{4}\left(\frac{1}{2 D^{(0)}}\right) \int\left(F-F^{\prime}\right)\left(H-H^{\prime}\right) \\
& \times\left[2 4 \sum _ { ( \vec { w } _ { 1 } , \vec { w } _ { 2 } ) | w _ { 1 } \neq w _ { 2 } } \left(A_{1}^{+} A_{1}^{-} A_{2}^{+} A_{2}^{-}\right.\right. \\
& \left.\left.+A_{1}^{+} A_{1}^{-} A_{2}^{\prime+} A_{2}^{\prime-}\right)+12 \sum_{\vec{w}_{1}}\left(A_{1}^{+}\right)^{2}\left(A_{1}^{-}\right)^{2}\right] \\
& \times e^{-\mathcal{S}-\mathcal{S}^{\prime}} d \psi d \bar{\psi} d \mu(g) d \psi^{\prime} d \bar{\psi}^{\prime} d \mu\left(g^{\prime}\right),
\end{aligned}
$$

onde

$$
\begin{gathered}
A_{j}^{+} \equiv \bar{\psi}_{\alpha, a, f}\left(w_{j}\right) \Gamma_{\alpha \beta}^{e^{0}}\left(g_{w_{j}, w_{j}+e^{0}}\right)_{a b} \psi_{\beta, b, f}\left(w_{j}+e^{0}\right), \\
A_{j}^{-} \equiv \bar{\psi}_{\alpha, a, f}\left(w_{j}+e^{0}\right) \Gamma_{\alpha \beta}^{-e^{0}}\left(g_{w_{j}+e^{0}, w_{j}}\right)_{a b} \psi_{\beta, b, f}\left(w_{j}\right)
\end{gathered}
$$

e $F, \mathcal{S}$ são notações abreviadas para $F(x), \mathcal{S}(\psi, \bar{\psi}, g)$, respectivamente, e similarmente para $F^{\prime}, H, H^{\prime}$ e $\mathcal{S}^{\prime}$.

Para o coeficiente de $\kappa_{p}^{4}$ consideramos somente o caso $(2+1)$-dimensões. Restringimos nossa análise para o caso de dois sabores, sendo um sabor completamente análogo. Neste caso, o primeiro termo do lado direito de (B.23) fornece

$$
\begin{aligned}
& \sum_{\vec{w}_{1}, \vec{w}_{2}}\left\langle\left[F(x)-F^{\prime}(x)\right]\left[H(y)-H^{\prime}(y)\right] A_{1}^{+} A_{1}^{-} A_{2}^{+} A_{2}^{-}\right\rangle \\
& =\frac{1}{2 !}\left(\frac{1}{2}\right)^{4} \sum_{\vec{w}}\left\langle\left[F(x)-F^{\prime}(x)\right] \bar{\psi}_{\alpha_{1}, a_{1}, f_{1}} \psi_{\beta_{2}, b_{2}, f_{2}} \bar{\psi}_{\alpha_{3}, a_{3}, f_{3}} \psi_{\beta_{4}, b_{4}, f_{4}}(w)\right\rangle \\
& \times\left\langle\psi_{\beta_{1}, b_{1}, f_{1}} \bar{\psi}_{\alpha_{2}, b_{1}, f_{2}} \psi_{\beta_{3}, b_{3}, f_{3}} \bar{\psi}_{\alpha_{4}, b_{4}, f_{4}}\left(w+e^{0}\right)\left[H(y)-H^{\prime}(y)\right]\right\rangle \\
& \times \Gamma_{\alpha_{1} \beta_{1}}^{e^{0}} \Gamma_{\alpha_{2} \beta_{2}}^{-e^{0}} \Gamma_{\alpha_{3} \beta_{3}}^{e^{0}} \Gamma_{\alpha_{4} \beta_{4}}^{-e^{0}} \int g_{a_{1} b_{1}}\left(w, w+e^{0}\right) g_{a_{2} b_{2}}\left(w+e^{0}, w\right) d \mu(g) \\
& \times \int g_{a_{3} b_{3}}\left(w, w+e^{0}\right) g_{a_{4} b_{4}}\left(w+e^{0}, w\right) d \mu(g) \\
& =\frac{1}{2 !} \frac{1}{9} \sum_{\vec{w}_{1}, \vec{w}_{2}}\left\langle\left[F(x)-F^{\prime}(x)\right] \bar{\psi}_{-, a_{1}, f_{1}} \psi_{+, a_{1}, f_{2}}\left(w^{0}, \vec{w}_{1}\right) \bar{\psi}_{-, a_{3}, f_{3}} \psi_{+, a_{3} f_{4}}\left(w^{0}, \vec{w}_{2}\right)\right\rangle \\
& \times\left\langle\psi_{-, b_{1}, f_{1}} \bar{\psi}_{+, b_{1}, f_{2}}\left(w^{0}+e^{0}, \vec{w}_{1}\right) \psi_{-, b_{3}, f_{3}} \bar{\psi}_{+, b_{3}, f_{4}}\left(w^{0}+e^{0}, \vec{w}_{2}\right)\left[H(y)-H^{\prime}(y)\right]\right\rangle .
\end{aligned}
$$

Nós agora fazemos a identificação $\left(f_{1}, f_{2}\right) \rightarrow i$ e $\left(f_{3}, f_{4}\right) \rightarrow j$, para $i, j=1, \ldots, 4$, com $(+,+) \equiv 1,(+,-) \equiv 2,(-,+) \equiv 3$ e $(-,-) \equiv 4$. A seguir, definimos os campos compostos $\bar{M}_{i} \equiv \bar{\psi}_{-, a, f_{1}} \psi_{+, a, f_{2}} / \sqrt{3}, M_{i} \equiv \psi_{-, a, f_{1}} \bar{\psi}_{+, a, f_{2}} / \sqrt{3}$, e a mudança de variáveis é representada pela matrix $4 \times 4 A$ definida na eq. (B.12), mas agindo agora no grau de liberdade de isospin, como 
$\bar{M}_{i} \rightarrow \Pi_{m} A_{m i}^{t}$ e $M_{i} \rightarrow A_{i n} \mu_{n}$. Desta forma obtemos

$$
\begin{aligned}
& \sum_{\vec{w}_{1}, \vec{w}_{2}}\left\langle\left[F(x)-F^{\prime}(x)\right]\left[H(y)-H^{\prime}(y)\right] A_{1}^{+} A_{1}^{-} A_{2}^{+} A_{2}^{-}\right\rangle \\
& =\frac{1}{2 !} \frac{1}{9} \sum_{\vec{w}_{1}, \vec{w}_{2}}\left\langle\left[F(x)-F^{\prime}(x)\right] \Pi_{k}\left(w^{0}, \vec{w}_{1}\right) \Pi_{l}\left(w^{0}, \vec{w}_{2}\right)\right\rangle \\
& \times\left\langle\mu_{k}\left(w^{0}+e^{0}, \vec{w}_{1}\right) \mu_{l}\left(w^{0}+e^{0}, \vec{w}_{2}\right)\left[H(y)-H^{\prime}(y)\right]\right\rangle .
\end{aligned}
$$

e a "estrutura do produto" segue se tomarmos $F \equiv \mu_{i} \mu_{j}$ e $H \equiv \Pi_{m} \Pi_{n}$.

Para o segundo termo de (B.23) temos

$$
\begin{aligned}
& \sum_{\vec{w}_{1}, \vec{w}_{2}}\left\langle\left[F(x)-F^{\prime}(x)\right]\left[H(y)-H^{\prime}(y)\right] A_{1}^{+} A_{1}^{-} A_{2}^{+} A_{2}^{-}\right\rangle \\
& =\frac{1}{2 !}\left(\frac{1}{2}\right)^{4} \sum_{\vec{w}}\left\langle\left[F(x)-F^{\prime}(x)\right] \bar{\psi}_{\alpha_{1}, a_{1}, f_{1}} \psi_{\beta_{2}, b_{2}, f_{2}} \bar{\psi}_{\alpha_{3}, a_{3}, f_{3}}^{\prime} \psi_{\beta_{4}, b_{4}, f_{4}}^{\prime}(w)\right\rangle \\
& \times\left\langle\psi_{\beta_{1}, b_{1}, f_{1}} \bar{\psi}_{\alpha_{2}, b_{1}, f_{2}} \psi_{\beta_{3}, b_{3}, f_{3}}^{\prime} \bar{\psi}_{\alpha_{4}, b_{4}, f_{4}}^{\prime}\left(w+e^{0}\right)\left[H(y)-H^{\prime}(y)\right]\right\rangle \\
& \times \Gamma_{\alpha_{1} \beta_{1}}^{e^{0}} \Gamma_{\alpha_{2} \beta_{2}}^{-e^{0}} \Gamma_{\alpha_{3} \beta_{3}}^{e^{0}} \Gamma_{\alpha_{4} \beta_{4}}^{-e^{0}} \int g_{a_{1} b_{1}}\left(w, w+e^{0}\right) g_{a_{2} b_{2}}\left(w+e^{0}, w\right) d \mu(g) \\
& \times \int g_{a_{3} b_{3}}\left(w, w+e^{0}\right) g_{a_{4} b_{4}}\left(w+e^{0}, w\right) d \mu(g) \\
& =\frac{1}{2 !} \frac{1}{9} \sum_{\vec{w}_{1}, \vec{w}_{2}}\left\langle\left[F(x)-F^{\prime}(x)\right] \bar{\psi}_{-, a_{1}, f_{1}} \psi_{+, a_{1}, f_{2}}\left(w^{0}, \vec{w}_{1}\right) \bar{\psi}_{-, a_{3}, f_{3}}^{\prime} \psi_{+, a_{3} f_{4}}^{\prime}\left(w^{0}, \vec{w}_{2}\right)\right\rangle \\
& \times\left\langle\psi_{-, b_{1}, f_{1}} \bar{\psi}_{+, b_{1}, f_{2}}\left(w^{0}+e^{0}, \vec{w}_{1}\right) \psi_{-, b_{3}, f_{3}}^{\prime} \bar{\psi}_{+, b_{3}, f_{4}}^{\prime}\left(w^{0}+e^{0}, \vec{w}_{2}\right)\left[H(y)-H^{\prime}(y)\right]\right\rangle .
\end{aligned}
$$

Observando que

$$
\begin{aligned}
& \left\langle\left[F(x)-F^{\prime}(x)\right] \bar{\psi}_{-, a_{1}, f_{1}} \psi_{+, a_{1}, f_{2}}\left(w^{0}, \vec{w}_{1}\right) \bar{\psi}_{-, a_{3}, f_{3}}^{\prime} \psi_{+, a_{3} f_{4}}^{\prime}\left(w^{0}, \vec{w}_{2}\right)\right\rangle \\
& =\left\langle F(x) \bar{\psi}_{-, a_{1}, f_{1}} \psi_{+, a_{1}, f_{2}}\left(w^{0}, \vec{w}_{1}\right)\right\rangle\left\langle\bar{\psi}_{-, a_{3}, f_{3}} \psi_{+, a_{3} f_{4}}\left(w^{0}, \vec{w}_{2}\right)\right\rangle \\
& \quad-\left\langle F(x) \bar{\psi}_{-, a_{3}, f_{3}} \psi_{+, a_{3} f_{4}}\left(w^{0}, \vec{w}_{2}\right)\right\rangle\left\langle\bar{\psi}_{-, a_{1}, f_{1}} \psi_{+, a_{1}, f_{2}}\left(w^{0}, \vec{w}_{1}\right)\right\rangle
\end{aligned}
$$

obtemos zero para expressão (B.26), lembrando que $\left\langle\bar{\psi}_{-, a_{1}, f_{1}} \psi_{+, a_{1}, f_{2}}\right\rangle=\left\langle\bar{\psi}_{-, a_{3}, f_{3}} \psi_{+, a_{3} f_{4}}\right\rangle=0$ usando a simetria de paridade.

Na sequência, calculamos o terceiro termo do lado direito da eq. (B.23). Em termos de 
médias, o terceiro termo na eq. (B.23) é dado por

$$
\begin{aligned}
& \frac{1}{4} \frac{6}{4 !}\left(\frac{1}{2}\right)^{4} \sum_{\vec{w}_{1}}\left\langle\left(F-F^{\prime}\right)\left(H-H^{\prime}\right)\left(A_{1}^{+}\right)^{2}\left(A_{1}^{-}\right)^{2}\right\rangle= \\
& \frac{1}{4} \frac{6}{4 !}\left(\frac{1}{2}\right)^{4} \sum_{\vec{w}_{1}}\left\langle\left(F-F^{\prime}\right) \bar{\psi}_{\alpha_{1}, a_{1}, f_{1}} \psi_{\beta_{2}, b_{2}, f_{2}} \bar{\psi}_{\alpha_{3}, a_{3}, f_{3}} \psi_{\beta_{4}, b_{4}, f_{4}}\left(p, \vec{w}_{1}\right)\right\rangle \\
& \times\left\langle\psi_{\beta_{1}, b_{1} f_{1}} \bar{\psi}_{\alpha_{2}, a_{2}, f_{2}} \psi_{\beta_{3}, b_{3}, f_{3}} \bar{\psi}_{\alpha_{4}, a_{4}, f_{4}}\left(p+1, \vec{w}_{1}\right)\left(H-H^{\prime}\right)\right\rangle \\
& \times \Gamma_{\alpha_{1} \beta_{1}}^{e^{0}} \Gamma_{\alpha_{2} \beta_{2}}^{-e^{0}} \Gamma_{\alpha_{3} \beta_{3}}^{e^{0}} \Gamma_{\alpha_{4} \beta_{4}}^{-e^{0}} \int g_{a_{1} b_{1}} g_{a_{2} b_{2}}^{-1} g_{a_{3} b_{3}} g_{a_{4} b_{4}}^{-1} d \mu(g)= \\
& \frac{1}{16} \sum_{\vec{w}_{1}}\left\langle\left(F-F^{\prime}\right) \bar{\psi}_{-, a_{1}, f_{1}} \psi_{+, a_{1}, f_{2}} \bar{\psi}_{-, a_{3}, f_{3}} \psi_{+, a_{3}, f_{4}}\left(p, \vec{w}_{1}\right)\right\rangle \\
& \times\left\langle\psi_{-, b_{1}, f_{1}} \bar{\psi}_{+, b_{1}, f_{2}} \psi_{-, b_{3}, f_{3}} \bar{\psi}_{+, b_{3} f_{4}}\left(p+1, \vec{w}_{1}\right)\left(H-H^{\prime}\right)\right\rangle \\
& +\frac{1}{48} \sum_{\vec{w}_{1}}\left\langle\left(F-F^{\prime}\right) \bar{\psi}_{-, a_{1}, f_{1}} \psi_{+, a_{1}, f_{2}} \bar{\psi}_{-, a_{3}, f_{3}} \psi_{+, a_{3}, f_{4}}\left(p, \vec{w}_{1}\right)\right\rangle \\
& \times\left\langle\psi_{-, b_{1}, f_{1}} \bar{\psi}_{+, b_{1}, f_{4}} \psi_{-, a_{2}, f_{3}} \bar{\psi}_{+, a_{2}, f_{2}}\left(p+1, \vec{w}_{1}\right)\left(H-H^{\prime}\right)\right.
\end{aligned}
$$

Na última igualdade, usamos a expressão para a integral de calibre $\mathcal{I}_{4}$, dada pela eq. (A.12), e a estrutura matricial de $\Gamma^{\epsilon e^{0}}(\epsilon= \pm 1)$.

Agora, conforme fizemos anteriormente, fazemos a identificação $\left(f_{1}, f_{2}\right) \rightarrow i$ e $\left(f_{3}, f_{4}\right) \rightarrow$ $j$, para $i, j=1, \ldots, 4$, com $(+,+) \equiv 1,(+,-) \equiv 2,(-,+) \equiv 3$ e $(-,-) \equiv 4$, definimos os campos $\bar{M}_{i} \equiv \bar{\psi}_{-, a, f_{1}} \psi_{+, a, f_{2}} / \sqrt{3}, M_{i} \equiv \psi_{-, a, f_{1}} \bar{\psi}_{+, a, f_{2}} / \sqrt{3}$, e introduzimos a mudança de variáveis representada pela matrix $A$ da eq. (B.12). Desta forma obtemos, para eq. (B.27),

$$
\begin{aligned}
& \frac{9}{16} \sum_{\vec{w}_{1}}\left\langle\left(F-F^{\prime}\right) \Pi_{i} \Pi_{j}\left(p, \vec{w}_{1}\right)\right\rangle\left\langle\mu_{i} \mu_{j}\left(p+1, \vec{w}_{1}\right)\left(H-H^{\prime}\right)\right\rangle \\
& +\frac{3}{16} \sum_{\vec{w}_{1}}\left\langle\left(F-F^{\prime}\right) \Pi_{i} \Pi_{j}\left(p, \vec{w}_{1}\right)\right\rangle\left\langle L_{i j}\left(p+1, \vec{w}_{1}\right)\left(H-H^{\prime}\right)\right\rangle \\
& =\frac{9}{16} \sum_{\vec{w}_{1}}\left[\left\langle F ; \Pi_{i} \Pi_{j}\right\rangle\left(x,\left(p, \vec{w}_{1}\right)\right)\left\langle\mu_{i} \mu_{j}, H\right\rangle\left(\left(p+1, \vec{w}_{1}\right), y\right)\right] \\
& +\frac{3}{16} \sum_{\vec{w}_{1}}\left\langle\left(F-F^{\prime}\right) \Pi_{i} \Pi_{j}\left(p, \vec{w}_{1}\right)\right\rangle\left\langle L_{i j}\left(p+1, \vec{w}_{1}\right)\left(H-H^{\prime}\right)\right\rangle
\end{aligned}
$$

onde $L_{i j}$ é dado por combinações complexas dos campos $M$. Nós obtemos

$$
\begin{gathered}
L_{11}=(1 / 2)\left(M_{1} M_{1}+M_{2} M_{3}+M_{3} M_{2}+M_{4} M_{4}\right), L_{12}=(1 / \sqrt{2})\left(M_{2} M_{1}+M_{4} M_{2}\right), \\
L_{13}=(1 / \sqrt{2})\left(M_{1} M_{3}+M_{3} M_{4}\right), L_{14}=(1 / 2)\left(M_{1} M_{1}-M_{2} M_{3}+M_{3} M_{2}-M_{4} M_{4}\right), \\
L_{21}=(1 / \sqrt{2})\left(M_{1} M_{2}+M_{2} M_{4}\right), L_{22}=M_{2} M_{2}, L_{23}=(1 / 2) M_{1} M_{4},
\end{gathered}
$$




$$
\begin{gathered}
L_{24}=(1 / \sqrt{2})\left(M_{1} M_{2}-M_{2} M_{3}\right), L_{31}=(1 / \sqrt{2})\left(M_{3} M_{1}+M_{4} M_{3}\right), L_{32}=M_{4} M_{1}, \\
L_{33}=M_{3} M_{3}, L_{34}=(1 / \sqrt{2})\left(M_{3} M_{1}-M_{4} M_{3}\right), \\
L_{41}=(1 / 2)\left(M_{1} M_{1}+M_{2} M_{3}-M_{3} M_{2}-M_{4} M_{4}\right), L_{42}=(1 / \sqrt{2})\left(M_{2} M_{1}-M_{4} M_{2}\right), \\
L_{43}=(1 / \sqrt{2})\left(M_{1} M_{3}-M_{3} M_{4}\right) \text { e } L_{44}=(1 / 2)\left(M_{1} M_{1}-M_{2} M_{3}-M_{3} M_{2}+M_{4} M_{4}\right) .
\end{gathered}
$$

Um ponto importante discutido no capítulo três é o efeito da troca da integral $\mathcal{I}_{4}$ pelo produto de duas integrais de calibre de dois campos, i.e., $\mathcal{I}_{2}$. Para obtermos o efeito desta troca substituímos $\mathcal{I}_{4}$ por $\mathcal{I}_{2}^{2}$ na eq. (B.27) para obtermos

$$
\begin{aligned}
& \frac{1}{4} \frac{6}{4 !}\left(\frac{1}{2}\right)^{4} \sum_{\vec{w}_{1}}\left\langle\left(F-F^{\prime}\right)\left(H-H^{\prime}\right)\left(A_{1}^{+}\right)^{2}\left(A_{1}^{-}\right)^{2}\right\rangle= \\
& \frac{1}{4} \frac{6}{4 !}\left(\frac{1}{2}\right)^{4} \sum_{\vec{w}_{1}}\left\langle\left(F-F^{\prime}\right) \bar{\psi}_{\alpha_{1}, a_{1}, f_{1}} \psi_{\beta_{2}, b_{2}, f_{2}} \bar{\psi}_{\alpha_{3}, a_{3}, f_{3}} \psi_{\beta_{4}, b_{4}, f_{4}}\left(p, \vec{w}_{1}\right)\right\rangle \\
& \times\left\langle\psi_{\beta_{1}, b_{1} f_{1}} \bar{\psi}_{\alpha_{2}, a_{2}, f_{2}} \psi_{\beta_{3}, b_{3}, f_{3}} \bar{\psi}_{\alpha_{4}, a_{4}, f_{4}}\left(p+1, \vec{w}_{1}\right)\left(H-H^{\prime}\right)\right\rangle \\
& \times \Gamma_{\alpha_{1} \beta_{1}}^{e^{0}} \Gamma_{\alpha_{2} \beta_{2}}^{-e^{0}} \Gamma_{\alpha_{3} \beta_{3}}^{e^{0}} \Gamma_{\alpha_{4} \beta_{4}}^{-e^{0}} \int g_{a_{1} b_{1}} g_{a_{2} b_{2}}^{-1} \int g_{a_{3} b_{3}} g_{a_{4} b_{4}}^{-1} d \mu(g) \\
& =\frac{3}{4} \sum_{\vec{w}_{1}}\left\langle\left(F-F^{\prime}\right) \Pi_{i} \Pi_{j}\left(p, \vec{w}_{1}\right)\right\rangle\left\langle\mu_{i} \mu_{j}\left(p+1, \vec{w}_{1}\right)\left(H-H^{\prime}\right)\right\rangle
\end{aligned}
$$

De agora em diante, restringimos nossa atenção para o setor de isospin total zero. Tomando as duas combinações que fornecem isospin zero para $F$ e $H$, o termo $\kappa_{p}^{2}$ se torna,

$$
\sum_{\vec{w}}\left\langle\left[\mathcal{T}_{i}^{\mu}\left(x_{1} x_{2}\right)\right]^{\prime} \Pi_{k}(p, \vec{w})\right\rangle\left\langle\Pi_{k}(p+1, \vec{w})\left[\mathcal{T}_{j}^{\Pi}\left(x_{3} x_{4}\right)\right]^{\prime}\right\rangle \kappa_{p}^{2}
$$

$\operatorname{com}\left[\mathcal{T}_{j}^{\Pi}\left(x_{3} x_{4}\right)\right]^{\prime}=\mathcal{T}_{j}^{\Pi}\left(x_{3} x_{4}\right)-\left\langle\mathcal{T}_{j}^{\Pi}\left(x_{3} x_{4}\right)\right\rangle$. Pelas relações de ortogonalidade para o isospin total, somente o termo $k=1$ contribui, e cada fator \langle\rangle é separadamente zero pela simetria de paridade. Podemos mostrar que o termo resultante, para $k=1$, também é zero, desta vez, usando conjugação de carga.

Nós agora consideramos o termo de ordem $\kappa_{p}^{4}$. Nós notamos que se $\Pi_{k} \Pi_{l}$ ou $\mu_{k} \mu_{l}$ são substituídos por novos campos obtidos por uma transformação ortogonal agindo nos campos originais, então a mesma estrutura de derivada vale para os novos campos. Desta forma, primeiro transformamos para os campos $\Pi_{00}=\Pi_{1}, \Pi_{11}=-\Pi_{2}, \Pi_{1-1}=\Pi_{3}, \Pi_{10}=\Pi_{4}$, notando que o produto tensorial da transformação é ortogonal. A seguir, introduzimos os campos $\mathcal{T}_{i}^{\Pi}$, relacionados com os campos $\Pi_{I_{1} m_{1}} \Pi_{I_{2} m_{2}}$ pelos coeficientes de C-G. Estes C-G 
estão novamente associados a uma transformação ortogonal. Finalmente, obtemos

$$
\begin{aligned}
& \frac{9}{16} \sum_{\vec{w}_{1}}\left\langle\left[\mathcal{T}_{i}^{\mu}\right]^{\prime}\left[\mathcal{T}_{k}^{\Pi}\right]^{\prime}\left(p, \vec{w}_{1}\right)\right\rangle\left\langle\left[\mathcal{T}_{k}^{\mu}\right]^{\prime}\left(p+1, \vec{w}_{1}\right)\left[\mathcal{T}_{j}^{\Pi}\right]^{\prime}\right\rangle \\
& +\frac{3}{16} \sum_{\vec{w}_{1}}\left\langle\left[\mathcal{T}_{i}^{\mu}\right]^{\prime}\left[\mathcal{T}_{l}^{\Pi}\right]^{\prime}\left(p, \vec{w}_{1}\right)\right\rangle C_{l k}\left\langle\left[\mathcal{T}_{k}^{\mu}\right]^{\prime}\left(p+1, \vec{w}_{1}\right)\left[\mathcal{T}_{j}^{\Pi}\right]^{\prime}\right\rangle,
\end{aligned}
$$

com

$$
C=\left(C_{l k}\right)=\frac{1}{2}\left(\begin{array}{cc}
1 & -\sqrt{3} \\
-\sqrt{3} & -1
\end{array}\right),
$$

e notamos que $C$ pode ser reescrito em termos das projeções ortogonais $P$ e $P_{1}$ dadas, respectivamente, pelas eqs. (4.16) e (4.17), como

$$
C=-P+P_{1}
$$

Para calcularmos as derivadas de $K$ pelo método de desacoplamento de hiperplanos precisamos determinar as derivadas de $M^{-1}$ e $M_{0}^{-1}$, neste caso usaremos as relações $M M^{-1}=I$ e $M_{0} M_{0}^{-1}=I$. O procedimento consiste em derivarmos sucessivamente as relações anteriores usando os resultados obtidos neste Apêndice para a derivadas de $M$ e $M_{0}$, da mesma forma que fizemos no Lema 2.

Na sequência usamos a notação $M^{(n)}$ para designar a $n$-ésima derivada de $M$ em relação a $\kappa_{p}$ no ponto $\kappa_{p}=0$. Nós temos finalmente para a quarta derivada de $M$ o resultado, usando (B.29),

$$
\begin{aligned}
& M^{(4)}\left(x_{1}, x_{2}, x_{3}, x_{4}\right)= \\
& \frac{1}{2} \sum_{\vec{w}_{1}, \vec{w}_{2} \mid \vec{w}_{1} \neq \vec{w}_{2}} M^{(0)}\left(x_{1}, x_{2},\left(p, \vec{w}_{1}\right),\left(p, \vec{w}_{2}\right)\right) M^{(0)}\left(\left(p+1, \vec{w}_{1}\right),\left(p+1, \vec{w}_{2}\right), x_{3}, x_{4}\right) \\
& +\frac{9}{16} \sum_{\vec{w}} M^{(0)}\left(x_{1}, x_{2},(p, \vec{w}),(p, \vec{w})\right) M^{(0)}\left((p+1, \vec{w}),(p+1, \vec{w}), x_{3}, x_{4}\right) \\
& +\frac{3}{16} \sum_{\vec{w}} M^{(0)}\left(x_{1}, x_{2},(p, \vec{w}),(p, \vec{w})\right) C M^{(0)}\left((p+1, \vec{w}),(p+1, \vec{w}), x_{3}, x_{4}\right) .
\end{aligned}
$$

O segundo e terceiro termos do lado direito da eq. (B.30) podem ser combinados para chegarmos a

$$
\begin{aligned}
& M^{(4)}\left(x_{1}, x_{2}, x_{3}, x_{4}\right)= \\
& \frac{1}{2} \sum_{\vec{w}_{1}, \vec{w}_{2} \mid \vec{w}_{1} \neq \vec{w}_{2}} M^{(0)}\left(x_{1}, x_{2},\left(p, \vec{w}_{1}\right),\left(p, \vec{w}_{2}\right)\right) M^{(0)}\left(\left(p+1, \vec{w}_{1}\right),\left(p+1, \vec{w}_{2}\right), x_{3}, x_{4}\right) \\
& +\frac{1}{2} \sum_{\vec{w}} M^{(0)}\left(x_{1}, x_{2},(p, \vec{w}),(p, \vec{w})\right) \Gamma M^{(0)}\left((p+1, \vec{w}),(p+1, \vec{w}), x_{3}, x_{4}\right) .
\end{aligned}
$$

onde $\Gamma$ é a matriz agindo no grau de liberdade de isospin, dada por

$$
\Gamma=\frac{3}{4} P+\frac{3}{2} P_{1}
$$


Agora, nós explicamos como a estrutura do produto do lado direito da eq. (B.31) levará ao decaimento adequado para o núcleo de B-S, K. Para isto, reescrevemos a eq. (B.31) esquematicamente como

$$
M^{(4)}=\frac{1}{2} M^{(0)} \circledast M^{(0)}+\frac{1}{2} M^{(0)} \odot \Gamma M^{(0)},
$$

onde os produtos $\circledast$ e $\odot$ levam em conta as restrições $\vec{w}_{1} \neq \vec{w}_{2}$ e $\vec{w}_{1}=\vec{w}_{2}=\vec{w}$, respectivamente. O segundo termo na eq. (B.32) vem da contribuição de quatro campos sobrepostos (dois com orientação positiva e dois com orientação negativa) entre os pontos $(p, \vec{w})$ e $(p+1, \vec{w})$, enquanto o primeiro termo vem de dois pares de contribuições distintas com dois elos sobrepostos de orientações opostas ligando $\left(p, \vec{w}_{1}\right)$ a $\left(p+1, \vec{w}_{1}\right)$ e $\left(p, \vec{w}_{2}\right)$ a $\left(p+1, \vec{w}_{2}\right)$, respectivamente.

Para $M_{0}^{(4)}$, nós obtemos a estrutura do produto

$$
M_{0}^{(4)}=\frac{1}{2} M_{0}^{(0)} \circ M_{0}^{(0)}
$$

onde $\circ$ significa soma sobre $\vec{w}_{1}$ e $\vec{w}_{2}$ sem restrições; e notamos que os coeficientes do primeiro termo da eq. (B.32) e da eq. (B.33) são iguais.

Agora, fazendo $\Lambda=M^{-1}$ e $\Lambda_{0}=M_{0}^{-1}$ e notando que $K^{n=0,1,2,3}=0$, quando calculamos $K^{(4)}$ nós temos, para $x_{1}^{0}=x_{2}^{0}<x_{3}^{0}=x_{4}^{0}$,

$$
\begin{aligned}
K^{(4)} & \left(x_{1}, x_{2}, x_{3}, x_{4}\right)= \\
& =\left(\Lambda_{0}^{(0)} M_{0}^{(4)} \Lambda_{0}^{(0)}-\Lambda^{(0)} M^{(4)} \Lambda^{(0)}\right)\left(x_{1}, x_{2}, x_{3}, x_{4}\right) \\
& =\frac{1}{2}\left(\Lambda_{0}^{(0)} M_{0}^{(0)} \odot M_{0}^{(0)} \Lambda_{0}^{(0)}-\Lambda^{(0)} M^{(0)} \odot \Gamma M^{(0)} \Lambda^{(0)}\right)\left(x_{1}, x_{2}, x_{3}, x_{4}\right) \\
& =\frac{1}{2}(I-\Gamma) \sum_{\vec{w}} \delta\left(x_{1},(p, \vec{w})\right) \delta\left(x_{3},(p+1, \vec{w})\right) \delta\left(x_{2},(p, \vec{w})\right) \delta\left(x_{4},(p+1, \vec{w})\right),
\end{aligned}
$$

que fornece zero para distância temporal maior que uma unidade, i.e. $\left|x_{1}^{0}-x_{3}^{0}\right|>1$.

A quinta derivada de $K$ também é zero devido à ausência de balanceamento dos campos fermiônicos $\bar{\psi}$ e $\psi$ nas médias.

No caso de um sabor para $D_{0}$ obtemos

$$
D_{0}^{(4)}=\frac{1}{2} D_{0}^{(0)} \circ D_{0}^{(0)}
$$

Neste caso, a análoga da quarta derivada de $M$, dada pela equação (B.30), é, para $x_{1}^{0}=x_{2}^{0}<$ $x_{3}^{0}=x_{4}^{0}$,

$$
\begin{aligned}
& \langle F ; H\rangle_{T}^{(4)}\left(x_{1}, x_{2}, x_{3}, x_{4}\right)= \\
& \frac{1}{2} \sum_{\vec{w}_{1}, \vec{w}_{2} \mid \vec{w}_{1} \neq \vec{w}_{2}}\left\langle F\left(x_{1}, x_{2}\right) \Pi\left(p, \vec{w}_{1}\right) \Pi\left(p, \vec{w}_{2}\right)\right\rangle^{(0)}\left\langle\mu\left(p+1, \vec{w}_{1}\right) \mu\left(p+1, \vec{w}_{2}\right) H\left(x_{3}, x_{4}\right)\right\rangle^{(0)} \\
& +\frac{3}{4} \sum_{\vec{w}}\left\langle F\left(x_{1}, x_{2}\right) \Pi(p, \vec{w}) \Pi(p, \vec{w})\right\rangle^{(0)}\left\langle\mu(p+1, \vec{w}) \mu(p+1, \vec{w}) H\left(x_{3}, x_{4}\right)\right\rangle^{(0)}
\end{aligned}
$$


Fixando os campos externos, usando o fator $h$ da eq. (3.11), de tal forma que nós obtemos para a equação (B.35) a "estrutura do produto" nós obtemos para $K$ neste caso

$$
K^{(4)}\left(x_{1}, x_{2}, x_{3}, x_{4}\right)=\left(\Lambda_{0}^{(0)} D_{0}^{(4)} \Lambda_{0}^{(0)}-\Lambda^{(0)} D^{(4)} \Lambda^{(0)}\right)\left(x_{1}, x_{2}, x_{3}, x_{4}\right)=0 .
$$

que é zero para todas distâncias temporais, i.e. $\left|x_{3}^{0}-x_{1}^{0}\right|>1$.

O decaimento no espaço, i.e. quando a separação entre os hiperplanos se dá na direção espacial é obtido da mesma maneira. Usando a fórmula integral de Cauchy, conforme o Teorema 5, obtemos para o decaimento de $K$ os seguintes resultados:

No caso de um sabor

$$
\left|K\left(x_{1}, x_{2}, x_{3}, x_{4}\right)\right| \leq \kappa^{6\left|x_{3}^{0}-x_{1}^{0}\right|+\left|\vec{x}_{1}+\vec{x}_{2}-\vec{x}_{3}-\vec{x}_{4}\right|_{1}+\left|\vec{x}_{2}-\vec{x}_{1}\right|_{1}+\left|\vec{x}_{4}-\vec{x}_{3}\right|_{1}}
$$

No caso de dois sabores

$$
\left|K\left(x_{1}, x_{2}, x_{3}, x_{4}\right)\right| \leq\left\{\begin{aligned}
\kappa^{4\left|x_{3}^{0}-x_{1}^{0}\right|+\left|\vec{x}_{1}+\vec{x}_{2}-\vec{x}_{3}-\vec{x}_{4}\right|_{1}+\left|\vec{x}_{2}-\vec{x}_{1}\right|_{1}+\left|\vec{x}_{4}-\vec{x}_{3}\right|_{1}} & \left|x_{3}^{0}-x_{1}^{0}\right| \leq 1 \\
\kappa^{4} \kappa^{6\left(\left|x_{3}^{0}-x_{1}^{0}\right|-1\right)+\left|\vec{x}_{1}+\vec{x}_{2}-\vec{x}_{3}-\vec{x}_{4}\right|_{1}+\left|\vec{x}_{2}-\vec{x}_{1}\right|_{1}+\left|\vec{x}_{4}-\vec{x}_{3}\right|_{1}} & \left|x_{3}^{0}-x_{1}^{0}\right|>1
\end{aligned}\right.
$$

onde usamos a norma $|\vec{x}|_{1} \equiv\left|x^{1}\right|+\left|x^{2}\right|$. Este decaimento desempenha um papel fundamental na abordagem rigorosa para a determinação do estado ligado [33].

Nós agora explicamos a razão por trás da nossa restrição ao setor de paridade $(+1)$, ou seja, a escolha do estado $\mathbb{P}_{+}\left[\Pi\left(1 / 2, \vec{x}_{1}\right) \bar{\phi}\left(1 / 2, \vec{x}_{2}\right)\right]$. Nós desejamos um decaimento no tempo mais rápido que o decaimento de um méson-bárion que é $\kappa^{5\left|x_{3}^{0}-x_{1}^{0}\right|}$. Para obtermos o decaimento $\kappa^{7\left|x_{3}^{0}-x_{1}^{0}\right|}$ precisamos mostrar que $\partial_{0}^{n} \mathcal{K}=0(n=0,1,2,3)$. Os casos $n=0,1,2$ são fáceis já que podemos usar integrais sobre um único campo de calibre (veja a eq. (A.1)) e ausência de balanceamento nos campos fermiônicos que aparecem nas médias, para mostrarmos, respectivamente, $\partial_{0}^{1} \mathcal{K}=0$ e $\partial_{0}^{n} \mathcal{K}=0(n=0,2)$. Para provarmos que $\partial_{0}^{3} \mathcal{K}=0$ precisamos mostrar primeiramente que $\partial_{0}^{3} \mathcal{D}=0$. Nós daremos a seguir um breve cálculo para mostrarmos que $\partial_{0}^{3} \mathcal{D}=0$. Temos

$$
\partial_{0}^{3} \mathcal{D}\left(x_{1}, x_{2}, x_{3}, x_{4}\right)=6 \sum_{\vec{w}}\left\langle\mu\left(x_{1}\right) \phi\left(x_{2}\right) \bar{\phi}(p, \vec{w})\right\rangle^{(0)}\left\langle\phi(p, \vec{w}) P_{+}\left[\Pi\left(x_{3}\right) \bar{\phi}\left(x_{4}\right)\right]\right\rangle .
$$

Considerando o segundo termo no lado direito da eq. (B.38) e somando sobre translações de $\vec{x}_{3}$ e $\vec{x}_{4}$ para colocarmos o sistema com momento espacial zero com $\vec{w}$ fixado, i.e.

$$
F\left(x_{3}, x_{4}\right)=\int\left\langle\phi(p, \vec{w}) \mathbb{P}_{+}\left[\Pi\left(x_{3}+\vec{z}\right) \bar{\phi}\left(x_{4}+\vec{z}\right)\right]\right\rangle^{(0)} d \vec{z}
$$


Usando a simetria de paridade, seguida pela mudança de variáveis $\vec{z}^{\prime}=\vec{z}+2 \vec{w}$ obtemos $-F\left(x_{3}, x_{4}\right)$ de onde concluímos que $F\left(x_{3}, x_{4}\right)=0$ e daí $\partial_{0}^{3} \mathcal{D}=0$. Sem esta modificação teríamos $\partial_{0}^{3} \mathcal{K} \neq 0$ e usando estimativas de Cauchy, como fizemos no Teorema 3.2 para as derivadas de $\mathcal{K}$ teríamos apenas o decaimento $\kappa^{3\left|x_{3}^{0}-x_{1}^{0}\right|}$. 


\section{Apêndice C}

\section{Equação de Bethe-Salpeter em coordenadas relativas}

Neste apêndice, obtemos a equação de Bethe-Salpeter em coordenadas relativas a partir da eq. (3.13) para méson-méson e a similar para méson-bárion. As coordenadas relativas na rede são definidas como

$$
\xi=x_{2}-x_{1} \quad \eta=x_{4}-x_{3} \quad \text { e } \quad \tau=x_{3}-x_{2}
$$

com variáveis conjugadas no espaço de momentos dadas por $p, q$ e $k$, respectivamente. Esquematicamente, temos

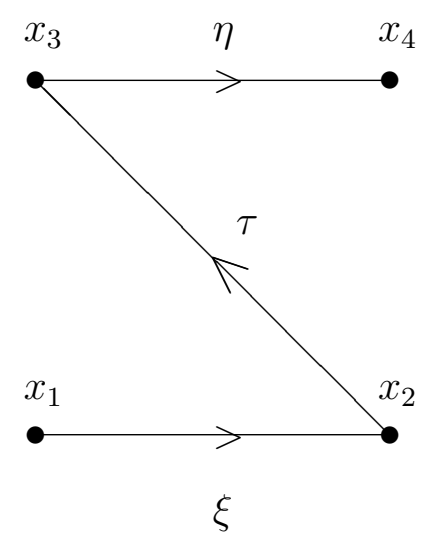

A equação com, $x_{1}^{0}=x_{2}^{0}$ e $x_{3}^{0}=x_{4}^{0}$ (i.e. $\left.\xi^{0}=0=\eta^{0}\right)$, é

$$
\begin{aligned}
& D\left(x_{1}, x_{2}, x_{3}, x_{4}\right)=D_{0}\left(x_{1}, x_{2}, x_{3}, x_{4}\right) \\
& +\int d y_{1} d y_{2} d y_{3} d y_{4} \delta\left(y_{1}^{0}-y_{2}^{0}\right) \delta\left(y_{3}^{0}-y_{4}^{0}\right) D\left(x_{1}, x_{2}, y_{1}, y_{2}\right) K\left(y_{1}, y_{2}, y_{3}, y_{4}\right) D_{0}\left(y_{3}, y_{4}, x_{3}, x_{4}\right)
\end{aligned}
$$


O núcleo de $D$ em coordenadas relativas é definido por

$$
\bar{D}(\xi, \eta, \tau)=D\left(0, x_{2}-x_{1}=\xi, x_{3}-x_{1}=\xi+\tau, x_{4}-x_{1}=\xi+\eta+\tau\right)
$$

e, de forma similar, definimos $D_{0}$ e, consequentemente, $K$. No caso de méson-méson, como $\mu, \Pi \in \mathcal{H}_{e}$ o núcleo de B-S, $K\left(x_{1}, x_{2}, x_{3}, x_{4}\right)$, é invariante pelas transformações de coordenadas

$$
\left(x_{1}, x_{2}, x_{3}, x_{4}\right) \rightarrow\left(x_{2}, x_{1}, x_{3}, x_{4}\right) ;\left(x_{1}, x_{2}, x_{3}, x_{4}\right) \rightarrow\left(x_{2}, x_{1}, x_{4}, x_{3}\right) .
$$

Estas relações implicam nas seguintes simetrias para $\bar{K}(\xi, \eta, \tau)$

$$
\bar{K}(\xi, \eta, \tau)=\bar{K}(-\xi, \eta, \tau+\xi) ; \bar{K}(\xi, \eta, \tau)=\bar{K}(\xi,-\eta, \tau+\eta)
$$

Nós agora introduzimos as variáveis $\xi^{\prime}, \eta^{\prime}, \tau^{\prime}$ e $\tau^{\prime \prime}$ onde

$$
\xi^{\prime}=y_{2}-y_{1}, \eta^{\prime}=y_{4}-y_{3}, \tau^{\prime}=y_{1}-x_{2} \text { e } \tau^{\prime \prime}=x_{3}-y_{4} .
$$

Então,

$$
y_{1}=\tau^{\prime}+x_{2}, y_{2}=\xi^{\prime}+y_{1}=\xi^{\prime}+\tau^{\prime}+x_{2}, y_{4}=x_{3}-\tau^{\prime \prime} \text { e } y_{3}=y_{4}-\eta^{\prime}=x_{3}-\tau^{\prime \prime}-\eta^{\prime} .
$$

Desta forma, obtemos

$$
D\left(x_{1}, x_{2}, y_{1}, y_{2}\right)=\bar{D}\left(\xi, \xi^{\prime}, \tau^{\prime}\right) ; D_{0}\left(y_{3}, y_{4}, x_{3}, x_{4}\right)=\bar{D}_{0}\left(\eta^{\prime}, \eta, \tau^{\prime \prime}\right)
$$

e, então,

$$
\begin{aligned}
K\left(y_{1}, y_{2}, y_{3}, y_{4}\right) & =\bar{K}\left(\xi^{\prime}, \eta^{\prime}, \tau-\tau^{\prime}-\tau^{\prime \prime}-\xi^{\prime}-\eta^{\prime}\right) \\
& =\bar{K}\left(-\xi^{\prime},-\eta^{\prime}, \tau-\tau^{\prime}-\tau^{\prime \prime}\right)
\end{aligned}
$$

onde, para obtermos a primeira igualdade em (C.6), usamos (C.5), i.e.

$$
y_{3}-y_{2}=\left(x_{3}-\tau^{\prime \prime}-\eta^{\prime}\right)-\left(\xi^{\prime}+\tau^{\prime}+x_{2}\right)=\tau-\tau^{\prime}-\tau^{\prime \prime}-\xi^{\prime}-\eta^{\prime}
$$

e para obtermos a segunda usamos a simetria (C.4). A equação de B-S em coordenadas relativas fica, então, dada por

$$
\bar{D}(\vec{\xi}, \vec{\eta}, \tau)=\bar{D}_{0}(\vec{\xi}, \vec{\eta}, \tau)+\int d \vec{\xi}^{\prime} d \vec{\eta}^{\prime} d \tau^{\prime} d \tau^{\prime \prime} \bar{D}\left(\vec{\xi}, \vec{\xi}^{\prime}, \tau^{\prime}\right) \bar{K}\left(-\vec{\xi}^{\prime},-\vec{\eta}^{\prime}, \tau-\tau^{\prime}-\tau^{\prime \prime}\right) \bar{D}_{0}\left(\vec{\eta}^{\prime}, \vec{\eta}, \tau^{\prime \prime}\right)
$$


Tomando a transformada de Fourier na variável $\tau$, obtemos finalmente

$$
\bar{D}(\vec{\xi}, \vec{\eta}, k)=\bar{D}_{0}(\vec{\xi}, \vec{\eta}, k)+\int \bar{D}\left(\vec{\xi}, \vec{\xi}^{\prime}, k\right) \bar{K}\left(-\vec{\xi}^{\prime},-\vec{\eta}^{\prime}, k\right) \bar{D}_{0}\left(\vec{\eta}^{\prime}, \vec{\eta}, k\right)
$$

Quando $k=\left(k^{0}, \overrightarrow{0}\right)$, a eq. (C.9) pode ser reescrita da seguinte forma

$$
\bar{D}(\vec{\xi}, \vec{\eta}, k)=\bar{D}_{0}(\vec{\xi}, \vec{\eta}, k)+\int \bar{D}\left(\vec{\xi}, \vec{\xi}^{\prime}, k\right) \bar{K}\left(\vec{\xi}^{\prime}, \vec{\eta}^{\prime}, k\right) \bar{D}_{0}\left(\vec{\eta}^{\prime}, \vec{\eta}, k\right),
$$

onde usamos as simetrias dadas pela eq. (C.4), e lembrando que estamos somando sobre $\vec{\tau}$.

Nós notamos que, no caso de méson-bárion, não temos as simetrias dadas pela eq. (C.4). Neste caso, o campo $\mu(\Pi)$ em $x_{1}\left(x_{3}\right)$ é distinto do campo $\phi(\bar{\phi})$ em $x_{2}\left(x_{4}\right)$. Considerando $K$ e tomando a transformada de Fourier na variável $\tau$, temos

$$
\begin{aligned}
& \int d \tau e^{i \tau^{0} k^{0}} \bar{K}\left(\vec{\xi}^{\prime}, \vec{\eta}^{\prime}, \tau-\tau-\tau^{\prime \prime}-\vec{\xi}^{\prime}-\vec{\eta}^{\prime}\right) \\
& =\int d \tau e^{i \tau^{0} k^{0}} K\left(0, \vec{\xi}^{\prime}, \tau-\tau^{\prime}-\tau^{\prime \prime}-\vec{\eta}^{\prime}, \tau-\tau^{\prime}-\tau^{\prime \prime}\right) \\
& =\int d \tau e^{i \tau^{0} k^{0}} K\left(0, \vec{\xi}^{\prime},\left(t+\vec{\xi}^{\prime}+\vec{\eta}^{\prime}\right)-\tau^{\prime}-\tau^{\prime \prime}-\vec{\eta}^{\prime},\left(t+\vec{\xi}^{\prime}+\vec{\eta}^{\prime}\right)-\tau^{\prime}-\tau^{\prime \prime}\right) \\
& =\int d \tau e^{i \tau^{0} k^{0}} K\left(0, \vec{\xi}^{\prime}, t-\tau^{\prime}-\tau^{\prime \prime}+\vec{\xi}^{\prime}, t-\tau^{\prime}-\tau^{\prime \prime}+\vec{\xi}^{\prime}+\vec{\eta}^{\prime}\right) \\
& =\int d t e^{i t^{0} k^{0}} \bar{K}\left(\vec{\xi}^{\prime}, \vec{\eta}^{\prime}, t-\tau^{\prime}-\tau^{\prime \prime}\right)
\end{aligned}
$$

onde, para obtermos a última igualdade, fizemos a mudança de variáveis $\tau \rightarrow t+\vec{\xi}^{\prime}+\vec{\eta}^{\prime}$. Pela análise anterior, obtemos a eq. (C.9) para o méson-bárion.

A eq. (C.9) será usada na análise da existência dos estados ligados nos Capítulos 3 e 4. Nesta tese, com um abuso de notação, omitimos a barra em cima de um dado operador sempre que o escrevermos em coordenadas relativas. 


\section{Referências Bibliográficas}

[1] T. Balaban, Commun. Math. Phys. 122, 175 (1989); 122, 355 (1989); 119, 243 (1988); 116, 1 (1988).

[2] T. Banks, S. Raby, L. Susskind, J. Kogut, D.R.T. Jones, P.N. Scharbach and D.K. Sinclair, Phys. Rev. D 15, 1111 (1977).

[3] F.A. Berezin, The Method of Second Quantization (Academic Press, NY, 1966).

[4] J. Bricmont and J. Fröhlich, Nucl. Phys. B 251, 517 (1985); Commun. Math. Phys. 98, 553 (1985); Nucl. Phys. B 280, 385 (1987).

[5] M. Creutz, Quarks, Gluons and Lattices (Cambridge University Press, Cambridge, 1983).

[6] M. Creutz, Rev. Mod. Phys. 50, 561 (1978).

[7] M. Creutz, J. Math. Phys. 19, 2043 (1978).

[8] M. Creutz, Nucl. Phys. B (Proc. Suppl.) 94, 219 (2001).

[9] J. Dimock, J.-P. Eckmann, Commun. Math. Phys. 51, 41 (1976); Annals of Physics (NY) 103, 289 (1977). J. Dimock, J. Stat. Phys. 58, 1181 (1990).

[10] P.A. Faria da Veiga, M. O'Carroll, E. Pereira e R. Schor, Commun. Math. Phys. 220 (2001), 377 .

[11] P.A. Faria da Veiga, M. O'Carroll e R. Schor, Phys. Rev. D 67, 017501 (2003).

[12] P.A. Faria da Veiga, M. O'Carroll e R. Schor, Commun. Math. Phys. 245, 383 (2004).

[13] P.A. Faria da Veiga, M. O'Carroll e R. Schor, Phys. Rev. D 68, 037501 (2003).

[14] P.A. Faria da Veiga e M. O'Carroll e A. Francisco Neto, Phys. Rev. D 69, 097501 (2004), $4 \mathrm{pp}$. 
[15] P.A. Faria da Veiga e M. O'Carroll, Phys. Rev. D 71, 017503 (2005).

[16] P.A. Faria da Veiga e M. O'Carroll e A. Francisco Neto, Phys. Rev. D 72, 034507 (2005), 18 pp.

[17] H.R. Fiebig and H. Markum in International Review of Nuclear Physics, Hadronic Physics from Lattice QCD, A.M. Green ed. (World Scientific, Singapoure, 2003).

[18] J. Feldman, J. Magnen, V. Rivasseau, R. Sénéor, Commun. Math. Phys. 155, 325 (1993).

[19] A. Francisco Neto, P.A. Faria da Veiga e M. O'Carroll J. Math. Phys. 45, 628-641 (2004).

[20] A. Francisco Neto, Phys. Rev. D 70, 037502 (2004), 4pp.

[21] K. Fredenhagen, Commun. Math. Phys. 101, 579 (1984).

[22] J. Fröhlich e C. King, Nucl. Phys B 290, 157 (1987).

[23] J. Glimm e A. Jaffe, Quantum Physics: A Functional Integral Point of View (Springer Verlag, New York, 1986).

[24] E.P. Wigner, Group Theory (Academic Press, New York, 1959).

[25] M. Luscher, Commun. Math. Phys. 54, 283 (1977).

[26] R. Machleidt, Nucl. Phys. A 689, 11c (2001).

[27] R. Machleidt, K. Holinde, e Ch. Elster, Phys. Rep. 149, 1 (1987).

[28] I. Montvay e G. Münster, Quantum Fields on a Lattice (Cambridge University Press, Cambridge, 1997).

[29] I. Montvay, Rev. Mod. Phys. 59 (1987), 263.

[30] K. Osterwalder e E. Seiler, Ann. Phys. (N.Y.) 110, 440 (1978).

[31] R. Schor, Nucl. Phys. B 222, 71 (1983); B 231, 321 (1984).

[32] R. Schor, Commun. Math. Phys. 59, 213 (1978).

[33] R. Schor and M. O'Carroll, J. Stat. Phys. 99, 1265 (2000); 109, 279 (2002).

[34] D. Schreiber, Phys. Rev. D 48, 5393 (1983). 
[35] E. Seiler, Gauge Theories as a Problem of Constructive Quantum Field Theory and Statistical Mechanics, Lecture Notes in Physics 159, Springer, New York, 1982.

[36] B. Simon, Statistical Mechanics of Lattice Models (Princeton University Press, Princeton, 1994).

[37] B. Simon, Representations of Finite and Compact Groups (American Mathematical Society, Providence, 1996).

[38] T. Spencer, Commun. Math. Phys. 44, 143 (1975).

[39] T. Spencer, F. Zirilli, Commun. Math. Phys. 49, 1 (1976).

[40] C. Stewart and R. Koniuk, Phys. Rev. D 57, 5581 (1998).

[41] K. Wilson in New Phenomena in Subnuclear Physics, Part A, edited by A. Zichichi, Plenum Press, New York, 1977. 